Supporting Information for:

\title{
Photochromic Radical Complexes that Show Heterolytic Bond Dissociation
}

Ryosuke Usui ${ }^{\dagger}$, Katsuya Yamamoto ${ }^{\ddagger}$,Hajime Okajima ${ }^{\ddagger}$,Katsuya Mutoh ${ }^{\ddagger}$, Akira Sakamoto ${ }^{\ddagger}$, Jiro Abe $e^{t, *}$ and Yoichi Kobayashit,*

†Department of Applied Chemistry, College of Life Sciences, Ritsumeikan University, 1-1-1 Nojihigashi, Kusatsu, Shiga 525-8577, Japan.

Department of Chemistry, School of Science and Engineering, Aoyama Gakuin University, 5-10-1 Fuchinobe, Chuoku, Sagamihara, Kanagawa 252-5258, Japan.

E-mail: ykobayas@fc.ritsumei.ac.jp jiro_abe@chem.aoyama.ac.jp

\section{CONTENTS}

1. ${ }^{1} \mathrm{H}$ NMR Spectra $\quad$ S2

2. HR-ESI-TOF-MS Spectra $\quad$ S5

3. HPLC Chromatograms $\quad$ S6

4. X-ray Crystallographic Analyses $\quad$ S8

5. Steady-State Absorption Spectra at Different Solvents $\quad$ S10

6. Details of the Nanosecond to Microsecond Transient Absorption Measurements $\quad$ S11

7. Nanosecond to Microsecond Transient Absorption Spectra at Different Solvents $\quad$ S13

$\begin{array}{ll}\text { 8. Cyclic Voltammetry Measurements } & \text { S17 }\end{array}$

9. Femtosecond to Nanosecond Transient Absorption Measurements $\quad$ S18

$\begin{array}{ll}\text { 10. Picosecond Time-Resolved IR spectra } & \text { S27 }\end{array}$

11. Estimation of the Relative Energy Levels of the CT States to the $S_{1}$ states $\quad$ S28

12. DFT calculations $\quad$ S29

$\begin{array}{ll}\text { 13. References } & \text { S111 }\end{array}$ 


\section{1. ${ }^{1} \mathrm{H}$ NMR Spectra}

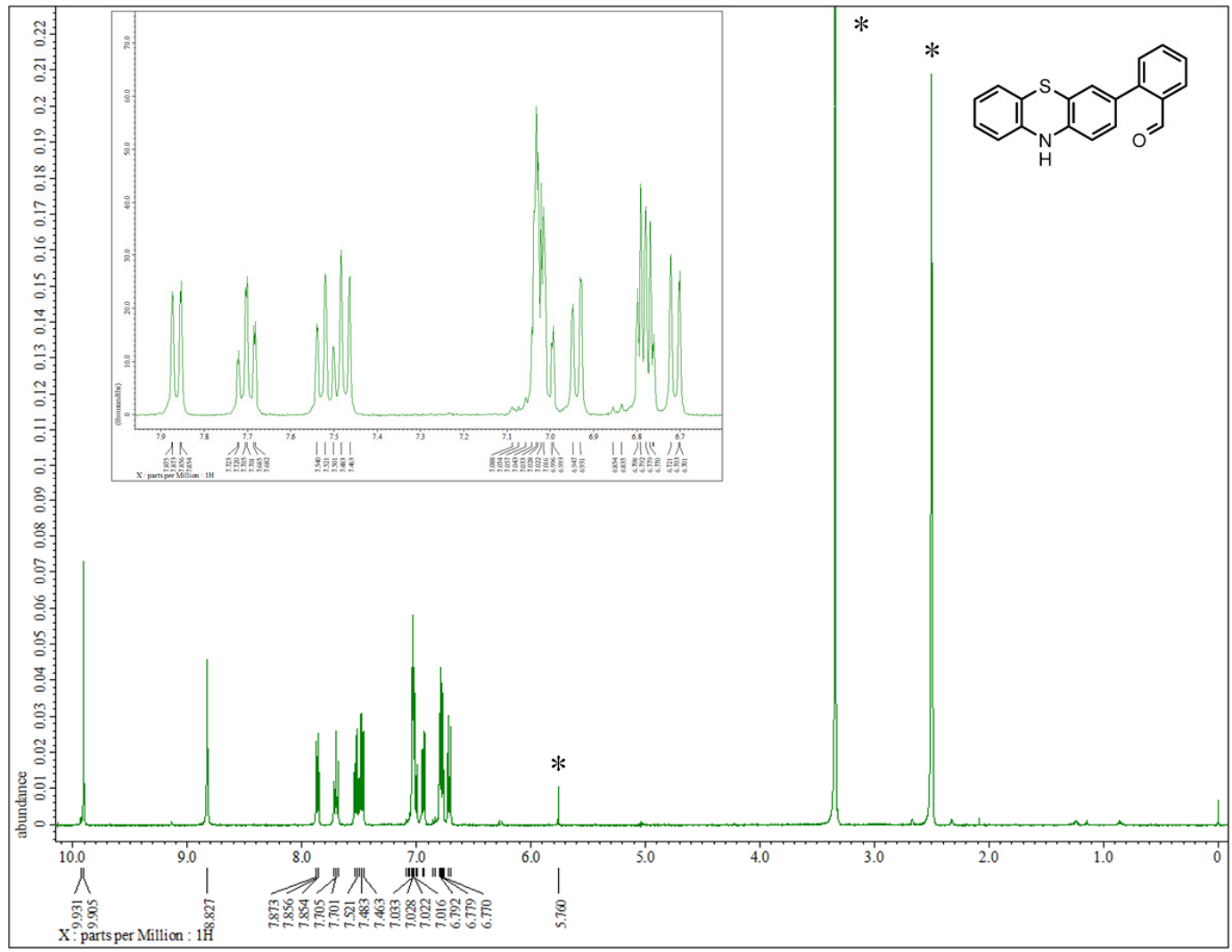

Figure S1. ${ }^{1} \mathrm{H}$ NMR spectrum of 4 in DMSO- $d 6$ (* solvent peaks).

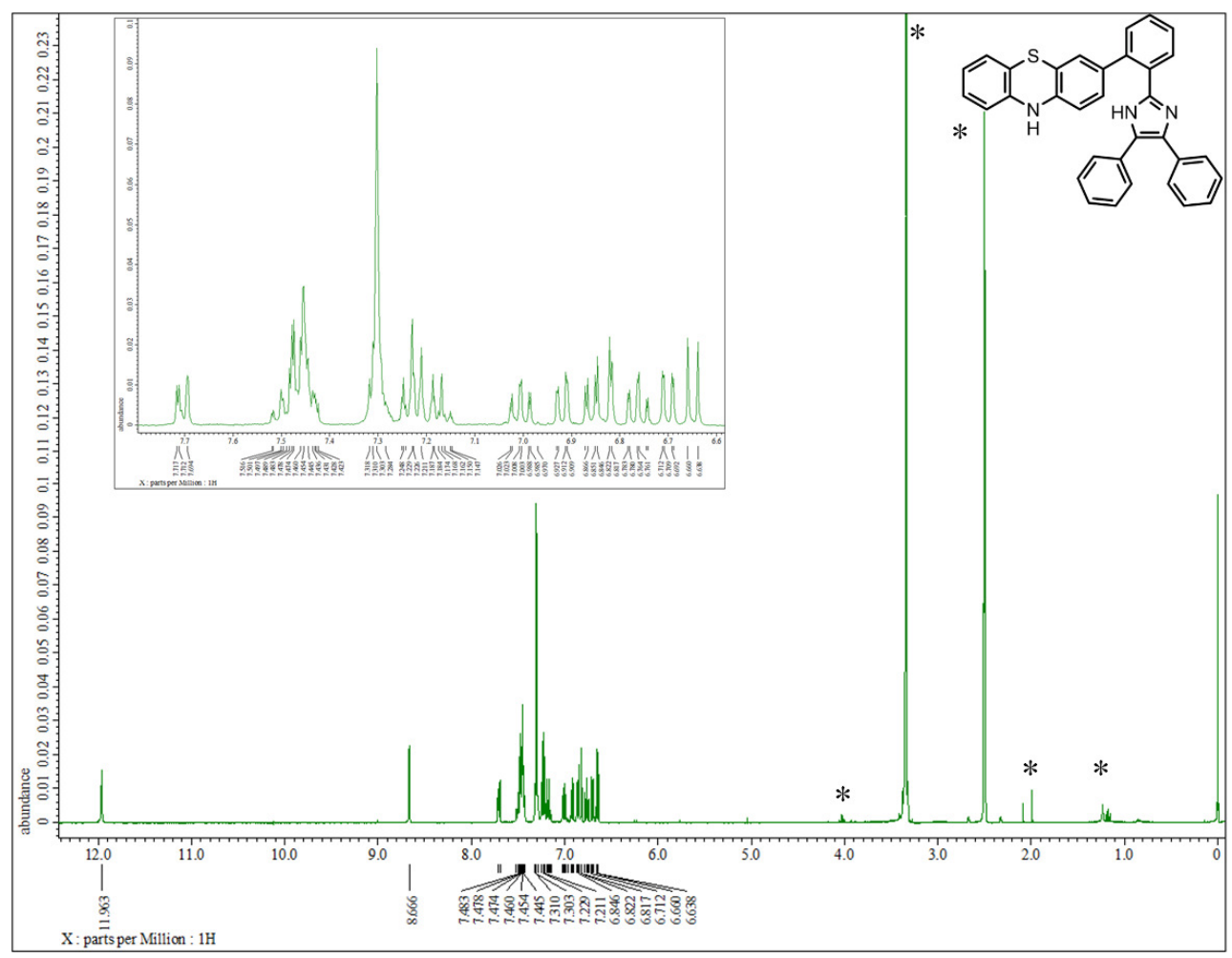

Figure S2. ${ }^{1} \mathrm{H}$ NMR spectrum of 5 in DMSO- $d 6$ (* solvent peaks). 


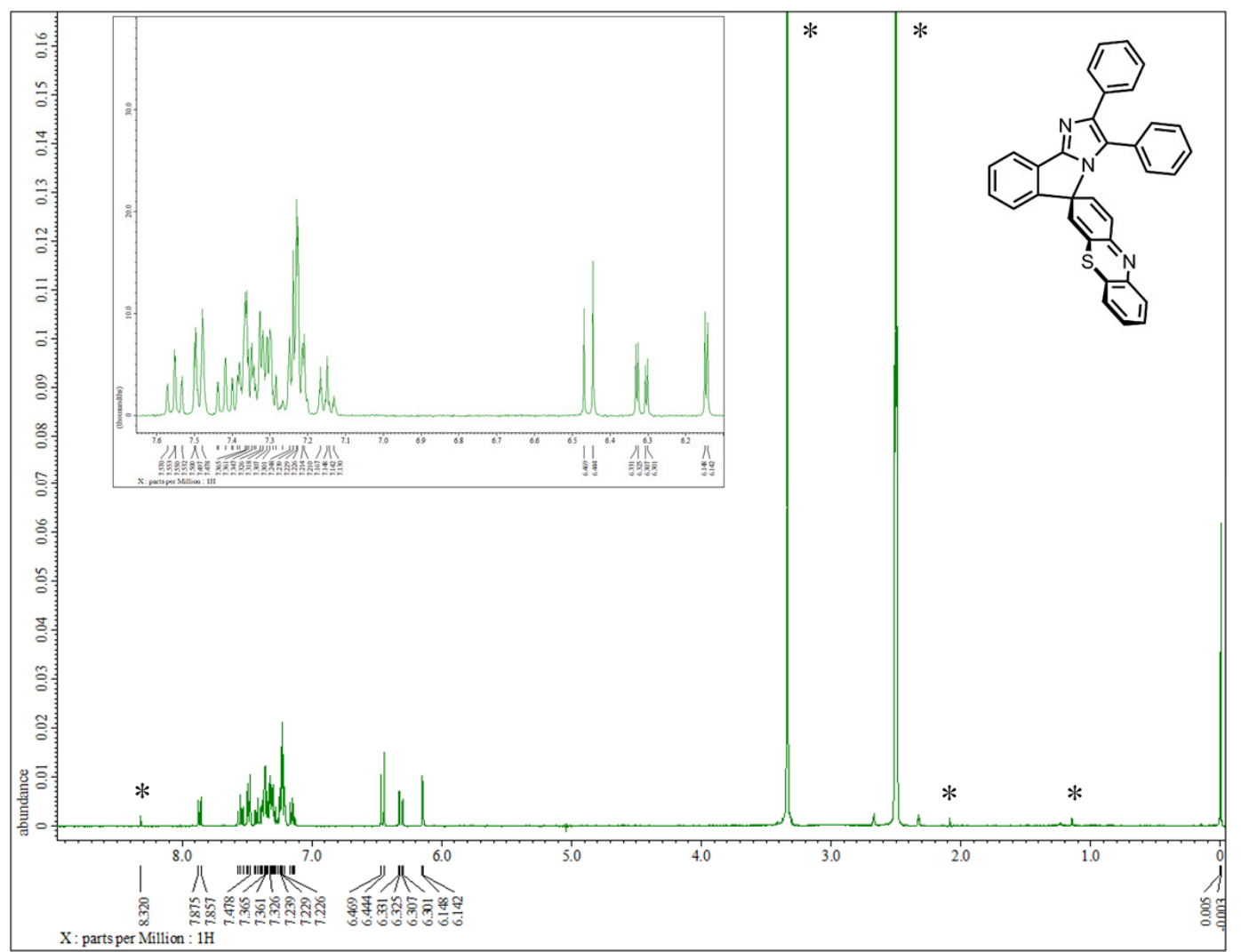

Figure S3. ${ }^{1} \mathrm{H}$ NMR spectrum of 1 in DMSO- $d 6$ (* solvent peaks).

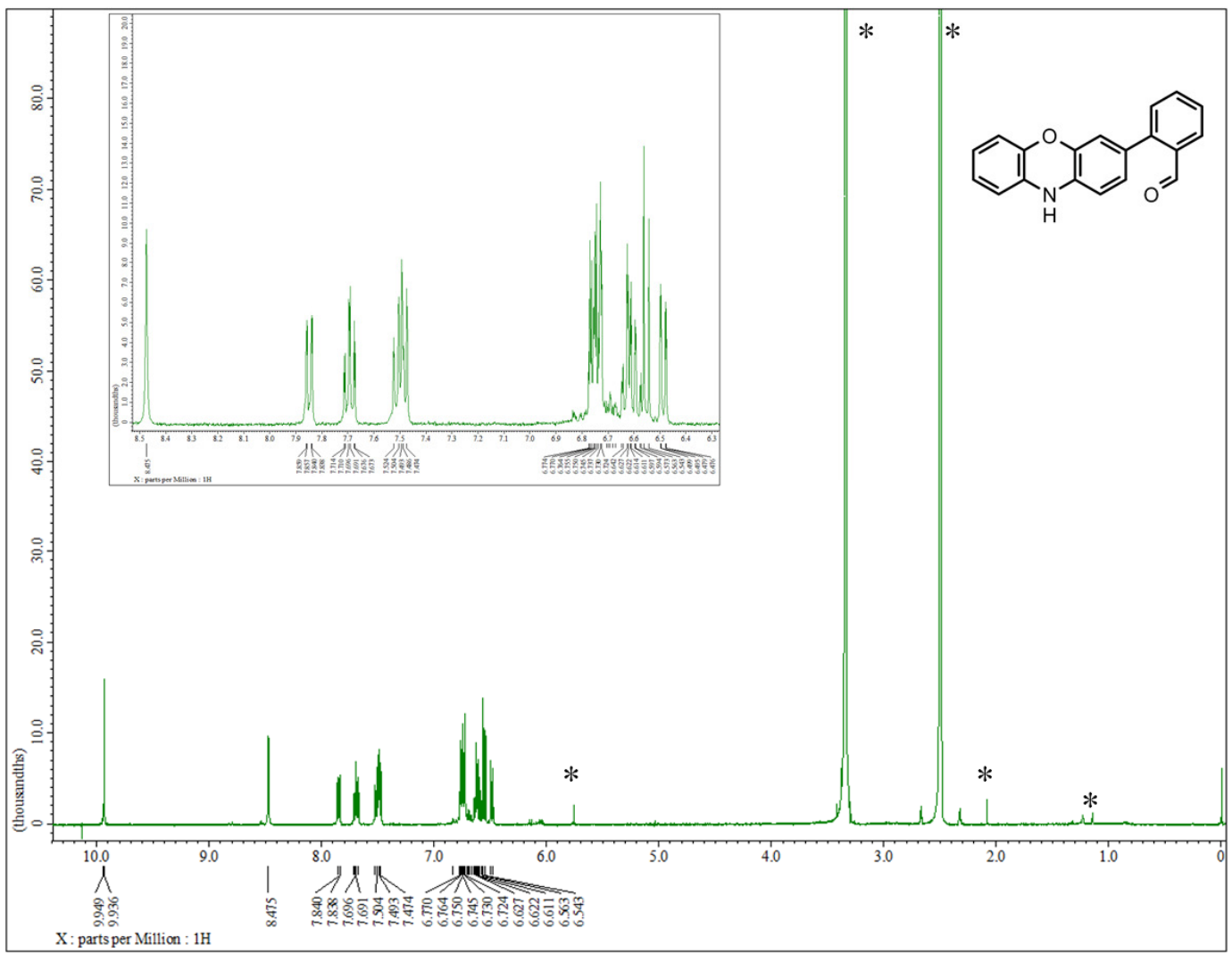

Figure S4. ${ }^{1} \mathrm{H}$ NMR spectrum of 7 in DMSO- $d 6$ (* solvent peaks). 


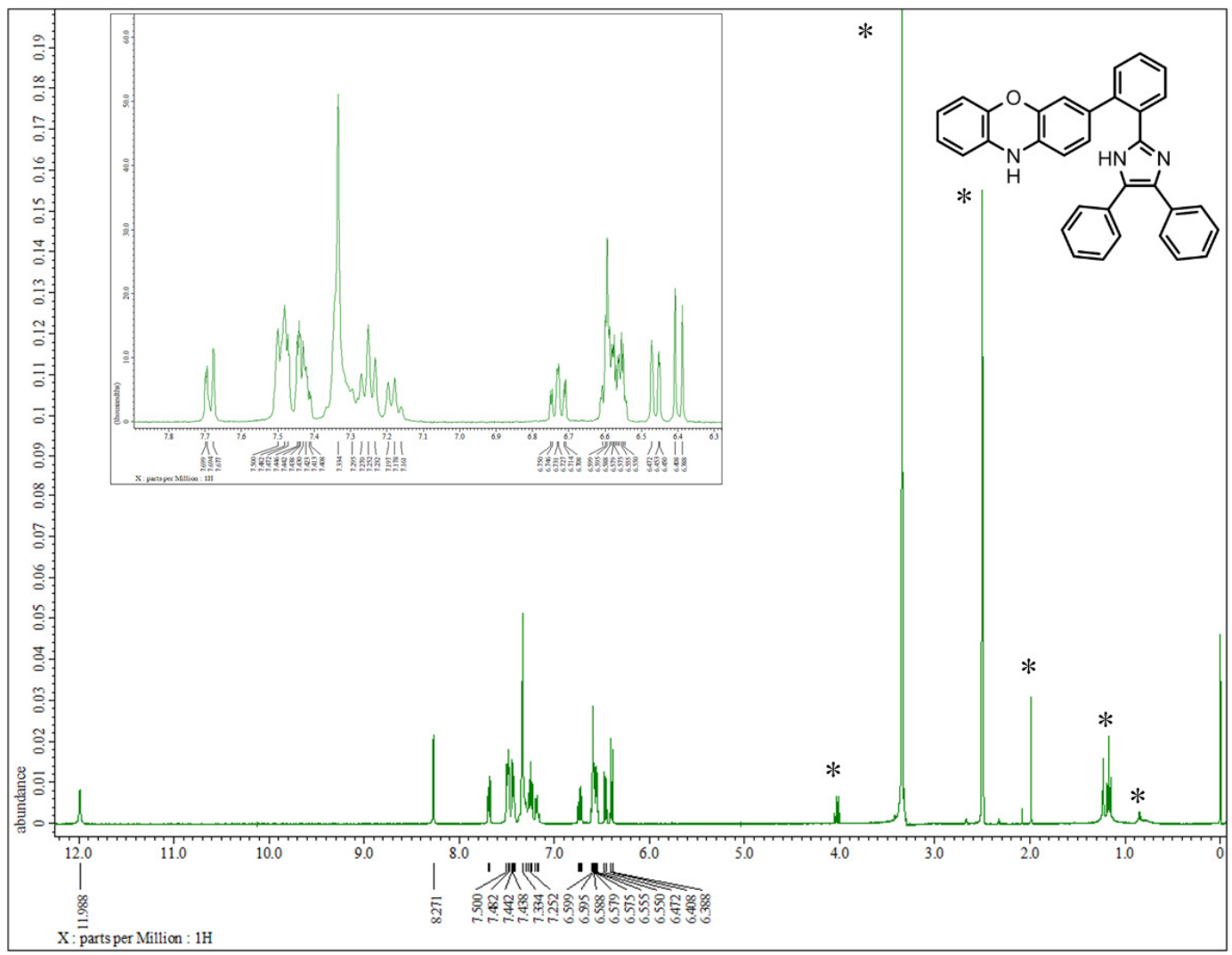

Figure S5. ${ }^{1} \mathrm{H}$ NMR spectrum of 8 in DMSO- $d 6$ (* solvent peaks).

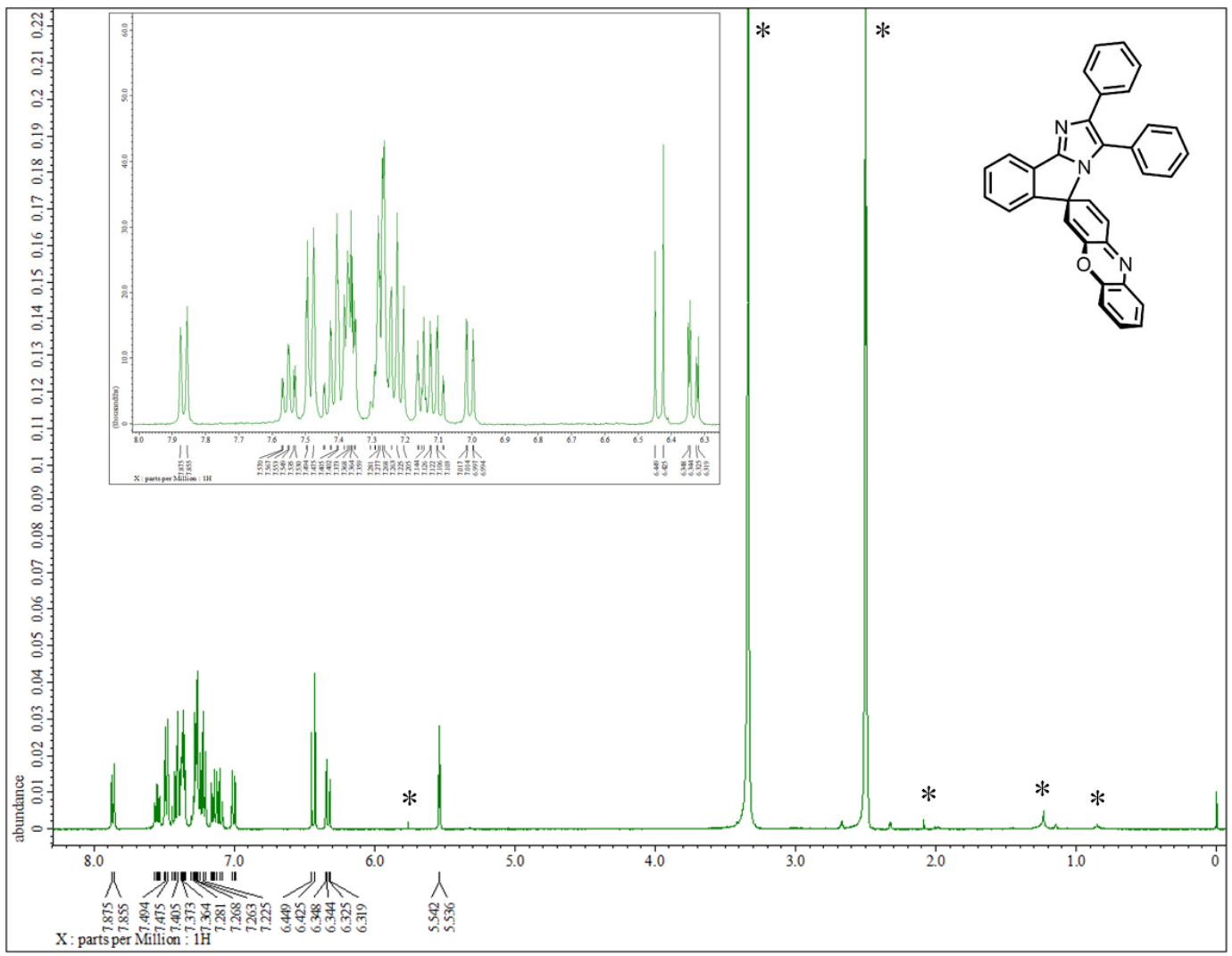

Figure S6. ${ }^{1} \mathrm{H}$ NMR spectrum of 2 in DMSO- $d 6$ (* solvent peaks). 


\section{HR-ESI-TOF-MS Spectra}

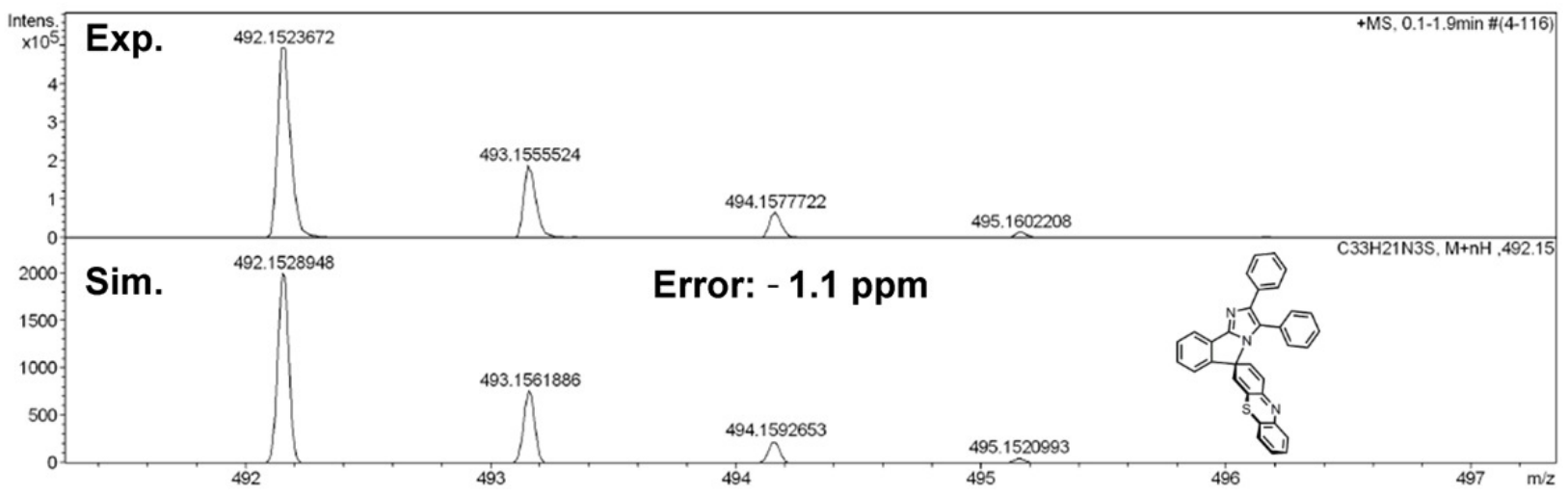

Figure S7. HR-ESI-TOF-MS of 1.

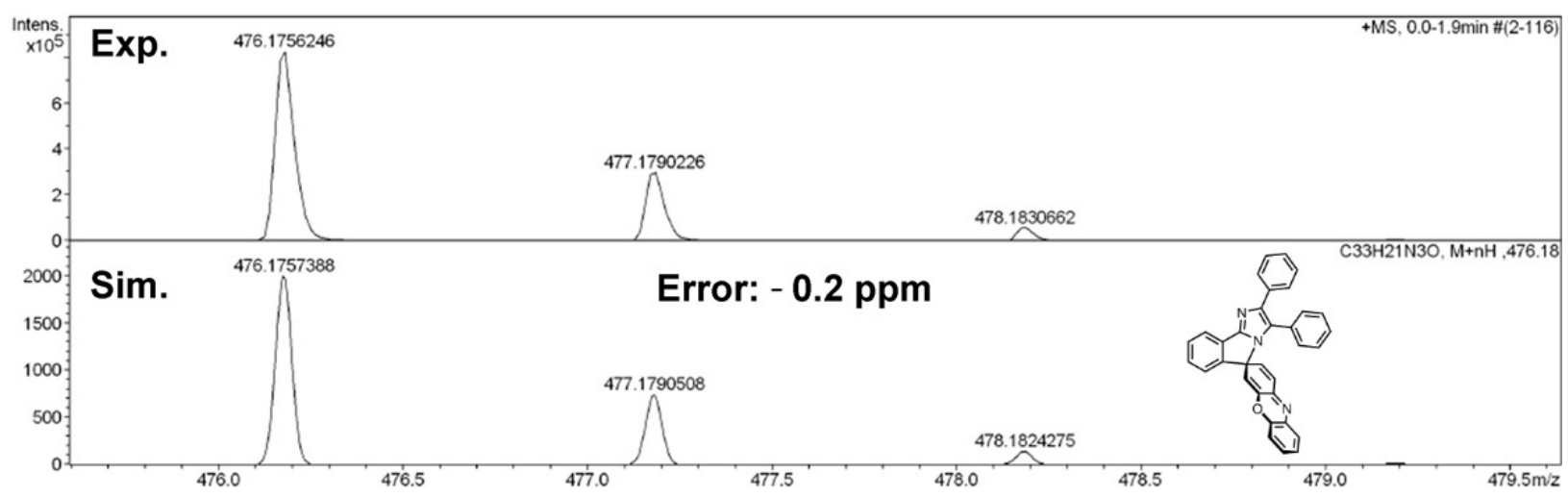

Figure S8. HR-ESI-TOF-MS of 2. 


\section{HPLC Chromatograms}

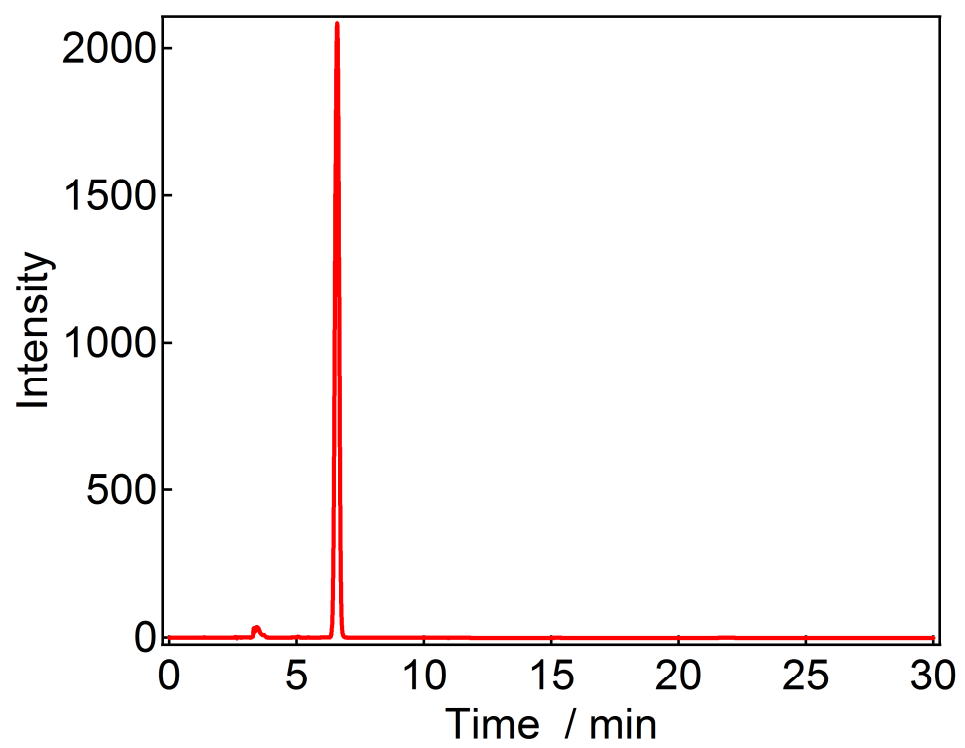

Figure S9. HPLC chromatogram of 1; 99\% purity. HPLC analysis was performed using a reverse phase analytical column (Mightysil RP18GP II, $25 \mathrm{~cm} \times 4.6 \mathrm{~mm}, 5 \mu \mathrm{m}$ particle) from Kanto Chemical Industries, equipped with a PDA detector; the mobile phase was $\mathrm{CH}_{3} \mathrm{CN} / \mathrm{H}_{2} \mathrm{O}=9 / 1$ with a flow rate of $1.0 \mathrm{~mL} / \mathrm{min}$ (detection wavelength; $254 \mathrm{~nm}$ ). It is noted that peaks below $5 \mathrm{~min}$ are due to the injection solvent.

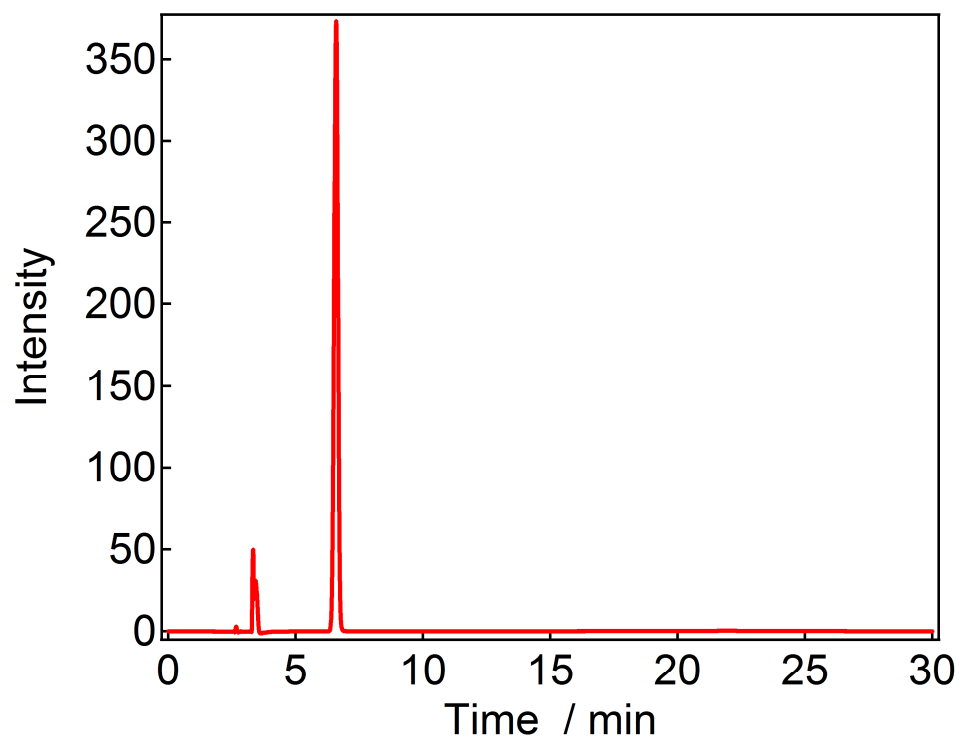

Figure S10 HPLC chromatogram of 1; 99\% purity. HPLC analysis was performed using a reverse phase analytical column (Mightysil RP18GP II, $25 \mathrm{~cm} \times 4.6 \mathrm{~mm}, 5 \mu \mathrm{m}$ particle) from Kanto Chemical Industries, equipped with a PDA detector; the mobile phase was $\mathrm{CH}_{3} \mathrm{CN} / \mathrm{H}_{2} \mathrm{O}=9 / 1$ with a flow rate of $1.0 \mathrm{~mL} / \mathrm{min}$ (detection wavelength; $420 \mathrm{~nm}$ ). It is noted that peaks below $5 \mathrm{~min}$ are due to the injection solvent. 


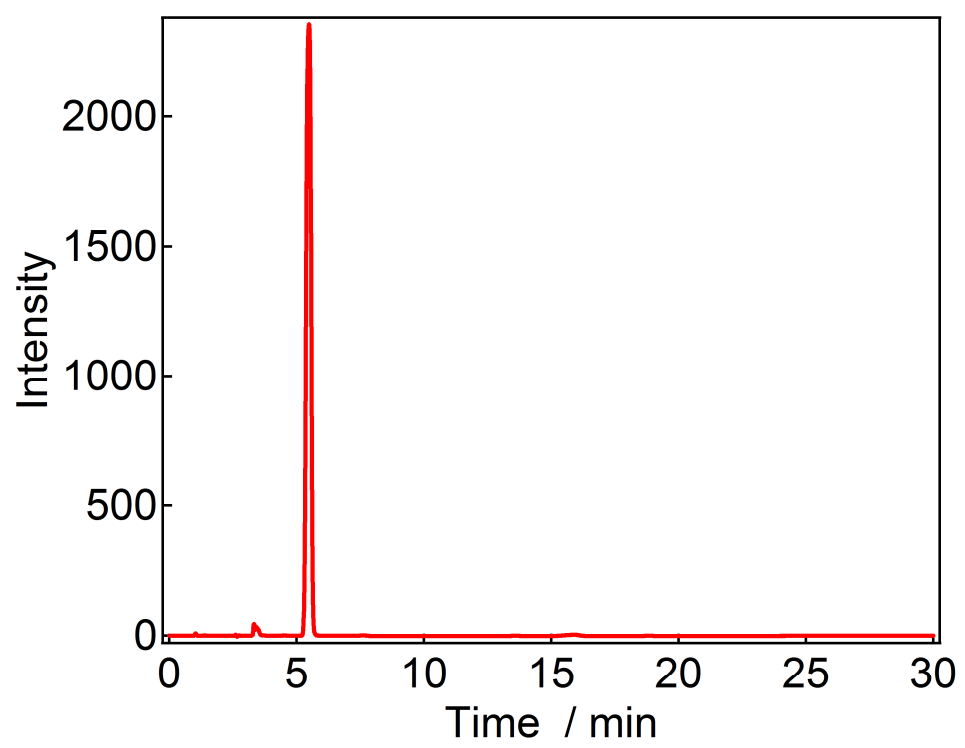

Figure S11. HPLC chromatogram of 2; 99\% purity. HPLC analysis was performed using a reverse phase analytical column (Mightysil RP18GP II, $25 \mathrm{~cm} \times 4.6 \mathrm{~mm}, 5 \mu \mathrm{m}$ particle) from Kanto Chemical Industries, equipped with a PDA detector; the mobile phase was $\mathrm{CH}_{3} \mathrm{CN} / \mathrm{H}_{2} \mathrm{O}=9 / 1$ with a flow rate of $1.0 \mathrm{~mL} / \mathrm{min}$ (detection wavelength; $254 \mathrm{~nm}$ ). It is noted that peaks below $5 \mathrm{~min}$ are due to the injection solvent.

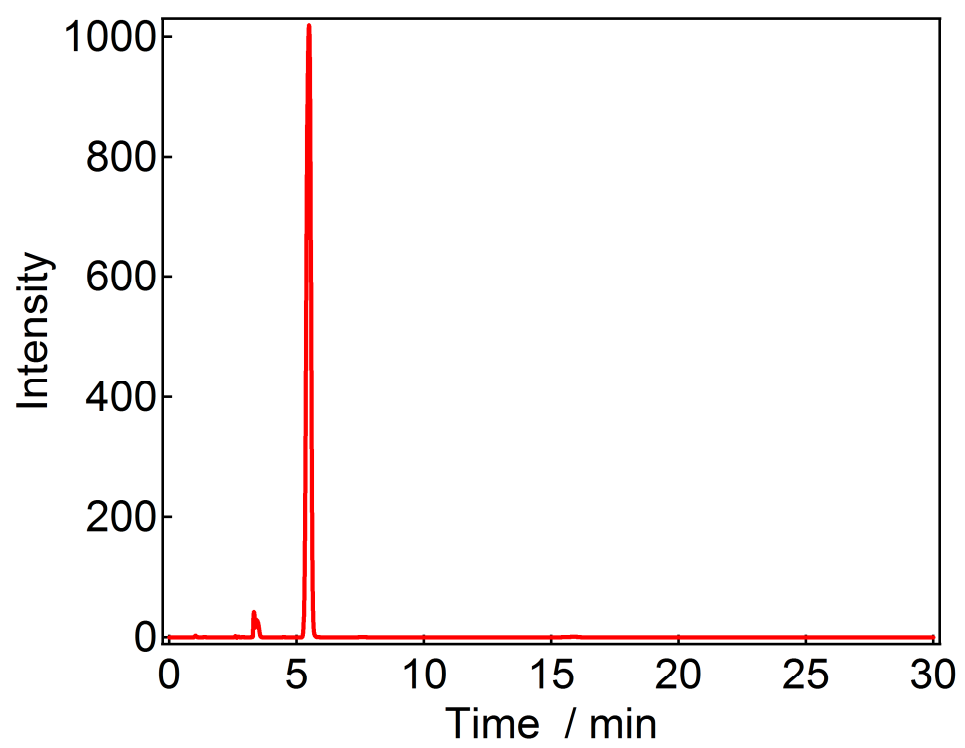

Figure S12. HPLC chromatogram of 2; 99\% purity. HPLC analysis was performed using a reverse phase analytical column (Mightysil RP18GP II, $25 \mathrm{~cm} \times 4.6 \mathrm{~mm}, 5 \mu \mathrm{m}$ particle) from Kanto Chemical Industries, equipped with a PDA detector; the mobile phase was $\mathrm{CH}_{3} \mathrm{CN} / \mathrm{H}_{2} \mathrm{O}=9 / 1$ with a flow rate of $1.0 \mathrm{~mL} / \mathrm{min}$ (detection wavelength; $380 \mathrm{~nm}$ ). It is noted that peaks below $5 \mathrm{~min}$ are due to the injection solvent. 


\section{X-ray Crystallographic Analyses}

Table S1. Crystallographic Parameters of 1

Empirical formula

Formula weight

Temperature

Wavelength

Crystal system

Space group

Unit cell dimensions

Volume

Z

Density (calculated)

Absorption coefficient

$\mathrm{F}(000)$

Theta range for data collection

Index ranges

Reflections collected

Independent reflections

Absorption correction

Refinement method

Data / restraints / parameters

Goodness-of-fit on $\mathrm{F}^{2}$

Final $\mathrm{R}$ indices $[\mathrm{I}>2 \operatorname{sigma}(\mathrm{I})]$

$\mathrm{R}$ indices (all data)

Largest diff. peak and hole
$\mathrm{C}_{33} \mathrm{H}_{21} \mathrm{~N}_{3} \mathrm{~S}$

491.59

100.01(10) K

$0.71073 \AA$

monoclinic

I $2 / \mathrm{a}$

$\mathrm{a}=18.7383(3) \AA \quad \alpha=90^{\circ}$

$\mathrm{b}=11.5764(3) \AA \quad \beta=93.211(2)^{\circ}$

$\mathrm{c}=22.1767(5) \AA \quad \gamma=90^{\circ}$

4803.06(18) $\AA^{3}$

8

$1.360 \mathrm{~g} / \mathrm{m}^{3}$

$0.164 \mathrm{~mm}^{-1}$

2048.0

3.678 to $61.198^{\circ}$

$-26<=\mathrm{h}<=26,-15<=\mathrm{k}<=16,-29<=1<=30$

24678

$6980[\mathrm{R}(\mathrm{int})=0.0226]$

Empirical

Full-matrix least-squares on $\mathrm{F}^{2}$

$6980 / 0 / 335$

1.063

$\mathrm{R} 1=0.0547, \mathrm{wR} 2=0.1433$

$\mathrm{R} 1=0.0698, \mathrm{wR} 2=0.1545$

0.73 and $-0.57 \mathrm{e}^{-3}$

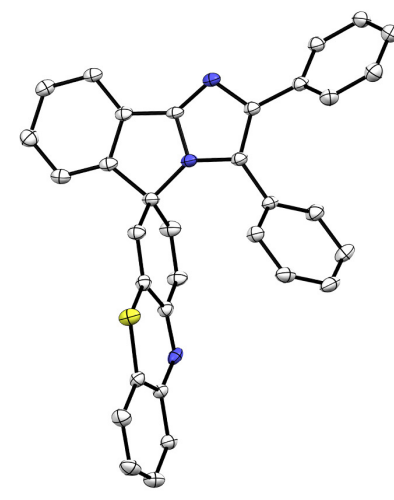

Figure S13. ORTEP representation of the molecular structure of 1 with thermal ellipsoids (50\% probability), where sulfur and nitrogen atoms are highlighted in yellow and blue. 
Empirical formula

Formula weight

Temperature

Wavelength

Crystal system

Space group

Unit cell dimensions

Volume

Z

Density (calculated)

Absorption coefficient

$\mathrm{F}(000)$

Theta range for data collection

Index ranges

Reflections collected

Independent reflections

Absorption correction

Refinement method

Data / restraints / parameters

Goodness-of-fit on $\mathrm{F}^{2}$

Final R indices [I $>2 \operatorname{sigma}(\mathrm{I})]$

$\mathrm{R}$ indices (all data)

Largest diff. peak and hole
$\mathrm{C}_{33} \mathrm{H}_{21} \mathrm{~N}_{3} \mathrm{O}$

475.53

100.00(10) K

$0.71073 \AA$

triclinic

P-1

$\mathrm{a}=6.8256(4) \AA \quad \alpha=92.607(5)^{\circ}$

$\mathrm{b}=12.4886(8) \AA \quad \beta=93.237(5)^{\circ}$

$\mathrm{c}=14.5776(9) \AA \quad \gamma=105.501(5)^{\circ}$

1193.14(13) $\AA^{3}$

2

$1.324 \mathrm{~g} / \mathrm{m}^{3}$

$0.081 \mathrm{~mm}^{-1}$

496.0

3.392 to $61.186^{\circ}$

$-9<=\mathrm{h}<=9,-17<=\mathrm{k}<=17,-20<=\mathrm{k}<=20$

19675

$6879[\mathrm{R}(\mathrm{int})=0.0390]$

Empirical

Full-matrix least-squares on $\mathrm{F}^{2}$

6879 / 0 / 334

1.037

$\mathrm{R} 1=0.0533, \mathrm{wR} 2=0.1471$

$\mathrm{R} 1=0.0808, \mathrm{wR} 2=0.1680$

0.33 and $-0.44 \mathrm{e}^{-3}$

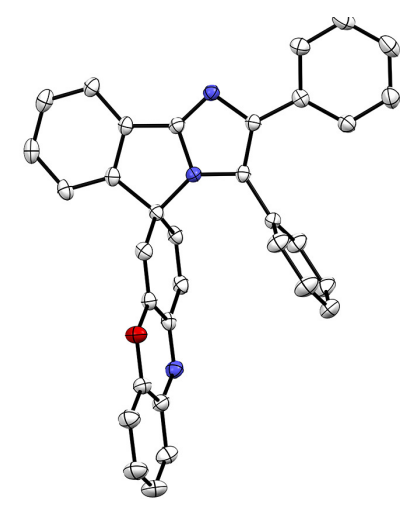

Figure S14. ORTEP representation of the molecular structure of 2 with thermal ellipsoids ( $50 \%$ probability), where oxygen and nitrogen atoms are highlighted in red and blue. 


\section{Steady-State Absorption Spectra at Different Solvents}

(a)

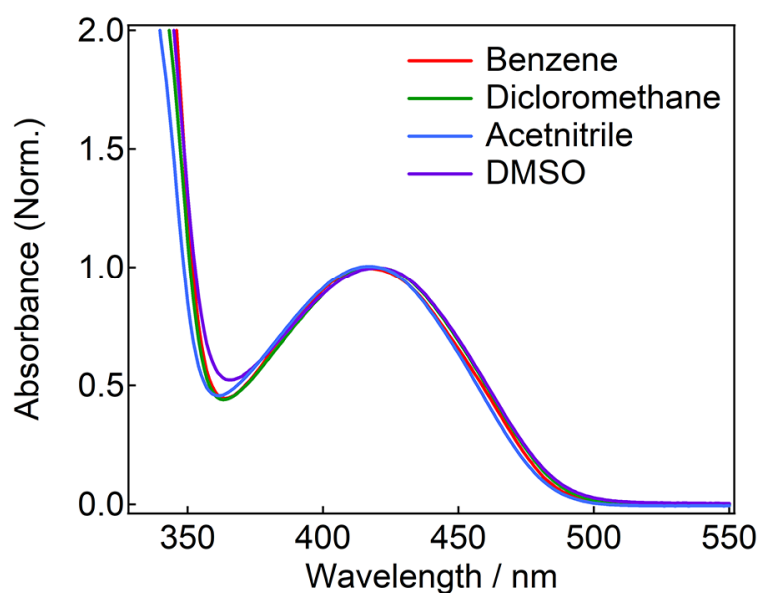

(b)

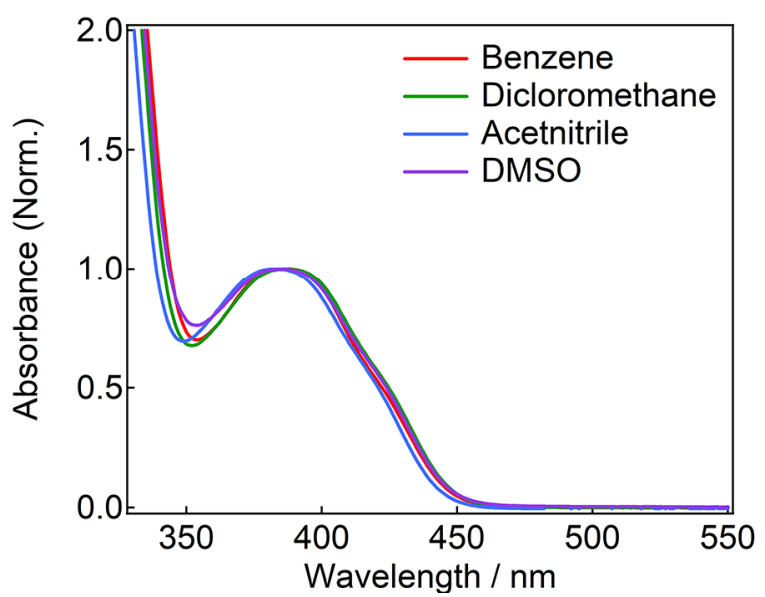

Figure S15. Normalized steady-state absorption spectra of (a) 1 and (b) 2 in different solvents.

(a)

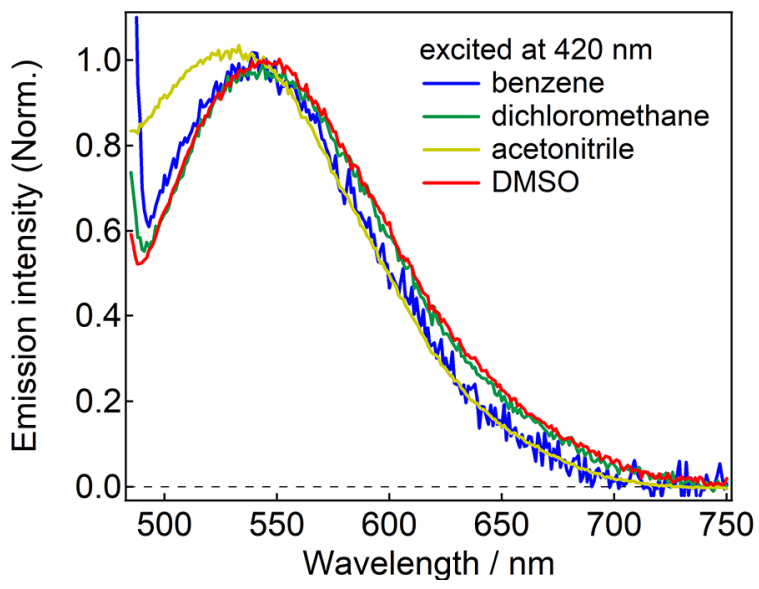

(b)

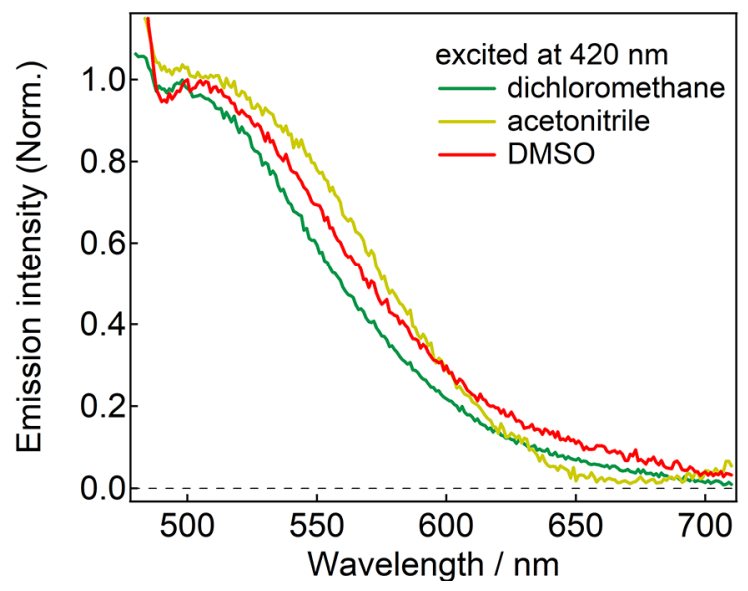

Figure S16. Normalized steady-state emission spectra of (a) 1 and (b) 2 in different solvents excited at $420 \mathrm{~nm}$. The emission spectrum of 2 in benzene could not be measured correctly because the emission quantum yield in this condition was extremely low $\left(\sim 10^{-4}\right)$. 


\section{Details of the Nanosecond to Microsecond Transient Absorption Measurements}

(a)

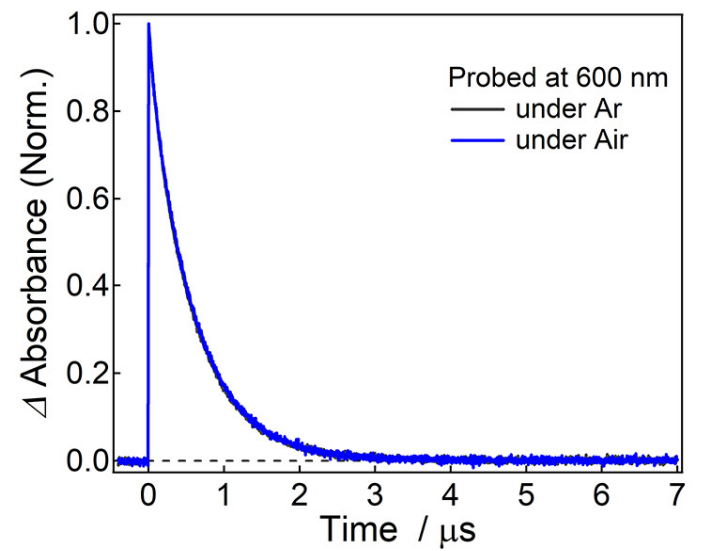

(b)

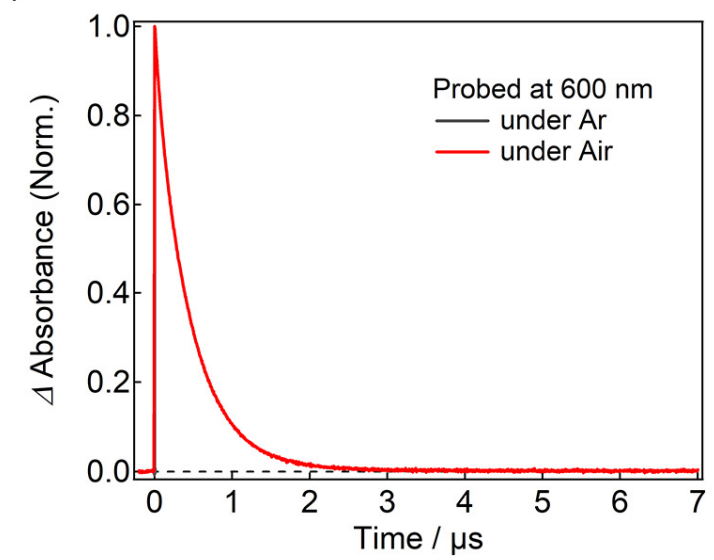

Figure S17. Normalized time profiles of the transient absorbance of (a) 1 and (b) 2 excited at $355 \mathrm{~nm}$ under argon and air atmospheres. The probed wavelength is $600 \mathrm{~nm}$ in both compounds.

(a)

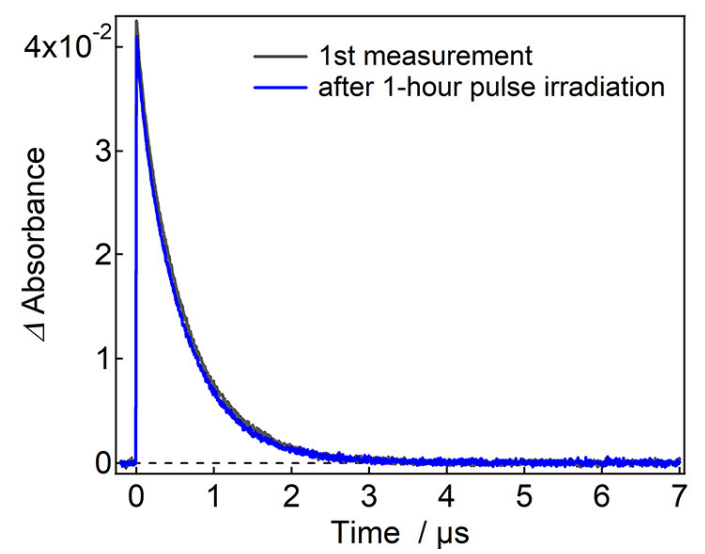

(b)

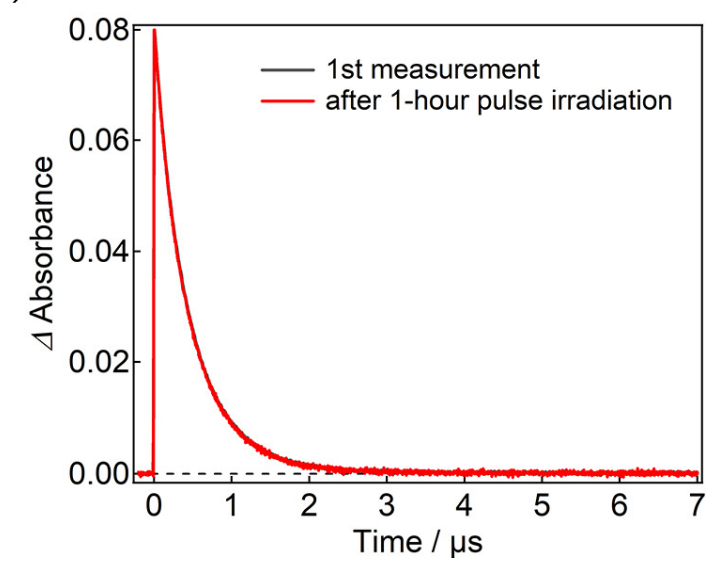

Figure S18. Effect of the repetitive irradiation of picosecond laser pulses on the time profiles of the transient absorbance of (a) 1 and (b) 2 excited at $355 \mathrm{~nm}$ under argon atmospheres. The probed wavelength is $600 \mathrm{~nm}$ in both compounds. 
(a)

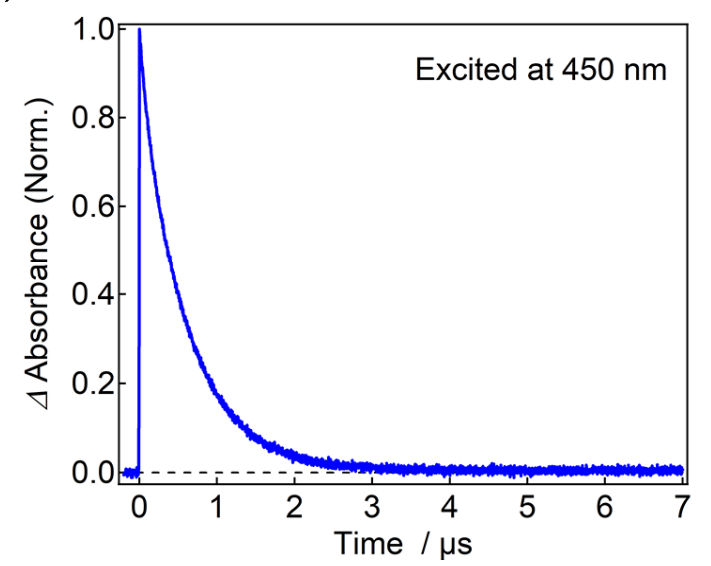

(b)

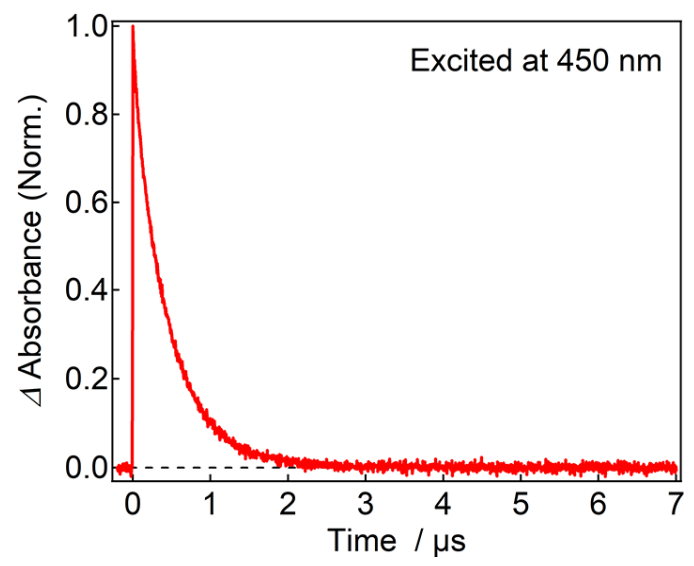

Figure S19. Normalized time profiles of the transient absorbance of (a) 1 and (b) 2 excited at $450 \mathrm{~nm}$ under argon atmosphere. The probed wavelength is $600 \mathrm{~nm}$ in both compounds.

(a)

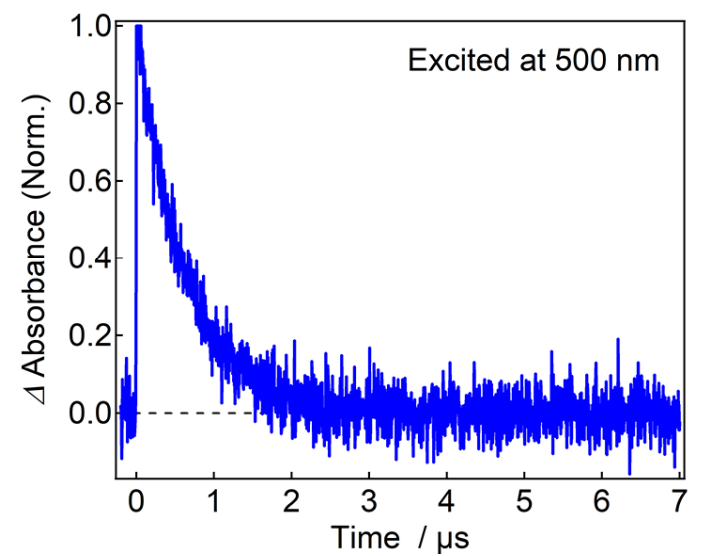

(b)

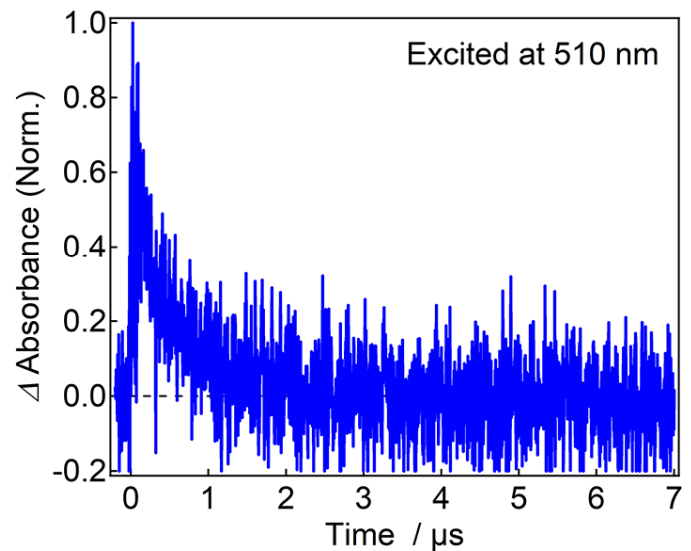

Figure S20. Normalized time profiles of the transient absorbance of 1 excited at (a) $500 \mathrm{~nm}$ and (b) $510 \mathrm{~nm}$ under argon atmosphere. The probed wavelength is $600 \mathrm{~nm}$.

(a)

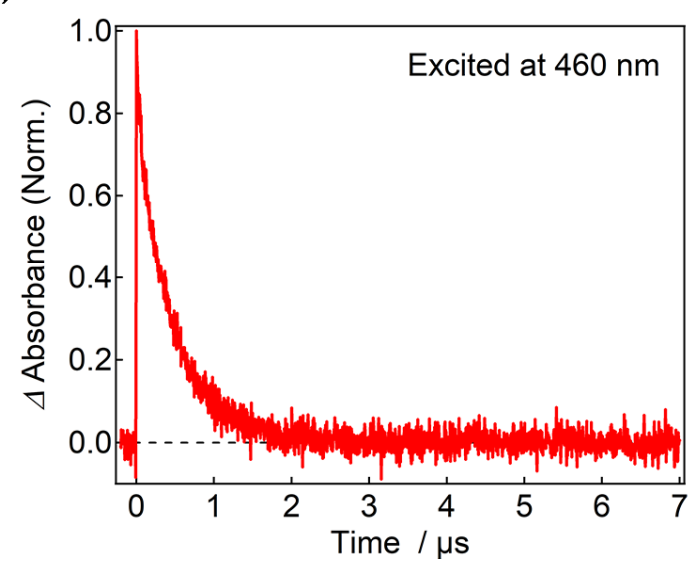

(b)

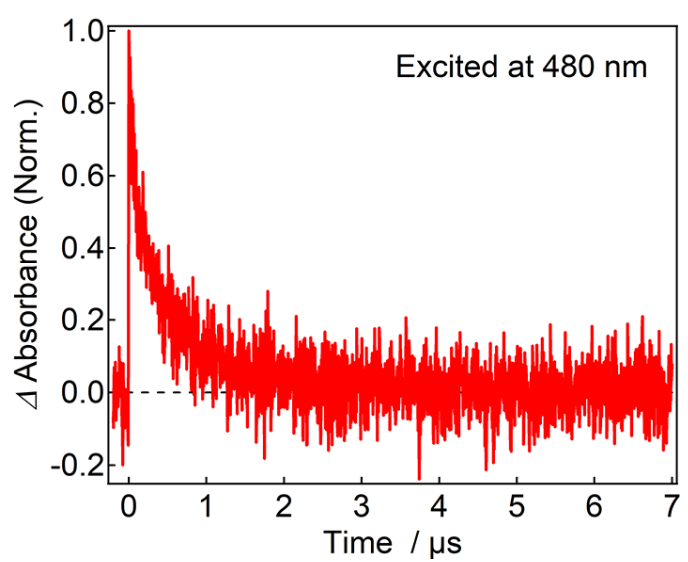

Figure S21. Normalized time profiles of the transient absorbance of 2 excited at (a) $460 \mathrm{~nm}$ and (b) $480 \mathrm{~nm}$ under argon atmosphere. The probed wavelength is $600 \mathrm{~nm}$. 


\section{Nanosecond to Microsecond Transient Absorption Spectra at Different Solvents}

(a)

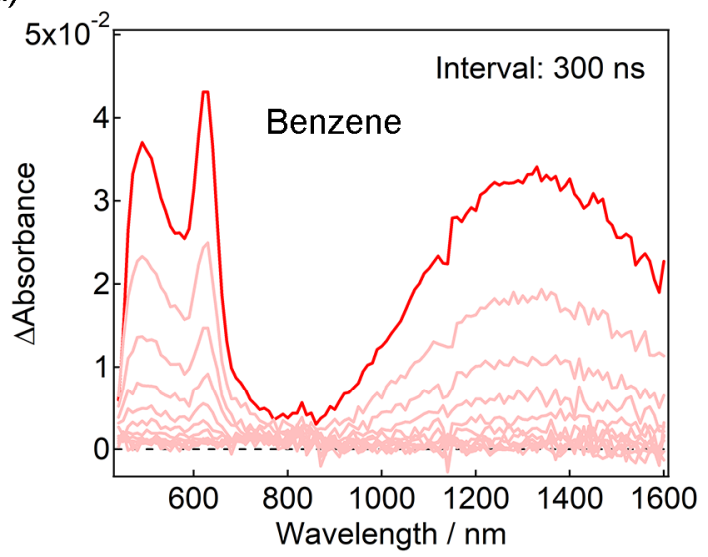

(c)

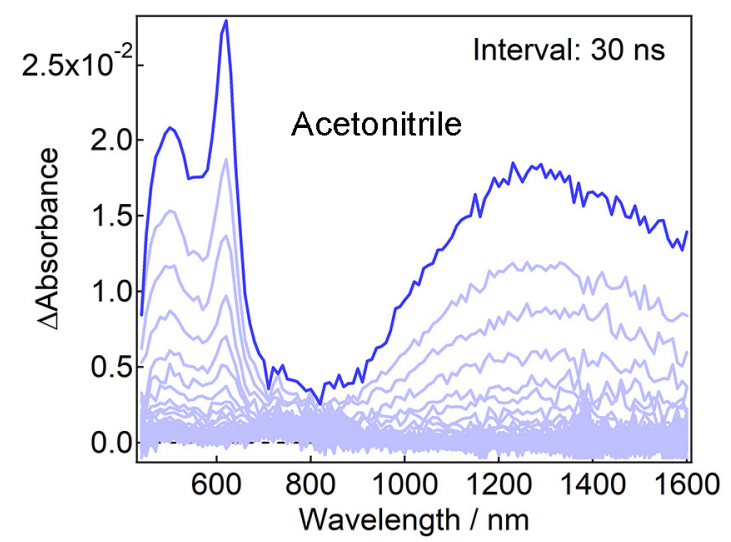

(b)

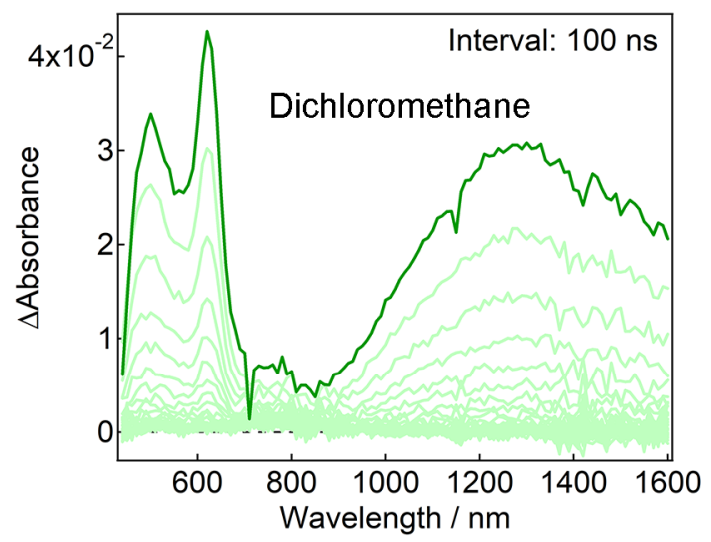

(d)

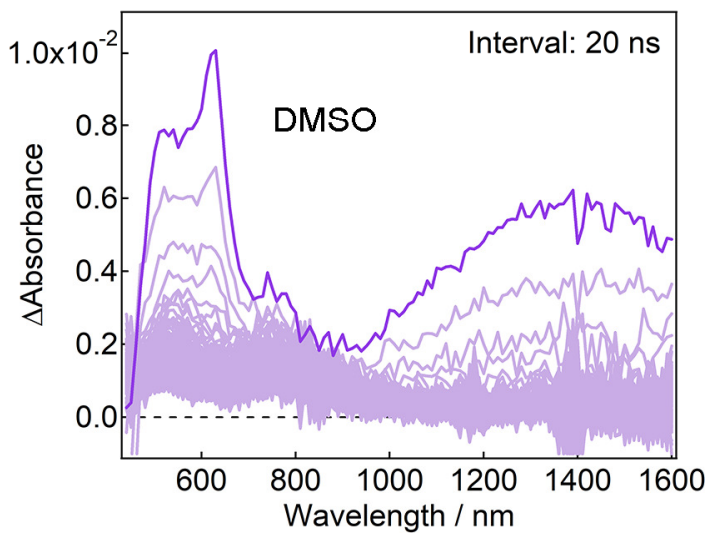

Figure S22. Time evolutions of nanosecond to microsecond transient absorption spectra of 1 in different solvents (benzene, dichloromethane, acetonitrile, dimethyl sulfoxide (DMSO) for a, b, c, and d, respectively). Transient absorption spectra of 1 in benzene and dichloromethane monotonically decay without change of the spectral shape. The transient absorption spectra of acetonitrile and DMSO show residual spectra below $800 \mathrm{~nm}$. The transient absorption dynamics in air and argon conditions revealed that the residual spectra are due to the triple excited states. 


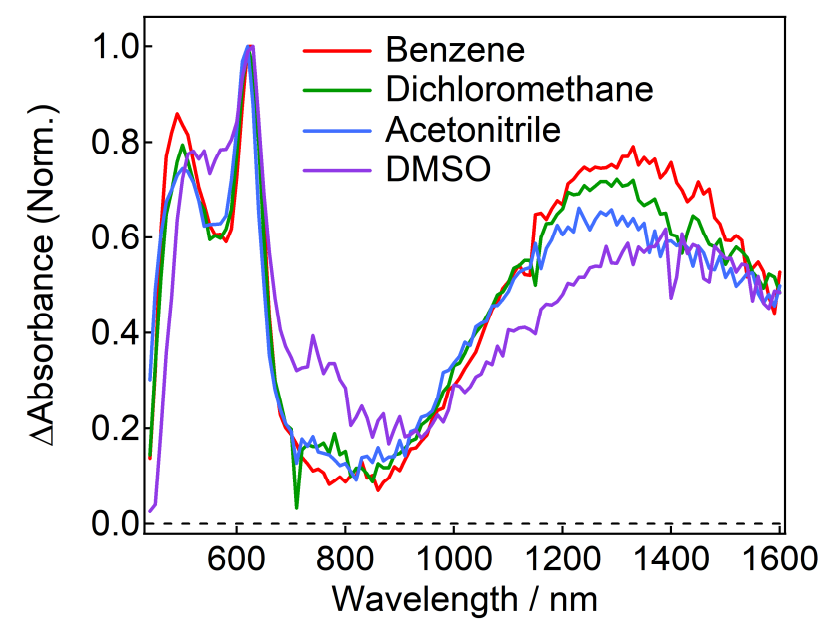

Figure S23. Normalized transient absorption spectra of 1 in different solvents at around $100 \mathrm{~ns}$. The comparison of the spectra in benzene, dichloromethane, and acetonitrile shows that the absorption spectra of the ring-opening form do not change. The spectrum in DMSO is somehow slightly different from others. One possibility for the difference may be due to the low signal to noise ratio in the spectrum in DMSO because of the rapid decay.

(a)

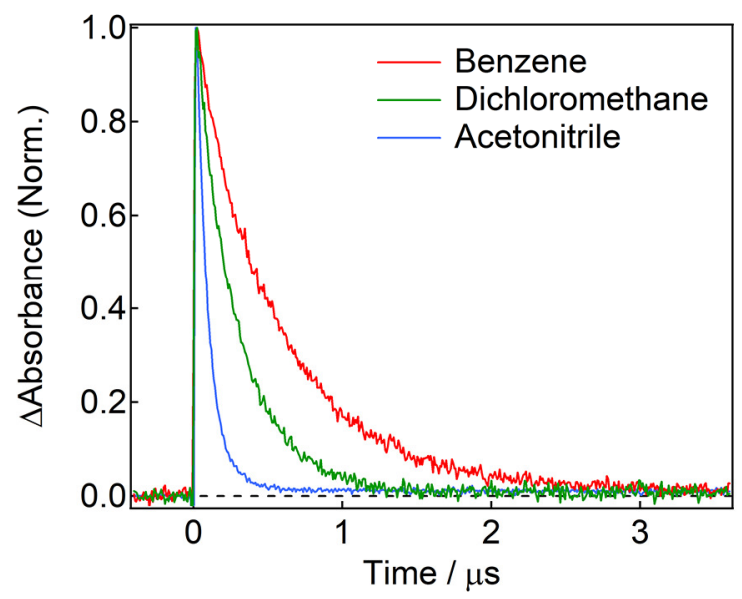

(b)

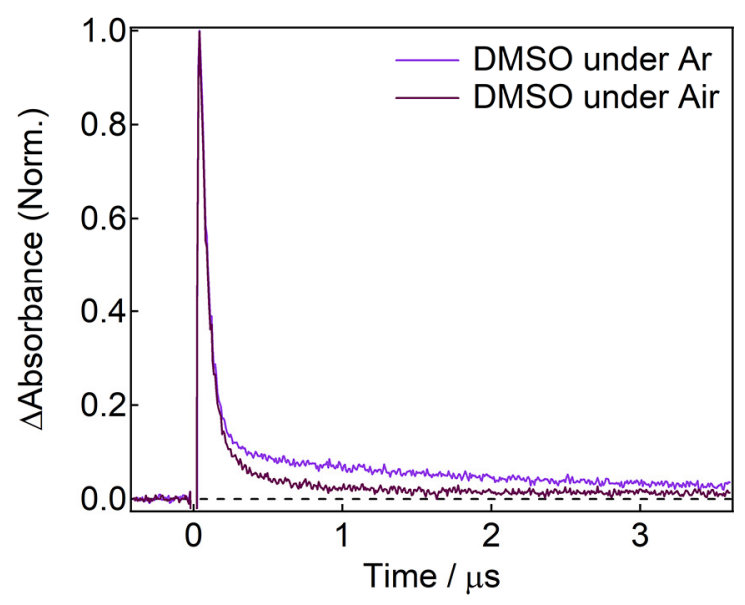

Figure S24. Nanosecond to microsecond transient absorption dynamics of 1 in different solvents. The transient absorption dynamics of 1 in DMSO in air and argon conditions revealed that the residual spectra are due to the triple excited states. 
(a)

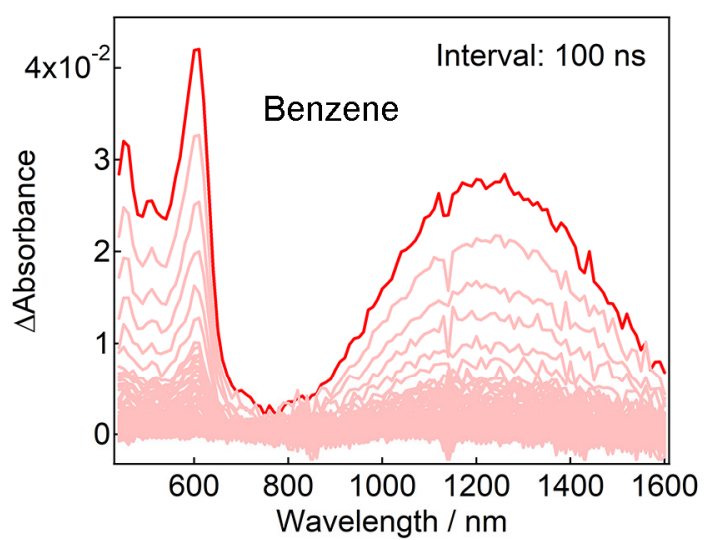

(c)

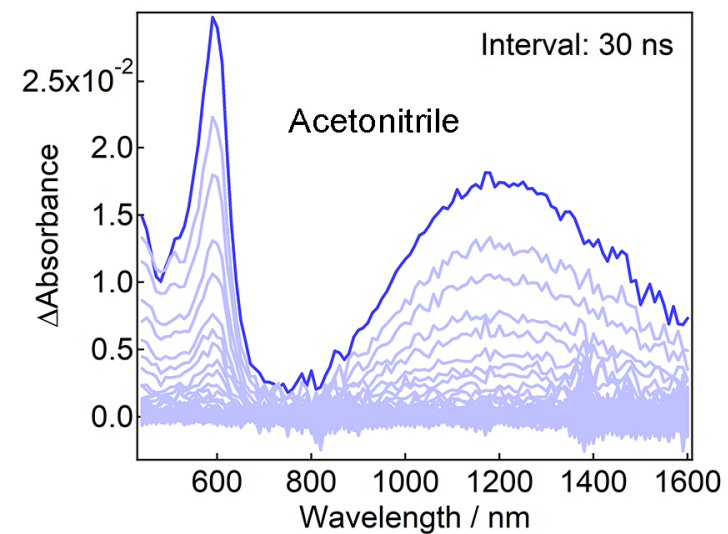

(b)

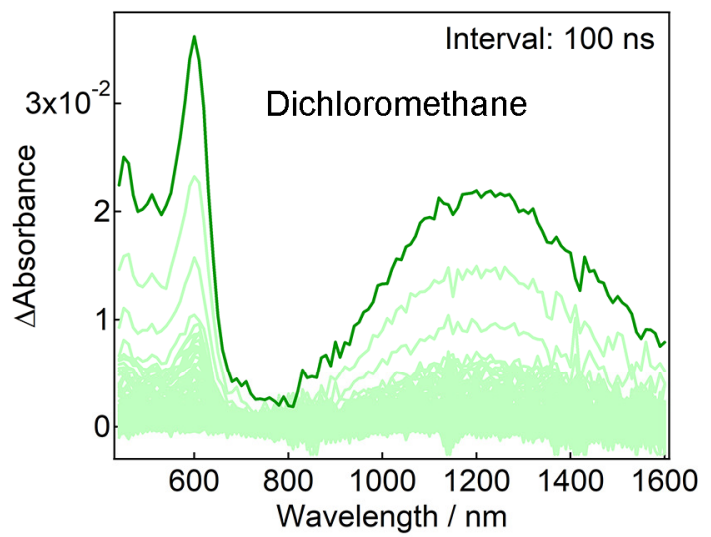

(d)

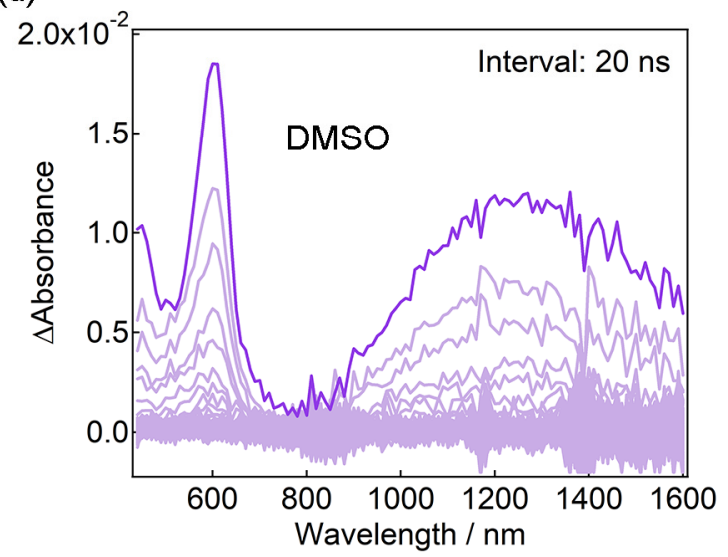

Figure S25. Time evolutions of nanosecond to microsecond transient absorption spectra of 2 in different solvents (benzene, dichloromethane, acetonitrile, dimethyl sulfoxide (DMSO) for a, b, c, and d, respectively). Transient absorption spectra of 2 in benzene, dichloromethane, and acetonitrile monotonically decay without change of the spectral shape. The transient absorption spectra of DMSO show the residual spectra below $800 \mathrm{~nm}$, which are most probably due to the triple excited states as discussed in 1. 


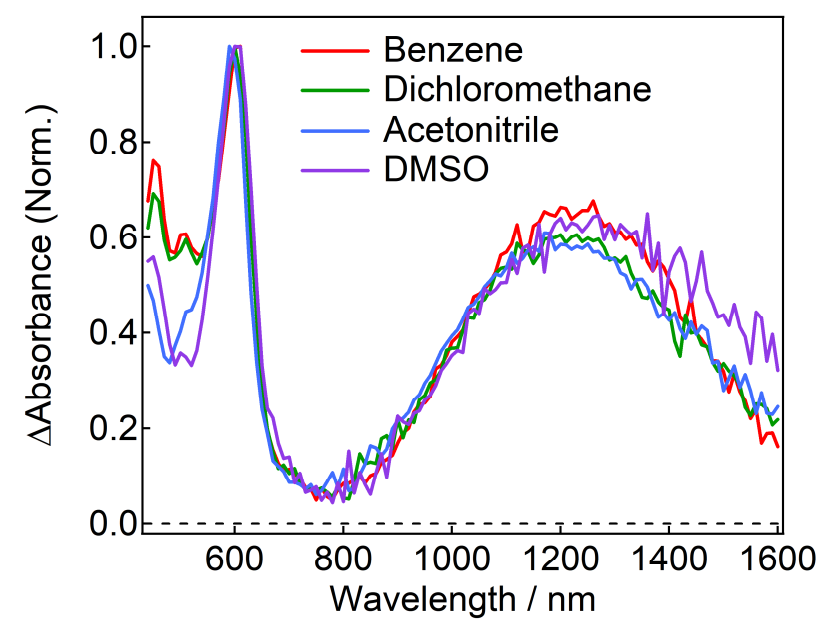

Figure S26. Normalized transient absorption spectra of 2 in different solvents at around $100 \mathrm{~ns}$. It shows that the absorption spectra of the ring-opening form do not change.

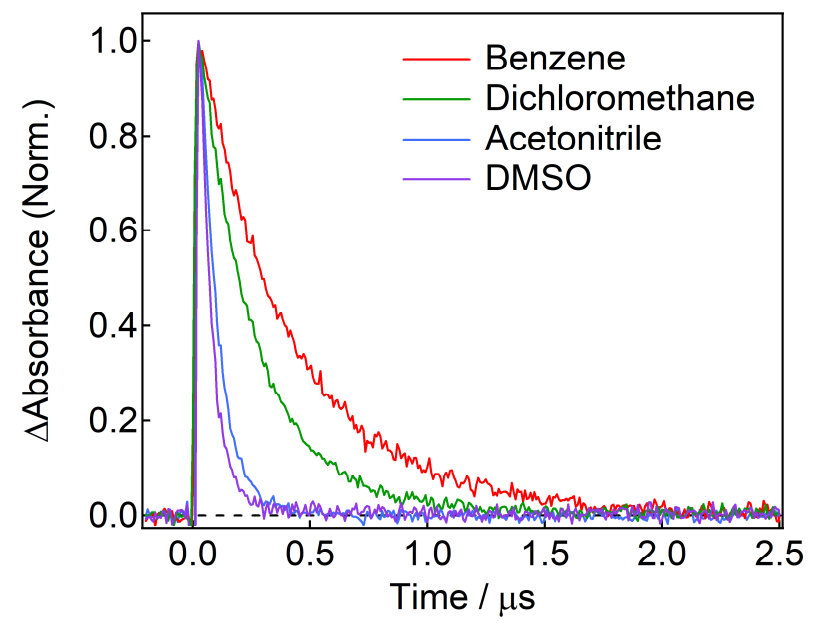

Figure S27. Nanosecond to microsecond transient absorption dynamics of 2 in different solvents. 


\section{Cyclic Voltammetry Measurements}

(a)

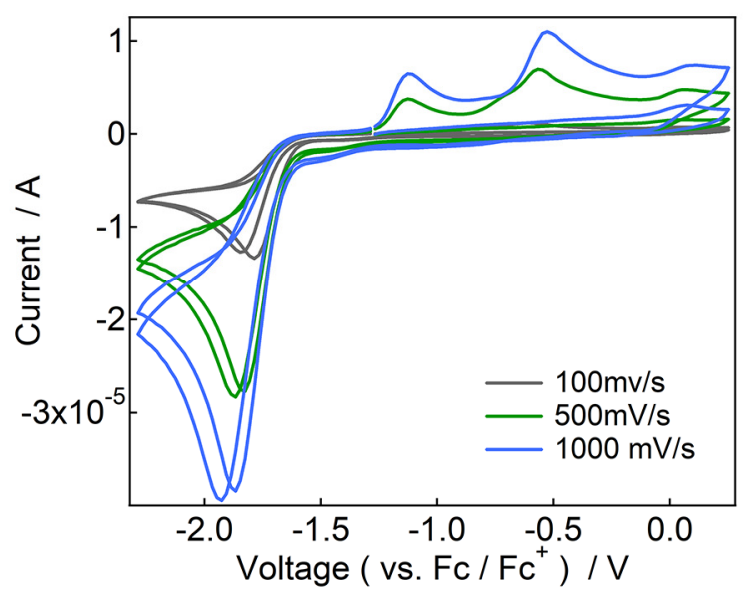

(b)

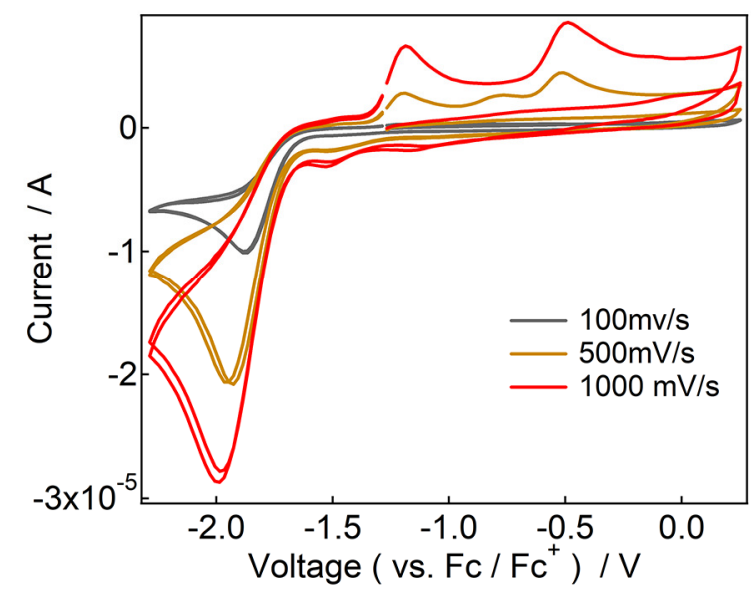

Figure S28. Cyclic voltammograms of (a) 1, and (b) 2 in dichloromethane containing $0.1 \mathrm{M}$ (TBA) $\mathrm{PF}_{6}$ as the supporting electrolyte. The concentrations are $1.1 \mathrm{mM}$ and $1.0 \mathrm{mM}$ for 1 and 2 , respectively. Potential scan rate was set to $1 \mathrm{~V} / \mathrm{s}$.

(a)

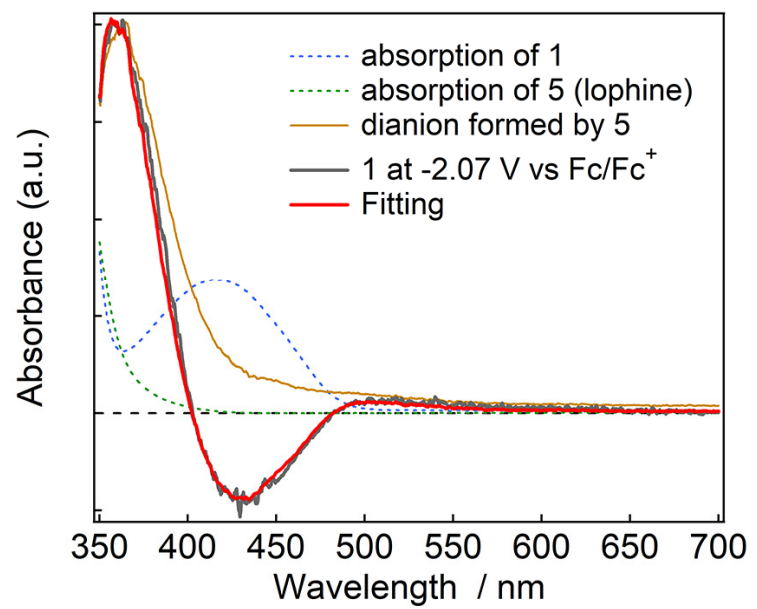

(b)

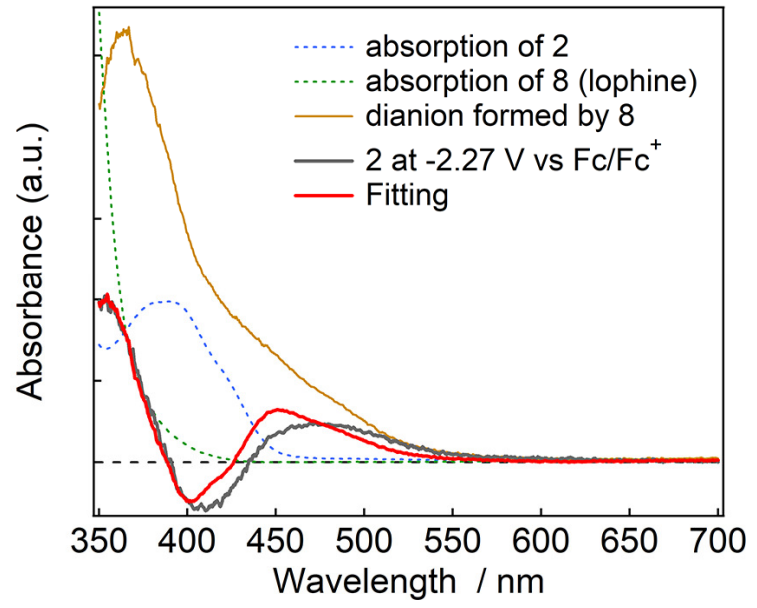

Figure S29. (a) The differential absorption spectra of 1 (gray) before and after the constant potential at $-2.07 \mathrm{~V}$ versus $\mathrm{Fc} / \mathrm{Fc}^{+}$for $40 \mathrm{~s}$ and the absorption spectra of 5 (precursor of 1) and the dianion of 5 formed by adding TBAOH. (b) The differential absorption spectra of 2 (gray) before and after the constant potential at $-2.07 \mathrm{~V}$ versus $\mathrm{Fc} / \mathrm{Fc}^{+}$for $40 \mathrm{~s}$ and the absorption spectra of 8 (precursor of 2) and the dianion of 8 formed by adding TBAOH. The fitting is based on the superposition of the bleach of the absorption spectrum of 1 (and 2) and the absorption spectra of dianions of 5 (and 8). 


\section{Femtosecond to Nanosecond Transient Absorption Measurements}

(a)

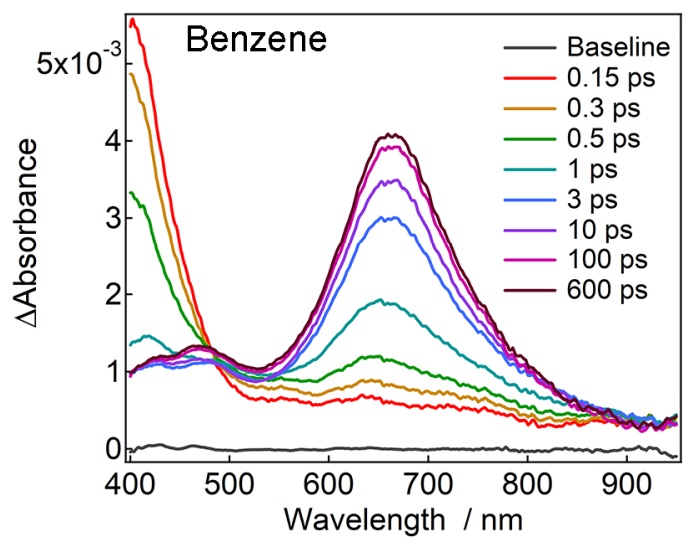

(b)

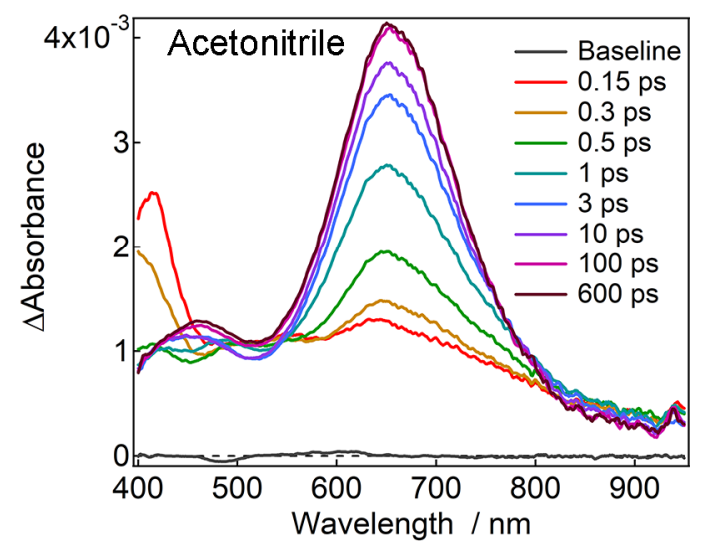

Figure S30. Time evolutions of femtosecond to nanosecond transient absorption spectra of PIC in (a) benzene and (b) acetonitrile.

(a)

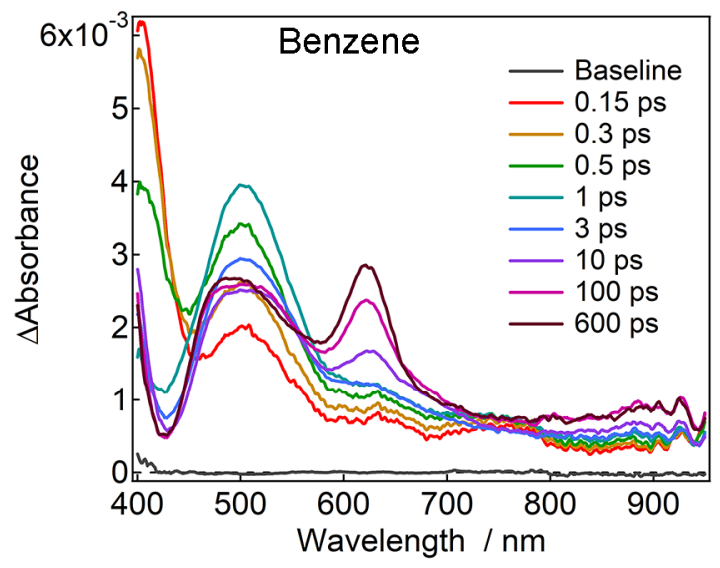

(c)

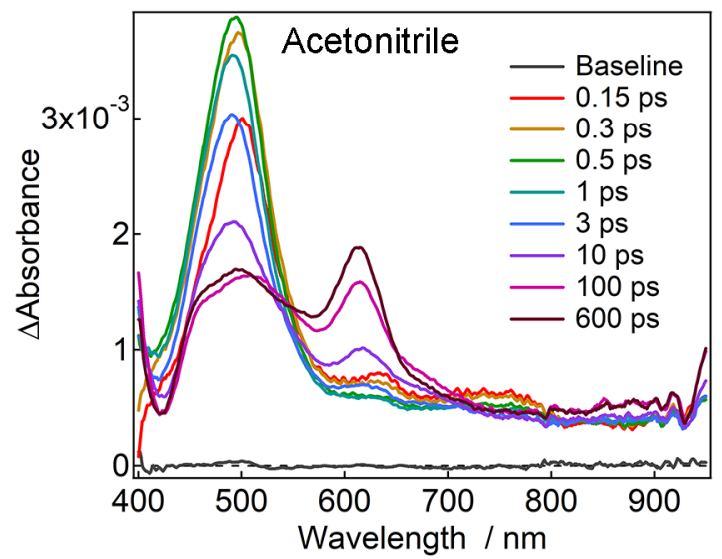

(b)

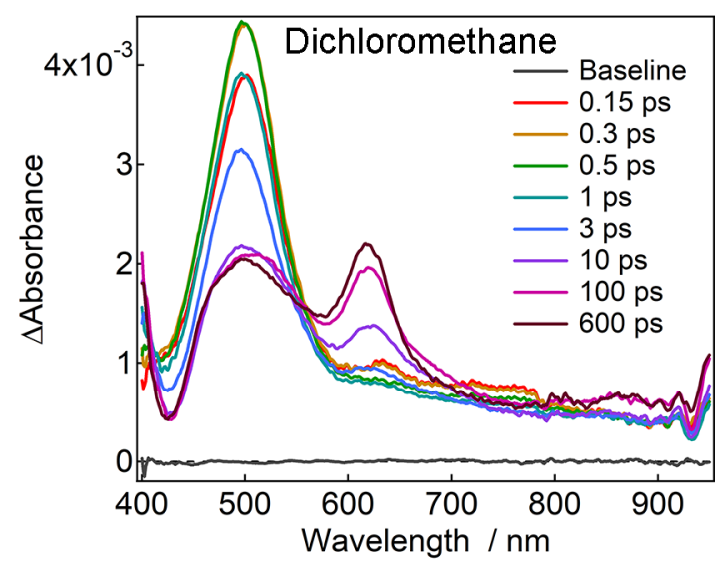

(d)

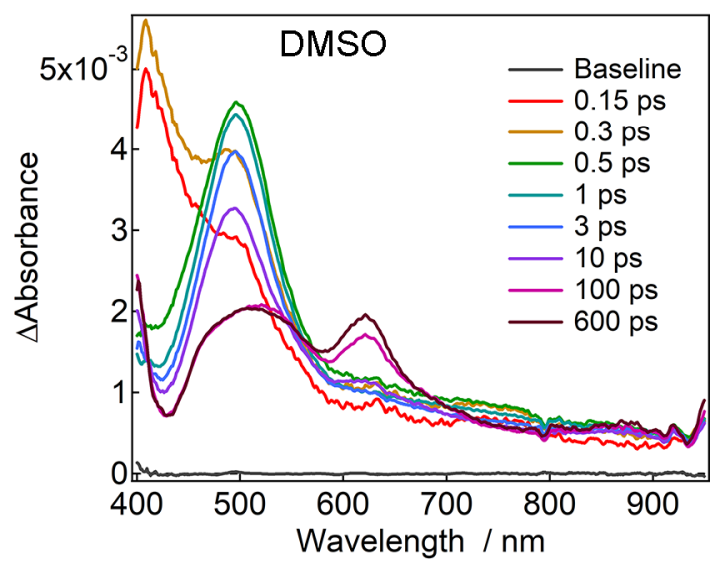

Figure S31. Time evolutions of femtosecond to nanosecond transient absorption spectra of 1 in different solvents (benzene, dichloromethane, acetonitrile, and DMSO for a, b, c, and d, respectively). 
(a)

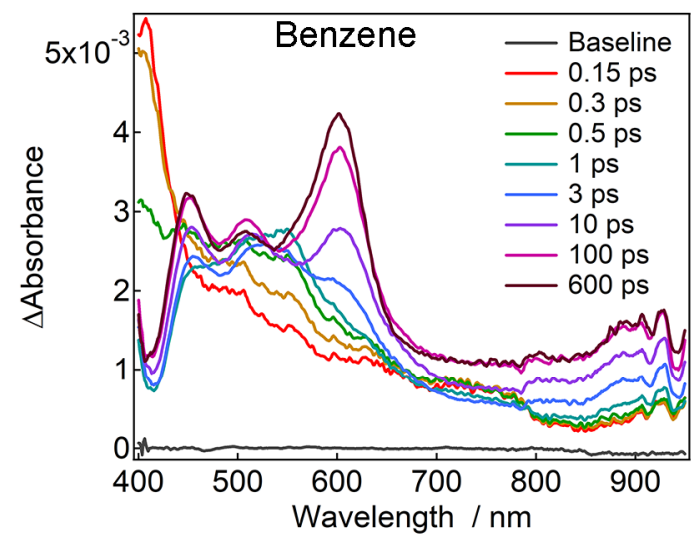

(c)

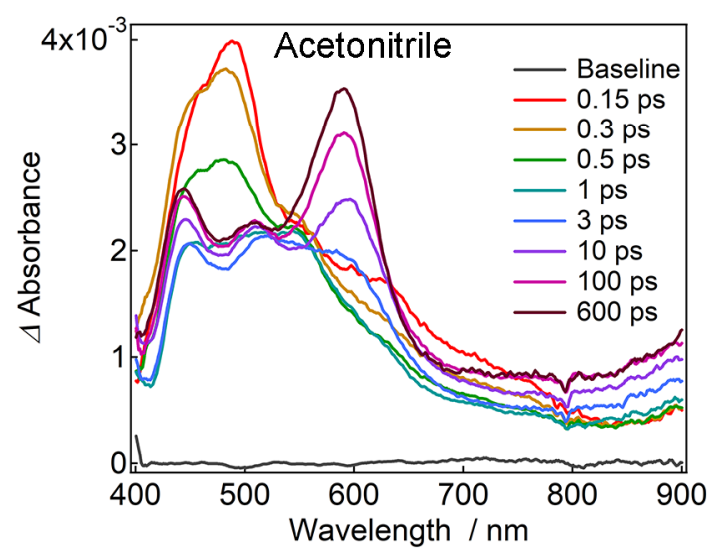

(b)

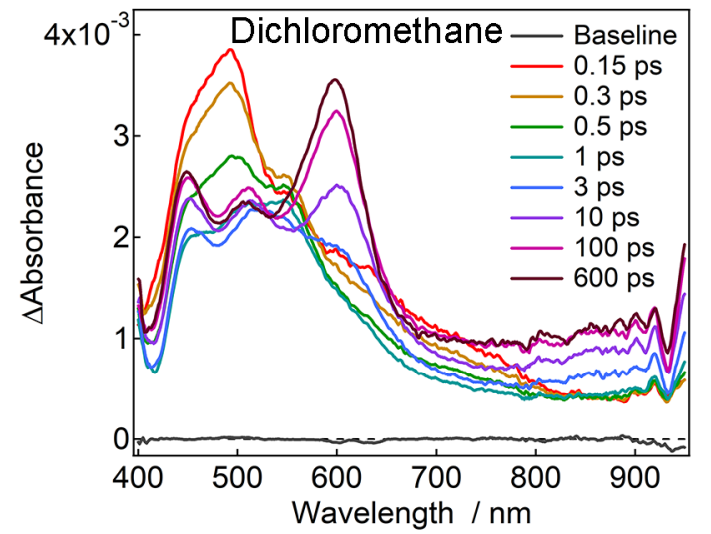

(d)

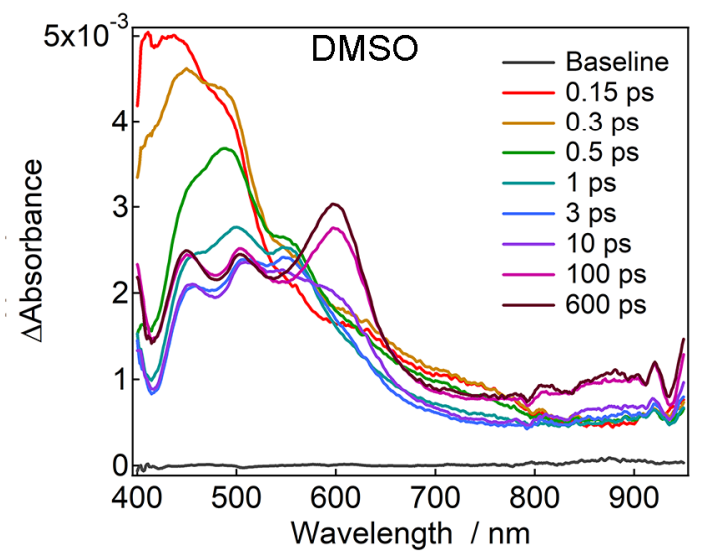

Figure S32. Time evolutions of femtosecond to nanosecond transient absorption spectra of 2 in different solvents (benzene, dichloromethane, acetonitrile, and DMSO for a, b, c, and d, respectively).

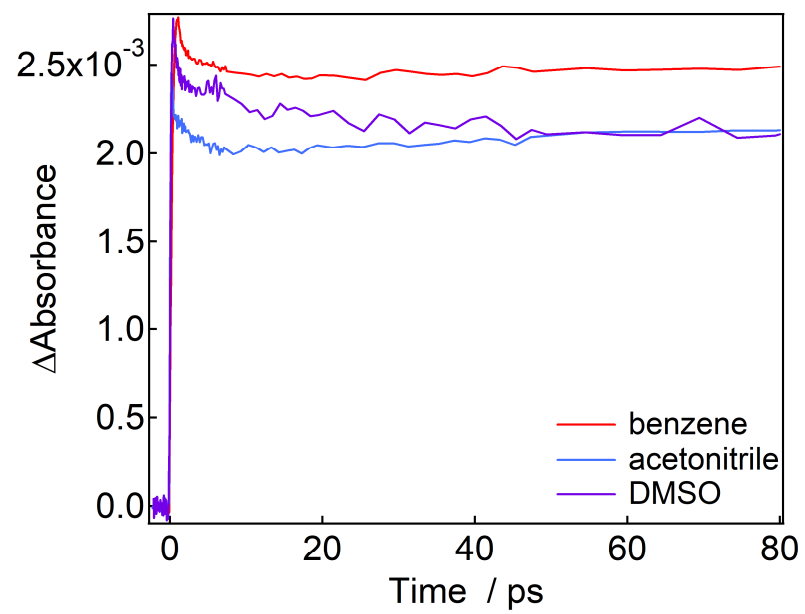

Figure S33. Transient absorption dynamics of 2 in different solvents. 
(a)

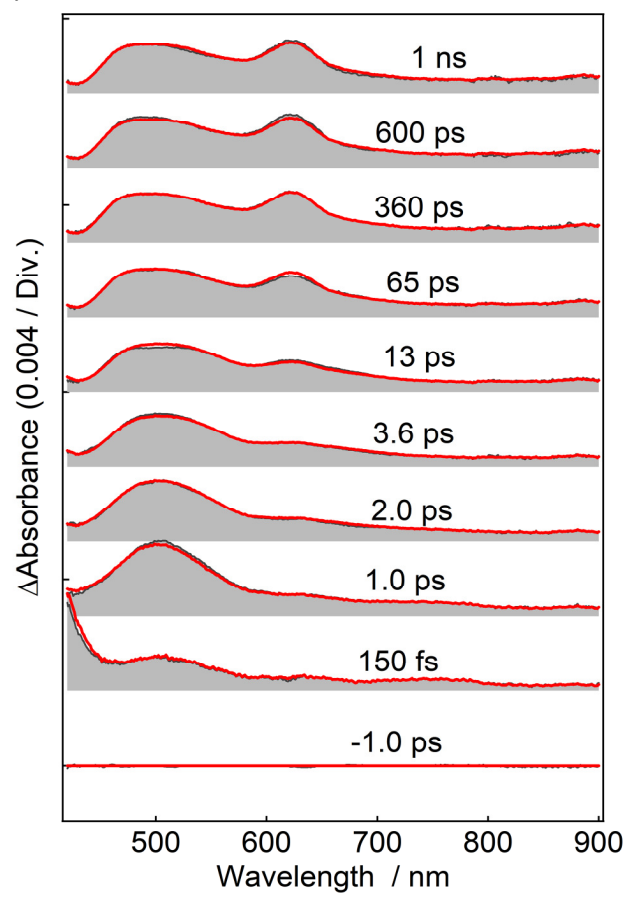

(b)

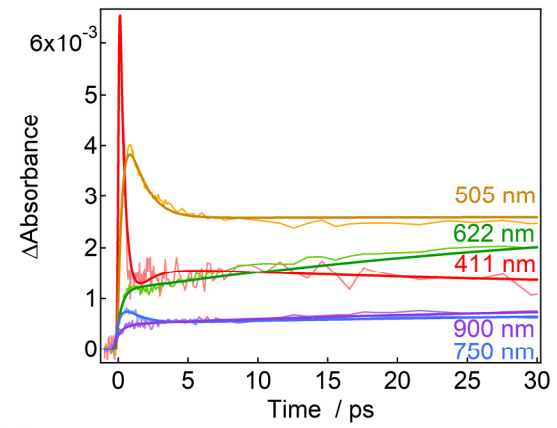

(c)

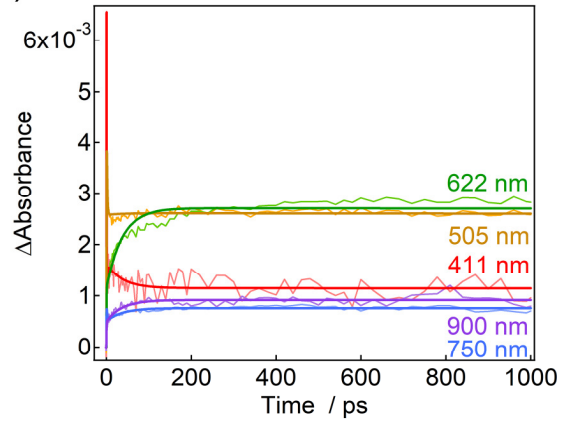

Figure S34. Time evolutions of femtosecond to nanosecond transient absorption spectra of 1 in benzene excited at $360 \mathrm{~nm}$.

(a)

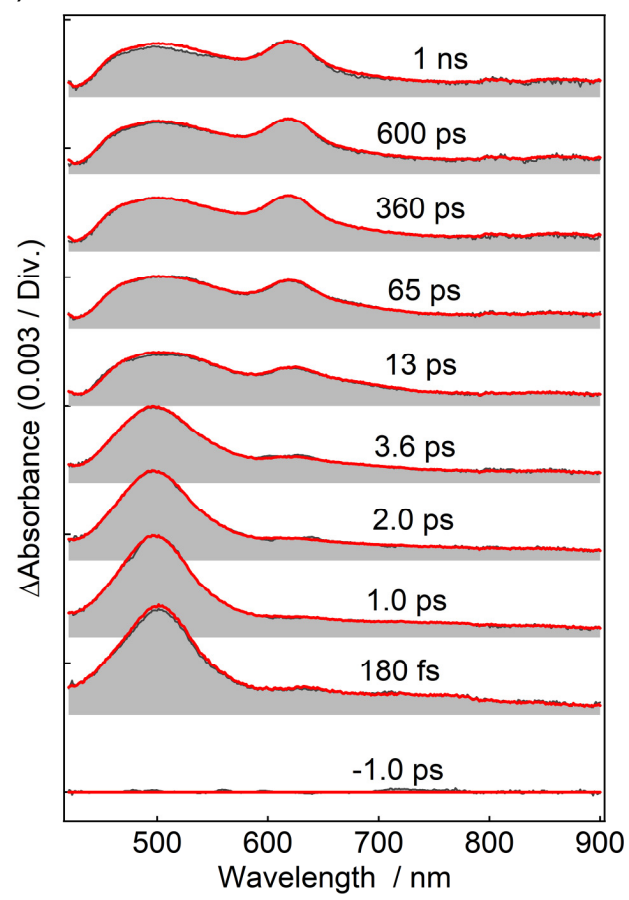

(b)

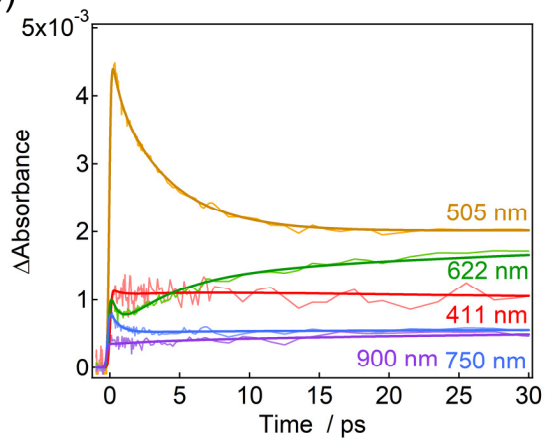

(c)

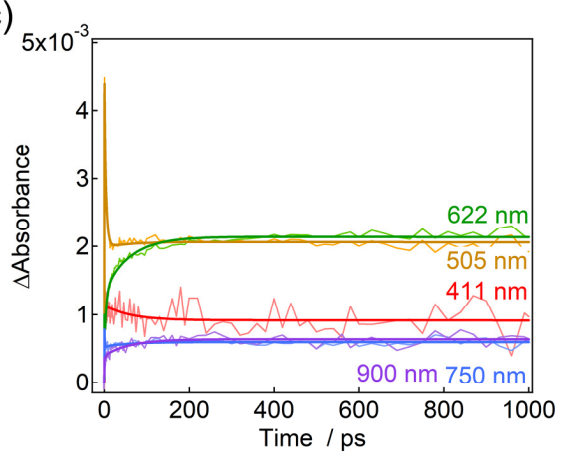

Figure S35. Time evolutions of femtosecond to nanosecond transient absorption spectra of 1 in dichloromethane excited at $360 \mathrm{~nm}$. 
(a)

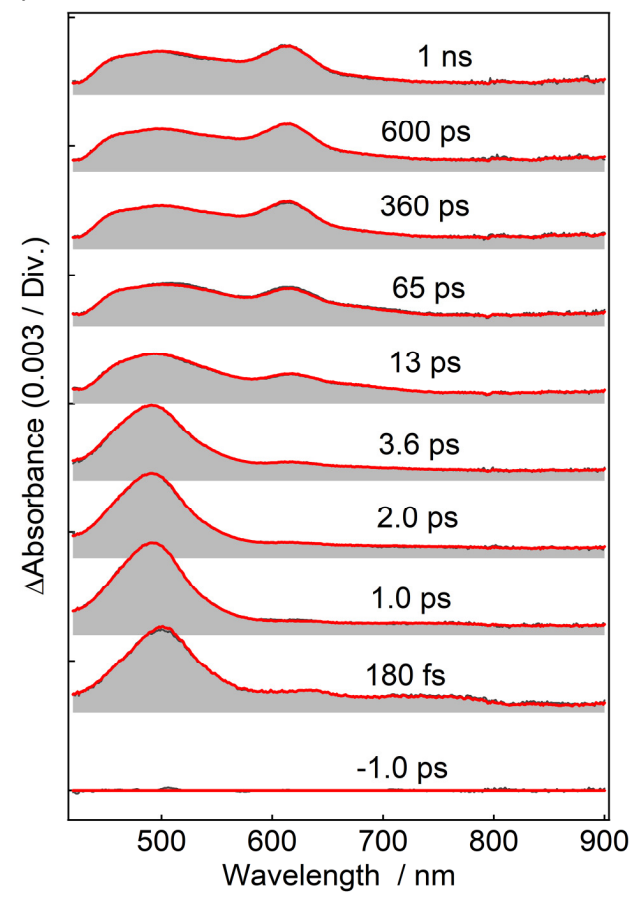

(b)

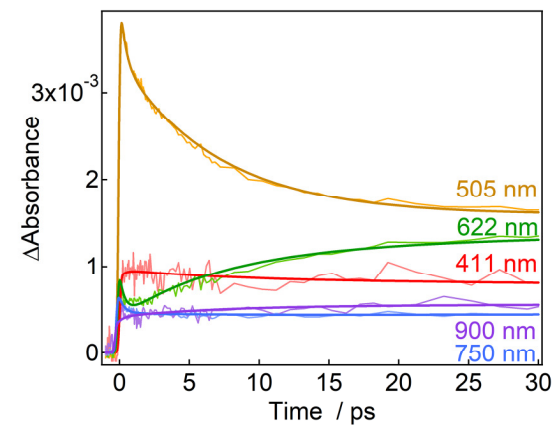

(c)

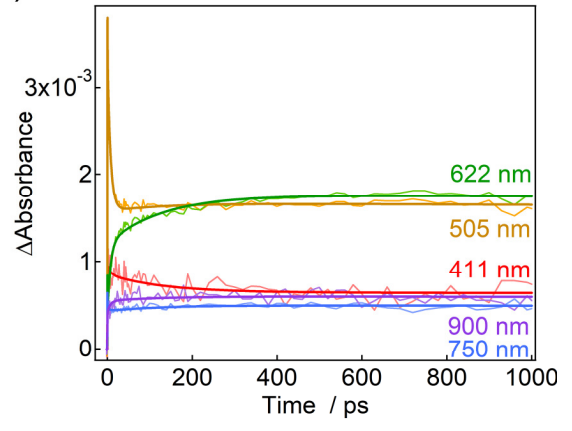

Figure S36. Time evolutions of femtosecond to nanosecond transient absorption spectra of 1 in acetonitrile excited at $360 \mathrm{~nm}$.

(a)

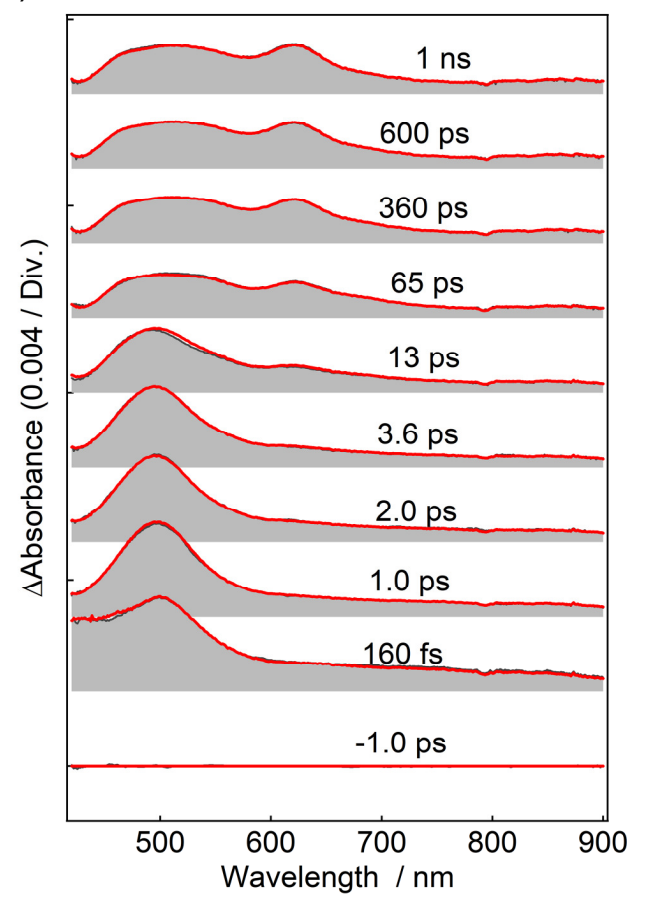

(b)

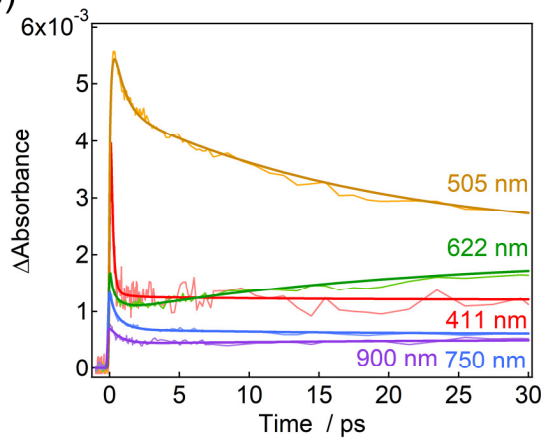

(c)

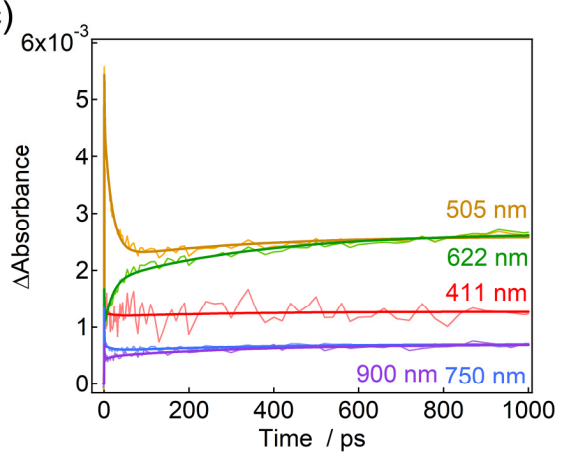

Figure S37. Time evolutions of femtosecond to nanosecond transient absorption spectra of 1 in DMSO excited at $360 \mathrm{~nm}$. 
(a)

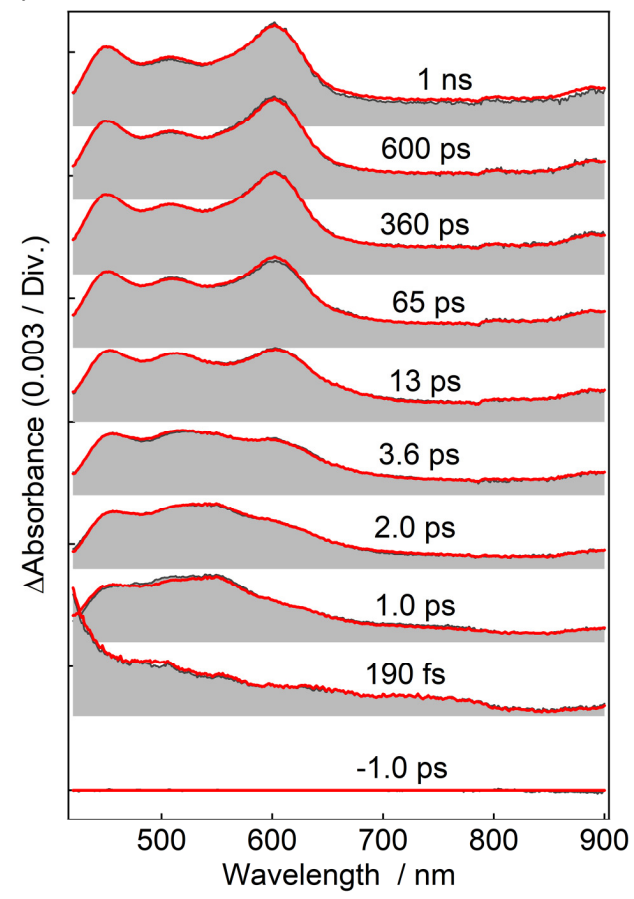

(b)

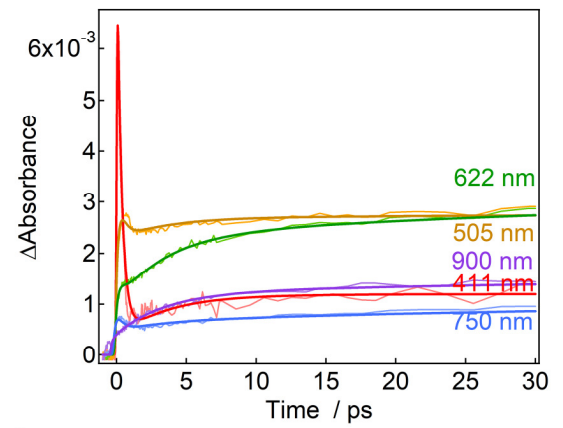

(c)

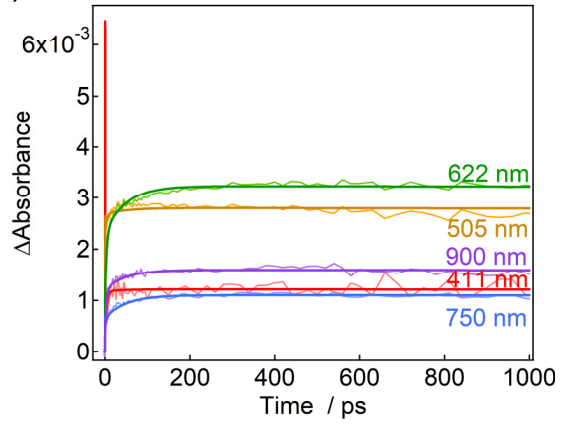

Figure S38. Time evolutions of femtosecond to nanosecond transient absorption spectra of 2 in benzene excited at $360 \mathrm{~nm}$.

(a)

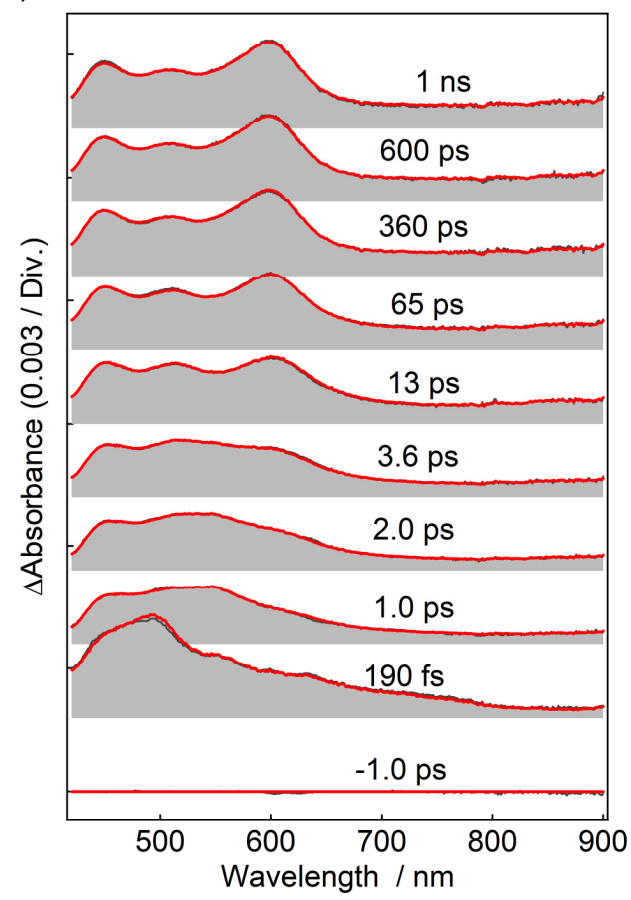

(b)

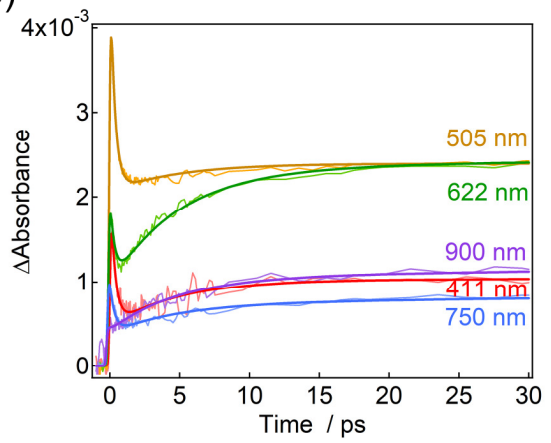

(c)

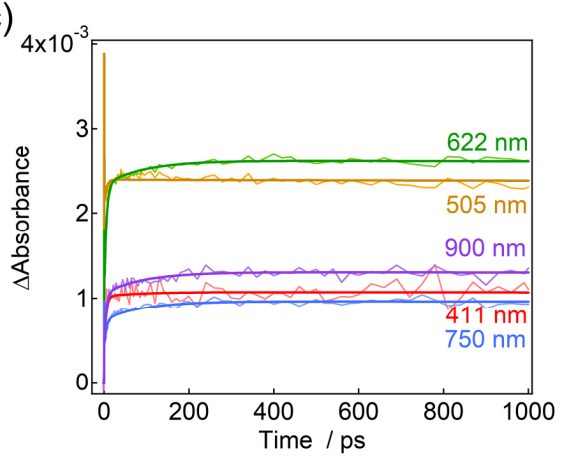

Figure S39. Time evolutions of femtosecond to nanosecond transient absorption spectra of 2 in dichloromethane excited at $360 \mathrm{~nm}$. 
(a)

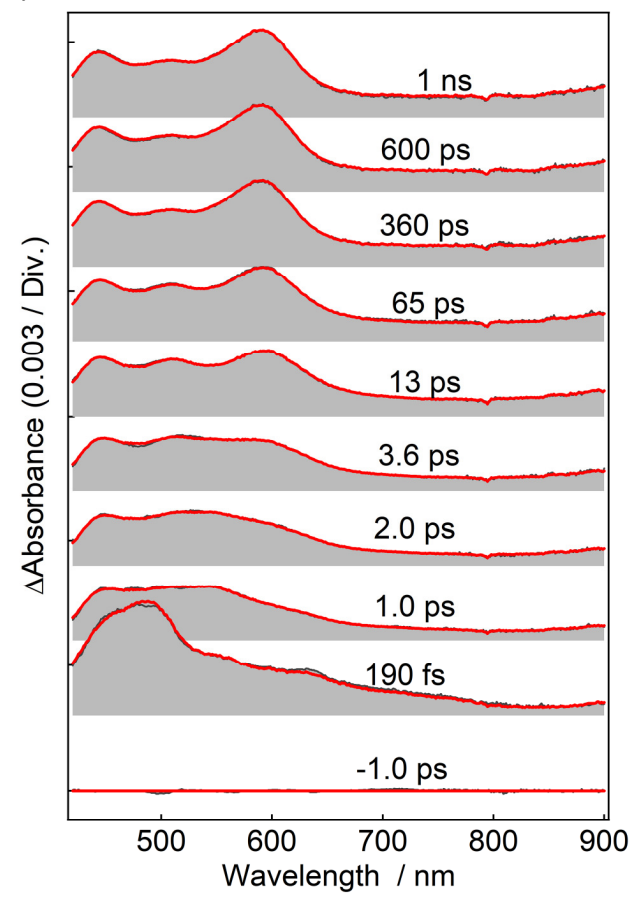

(b)

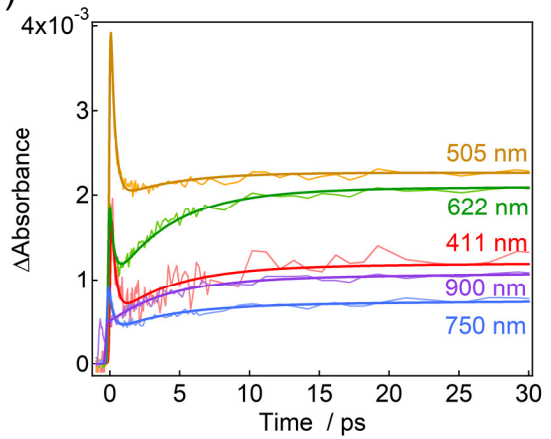

(c)

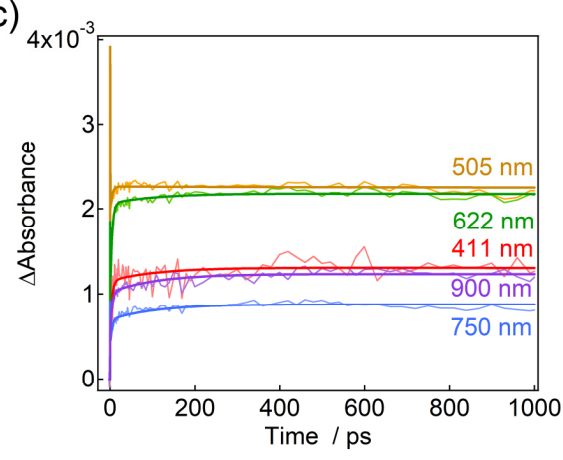

Figure S40. Time evolutions of femtosecond to nanosecond transient absorption spectra of 2 in acetonitrile excited at $360 \mathrm{~nm}$.

(a)

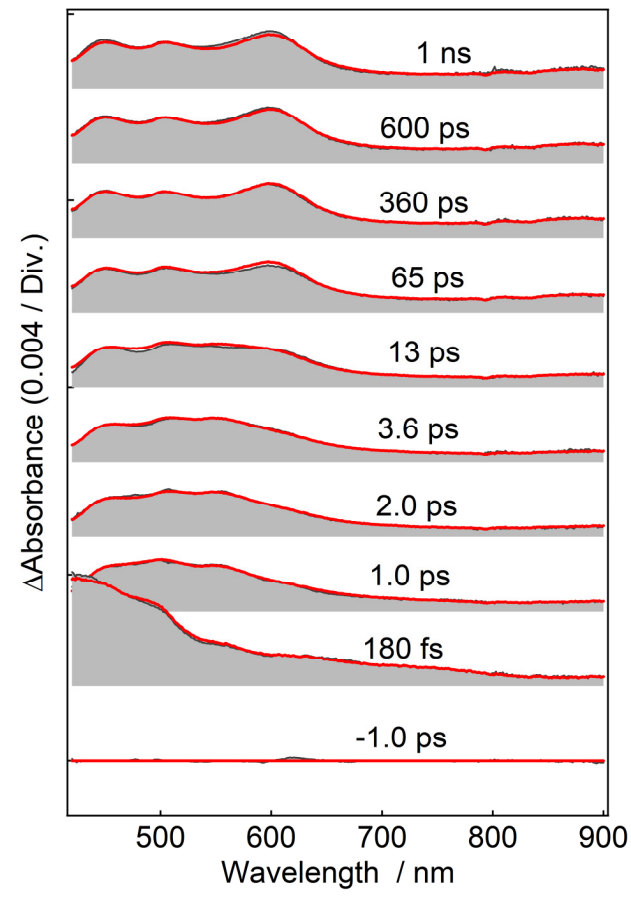

(b)

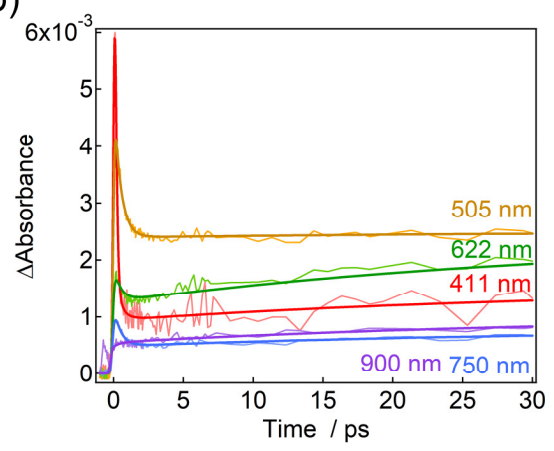

(c)

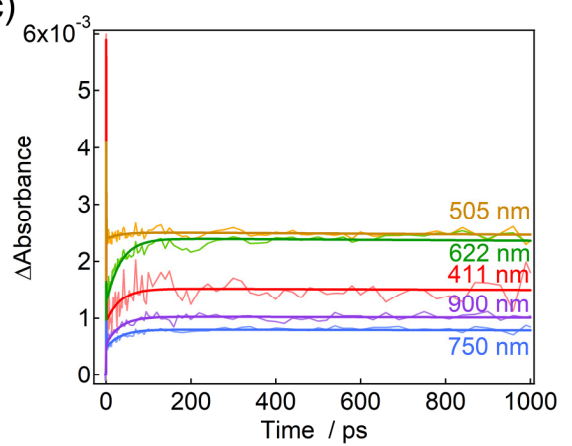

Figure S41. Time evolutions of femtosecond to nanosecond transient absorption spectra of 2 in DMSO excited at $360 \mathrm{~nm}$. 
(a)

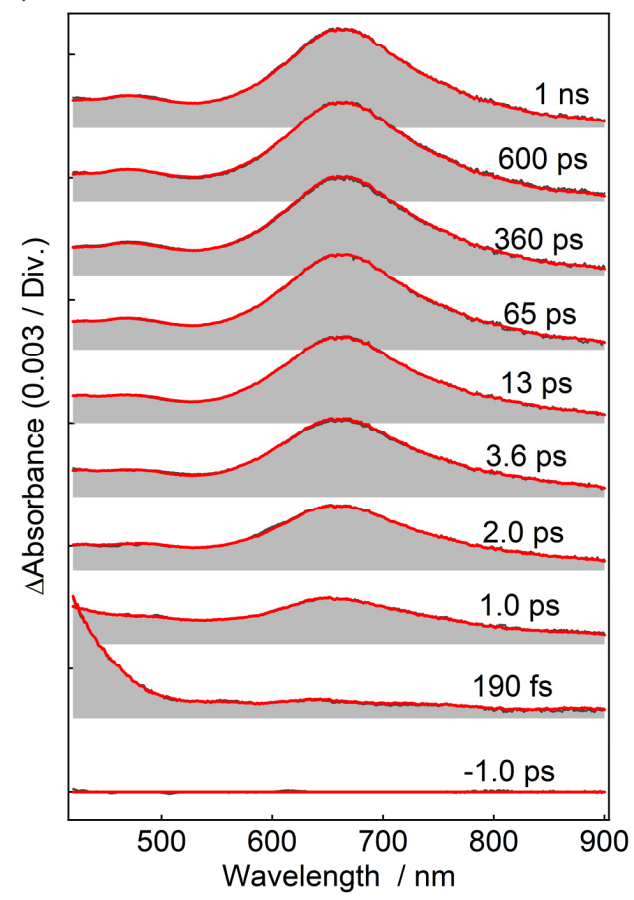

(b)

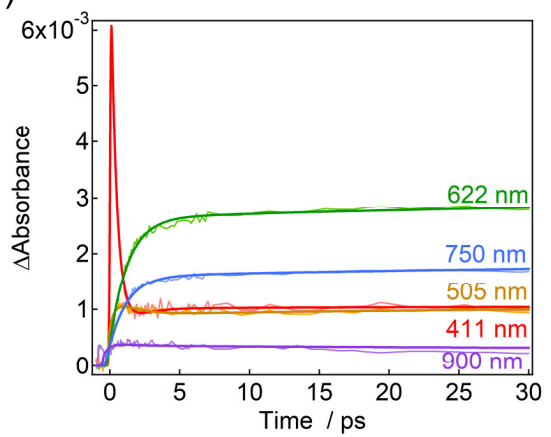

(c)

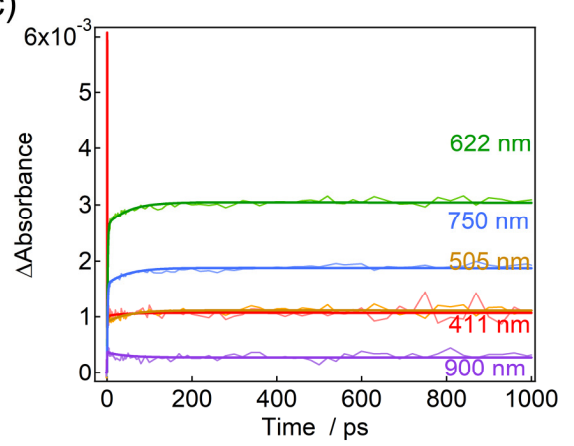

Figure S42. Time evolutions of femtosecond to nanosecond transient absorption spectra of PIC in benzene excited at $330 \mathrm{~nm}$.

(a)

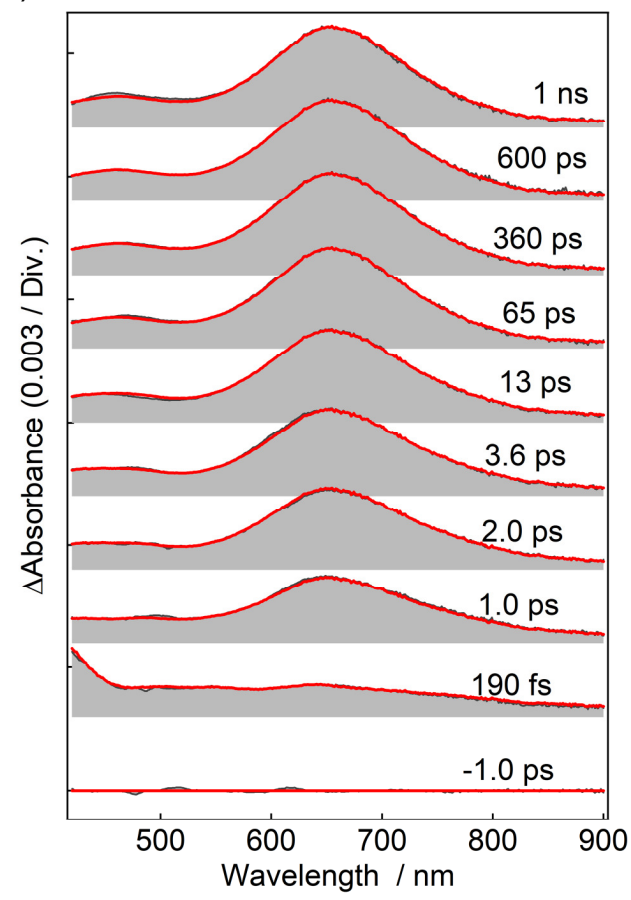

(b)

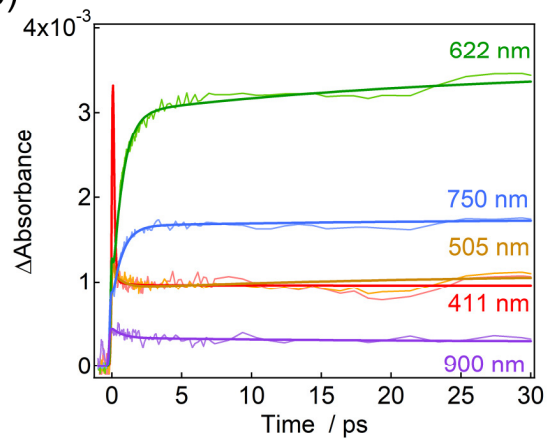

(c)

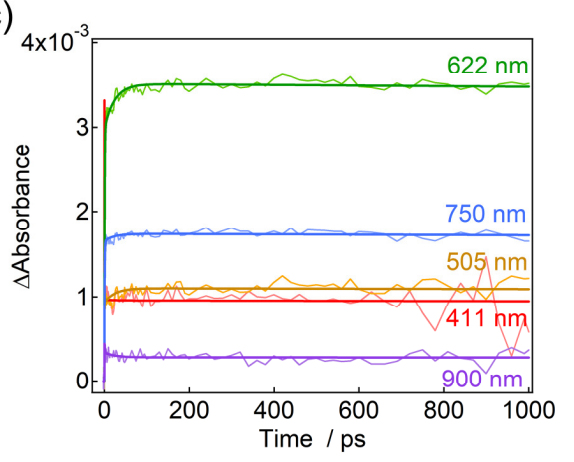

Figure S43. Time evolutions of femtosecond to nanosecond transient absorption spectra of PIC in acetonitrile excited at $330 \mathrm{~nm}$. 
(a)

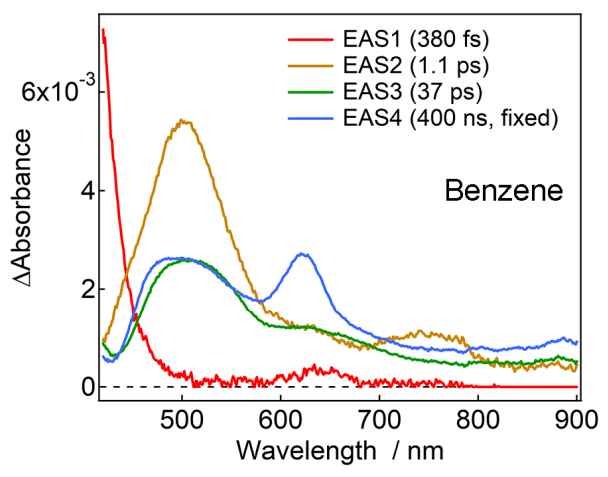

(c)

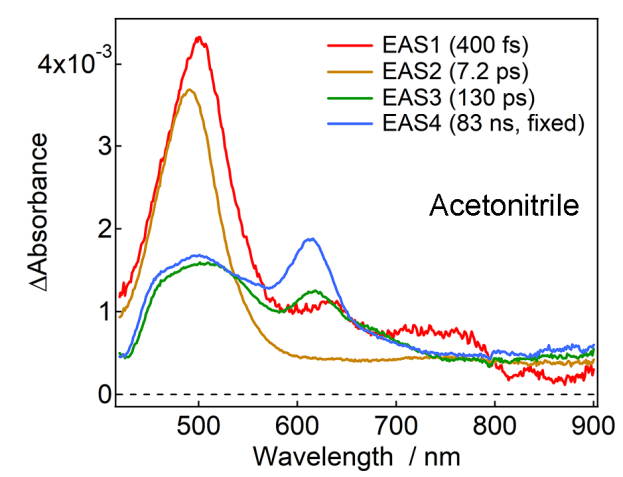

(b)

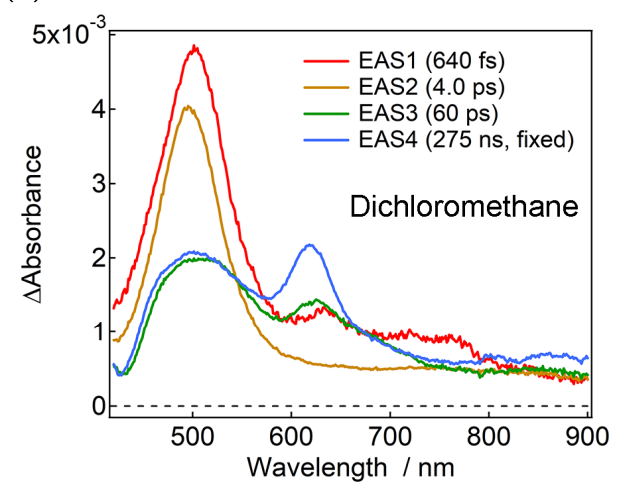

(d)

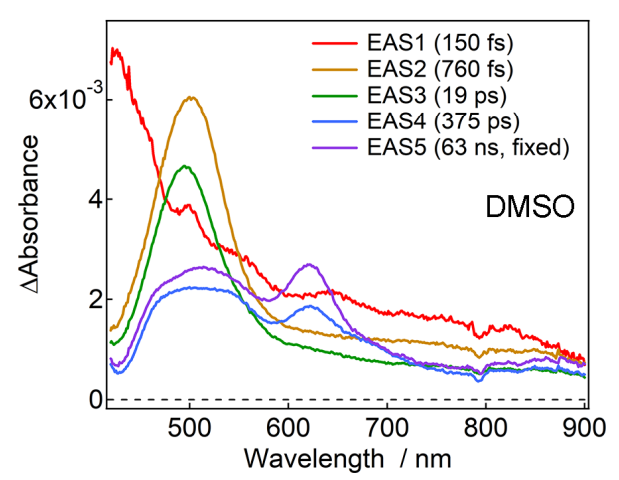

Figure S44. Evolution associated spectra (EAS) of 1 in different solvents excited at $360 \mathrm{~nm}$. The transient absorption spectra of 1 in DMSO were analyzed with a 5-state sequential decay model because it was difficult to fit the initial decay process with a 4-state sequential decay model. EAS1s in benzene and DMSO are most probably assigned to the $\mathrm{S}_{1}$ state of the closed form. On the other hand, the $\mathrm{S}_{1}$ state was not observed in dichloromethane and acetonitrile most probably due to the acceleration of the bond cleavage process as was shown in EASs of PIC (Figure S44). The spectral shape of EAS1s of dichloromethane and acetonitrile are almost identical to those of EAS2s (EAS2 and EAS3 in DMSO). This may indicate that there are two relaxation pathways from the photogenerated charge transfer state. One process if the formation of the open-ring form, and the other process may be the geminate recombination to form the triplet excited state of the closed form as was observed in nanosecond transient absorption spectroscopy in polar solvents. 
(a)

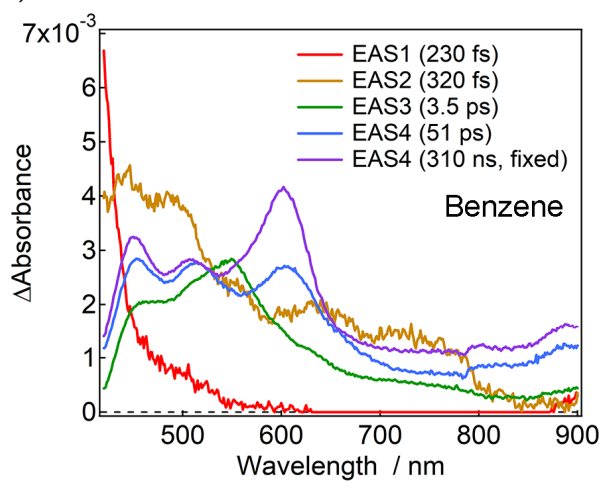

(c)

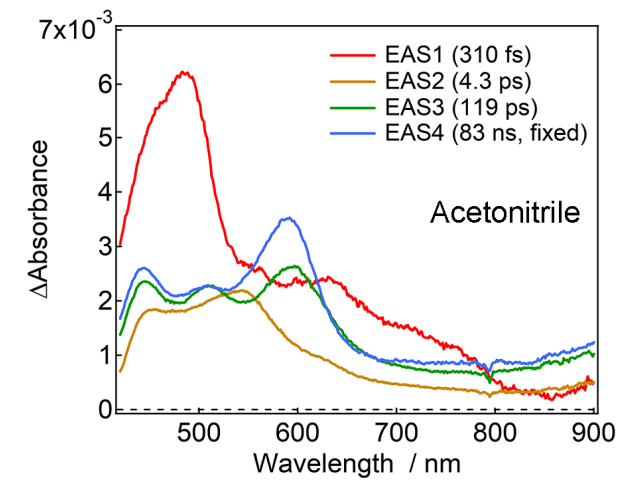

(b)

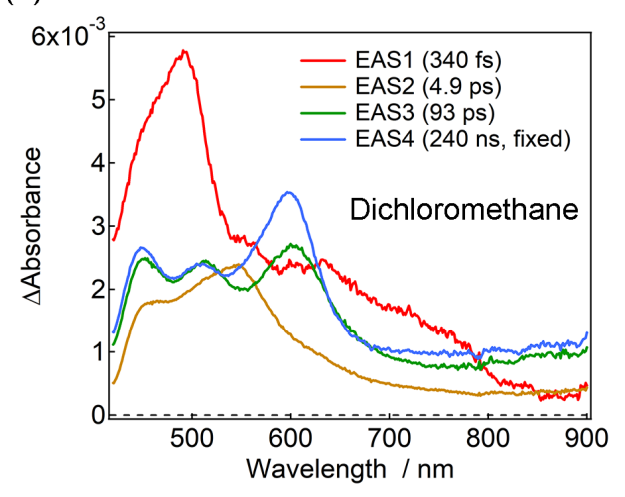

(d)

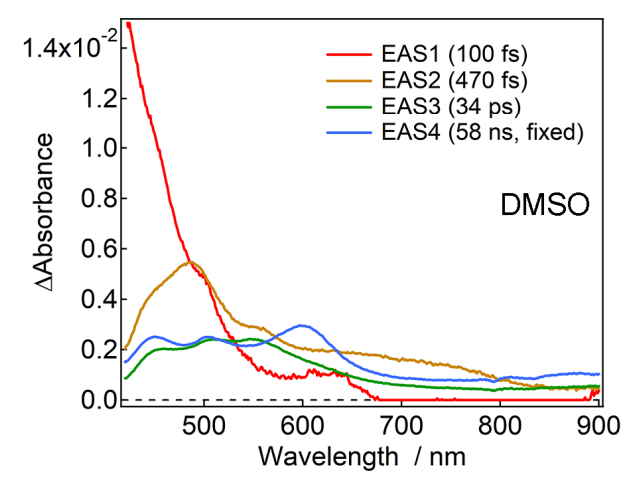

Figure S45. Evolution associated spectra (EAS) of 2 in different solvents excited at $360 \mathrm{~nm}$. EAS1 s in benzene and DMSO are most probably assigned to the $S_{1}$ state of the closed form. On the other hand, the $S_{1}$ state was not observed in dichloromethane and acetonitrile most probably due to the acceleration of the bond cleavage process as was shown in EASs of PIC (Figure S44). Alternatively, EAS1s in dichloromethane and acetonitrile and EAS2 in DMSO show similar spectra. EAS3 in benzene and DMSO, and EAS2 in dichloromethane and acetonitrile would be the charge transfer state of 2 after the bond cleavage.

(a)

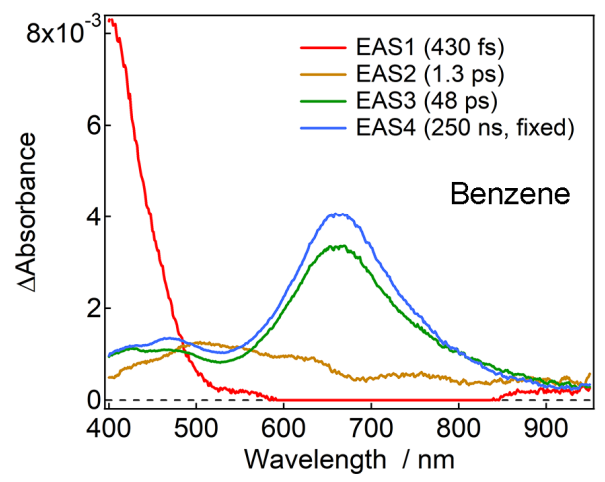

(b)

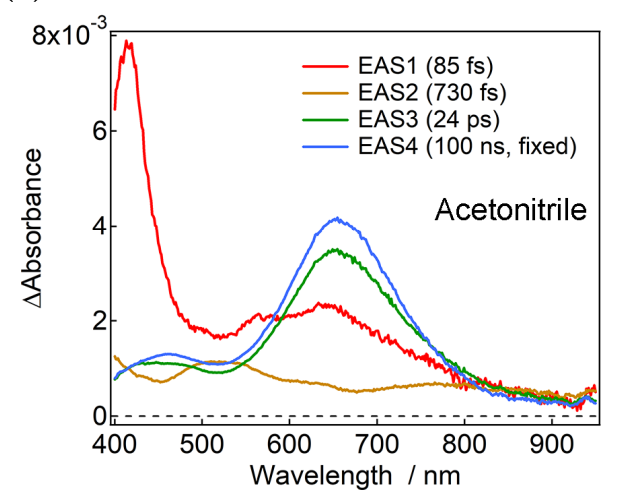

Figure S46. Evolution associated spectra (EAS) of PIC in different solvents excited at $330 \mathrm{~nm}$. 


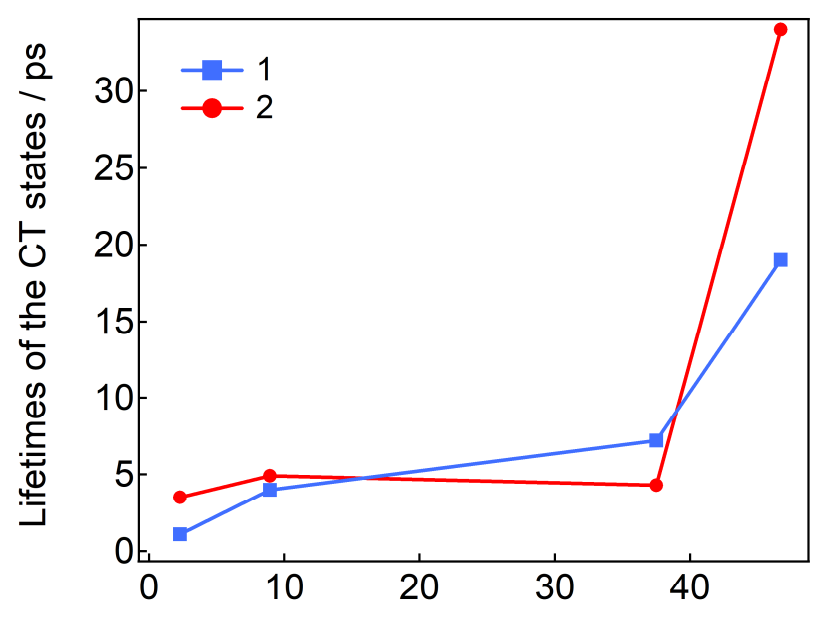

Rerative dielectric constants of the medium

Figure S47. Lifetimes of the CT states of 1 (blue) and 2 (red) generated by the photoinduced bond dissociations.

\section{Picosecond Time-Resolved IR spectra}

(a)

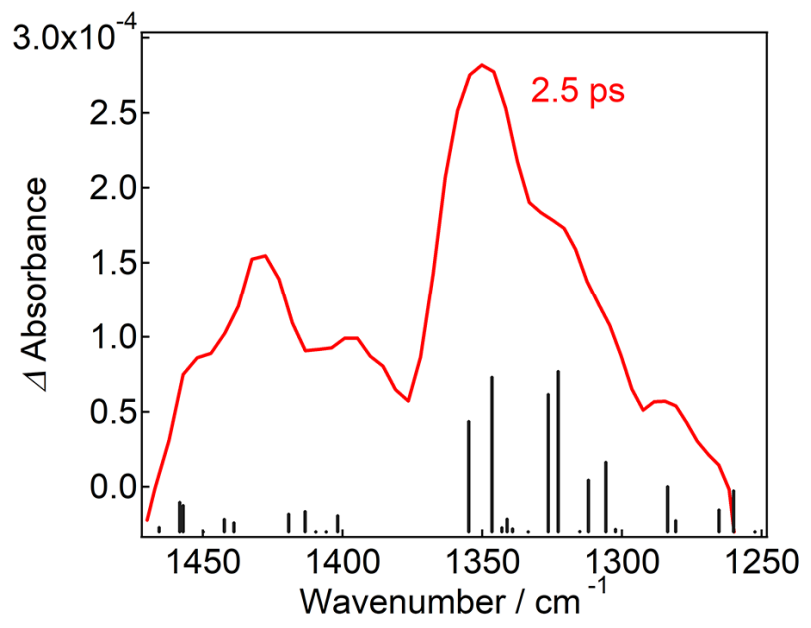

(b)

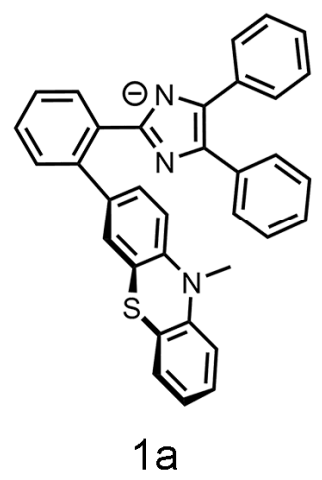

Figure S48. (a) Time-resolved IR spectrum of 1 in $\mathrm{CD}_{2} \mathrm{Cl}_{2}$ at $2.5 \mathrm{ps}$ after the excitation with the $360-\mathrm{nm}$ femtosecond laser pulse and the simulated IR spectrum of the radical anion derivative of 1 (1a) (MPW1PW916$31 \mathrm{G}(\mathrm{d})$ level of theory for $1 \mathrm{a}$, the scale factor is 0.97 ). (b) Molecular structure of $1 \mathrm{a}$. 


\section{Estimation of the Relative Energy Levels of the CT States to the $S_{1}$ states}

The parameters used in the Rehm-Weller equation and the Born equation are shown in Table S3. We used the HOMO-LUMO energy measured by the $\mathrm{CV}$ measurements for $E_{\mathrm{D}^{*}}$. We used the reduction potentials of PABI, PIC, 1 , and 2 for $E_{\text {red }}$ because all reduction reactions produced the imidazole anion. On the other hand, because it was difficult to measure $E_{\mathrm{ox}}$ of the donor moieties of these compounds, we tentatively used the oxidation potentials of non-substituted triphenylimidazole, phenol, phenothiazine, and phenoxazine for $E_{\text {ox }}$. The reduction and oxidation potentials measured in different solvents ( $\Delta_{\text {solv }} E$, acetonitrile and dichloromethane) were corrected for the benzene solution by using the Born equation. $r_{\mathrm{CT}}$ was set to $6 \AA$ based on the optimized structure by DFT calculations. Because the oxidation process of 1 and 2 were irreversible, $E_{\mathrm{D}^{*}}$ of these compounds were roughly estimated from the CV curves.

Table S3. Parameters used for the estimation of the energy levels of the CT states.

\begin{tabular}{lcccc}
\hline & PABI & PIC & 1 & 2 \\
\hline Solvents in CV & acetonitrile & acetonitrile & dichloromethane & dichloromethane \\
measurements & & & & \\
$\varepsilon$ & 36.64 & 36.63 & 8.93 & 8.93 \\
$E_{\mathrm{ox}} / \mathrm{V}(\mathrm{vs} \mathrm{Fc} / \mathrm{Fc}+)^{* 1}$ & $0.947^{\mathrm{S} 1}$ & $0.638^{\mathrm{S} 2}$ & $0.321^{\mathrm{S} 3}$ & $0.441^{\mathrm{S} 4}$ \\
$E_{\mathrm{red}} / \mathrm{V}(\mathrm{vs} \mathrm{Fc} / \mathrm{Fc}+)$ & -1.59 & -2.03 & -1.92 & -1.99 \\
$E_{\mathrm{D}^{*}} / \mathrm{eV}$ & 2.47 & 2.80 & $\sim 2.5$ & $\sim 2.7$ \\
$r_{\mathrm{c}} / \mathrm{pm}$ & $5 \times 10^{2}$ & $3 \times 10^{2}$ & $5 \times 10^{2}$ & $5 \times 10^{2}$ \\
$r_{\mathrm{a}} / \mathrm{pm}$ & $5 \times 10^{2}$ & $5 \times 10^{2}$ & $5 \times 10^{2}$ & $5 \times 10^{2}$ \\
$\Delta G_{\mathrm{CT}} / \mathrm{eV}$ & 0.19 & 0.07 & -0.18 & -0.19 \\
\hline
\end{tabular}

${ }^{*}$ Non-substituted triphenylimidazole, phenol, phenothiazine, and phenoxazine were used for the estimation of $E_{\mathrm{ox}}$ because it is difficult to measure the $E_{\mathrm{ox}}$ of imidazolyl, phenol, phenothiazine, and phenoxazine moieties of above photochromic compounds. To compare the potentials of different reference electrodes, we adjusted to values vs. $\mathrm{Fc} / \mathrm{Fc}^{+}$from the originally reported values (vs. SCE and NHE) by simply subtracting 0.159 and $400 \mathrm{mV}$, respectively. ${ }^{\mathrm{S} 5}$ 


\section{DFT calculations}

All calculations was carried out using the Gaussian 09 program (Revision D.01). ${ }^{\mathrm{S} 6}$ The molecular structure was fully optimized at the M05-2X/6-31G(d) level of theory, and analytical second derivative was computed using vibrational analysis to confirm each stationary point to be a minimum. TDDFT calculations were performed at the MPW1PW91/6-31G+(d) level of the theory for the optimized structures.

Table S4. Standard orientation of the optimized geometry for the closed form of 1.

\begin{tabular}{|c|c|c|c|c|}
\hline \multirow{2}{*}{ Tag } & \multirow{2}{*}{ Symbol } & \multicolumn{3}{|c|}{ Coordinates (Angstroms) } \\
\hline & & $\mathrm{X}$ & Y & Z \\
\hline 1 & $\mathrm{C}$ & -0.7508210 & 5.5991900 & -0.1709690 \\
\hline 2 & $\mathrm{C}$ & 0.5715830 & 5.2969240 & 0.1548130 \\
\hline 3 & $\mathrm{C}$ & 0.9872930 & 3.9644920 & 0.3412940 \\
\hline 4 & $\mathrm{C}$ & 0.0494750 & 2.9588760 & 0.1976710 \\
\hline 5 & $\mathrm{C}$ & -1.3046770 & 3.2705080 & -0.1359270 \\
\hline 6 & $\mathrm{C}$ & -1.7106320 & 4.5822310 & -0.3226800 \\
\hline 7 & $\mathrm{C}$ & -2.0453650 & 2.0180340 & -0.2147300 \\
\hline 8 & $\mathrm{C}$ & 0.2644140 & 1.4372050 & 0.3389460 \\
\hline 9 & $\mathrm{C}$ & 0.6338330 & 1.0755320 & 1.7634770 \\
\hline 10 & $\mathrm{C}$ & 1.7747500 & 0.4642560 & 2.1002700 \\
\hline 11 & $\mathrm{C}$ & 2.7749430 & 0.0757500 & 1.0880860 \\
\hline 12 & $\mathrm{C}$ & 2.3567360 & 0.2992210 & -0.3236010 \\
\hline 13 & $\mathrm{C}$ & 1.2310040 & 0.9334450 & -0.6986810 \\
\hline 14 & $\mathrm{~N}$ & -1.1234750 & 0.9522750 & 0.0336390 \\
\hline 15 & $\mathrm{C}$ & -1.8522880 & -0.2399550 & 0.0435030 \\
\hline 16 & $\mathrm{C}$ & -3.1840170 & 0.1431860 & -0.2447580 \\
\hline 17 & $\mathrm{~N}$ & -3.2849760 & 1.5420460 & -0.3931650 \\
\hline 18 & $\mathrm{~N}$ & 3.9265090 & -0.4382180 & 1.4363450 \\
\hline 19 & $\mathrm{C}$ & 4.8153740 & -0.7999760 & 0.3861520 \\
\hline 20 & $\mathrm{C}$ & 4.4499860 & -0.6598060 & -0.9747500 \\
\hline 21 & $\mathrm{C}$ & 6.0918990 & -1.3218780 & 0.6825370 \\
\hline 22 & $\mathrm{C}$ & 6.9437350 & -1.6846230 & -0.3564720 \\
\hline 23 & $\mathrm{C}$ & 6.5543180 & -1.5394150 & -1.6992360 \\
\hline 24 & $\mathrm{C}$ & 5.3019450 & -1.0230530 & -2.0294840 \\
\hline 25 & $\mathrm{C}$ & -1.2553300 & -1.5392420 & 0.3420180 \\
\hline 26 & $\mathrm{C}$ & -4.3624230 & -0.7087150 & -0.4082270 \\
\hline 27 & $\mathrm{C}$ & -0.2042160 & -2.0415200 & -0.4413500 \\
\hline 28 & $\mathrm{C}$ & 0.3644780 & -3.2792680 & -0.1299510 \\
\hline 29 & $\mathrm{C}$ & -0.1144110 & -4.0204270 & 0.9543520 \\
\hline 30 & $\mathrm{C}$ & -1.1690290 & -3.5255800 & 1.7291310 \\
\hline
\end{tabular}




\begin{tabular}{|c|c|c|c|c|}
\hline 31 & $\mathrm{C}$ & -1.7417320 & -2.2885730 & 1.4277680 \\
\hline 32 & $\mathrm{C}$ & -5.6462590 & -0.1788690 & -0.1744760 \\
\hline 33 & $\mathrm{C}$ & -6.7751250 & -0.9855310 & -0.3213930 \\
\hline 34 & $\mathrm{C}$ & -6.6403870 & -2.3242110 & -0.7047250 \\
\hline 35 & $\mathrm{C}$ & -5.3695750 & -2.8521240 & -0.9478350 \\
\hline 36 & $\mathrm{C}$ & -4.2337190 & -2.0511820 & -0.8049310 \\
\hline 37 & $\mathrm{H}$ & -1.0481600 & 6.6387260 & -0.3118430 \\
\hline 38 & $\mathrm{H}$ & 1.2972710 & 6.1022600 & 0.2653720 \\
\hline 39 & $\mathrm{H}$ & 2.0220520 & 3.7359870 & 0.5900710 \\
\hline 40 & $\mathrm{H}$ & -2.7450780 & 4.8120810 & -0.5785570 \\
\hline 41 & $\mathrm{H}$ & -0.1119540 & 1.3555940 & 2.5114370 \\
\hline 42 & $\mathrm{H}$ & 2.0272690 & 0.2155080 & 3.1358160 \\
\hline 43 & $\mathrm{H}$ & 0.9717980 & 1.1187020 & -1.7425800 \\
\hline 44 & $\mathrm{H}$ & 6.3884110 & -1.4359410 & 1.7275250 \\
\hline 45 & $\mathrm{H}$ & 7.9289890 & -2.0889340 & -0.1271790 \\
\hline 46 & $\mathrm{H}$ & 7.2412450 & -1.8320390 & -2.4940940 \\
\hline 47 & $\mathrm{H}$ & 4.9858920 & -0.8981010 & -3.0647760 \\
\hline 48 & $\mathrm{H}$ & 0.1617890 & -1.4690410 & -1.2939090 \\
\hline 49 & $\mathrm{H}$ & 1.1842400 & -3.6650300 & -0.7342700 \\
\hline 50 & $\mathrm{H}$ & 0.3329740 & -4.9829330 & 1.1959050 \\
\hline 51 & $\mathrm{H}$ & -1.5477850 & -4.1077540 & 2.5679170 \\
\hline 52 & $\mathrm{H}$ & -2.5698490 & -1.9040710 & 2.0238550 \\
\hline 53 & $\mathrm{H}$ & -5.7517060 & 0.8682060 & 0.1164490 \\
\hline 54 & $\mathrm{H}$ & -7.7642890 & -0.5694470 & -0.1390780 \\
\hline 55 & $\mathrm{H}$ & -7.5222140 & -2.9506840 & -0.8160480 \\
\hline 56 & $\mathrm{H}$ & -5.2614840 & -3.8914110 & -1.2523520 \\
\hline 57 & $\mathrm{H}$ & -3.2497240 & -2.4711910 & -1.0111060 \\
\hline 58 & $\mathrm{O}$ & 3.1961690 & -0.1628280 & -1.3476660 \\
\hline
\end{tabular}

SCF Done: $\quad$ E(RM052X)

Zero-point correction

Thermal correction to Energy

Thermal correction to Enthalpy

Thermal correction to Gibbs Free Energy

Sum of electronic and zero-point Energies

Sum of electronic and thermal Energies

Sum of electronic and thermal Enthalpies

Sum of electronic and thermal Free Energies

$\begin{array}{ll}= & -1832.46651733 \quad \text { A.U. } \\ = & 0.458116 \text { (Hartree/Particle) } \\ = & 0.485373 \\ = & 0.486317 \\ = & 0.396991 \\ = & -1832.011222 \\ = & -1831.983965 \\ = & -1831.983021 \\ = & -1832.072346\end{array}$


Low frequencies --- $\begin{array}{lllllll}-9.1863 & -6.1354 & -3.8584 & -0.0002 & 0.0013 & 0.0015\end{array}$

Low frequencies --- $\quad 9.6954 \quad 14.2921 \quad 28.0740$ The Result for the TDDFT calculation

Excited State 1: $\quad$ Singlet-A $\quad 2.6700 \mathrm{eV} \quad 464.35 \mathrm{~nm} \quad \mathrm{f}=0.0005 \quad<\mathrm{S}^{* * 2}>=0.000$

$$
128->129 \quad 0.70310
$$

This state for optimization and/or second-order correction.

Total Energy, E(TD-HF/TD-KS) $=-1832.22404045$

Copying the excited state density for this state as the 1-particle RhoCI density.

\begin{tabular}{|c|c|c|c|c|c|c|}
\hline Excited State & 2: & Singlet-A & $3.0542 \mathrm{eV}$ & $405.95 \mathrm{~nm}$ & $\mathrm{f}=0.1481$ & $<\mathrm{S}^{* * 2} 2>=0.000$ \\
\hline $127->129$ & & 0.69252 & & & & \\
\hline Excited State & 3: & Singlet-A & $3.8618 \mathrm{eV}$ & $321.05 \mathrm{~nm}$ & $\mathrm{f}=0.1817$ & $<\mathrm{S} * * 2>=0.000$ \\
\hline $128->130$ & & 0.67969 & & & & \\
\hline $128->131$ & & -0.12880 & & & & \\
\hline Excited State & 4: & Singlet-A & $3.9307 \mathrm{eV}$ & $315.43 \mathrm{~nm}$ & $\mathrm{f}=0.0842$ & $<\mathrm{S} * * 2>=0.000$ \\
\hline $120->129$ & & 0.52237 & & & & \\
\hline $124->129$ & & -0.40250 & & & & \\
\hline $125->129$ & & -0.11415 & & & & \\
\hline $126->129$ & & -0.11967 & & & & \\
\hline
\end{tabular}

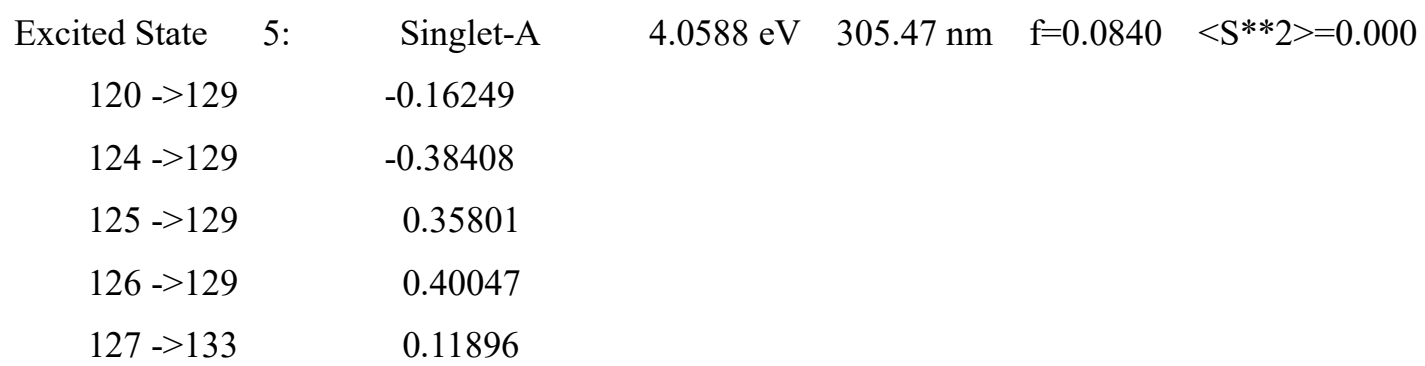

\begin{tabular}{|c|c|c|c|c|}
\hline Excited State & 6: & Singlet-A & $4.0818 \mathrm{eV}$ & $303.75 \mathrm{~nm} \quad \mathrm{f}=0.1101$ \\
\hline $119->129$ & & 0.11270 & & \\
\hline $120->129$ & & 0.40122 & & \\
\hline $124->129$ & & 0.34250 & & \\
\hline $125->129$ & & 0.23370 & & \\
\hline $126->129$ & & 0.32230 & & \\
\hline $127->133$ & & -0.12692 & & \\
\hline
\end{tabular}




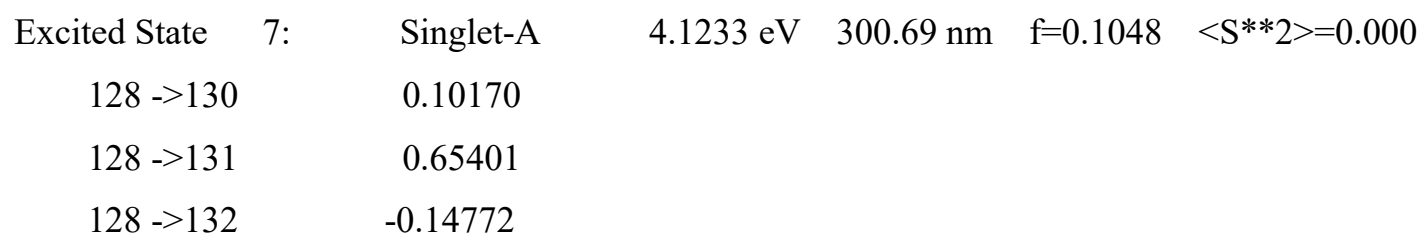
Excited State $\quad 8: \quad$ Singlet-A $\quad 4.1320 \mathrm{eV} \quad 300.06 \mathrm{~nm} \quad \mathrm{f}=0.3002 \quad<\mathrm{S}^{* *} 2>=0.000$ $128->130 \quad 0.10925$
$128->131 \quad 0.12694$
$128->132 \quad 0.66018$

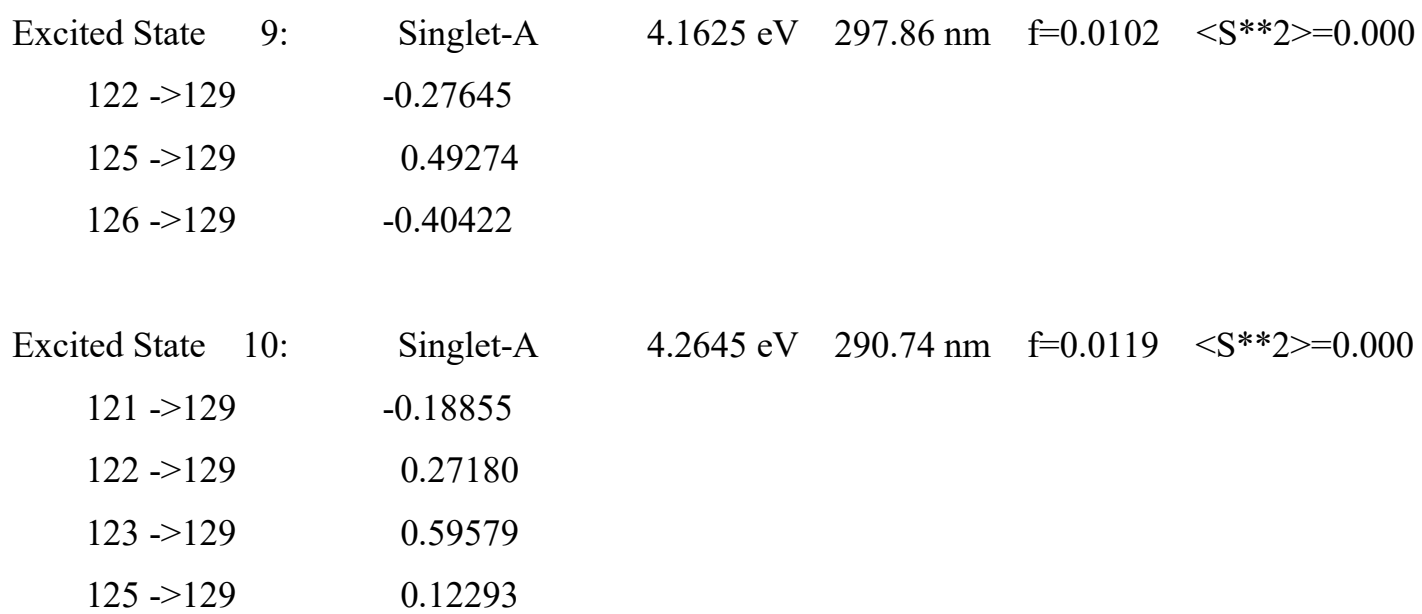

Excited State 11: $\quad$ Singlet-A $\quad 4.3318 \mathrm{eV} \quad 286.22 \mathrm{~nm} \quad \mathrm{f}=0.0123 \quad<\mathrm{S} * * 2>=0.000$

$\begin{array}{cc}121->129 & -0.13010 \\ 122->129 & 0.44386 \\ 123->129 & -0.28154 \\ 125->129 & 0.12428 \\ 126->129 & -0.18354 \\ 127->130 & -0.35504 \\ 127->131 & 0.10027\end{array}$

Excited State 12: $\quad$ Singlet-A $\quad 4.3465 \mathrm{eV} \quad 285.25 \mathrm{~nm} \quad \mathrm{f}=0.0498 \quad<\mathrm{S}^{* *} 2>=0.000$

$\begin{array}{rr}122->129 & 0.29464 \\ 123->129 & -0.19088 \\ 126->129 & -0.10593 \\ 127->130 & 0.56755\end{array}$

Excited State 13: $\quad$ Singlet-A $\quad 4.4172 \mathrm{eV} \quad 280.69 \mathrm{~nm} \quad \mathrm{f}=0.0310 \quad<\mathrm{S} * * 2>=0.000$

$121->129 \quad 0.40016$

$122->129 \quad 0.12650$ 


\begin{tabular}{|c|c|c|c|c|c|}
\hline $128->134$ & 0.46317 & & & & \\
\hline $128->135$ & 0.22947 & & & & \\
\hline Excited State 14: & Singlet-A & $4.4283 \mathrm{eV}$ & $279.98 \mathrm{~nm}$ & $\mathrm{f}=0.0063$ & $<\mathrm{S} * * 2>=0.000$ \\
\hline $121->129$ & 0.45926 & & & & \\
\hline $122->129$ & 0.19917 & & & & \\
\hline $127->133$ & -0.11758 & & & & \\
\hline $128->134$ & -0.38853 & & & & \\
\hline $128->135$ & -0.21055 & & & & \\
\hline Excited State 15: & Singlet-A & $4.5526 \mathrm{eV}$ & $272.33 \mathrm{~nm}$ & $\mathrm{f}=0.1188$ & $<\mathrm{S} * * 2>=0.000$ \\
\hline $127->130$ & 0.13958 & & & & \\
\hline $127->131$ & 0.57972 & & & & \\
\hline $127->132$ & -0.26063 & & & & \\
\hline $127->134$ & -0.11777 & & & & \\
\hline $127->135$ & 0.11435 & & & & \\
\hline Excited State 16 : & Singlet-A & $4.5853 \mathrm{eV}$ & $270.40 \mathrm{~nm}$ & $\mathrm{f}=0.0041$ & $<\mathrm{S} * * 2>=0.000$ \\
\hline $128->132$ & -0.10556 & & & & \\
\hline $128->133$ & 0.39867 & & & & \\
\hline $128->134$ & -0.21854 & & & & \\
\hline $128->135$ & 0.48817 & & & & \\
\hline Excited State $17:$ & Singlet-A & $4.6368 \mathrm{eV}$ & $267.39 \mathrm{~nm}$ & $\mathrm{f}=0.0054$ & $<\mathrm{S} * * 2>=0.000$ \\
\hline $128->133$ & 0.56957 & & & & \\
\hline $128->134$ & 0.19910 & & & & \\
\hline $128->135$ & -0.31826 & & & & \\
\hline Excited State $18:$ & Singlet-A & $4.7098 \mathrm{eV}$ & $263.25 \mathrm{~nm}$ & $\mathrm{f}=0.0366$ & $<\mathrm{S} * * 2>=0.000$ \\
\hline $127->131$ & 0.30951 & & & & \\
\hline $127->132$ & 0.57787 & & & & \\
\hline $127->135$ & -0.11196 & & & & \\
\hline Excited State $19:$ & Singlet-A & $4.7382 \mathrm{eV}$ & $261.67 \mathrm{~nm}$ & $\mathrm{f}=0.2227$ & $<\mathrm{S} * * 2>=0.000$ \\
\hline $118->129$ & -0.21526 & & & & \\
\hline $119->129$ & -0.25815 & & & & \\
\hline $121->129$ & 0.22091 & & & & \\
\hline $124->129$ & 0.13457 & & & & \\
\hline $127->133$ & 0.47995 & & & & \\
\hline
\end{tabular}


$128->136-0.10212$

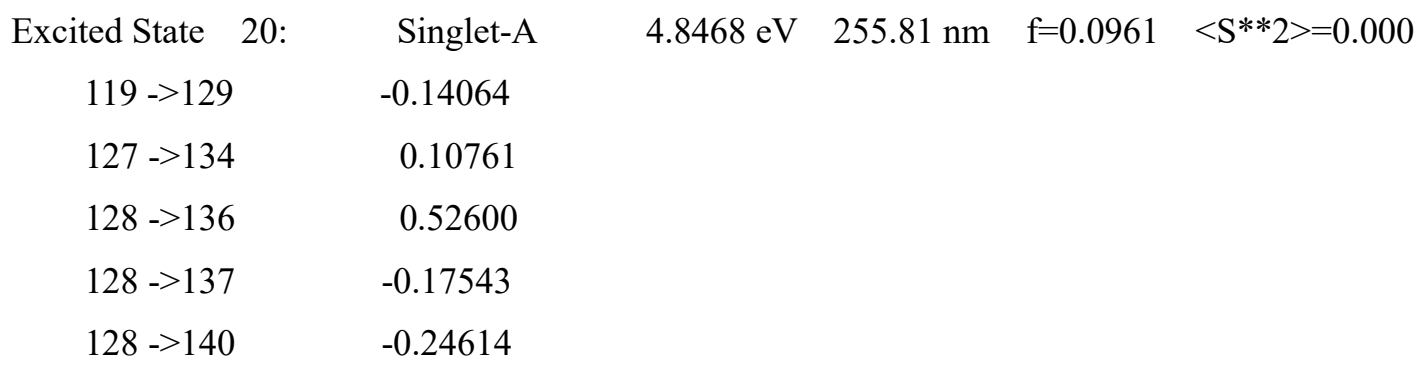

Excited State 21: $\quad$ Singlet-A $\quad 4.8791 \mathrm{eV} \quad 254.11 \mathrm{~nm} \quad \mathrm{f}=0.0522<\mathrm{S} * * 2>=0.000$ $118->129-0.11858$

$119->129 \quad-0.22163$

$126->132-0.11481$

$127->132 \quad-0.18403$

$127->133 \quad-0.22741$

$127->134 \quad 0.26578$

$127->135 \quad-0.17613$

$127->137 \quad 0.26197$

$127->138 \quad 0.14055$

$127->143 \quad 0.10816$

$128->139 \quad 0.14179$

$128->140 \quad 0.23250$

$\begin{array}{cc}\text { Excited State } 22: & \text { Singlet-A } \\ 118->129 & 0.14888 \\ 119->129 & 0.34771 \\ 126->132 & -0.16294 \\ 127->133 & 0.22065 \\ 127->134 & 0.12452 \\ 127->135 & -0.13232 \\ 128->136 & 0.28871 \\ 128->139 & 0.20220 \\ 128->140 & 0.24201\end{array}$

\begin{tabular}{|c|c|c|c|}
\hline Excited State 23: & Singlet-A & $4.9152 \mathrm{eV}$ & $252.25 \mathrm{~nm} \quad \mathrm{f}=0.0452$ \\
\hline $118->129$ & 0.10604 & & \\
\hline $119->129$ & 0.30057 & & \\
\hline $126->132$ & 0.12954 & & \\
\hline $127->132$ & -0.16186 & & \\
\hline
\end{tabular}




$\begin{array}{lr}127->133 & 0.11349 \\ 127->134 & 0.24262 \\ 127->135 & -0.16710 \\ 127->137 & 0.14247 \\ 128->136 & -0.24571 \\ 128->137 & -0.18776 \\ 128->139 & -0.16567 \\ 128->140 & -0.22567\end{array}$

Excited State 24: Singlet-A $\quad 4.9803 \mathrm{eV} \quad 248.95 \mathrm{~nm} \quad \mathrm{f}=0.0006 \quad<\mathrm{S} * * 2>=0.000$

$\begin{array}{lr}126->132 & 0.11472 \\ 127->134 & 0.11356 \\ 128->137 & 0.60347 \\ 128->138 & -0.12066 \\ 128->139 & -0.10003 \\ 128->140 & -0.14832 \\ 128->144 & 0.11461\end{array}$

Excited State 25: $\quad$ Singlet-A $\quad 5.0786 \mathrm{eV} \quad 244.13 \mathrm{~nm} \quad \mathrm{f}=0.0058 \quad<\mathrm{S}^{* *} 2>=0.000$

$\begin{array}{lr}127->134 & 0.51218 \\ 127->135 & 0.37920 \\ 127->137 & -0.19230 \\ 127->138 & -0.10498\end{array}$

Excited State 26: $\quad$ Singlet-A $\quad 5.1061 \mathrm{eV} \quad 242.82 \mathrm{~nm} \quad \mathrm{f}=0.0051 \quad<\mathrm{S}^{* *} 2>=0.000$

$\begin{array}{cc}116->129 & 0.11319 \\ 118->129 & 0.55431 \\ 119->129 & -0.32358 \\ 125->130 & 0.11998 \\ 126->130 & 0.10152 \\ 127->135 & -0.10781\end{array}$

Excited State $\quad 27: \quad$ Singlet-A $\quad 5.1614 \mathrm{eV} \quad 240.21 \mathrm{~nm} \quad \mathrm{f}=0.0608 \quad<\mathrm{S} * * 2>=0.000$

$\begin{array}{ll}116->129 & 0.14015 \\ 125->130 & -0.10666 \\ 126->130 & -0.15110 \\ 127->135 & -0.31860 \\ 127->137 & -0.21914 \\ 127->138 & -0.14177\end{array}$




$\begin{array}{rr}128->138 & 0.34068 \\ 128->139 & -0.21597 \\ 128->140 & 0.13242\end{array}$

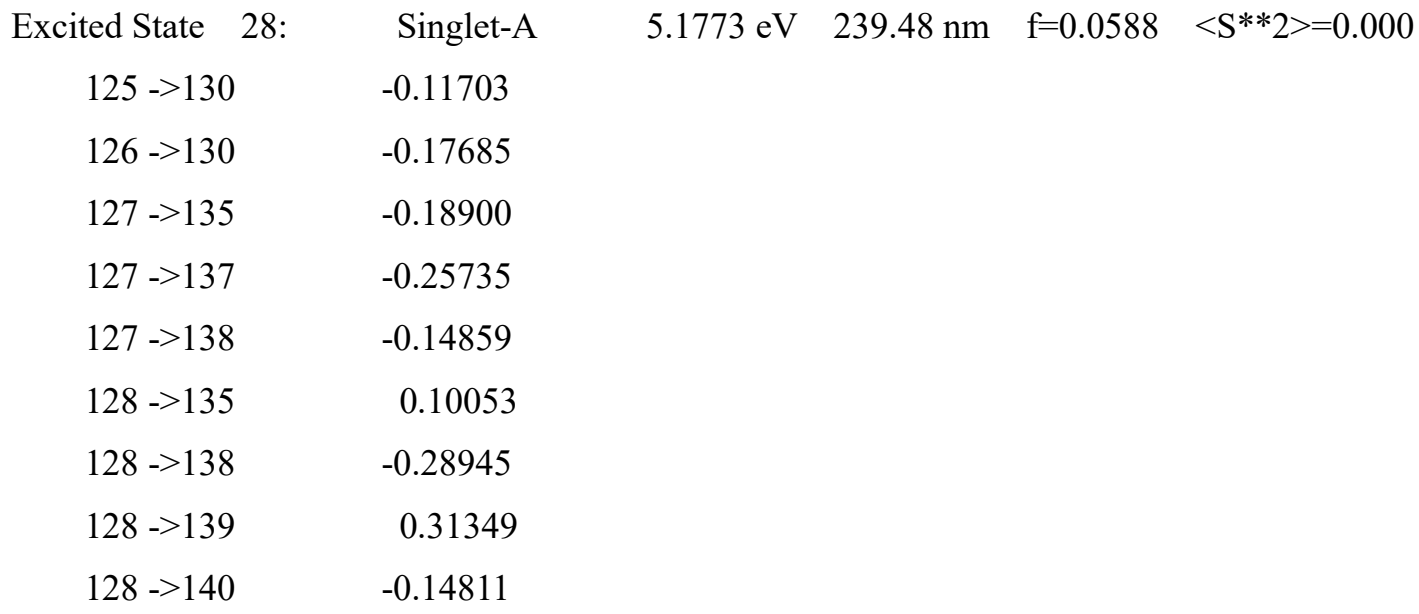

Excited State 29: $\quad$ Singlet-A $\quad 5.2031 \mathrm{eV} \quad 238.29 \mathrm{~nm} \quad \mathrm{f}=0.1069 \quad<\mathrm{S}^{* *} 2>=0.000$

$\begin{array}{lc}118->129 & -0.18434 \\ 125->130 & 0.38641 \\ 126->130 & 0.43586 \\ 127->135 & -0.16172 \\ 127->137 & -0.10348\end{array}$

Excited State $\quad 30: \quad$ Singlet-A $\quad 5.2469 \mathrm{eV} \quad 236.30 \mathrm{~nm} \quad \mathrm{f}=0.0785 \quad<\mathrm{S} * * 2>=0.000$

$\begin{array}{lr}121->130 & -0.24095 \\ 123->131 & 0.13889 \\ 124->130 & 0.48453 \\ 125->130 & -0.11847 \\ 125->131 & 0.20050 \\ 125->132 & 0.10559 \\ 126->131 & 0.12450 \\ 128->138 & 0.15205\end{array}$

Excited State $\quad 31: \quad$ Singlet-A $\quad 5.2759 \mathrm{eV} \quad 235.00 \mathrm{~nm} \quad \mathrm{f}=0.0014 \quad<\mathrm{S} * * 2>=0.000$

$\begin{array}{lr}125->130 & 0.43866 \\ 125->132 & -0.15482 \\ 126->130 & -0.43133 \\ 128->138 & 0.11920\end{array}$

Excited State $\quad 32: \quad$ Singlet-A $\quad 5.2962 \mathrm{eV} \quad 234.10 \mathrm{~nm} \quad \mathrm{f}=0.0420 \quad<\mathrm{S} * * 2>=0.000$ 


$\begin{array}{rr}116->129 & -0.22357 \\ 117->129 & -0.16132 \\ 124->130 & -0.10343 \\ 128->138 & 0.38930 \\ 128->139 & 0.35473 \\ 128->140 & -0.17829\end{array}$

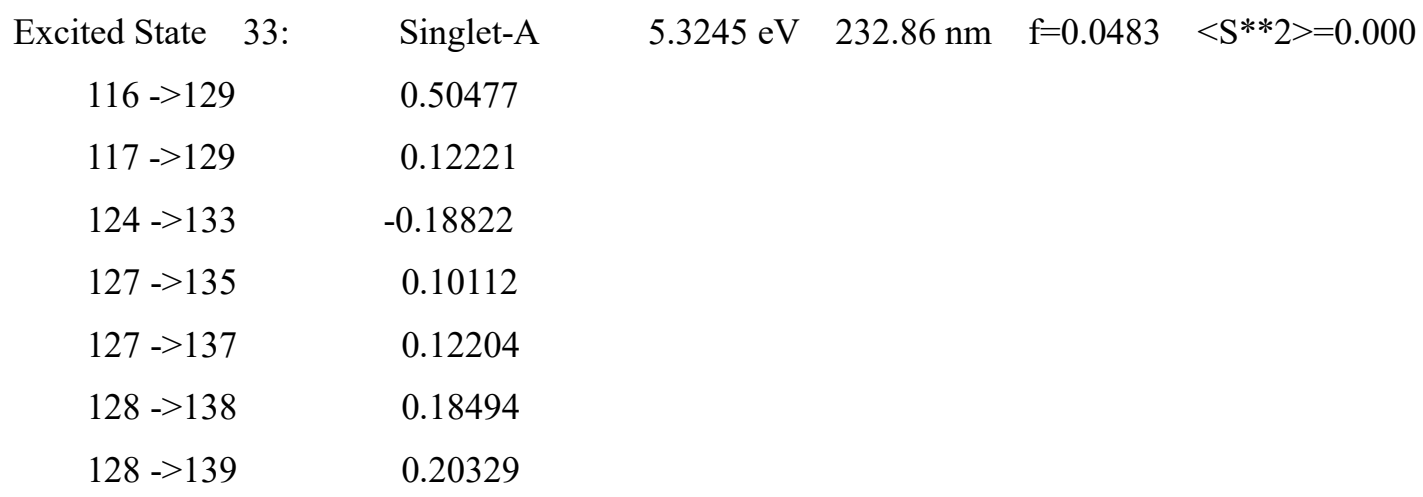

Excited State $\quad 34: \quad$ Singlet-A $\quad 5.3588 \mathrm{eV} \quad 231.36 \mathrm{~nm} \quad \mathrm{f}=0.0068 \quad<\mathrm{S} * * 2>=0.000$

$\begin{array}{cc}117->129 & -0.25566 \\ 127->136 & -0.20852 \\ 127->137 & -0.14655 \\ 127->138 & 0.35495 \\ 128->140 & -0.11020 \\ 128->141 & 0.31569 \\ 128->142 & 0.22264\end{array}$

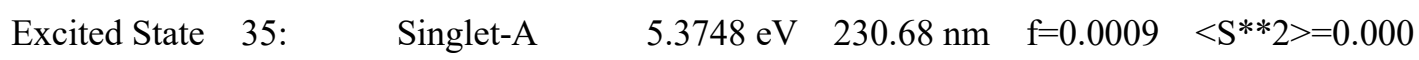

$\begin{array}{lr}115->129 & 0.10147 \\ 116->129 & 0.11567 \\ 117->129 & -0.16895 \\ 126->132 & -0.12072 \\ 127->136 & 0.29612 \\ 127->137 & 0.14333 \\ 127->138 & -0.28665 \\ 127->139 & -0.15198 \\ 128->140 & -0.10094 \\ 128->141 & 0.36642\end{array}$

Excited State $36: \quad$ Singlet-A $\quad 5.3818 \mathrm{eV} \quad 230.38 \mathrm{~nm} \quad \mathrm{f}=0.0032 \quad<\mathrm{S} * * 2>=0.000$ $116->129 \quad-0.12939$ 


$\begin{array}{rr}117->129 & 0.58451 \\ 128->139 & 0.11165 \\ 128->140 & -0.11367 \\ 128->141 & 0.22905 \\ 128->142 & 0.15188\end{array}$

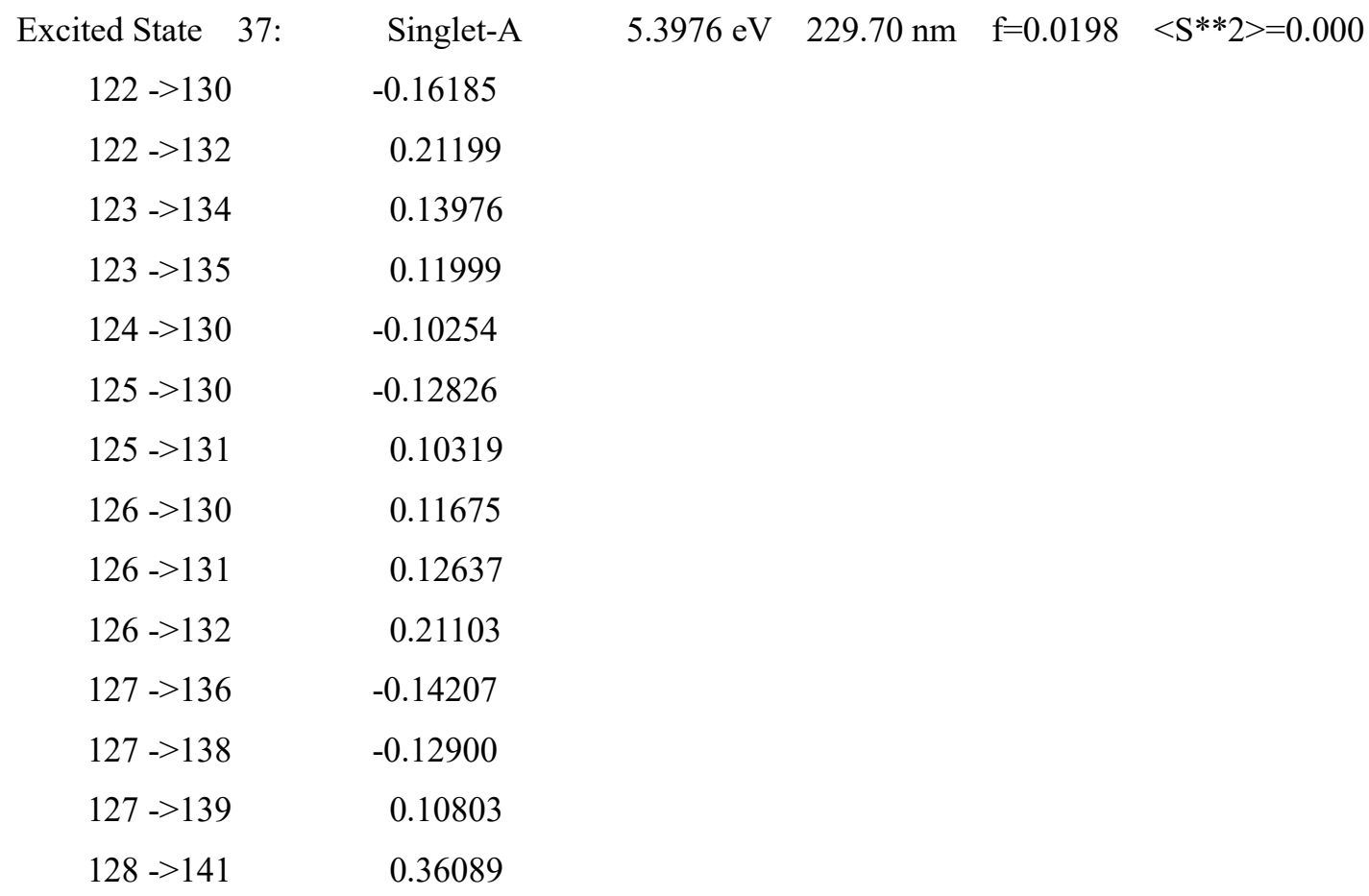

Excited State $\quad 38: \quad$ Singlet-A $\quad 5.4054 \mathrm{eV} \quad 229.37 \mathrm{~nm} \quad \mathrm{f}=0.0397 \quad<\mathrm{S} * * 2>=0.000$

$\begin{array}{lr}121->130 & -0.10945 \\ 123->130 & -0.21696 \\ 125->131 & 0.14333 \\ 126->131 & 0.12606 \\ 127->136 & 0.11453 \\ 128->138 & -0.11687 \\ 128->141 & -0.14405 \\ 128->142 & 0.48060 \\ 128->144 & -0.11824 \\ 128->145 & 0.11261\end{array}$

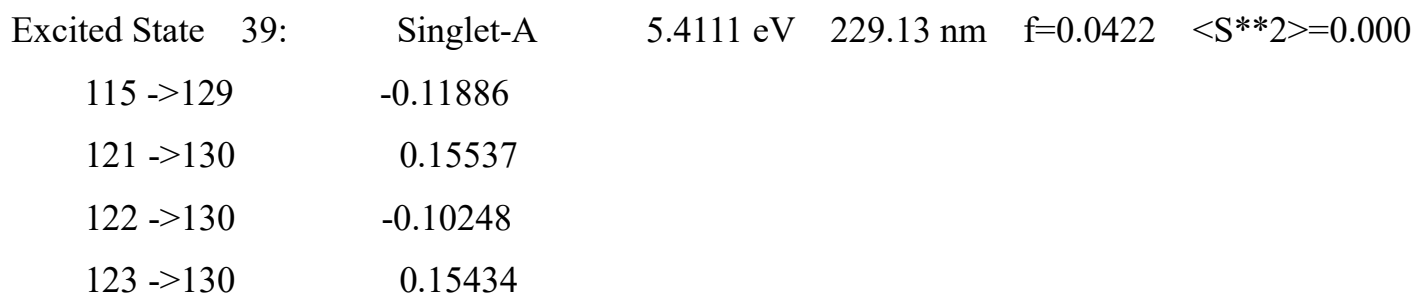




$\begin{array}{lc}124->130 & 0.23076 \\ 124->131 & -0.18018 \\ 125->131 & -0.17614 \\ 125->132 & -0.16003 \\ 126->131 & -0.11227 \\ 127->137 & 0.10144 \\ 127->138 & -0.21867 \\ 127->139 & 0.14298 \\ 128->142 & 0.27724\end{array}$

Excited State $\quad 40: \quad$ Singlet-A $\quad 5.4339 \mathrm{eV} \quad 228.17 \mathrm{~nm} \quad \mathrm{f}=0.0119 \quad<\mathrm{S} * * 2>=0.000$

$\begin{array}{lr}118->130 & -0.10626 \\ 119->130 & 0.13595 \\ 122->130 & -0.14936 \\ 122->132 & 0.13876 \\ 123->130 & 0.41086 \\ 125->132 & -0.20552 \\ 127->136 & 0.14536 \\ 127->138 & 0.21242 \\ 127->139 & -0.18237 \\ 127->140 & 0.12627\end{array}$

Table S5. Standard orientation of the optimized geometry for the singlet biradical form of the ring-opening form of 1 .

\begin{tabular}{ccccc}
\hline Tag & Symbol & \multicolumn{3}{c}{ Coordinates (Angstroms) } \\
& & $\mathrm{X}$ & $\mathrm{Y}$ & $\mathrm{Z}$ \\
\hline 1 & $\mathrm{C}$ & -2.3223180 & 5.2294840 & -0.1753470 \\
2 & $\mathrm{C}$ & -0.2149300 & 3.4165640 & 0.2792450 \\
3 & $\mathrm{C}$ & -1.0452910 & 5.6993030 & 0.1203390 \\
4 & $\mathrm{C}$ & -2.5540940 & 3.8677990 & -0.2169520 \\
5 & $\mathrm{C}$ & -1.5268240 & 2.9405960 & 0.0345090 \\
6 & $\mathrm{C}$ & -0.0125710 & 4.7984750 & 0.3375020 \\
7 & $\mathrm{C}$ & 0.9811070 & 2.5508540 & 0.4131330 \\
8 & $\mathrm{C}$ & 3.3581980 & 1.0077570 & 0.6151280 \\
9 & $\mathrm{C}$ & 1.3114750 & 1.6228430 & -0.5713570 \\
10 & $\mathrm{C}$ & 1.8605560 & 2.7181700 & 1.4988350 \\
11 & $\mathrm{C}$ & 3.0044610 & 1.9649540 & 1.5989630 \\
12 & $\mathrm{C}$ & 2.4693190 & 0.8610120 & -0.4851260
\end{tabular}




\begin{tabular}{|c|c|c|c|c|}
\hline 13 & $\mathrm{~S}$ & 2.7883950 & -0.2826460 & -1.7778440 \\
\hline 14 & $\mathrm{C}$ & 4.3130480 & -0.9874910 & -1.2730180 \\
\hline 15 & $\mathrm{C}$ & 4.9549990 & -0.5998640 & -0.0700340 \\
\hline 16 & $\mathrm{~N}$ & 4.5103390 & 0.3301590 & 0.8159780 \\
\hline 17 & $\mathrm{C}$ & 6.1795700 & -1.2356360 & 0.2484700 \\
\hline 18 & $\mathrm{C}$ & 6.7330850 & -2.1925970 & -0.5711290 \\
\hline 19 & $\mathrm{C}$ & 6.0809800 & -2.5585730 & -1.7550770 \\
\hline 20 & $\mathrm{C}$ & 4.8815730 & -1.9581410 & -2.0985700 \\
\hline 21 & $\mathrm{C}$ & -1.9124310 & 1.5411550 & 0.1044710 \\
\hline 22 & $\mathrm{~N}$ & -3.1104980 & 1.1123920 & -0.3976420 \\
\hline 23 & $\mathrm{C}$ & -3.1378070 & -0.1820900 & -0.1382050 \\
\hline 24 & $\mathrm{C}$ & -1.9063500 & -0.5240020 & 0.6083900 \\
\hline 25 & $\mathrm{~N}$ & -1.1755010 & 0.5731860 & 0.7123510 \\
\hline 26 & $\mathrm{C}$ & -4.2312410 & -1.0253050 & -0.6219670 \\
\hline 27 & $\mathrm{C}$ & -6.3479640 & -2.5445850 & -1.6403120 \\
\hline 28 & $\mathrm{C}$ & -4.0350990 & -2.3620930 & -0.9902100 \\
\hline 29 & $\mathrm{C}$ & -5.5010580 & -0.4565480 & -0.7911690 \\
\hline 30 & $\mathrm{C}$ & -6.5508020 & -1.2127020 & -1.2874650 \\
\hline 31 & $\mathrm{C}$ & -5.0871640 & -3.1132540 & -1.4970040 \\
\hline 32 & $\mathrm{C}$ & -1.4759210 & -1.7712420 & 1.2374180 \\
\hline 33 & $\mathrm{C}$ & -0.5645080 & -4.0844330 & 2.5184910 \\
\hline 34 & $\mathrm{C}$ & -0.1028880 & -1.9918550 & 1.4199030 \\
\hline 35 & $\mathrm{C}$ & -2.3844780 & -2.7188250 & 1.7262850 \\
\hline 36 & $\mathrm{C}$ & -1.9290580 & -3.8652860 & 2.3630210 \\
\hline 37 & $\mathrm{C}$ & 0.3467540 & -3.1426500 & 2.0469380 \\
\hline 38 & $\mathrm{H}$ & -3.1340710 & 5.9247190 & -0.3635910 \\
\hline 39 & $\mathrm{H}$ & -0.8483250 & 6.7660330 & 0.1619990 \\
\hline 40 & $\mathrm{H}$ & -3.5433130 & 3.4776210 & -0.4237030 \\
\hline 41 & $\mathrm{H}$ & 0.9920390 & 5.1669680 & 0.5192570 \\
\hline 42 & $\mathrm{H}$ & 0.6519340 & 1.4950860 & -1.4241620 \\
\hline 43 & $\mathrm{H}$ & 1.6131830 & 3.4346060 & 2.2755030 \\
\hline 44 & $\mathrm{H}$ & 3.6830270 & 2.0713070 & 2.4379220 \\
\hline 45 & $\mathrm{H}$ & 6.6581010 & -0.9276740 & 1.1715270 \\
\hline 46 & $\mathrm{H}$ & 7.6733870 & -2.6622050 & -0.3009550 \\
\hline 47 & $\mathrm{H}$ & 6.5112430 & -3.3117390 & -2.4070250 \\
\hline 48 & $\mathrm{H}$ & 4.3745470 & -2.2407880 & -3.0167030 \\
\hline 49 & $\mathrm{H}$ & -7.1701600 & -3.1353660 & -2.0322130 \\
\hline 50 & $\mathrm{H}$ & -3.0512670 & -2.8076470 & -0.8987660 \\
\hline 51 & $\mathrm{H}$ & -5.6412360 & 0.5851940 & -0.5263550 \\
\hline
\end{tabular}




\begin{tabular}{rlrrr}
52 & $\mathrm{H}$ & -7.5317650 & -0.7625610 & -1.4036600 \\
53 & $\mathrm{H}$ & -4.9186910 & -4.1456390 & -1.7869150 \\
54 & $\mathrm{H}$ & -0.2109410 & -4.9841480 & 3.0126760 \\
55 & $\mathrm{H}$ & 0.5951000 & -1.2434210 & 1.0618730 \\
56 & $\mathrm{H}$ & -3.4499150 & -2.5465990 & 1.6276640 \\
57 & $\mathrm{H}$ & -2.6441290 & -4.5868690 & 2.7455480 \\
58 & $\mathrm{H}$ & 1.4125740 & -3.3054860 & 2.1721780 \\
\hline
\end{tabular}

SCF Done: $\quad$ E(UCAM-B3LYP)

$=-1831.76631978 \quad$ A.U.

Zero-point correction

$=0.452596($ Hartree/Particle $)$

Thermal correction to Energy

$=0.480377$

Thermal correction to Enthalpy

$=0.481321$

Thermal correction to Gibbs Free Energy

$=0.390528$

Sum of electronic and zero-point Energies

$=-1831.314026$

Sum of electronic and thermal Energies

$=-1831.286245$

Sum of electronic and thermal Enthalpies

$=-1831.285301$

Sum of electronic and thermal Free Energies

$=-1831.376094$

$\begin{array}{lllllll}\text { Low frequencies --- } & -0.9416 & -0.0013 & -0.0011 & 0.0005 & 0.7195 & 1.2952\end{array}$

$\begin{array}{llll}\text { Low frequencies --- } & 9.7584 & 15.2707 & 21.7982\end{array}$

The Results of the TDDFT calculation

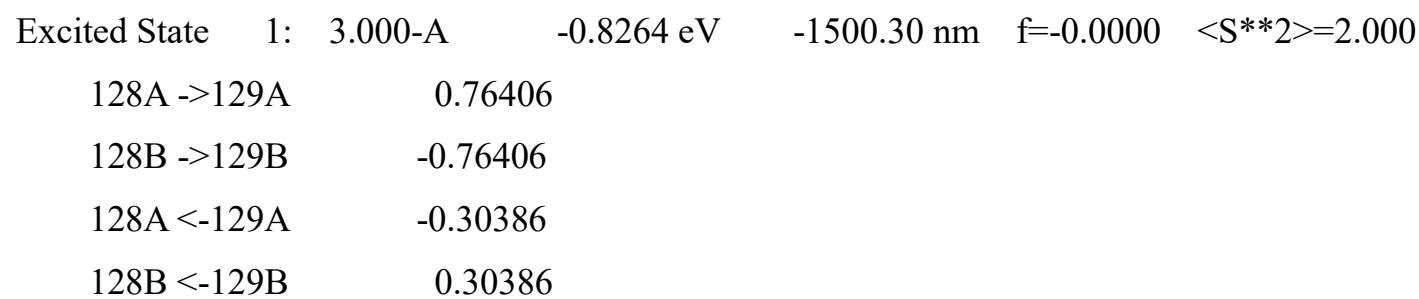

This state for optimization and/or second-order correction.

Total Energy, E(TD-HF/TD-DFT) $=-1831.81231703$

Copying the excited state density for this state as the 1-particle RhoCI density.

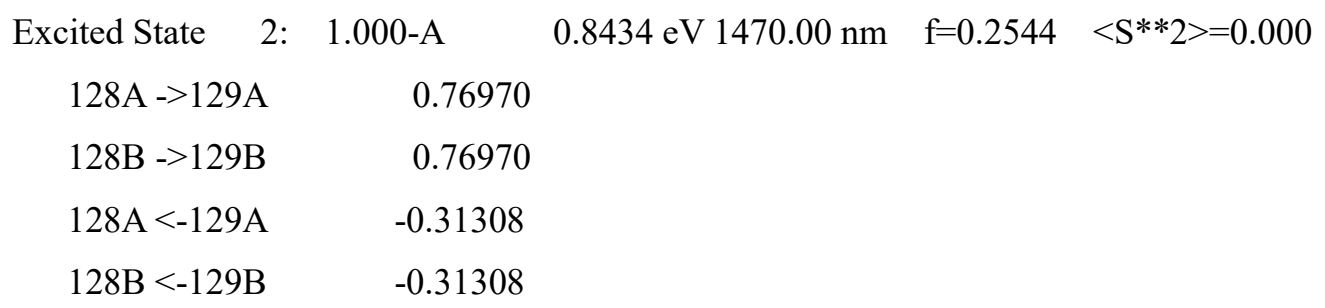

Excited State $\quad 3: \quad 3.000-\mathrm{A} \quad 1.5351 \mathrm{eV} \quad 807.68 \mathrm{~nm} \quad \mathrm{f}=0.0000 \quad<\mathrm{S}^{* *} 2>=2.000$ 


$\begin{array}{lr}118 \mathrm{~A}->129 \mathrm{~A} & -0.10680 \\ 122 \mathrm{~A}->129 \mathrm{~A} & 0.15673 \\ 124 \mathrm{~A}->129 \mathrm{~A} & -0.20895 \\ 125 \mathrm{~A}->129 \mathrm{~A} & 0.22559 \\ 126 \mathrm{~A}->129 \mathrm{~A} & 0.40784 \\ 127 \mathrm{~A}->129 \mathrm{~A} & 0.43584 \\ 118 \mathrm{~B}->129 \mathrm{~B} & 0.10680 \\ 122 \mathrm{~B}->129 \mathrm{~B} & -0.15673 \\ 124 \mathrm{~B}->129 \mathrm{~B} & 0.20895 \\ 125 \mathrm{~B}->129 \mathrm{~B} & -0.22559 \\ 126 \mathrm{~B}->129 \mathrm{~B} & -0.40784 \\ 127 \mathrm{~B}->129 \mathrm{~B} & -0.43584\end{array}$

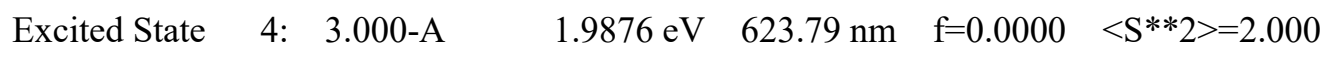
$118 \mathrm{~A}->129 \mathrm{~A}$
0.14037
$121 \mathrm{~A}->129 \mathrm{~A}$
0.10328
$122 \mathrm{~A}->129 \mathrm{~A}$
0.44701
$124 \mathrm{~A}->129 \mathrm{~A}$
$-0.15965$
$125 \mathrm{~A} \rightarrow 129 \mathrm{~A}$
0.19474
$127 \mathrm{~A} \rightarrow 129 \mathrm{~A}$
$-0.38033$
$128 \mathrm{~A}->130 \mathrm{~A}$
$-0.11057$
$118 \mathrm{~B}->129 \mathrm{~B}$
$-0.14037$
$121 \mathrm{~B}->129 \mathrm{~B}$
$-0.10328$
$122 \mathrm{~B}->129 \mathrm{~B}$
$-0.44701$
$124 \mathrm{~B}->129 \mathrm{~B}$
0.15965
$125 \mathrm{~B}->129 \mathrm{~B}$
$-0.19474$
$127 \mathrm{~B}->129 \mathrm{~B}$
0.38033
$128 \mathrm{~B}->130 \mathrm{~B}$
0.11057

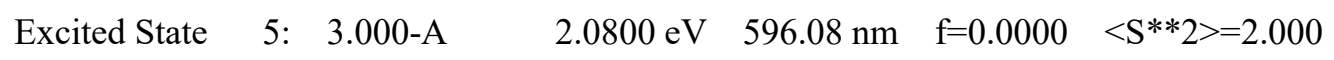

$\begin{array}{lc}120 \mathrm{~A}->129 \mathrm{~A} & -0.20729 \\ 122 \mathrm{~A}->129 \mathrm{~A} & -0.34159 \\ 125 \mathrm{~A}->129 \mathrm{~A} & 0.15497 \\ 126 \mathrm{~A}->129 \mathrm{~A} & 0.36430 \\ 127 \mathrm{~A}-129 \mathrm{~A} & -0.31874 \\ 128 \mathrm{~A}->130 \mathrm{~A} & 0.13707 \\ 120 \mathrm{~B}->129 \mathrm{~B} & 0.20729 \\ 122 \mathrm{~B}->129 \mathrm{~B} & 0.34159 \\ 125 \mathrm{~B}->129 \mathrm{~B} & -0.15497\end{array}$




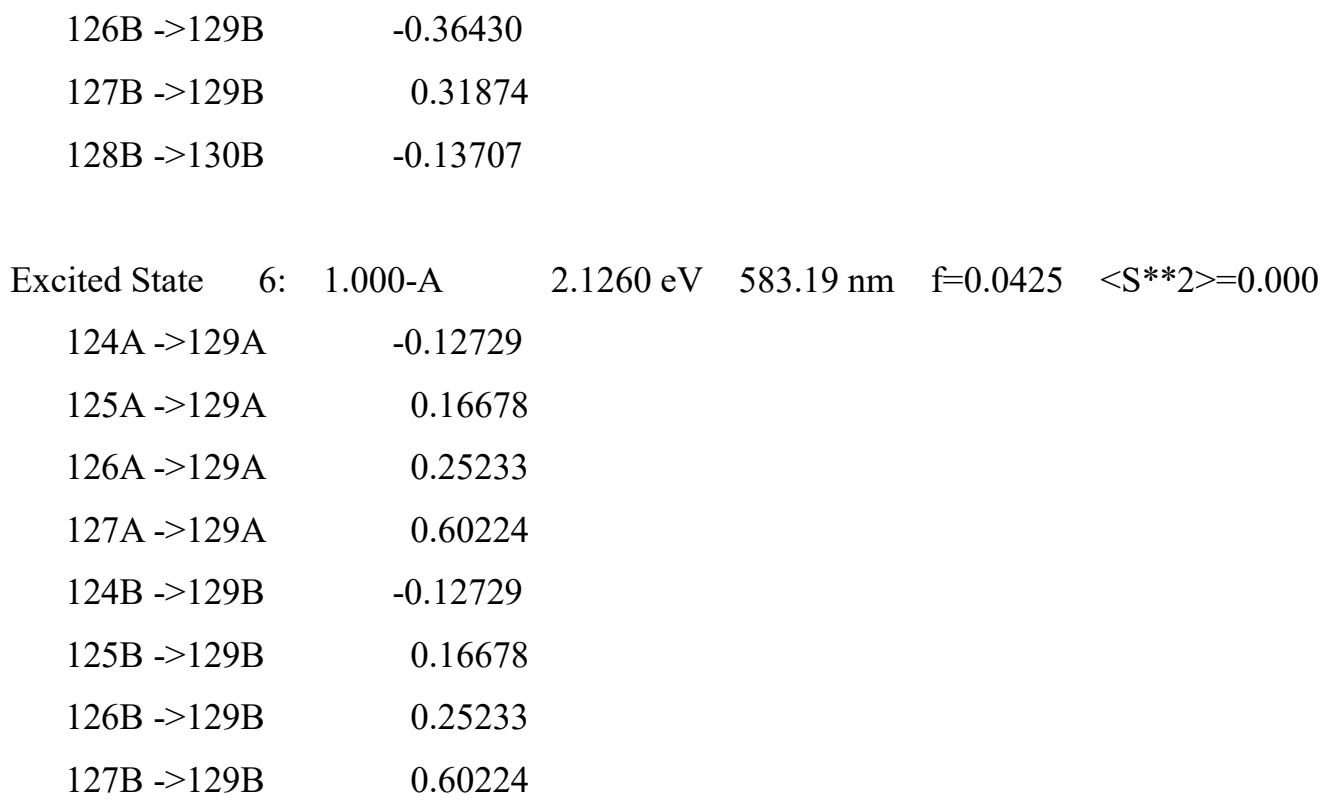

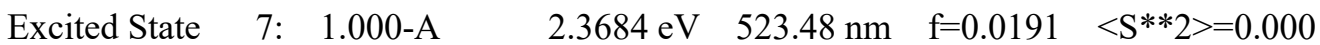

$\begin{array}{lr}120 \mathrm{~A}->129 \mathrm{~A} & -0.12574 \\ 121 \mathrm{~A}->129 \mathrm{~A} & -0.16558 \\ 124 \mathrm{~A}->129 \mathrm{~A} & -0.18160 \\ 125 \mathrm{~A}->129 \mathrm{~A} & 0.46866 \\ 126 \mathrm{~A}->129 \mathrm{~A} & 0.30894 \\ 127 \mathrm{~A}->129 \mathrm{~A} & -0.30698 \\ 120 \mathrm{~B}->129 \mathrm{~B} & -0.12574 \\ 121 \mathrm{~B}->129 \mathrm{~B} & -0.16558 \\ 124 \mathrm{~B}->129 \mathrm{~B} & -0.18160 \\ 125 \mathrm{~B}->129 \mathrm{~B} & 0.46866 \\ 126 \mathrm{~B}->129 \mathrm{~B} & 0.30894 \\ 127 \mathrm{~B}->129 \mathrm{~B} & -0.30698\end{array}$

Excited State $\quad 8: \quad 3.000-\mathrm{A} \quad 2.3797 \mathrm{eV} \quad 521.02 \mathrm{~nm} \quad \mathrm{f}=0.0000 \quad<\mathrm{S}^{* *} 2>=2.000$

$\begin{array}{lc}121 \mathrm{~A}->129 \mathrm{~A} & -0.28166 \\ 125 \mathrm{~A}->129 \mathrm{~A} & 0.50418 \\ 126 \mathrm{~A}->129 \mathrm{~A} & -0.32912 \\ 121 \mathrm{~B}->129 \mathrm{~B} & 0.28166 \\ 125 \mathrm{~B}->129 \mathrm{~B} & -0.50418 \\ 126 \mathrm{~B}->129 \mathrm{~B} & 0.32912\end{array}$

Excited State $\quad 9: \quad 1.000-\mathrm{A} \quad 2.6094 \mathrm{eV} \quad 475.14 \mathrm{~nm} \quad \mathrm{f}=0.0587 \quad<\mathrm{S} * * 2>=0.000$ $\begin{array}{ll}121 \mathrm{~A}->129 \mathrm{~A} & 0.33622 \\ 122 \mathrm{~A}->129 \mathrm{~A} & 0.11076\end{array}$ 


$\begin{array}{lr}125 \mathrm{~A}->129 \mathrm{~A} & -0.29576 \\ 126 \mathrm{~A}->129 \mathrm{~A} & 0.45592 \\ 127 \mathrm{~A}->129 \mathrm{~A} & -0.10823 \\ 128 \mathrm{~A}->130 \mathrm{~A} & -0.17086 \\ 121 \mathrm{~B}->129 \mathrm{~B} & 0.33622 \\ 122 \mathrm{~B}->129 \mathrm{~B} & 0.11076 \\ 125 \mathrm{~B}->129 \mathrm{~B} & -0.29576 \\ 126 \mathrm{~B}->129 \mathrm{~B} & 0.45592 \\ 127 \mathrm{~B}->129 \mathrm{~B} & -0.10823 \\ 128 \mathrm{~B}->130 \mathrm{~B} & -0.17086\end{array}$

$\begin{array}{cc}\text { Excited State } \quad 10: & 3.000-\mathrm{A} \\ 121 \mathrm{~A}->129 \mathrm{~A} & -0.16676 \\ 122 \mathrm{~A}->129 \mathrm{~A} & 0.22435 \\ 128 \mathrm{~A}->130 \mathrm{~A} & 0.50643 \\ 128 \mathrm{~A}->132 \mathrm{~A} & -0.19621 \\ 128 \mathrm{~A}->133 \mathrm{~A} & 0.18563 \\ 121 \mathrm{~B}->129 \mathrm{~B} & 0.16676 \\ 122 \mathrm{~B}->129 \mathrm{~B} & -0.22435 \\ 128 \mathrm{~B}->130 \mathrm{~B} & -0.50643 \\ 128 \mathrm{~B}->132 \mathrm{~B} & 0.19621 \\ 128 \mathrm{~B}->133 \mathrm{~B} & -0.18563\end{array}$

Excited State $\quad 11: \quad 3.000-\mathrm{A} \quad 2.6845 \mathrm{eV} \quad 461.85 \mathrm{~nm} \quad \mathrm{f}=0.0000 \quad<\mathrm{S}^{* *} 2>=2.000$

$\begin{array}{lr}115 \mathrm{~A}->129 \mathrm{~A} & 0.45301 \\ 118 \mathrm{~A}->129 \mathrm{~A} & 0.10845 \\ 121 \mathrm{~A}->129 \mathrm{~A} & -0.34123 \\ 124 \mathrm{~A}->129 \mathrm{~A} & 0.21469 \\ 126 \mathrm{~A}->129 \mathrm{~A} & 0.12227 \\ 128 \mathrm{~A}->130 \mathrm{~A} & -0.17416 \\ 115 \mathrm{~B}->129 \mathrm{~B} & -0.45301 \\ 118 \mathrm{~B}->129 \mathrm{~B} & -0.10845 \\ 121 \mathrm{~B}->129 \mathrm{~B} & 0.34123 \\ 124 \mathrm{~B}->129 \mathrm{~B} & -0.21469 \\ 126 \mathrm{~B}->129 \mathrm{~B} & -0.12227 \\ 128 \mathrm{~B}->130 \mathrm{~B} & 0.17416\end{array}$

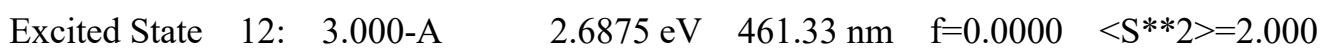
$115 \mathrm{~A}->129 \mathrm{~A} \quad 0.49089$ 


$\begin{array}{lc}121 \mathrm{~A}->129 \mathrm{~A} & 0.27890 \\ 124 \mathrm{~A}->129 \mathrm{~A} & -0.18906 \\ 126 \mathrm{~A}->129 \mathrm{~A} & -0.13012 \\ 128 \mathrm{~A}->130 \mathrm{~A} & 0.26479 \\ 115 \mathrm{~B}->129 \mathrm{~B} & -0.49089 \\ 121 \mathrm{~B}->129 \mathrm{~B} & -0.27890 \\ 124 \mathrm{~B}->129 \mathrm{~B} & 0.18906 \\ 126 \mathrm{~B}->129 \mathrm{~B} & 0.13012 \\ 128 \mathrm{~B}->130 \mathrm{~B} & -0.26479\end{array}$

Excited State $\quad 13: \quad 1.000-\mathrm{A} \quad 2.8348 \mathrm{eV} \quad 437.36 \mathrm{~nm} \quad \mathrm{f}=0.1044 \quad<\mathrm{S} * * 2>=0.000$

$\begin{array}{lc}121 \mathrm{~A}->129 \mathrm{~A} & 0.22484 \\ 122 \mathrm{~A}->129 \mathrm{~A} & 0.48852 \\ 124 \mathrm{~A}->129 \mathrm{~A} & -0.24533 \\ 126 \mathrm{~A}->129 \mathrm{~A} & -0.16319 \\ 128 \mathrm{~A}->130 \mathrm{~A} & 0.28914 \\ 121 \mathrm{~B}->129 \mathrm{~B} & 0.22484 \\ 122 \mathrm{~B}->129 \mathrm{~B} & 0.48852 \\ 124 \mathrm{~B}->129 \mathrm{~B} & -0.24533 \\ 126 \mathrm{~B}->129 \mathrm{~B} & -0.16319 \\ 128 \mathrm{~B}->130 \mathrm{~B} & 0.28914\end{array}$

Excited State $\quad 14: \quad 3.000-\mathrm{A} \quad 2.9346 \mathrm{eV} \quad 422.49 \mathrm{~nm} \quad \mathrm{f}=0.0000 \quad<\mathrm{S}^{* *} 2>=2.000$

$\begin{array}{ll}121 \mathrm{~A}->129 \mathrm{~A} & 0.30286 \\ 123 \mathrm{~A}->129 \mathrm{~A} & 0.33837 \\ 124 \mathrm{~A}->129 \mathrm{~A} & 0.37942 \\ 125 \mathrm{~A}->129 \mathrm{~A} & 0.24593 \\ 128 \mathrm{~A}->132 \mathrm{~A} & -0.13265 \\ 121 \mathrm{~B}->129 \mathrm{~B} & -0.30286 \\ 123 \mathrm{~B}->129 \mathrm{~B} & -0.33837 \\ 124 \mathrm{~B}->129 \mathrm{~B} & -0.37942 \\ 125 \mathrm{~B}->129 \mathrm{~B} & -0.24593 \\ 128 \mathrm{~B}->132 \mathrm{~B} & 0.13265\end{array}$

Excited State $\quad 15: \quad 3.000-\mathrm{A} \quad 2.9829 \mathrm{eV} \quad 415.65 \mathrm{~nm} \quad \mathrm{f}=0.0000 \quad<\mathrm{S} * * 2>=2.000$
$117 \mathrm{~A}->129 \mathrm{~A}$
0.16671
$118 \mathrm{~A}->129 \mathrm{~A}$
$-0.18036$
$120 \mathrm{~A}->129 \mathrm{~A}$
$-0.12792$
$122 \mathrm{~A} \rightarrow 129 \mathrm{~A}$
0.19486 


$\begin{array}{lc}123 \mathrm{~A}->129 \mathrm{~A} & 0.15214 \\ 124 \mathrm{~A}->129 \mathrm{~A} & 0.20978 \\ 128 \mathrm{~A}->131 \mathrm{~A} & -0.21375 \\ 128 \mathrm{~A}->132 \mathrm{~A} & 0.42215 \\ 128 \mathrm{~A}->134 \mathrm{~A} & -0.10611 \\ 117 \mathrm{~B}->129 \mathrm{~B} & -0.16671 \\ 118 \mathrm{~B}->129 \mathrm{~B} & 0.18036 \\ 120 \mathrm{~B}->129 \mathrm{~B} & 0.12792 \\ 122 \mathrm{~B}->129 \mathrm{~B} & -0.19486 \\ 123 \mathrm{~B}->129 \mathrm{~B} & -0.15214 \\ 124 \mathrm{~B}->129 \mathrm{~B} & -0.20978 \\ 128 \mathrm{~B}->131 \mathrm{~B} & 0.21375 \\ 128 \mathrm{~B}->132 \mathrm{~B} & -0.42215 \\ 128 \mathrm{~B}->134 \mathrm{~B} & 0.10611\end{array}$

$\begin{array}{cr}\text { Excited State 16: } & 1.000-\mathrm{A} \\ 121 \mathrm{~A}->129 \mathrm{~A} & -0.17604 \\ 122 \mathrm{~A}->129 \mathrm{~A} & 0.38598 \\ 123 \mathrm{~A}->129 \mathrm{~A} & 0.26580 \\ 124 \mathrm{~A}->129 \mathrm{~A} & 0.46926 \\ 126 \mathrm{~A}->129 \mathrm{~A} & 0.13617 \\ 121 \mathrm{~B}->129 \mathrm{~B} & -0.17604 \\ 122 \mathrm{~B}->129 \mathrm{~B} & 0.38598 \\ 123 \mathrm{~B}->129 \mathrm{~B} & 0.26580 \\ 124 \mathrm{~B}->129 \mathrm{~B} & 0.46926 \\ 126 \mathrm{~B}->129 \mathrm{~B} & 0.13617\end{array}$

Excited State $\quad 17: \quad 3.000-\mathrm{A} \quad 3.0293 \mathrm{eV} \quad 409.29 \mathrm{~nm} \quad \mathrm{f}=0.0000 \quad<\mathrm{S} * * 2>=2.000$

$\begin{array}{lr}116 \mathrm{~A}->129 \mathrm{~A} & 0.20072 \\ 117 \mathrm{~A}->129 \mathrm{~A} & 0.20069 \\ 118 \mathrm{~A}->129 \mathrm{~A} & 0.32213 \\ 119 \mathrm{~A}->129 \mathrm{~A} & -0.11604 \\ 120 \mathrm{~A}->129 \mathrm{~A} & 0.34316 \\ 123 \mathrm{~A}->129 \mathrm{~A} & -0.13193 \\ 125 \mathrm{~A}->129 \mathrm{~A} & 0.10032 \\ 128 \mathrm{~A}->130 \mathrm{~A} & 0.13212 \\ 128 \mathrm{~A}->132 \mathrm{~A} & 0.15260 \\ 128 \mathrm{~A}->133 \mathrm{~A} & 0.15704 \\ 116 \mathrm{~B}->129 \mathrm{~B} & -0.20072\end{array}$




$$
\begin{array}{lr}
117 \mathrm{~B}->129 \mathrm{~B} & -0.20069 \\
118 \mathrm{~B}->129 \mathrm{~B} & -0.32213 \\
119 \mathrm{~B}->129 \mathrm{~B} & 0.11604 \\
120 \mathrm{~B}->129 \mathrm{~B} & -0.34316 \\
123 \mathrm{~B}->129 \mathrm{~B} & 0.13193 \\
125 \mathrm{~B}->129 \mathrm{~B} & -0.10032 \\
128 \mathrm{~B}->130 \mathrm{~B} & -0.13212 \\
128 \mathrm{~B}->132 \mathrm{~B} & -0.15260 \\
128 \mathrm{~B}->133 \mathrm{~B} & -0.15704
\end{array}
$$

Excited State $\quad 18: \quad 1.000-\mathrm{A} \quad 3.0682 \mathrm{eV} \quad 404.10 \mathrm{~nm} \quad \mathrm{f}=0.0096 \quad<\mathrm{S}^{* *} 2>=0.000$

$\begin{array}{lr}121 \mathrm{~A}->129 \mathrm{~A} & 0.46201 \\ 122 \mathrm{~A}->129 \mathrm{~A} & -0.21024 \\ 123 \mathrm{~A}->129 \mathrm{~A} & 0.27382 \\ 124 \mathrm{~A}->129 \mathrm{~A} & 0.19350 \\ 125 \mathrm{~A}->129 \mathrm{~A} & 0.28363 \\ 128 \mathrm{~A}->130 \mathrm{~A} & 0.16943 \\ 121 \mathrm{~B}->129 \mathrm{~B} & 0.46201 \\ 122 \mathrm{~B}->129 \mathrm{~B} & -0.21024 \\ 123 \mathrm{~B}->129 \mathrm{~B} & 0.27382 \\ 124 \mathrm{~B}->129 \mathrm{~B} & 0.19350 \\ 125 \mathrm{~B}->129 \mathrm{~B} & 0.28363 \\ 128 \mathrm{~B}->130 \mathrm{~B} & 0.16943\end{array}$

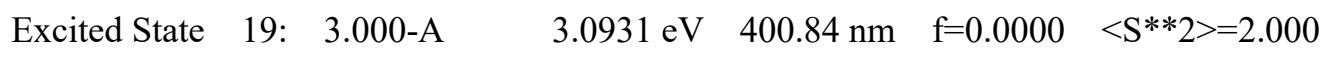
$116 \mathrm{~A}->129 \mathrm{~A} \quad 0.16284$
$117 \mathrm{~A}->129 \mathrm{~A} \quad 0.38176$
$118 \mathrm{~A}->129 \mathrm{~A} \quad 0.13184$
$120 \mathrm{~A} \rightarrow 129 \mathrm{~A} \quad-0.20391$
$128 \mathrm{~A} \rightarrow 133 \mathrm{~A} \quad-0.26122$
$128 \mathrm{~A} \rightarrow 134 \mathrm{~A} \quad 0.21890$
$128 \mathrm{~A}->135 \mathrm{~A} \quad 0.16542$
$116 \mathrm{~B}->129 \mathrm{~B} \quad-0.16284$
$117 \mathrm{~B}->129 \mathrm{~B} \quad-0.38176$
$118 \mathrm{~B}->129 \mathrm{~B} \quad-0.13184$
$120 \mathrm{~B}->129 \mathrm{~B} \quad 0.20391$
$128 \mathrm{~B}->133 \mathrm{~B} \quad 0.26122$
$128 \mathrm{~B}->134 \mathrm{~B} \quad-0.21890$
$128 \mathrm{~B}->135 \mathrm{~B} \quad-0.16542$ 


$\begin{array}{ccccc}\text { Excited State } 20: & 1.000-\mathrm{A} & 3.1321 \mathrm{eV} & 395.85 \mathrm{~nm} & \mathrm{f}=0.2114 \quad<\mathrm{S}^{* *} 2>=0.000 \\ 120 \mathrm{~A}->129 \mathrm{~A} & -0.10831 & & \\ 121 \mathrm{~A}->129 \mathrm{~A} & -0.13304 & & \\ 122 \mathrm{~A}->129 \mathrm{~A} & -0.16567 & & \\ 125 \mathrm{~A}->129 \mathrm{~A} & -0.20264 & \\ 126 \mathrm{~A}->129 \mathrm{~A} & 0.20372 & \\ 128 \mathrm{~A}->130 \mathrm{~A} & 0.57590 \\ 128 \mathrm{~A}->132 \mathrm{~A} & 0.10154 \\ 120 \mathrm{~B}->129 \mathrm{~B} & -0.10831 \\ 121 \mathrm{~B}->129 \mathrm{~B} & -0.13304 \\ 122 \mathrm{~B}->129 \mathrm{~B} & -0.16567 & \\ 125 \mathrm{~B}->129 \mathrm{~B} & -0.20264 & \\ 126 \mathrm{~B}->129 \mathrm{~B} & 0.20372 & \\ 128 \mathrm{~B}->130 \mathrm{~B} & 0.57590 & \\ 128 \mathrm{~B}->132 \mathrm{~B} & 0.10154\end{array}$
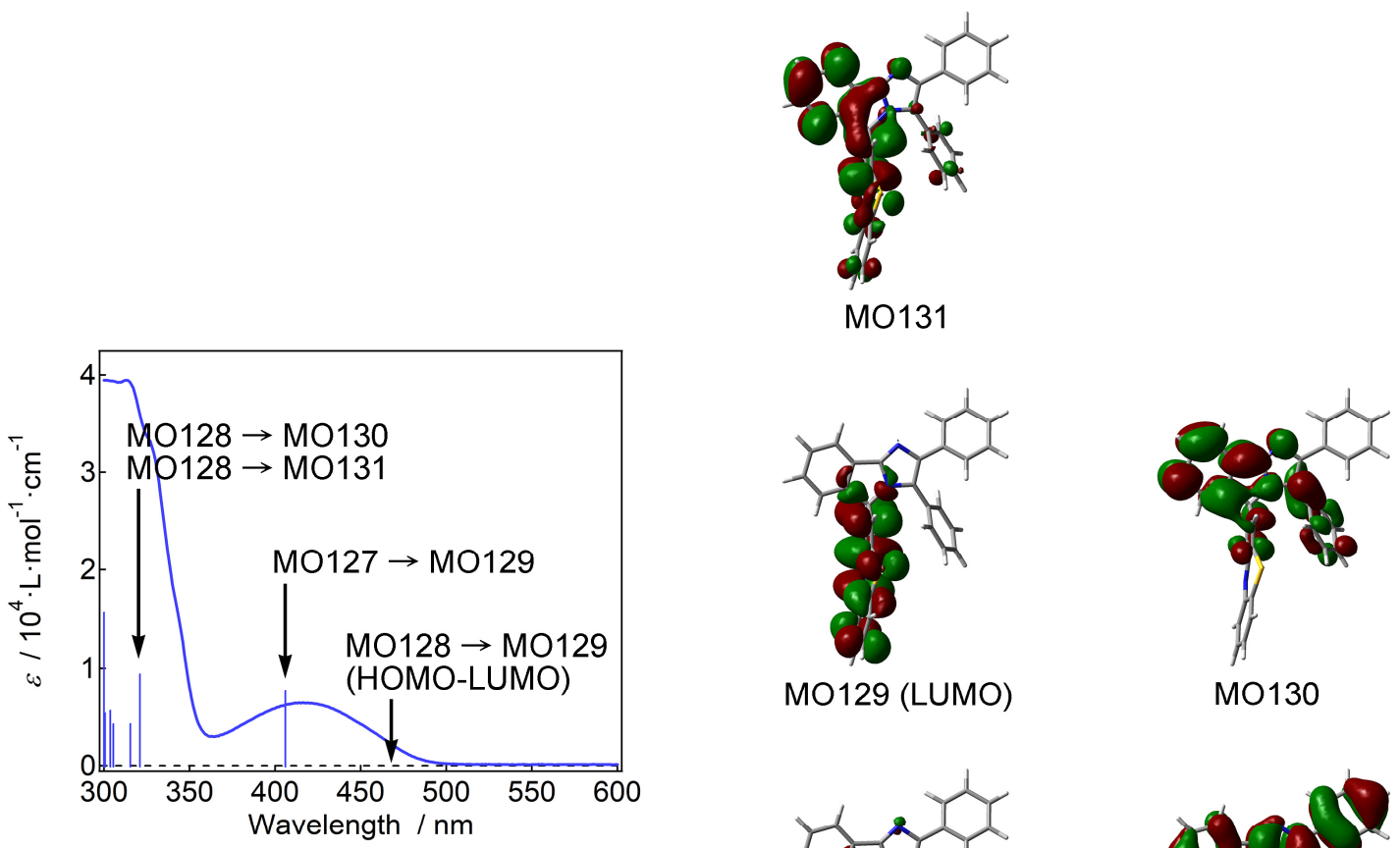

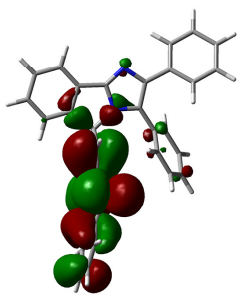

M0127

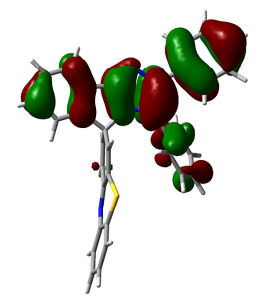

MO128 (HOMO)

Figure S49. (a) UV-vis absorption spectrum of 1 in benzene at room temperature. The calculated spectrum (MPW1PW91/6-31G+(d)//M05-2X/6-31G(d) level of the theory) is shown by the blue vertical lines. (b) The relevant molecular orbitals of the 1 calculated at the M05-2X/6-31G(d) level of the theory. 
Table S6. Standard orientation of the optimized geometry for the triplet biradical form of the ring-opening form of 1.

\begin{tabular}{|c|c|c|c|c|}
\hline \multirow{2}{*}{ Tag } & \multirow{2}{*}{ Symbol } & \multicolumn{3}{|c|}{ Coordinates (Angstroms) } \\
\hline & & $\mathrm{X}$ & $\mathrm{Y}$ & Z \\
\hline 1 & $\mathrm{C}$ & -2.3310400 & 5.2234260 & -0.1515350 \\
\hline 2 & $\mathrm{C}$ & -0.2371270 & 3.3978630 & 0.2962750 \\
\hline 3 & $\mathrm{C}$ & -1.0478940 & 5.6845760 & 0.1221080 \\
\hline 4 & $\mathrm{C}$ & -2.5745070 & 3.8619360 & -0.1810820 \\
\hline 5 & $\mathrm{C}$ & -1.5515750 & 2.9301910 & 0.0617050 \\
\hline 6 & $\mathrm{C}$ & -0.0193050 & 4.7752270 & 0.3360800 \\
\hline 7 & $\mathrm{C}$ & 0.9520670 & 2.5167720 & 0.4489690 \\
\hline 8 & $\mathrm{C}$ & 3.3001960 & 0.9436110 & 0.6945350 \\
\hline 9 & $\mathrm{C}$ & 1.3588980 & 1.6874850 & -0.5900950 \\
\hline 10 & $\mathrm{C}$ & 1.7354760 & 2.5731040 & 1.6134010 \\
\hline 11 & $\mathrm{C}$ & 2.8694190 & 1.8049690 & 1.7322460 \\
\hline 12 & $\mathrm{C}$ & 2.5063240 & 0.9090450 & -0.4823560 \\
\hline 13 & S & 2.9288480 & -0.1070210 & -1.8489380 \\
\hline 14 & $\mathrm{C}$ & 4.3988640 & -0.8805800 & -1.2875010 \\
\hline 15 & $\mathrm{C}$ & 4.9424300 & -0.6129950 & -0.0048520 \\
\hline 16 & $\mathrm{~N}$ & 4.4337460 & 0.2363960 & 0.9235830 \\
\hline 17 & $\mathrm{C}$ & 6.1332020 & -1.2938110 & 0.3511330 \\
\hline 18 & $\mathrm{C}$ & 6.7446400 & -2.1797770 & -0.5055130 \\
\hline 19 & $\mathrm{C}$ & 6.1888390 & -2.4274100 & -1.7672590 \\
\hline 20 & $\mathrm{C}$ & 5.0259720 & -1.7808090 & -2.1497850 \\
\hline 21 & $\mathrm{C}$ & -1.9361700 & 1.5272640 & 0.1152730 \\
\hline 22 & $\mathrm{~N}$ & -3.1463180 & 1.1079220 & -0.3644520 \\
\hline 23 & $\mathrm{C}$ & -3.1655790 & -0.1920170 & -0.1320160 \\
\hline 24 & $\mathrm{C}$ & -1.9128590 & -0.5481010 & 0.5722740 \\
\hline 25 & $\mathrm{~N}$ & -1.1800150 & 0.5489290 & 0.6777570 \\
\hline 26 & $\mathrm{C}$ & -4.2702260 & -1.0258730 & -0.6061030 \\
\hline 27 & $\mathrm{C}$ & -6.4114000 & -2.5243930 & -1.6037470 \\
\hline 28 & $\mathrm{C}$ & -4.0818740 & -2.3525440 & -1.0130510 \\
\hline 29 & $\mathrm{C}$ & -5.5451570 & -0.4560270 & -0.7264010 \\
\hline 30 & $\mathrm{C}$ & -6.6068210 & -1.2022130 & -1.2122690 \\
\hline 31 & $\mathrm{C}$ & -5.1461230 & -3.0933530 & -1.5095300 \\
\hline 32 & $\mathrm{C}$ & -1.4622780 & -1.8064140 & 1.1622400 \\
\hline 33 & $\mathrm{C}$ & -0.5091660 & -4.1429030 & 2.3686860 \\
\hline 34 & $\mathrm{C}$ & -0.0841540 & -2.0196190 & 1.3153070 \\
\hline
\end{tabular}




\begin{tabular}{|c|c|c|c|c|}
\hline 35 & $\mathrm{C}$ & -2.3542360 & -2.7734370 & 1.6441330 \\
\hline 36 & $\mathrm{C}$ & -1.8780440 & -3.9314520 & 2.2436190 \\
\hline 37 & $\mathrm{C}$ & 0.3859350 & -3.1817530 & 1.9048790 \\
\hline 38 & $\mathrm{H}$ & -3.1399750 & 5.9235090 & -0.3339900 \\
\hline 39 & $\mathrm{H}$ & -0.8417260 & 6.7499020 & 0.1547000 \\
\hline 40 & $\mathrm{H}$ & -3.5688090 & 3.4787140 & -0.3762920 \\
\hline 41 & $\mathrm{H}$ & 0.9896750 & 5.1346630 & 0.5125420 \\
\hline 42 & $\mathrm{H}$ & 0.7678050 & 1.6446860 & -1.5001190 \\
\hline 43 & $\mathrm{H}$ & 1.4265570 & 3.2175440 & 2.4300960 \\
\hline 44 & $\mathrm{H}$ & 3.4792120 & 1.8276620 & 2.6285330 \\
\hline 45 & $\mathrm{H}$ & 6.5368110 & -1.0783620 & 1.3342630 \\
\hline 46 & $\mathrm{H}$ & 7.6564020 & -2.6856980 & -0.2047070 \\
\hline 47 & $\mathrm{H}$ & 6.6656530 & -3.1244510 & -2.4485580 \\
\hline 48 & $\mathrm{H}$ & 4.5936680 & -1.9714310 & -3.1279180 \\
\hline 49 & $\mathrm{H}$ & -7.2430220 & -3.1072030 & -1.9876010 \\
\hline 50 & $\mathrm{H}$ & -3.0950040 & -2.7976530 & -0.9602820 \\
\hline 51 & $\mathrm{H}$ & -5.6797330 & 0.5785050 & -0.4320800 \\
\hline 52 & $\mathrm{H}$ & -7.5914040 & -0.7517360 & -1.2902060 \\
\hline 53 & $\mathrm{H}$ & -4.9838060 & -4.1176640 & -1.8299660 \\
\hline 54 & $\mathrm{H}$ & -0.1394640 & -5.0515400 & 2.8338900 \\
\hline 55 & $\mathrm{H}$ & 0.6010160 & -1.2557800 & 0.9651860 \\
\hline 56 & $\mathrm{H}$ & -3.4226070 & -2.6076380 & 1.5700350 \\
\hline 57 & $\mathrm{H}$ & -2.5801900 & -4.6682200 & 2.6210920 \\
\hline 58 & $\mathrm{H}$ & 1.4551120 & -3.3381420 & 2.0075660 \\
\hline
\end{tabular}

SCF Done: E(UCAM-B3LYP)

$=-1831.76538901 \quad$ A.U.

$\begin{array}{lll}\text { Zero-point correction } & =0.452539 \text { (Hartree/Particle) } \\ \text { Thermal correction to Energy } & =0.480367 \\ \text { Thermal correction to Enthalpy } & =0.481311 \\ \text { Thermal correction to Gibbs Free Energy } & =0.388981 \\ \text { Sum of electronic and zero-point Energies } & =-1831.313154 \\ \text { Sum of electronic and thermal Energies } & =-1831.285326 \\ \text { Sum of electronic and thermal Enthalpies } & =-1831.284382 \\ \text { Sum of electronic and thermal Free Energies } & =-1831.376712\end{array}$

$\begin{array}{lllllll}\text { Low frequencies --- } & -1.1130 & -0.0013 & -0.0008 & 0.0017 & 0.3375 & 0.4471\end{array}$

Low frequencies --- $\quad 8.6238 \quad 14.4921 \quad 17.8116$ 
The Results of the TDDFT calculation

Excited State $\quad 1: \quad 3.122-\mathrm{A} \quad 2.0764 \mathrm{eV} \quad 597.10 \mathrm{~nm} \quad \mathrm{f}=0.0126 \quad<\mathrm{S} * * 2>=2.187$

$\begin{array}{lr}128 \mathrm{~A}->130 \mathrm{~A} & 0.12958 \\ 115 \mathrm{~B}->128 \mathrm{~B} & -0.20664 \\ 116 \mathrm{~B}->128 \mathrm{~B} & 0.27628 \\ 125 \mathrm{~B}->128 \mathrm{~B} & 0.72844 \\ 126 \mathrm{~B}->128 \mathrm{~B} & -0.36354 \\ 127 \mathrm{~B}->128 \mathrm{~B} & 0.32026\end{array}$

This state for optimization and/or second-order correction.

Total Energy, E(TD-HF/TD-DFT) $=-1831.73633980$

Copying the excited state density for this state as the 1-particle RhoCI density.

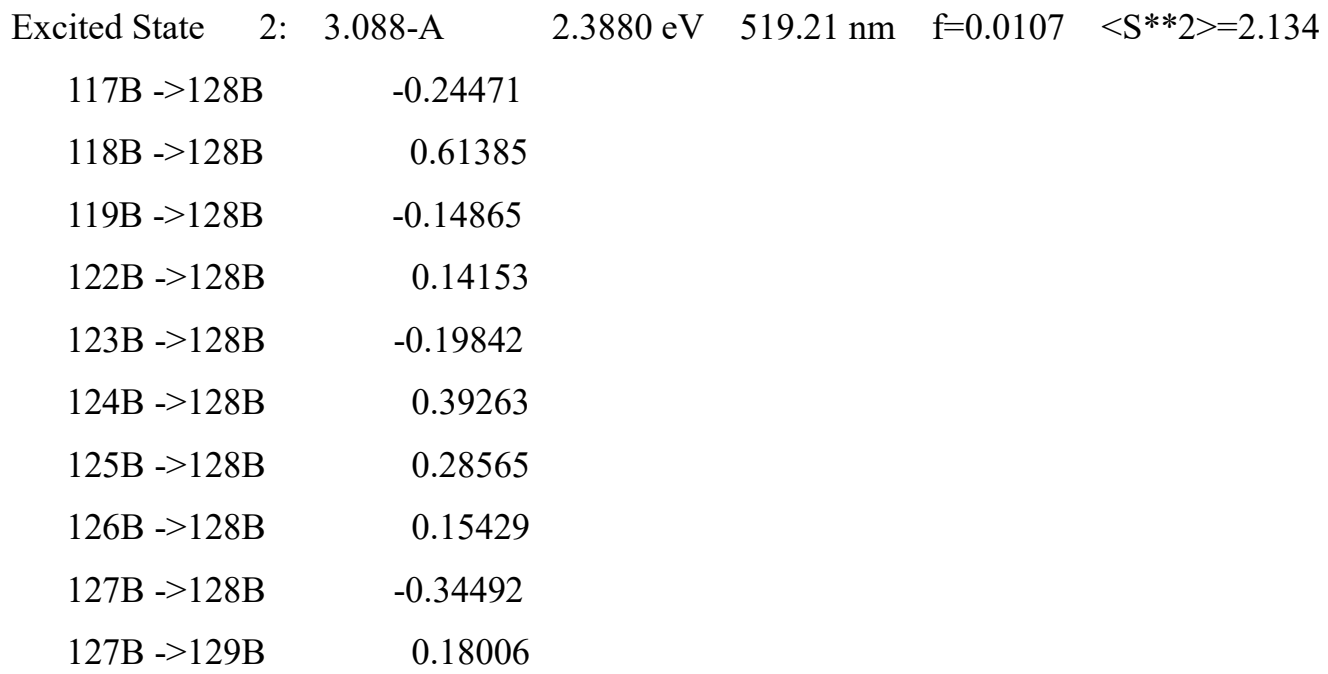

Excited State $\quad 3: \quad 3.140-\mathrm{A} \quad 2.5195 \mathrm{eV} \quad 492.11 \mathrm{~nm} \quad \mathrm{f}=0.0230 \quad<\mathrm{S}^{* *} 2>=2.215$

$\begin{array}{lc}118 \mathrm{~B}->128 \mathrm{~B} & -0.18246 \\ 124 \mathrm{~B}->128 \mathrm{~B} & -0.32417 \\ 125 \mathrm{~B}->128 \mathrm{~B} & 0.10978 \\ 127 \mathrm{~B}->128 \mathrm{~B} & -0.29021 \\ 127 \mathrm{~B}->129 \mathrm{~B} & 0.79276\end{array}$

Excited State $\quad 4: \quad 3.230-\mathrm{A} \quad 2.6864 \mathrm{eV} \quad 461.53 \mathrm{~nm} \quad \mathrm{f}=0.1089 \quad<\mathrm{S} * * 2>=2.358$

$\begin{array}{lr}126 \mathrm{~A}->130 \mathrm{~A} & -0.10088 \\ 128 \mathrm{~A}->131 \mathrm{~A} & 0.18510 \\ 129 \mathrm{~A}->131 \mathrm{~A} & 0.13209 \\ 116 \mathrm{~B}->128 \mathrm{~B} & -0.11064 \\ 118 \mathrm{~B}->128 \mathrm{~B} & -0.16955 \\ 124 \mathrm{~B}->128 \mathrm{~B} & 0.55562\end{array}$




$$
\begin{array}{lr}
125 \mathrm{~B}->128 \mathrm{~B} & -0.20535 \\
126 \mathrm{~B}->128 \mathrm{~B} & -0.37707 \\
126 \mathrm{~B}->129 \mathrm{~B} & 0.23028 \\
127 \mathrm{~B}->128 \mathrm{~B} & 0.18432 \\
127 \mathrm{~B}->129 \mathrm{~B} & 0.38467
\end{array}
$$

Excited State $\quad 5: \quad 3.087-\mathrm{A} \quad 2.7074 \mathrm{eV} \quad 457.94 \mathrm{~nm} \quad \mathrm{f}=0.0036 \quad<\mathrm{S} * * 2>=2.133$

$$
\begin{array}{lc}
120 \mathrm{~B}->128 \mathrm{~B} & -0.17573 \\
120 \mathrm{~B}->129 \mathrm{~B} & 0.91537 \\
120 \mathrm{~B}->162 \mathrm{~B} & -0.12321 \\
122 \mathrm{~B}->129 \mathrm{~B} & 0.18817
\end{array}
$$

$\begin{array}{cr}\text { Excited State } \quad 6: & 3.236-\mathrm{A} \\ 129 \mathrm{~A}->131 \mathrm{~A} & 0.37884 \\ 129 \mathrm{~A}->132 \mathrm{~A} & 0.49659 \\ 129 \mathrm{~A}->133 \mathrm{~A} & 0.18894 \\ 129 \mathrm{~A}->134 \mathrm{~A} & 0.10138 \\ 122 \mathrm{~B}->129 \mathrm{~B} & 0.16603 \\ 124 \mathrm{~B}->128 \mathrm{~B} & -0.24353 \\ 125 \mathrm{~B}->129 \mathrm{~B} & 0.14832 \\ 126 \mathrm{~B}->129 \mathrm{~B} & 0.45960 \\ 127 \mathrm{~B}->128 \mathrm{~B} & -0.20740\end{array}$

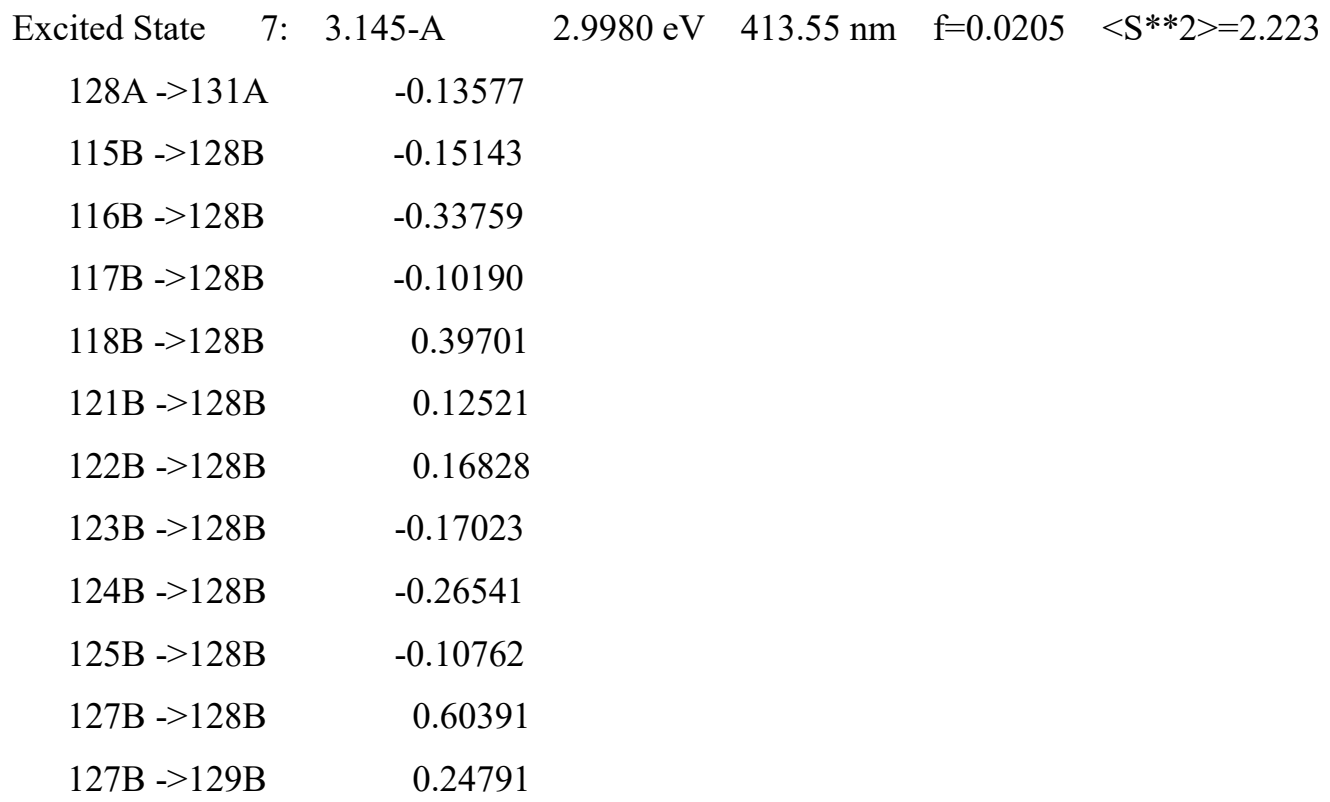

Excited State $\quad 8: \quad 3.192-\mathrm{A} \quad 3.1312 \mathrm{eV} \quad 395.96 \mathrm{~nm} \quad \mathrm{f}=0.0126 \quad<\mathrm{S} * * 2>=2.297$ $128 \mathrm{~A} \rightarrow 130 \mathrm{~A} \quad-0.10631$ 


$$
\begin{array}{lc}
114 \mathrm{~B}->128 \mathrm{~B} & -0.17813 \\
115 \mathrm{~B}->128 \mathrm{~B} & 0.14280 \\
116 \mathrm{~B}->128 \mathrm{~B} & 0.67323 \\
121 \mathrm{~B}->128 \mathrm{~B} & -0.12374 \\
122 \mathrm{~B}->128 \mathrm{~B} & 0.47105 \\
123 \mathrm{~B}->128 \mathrm{~B} & -0.11914 \\
125 \mathrm{~B}->128 \mathrm{~B} & -0.19531 \\
127 \mathrm{~B}->128 \mathrm{~B} & 0.16959
\end{array}
$$

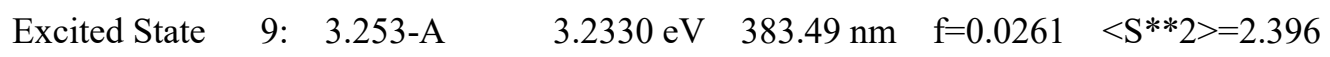

$$
\begin{array}{lr}
126 \mathrm{~A}->130 \mathrm{~A} & -0.10008 \\
129 \mathrm{~A}->131 \mathrm{~A} & 0.11800 \\
129 \mathrm{~A}->132 \mathrm{~A} & 0.21958 \\
129 \mathrm{~A}->133 \mathrm{~A} & 0.11973 \\
116 \mathrm{~B}->128 \mathrm{~B} & 0.25828 \\
117 \mathrm{~B}->128 \mathrm{~B} & 0.16180 \\
119 \mathrm{~B}->128 \mathrm{~B} & 0.18968 \\
122 \mathrm{~B}->128 \mathrm{~B} & -0.34304 \\
122 \mathrm{~B}->131 \mathrm{~B} & 0.10718 \\
123 \mathrm{~B}->128 \mathrm{~B} & 0.10668 \\
124 \mathrm{~B}->128 \mathrm{~B} & 0.19725 \\
125 \mathrm{~B}->130 \mathrm{~B} & -0.10579 \\
126 \mathrm{~B}->128 \mathrm{~B} & 0.52544 \\
126 \mathrm{~B}->131 \mathrm{~B} & -0.10733 \\
127 \mathrm{~B}->128 \mathrm{~B} & 0.34211 \\
127 \mathrm{~B}->129 \mathrm{~B} & 0.11476
\end{array}
$$

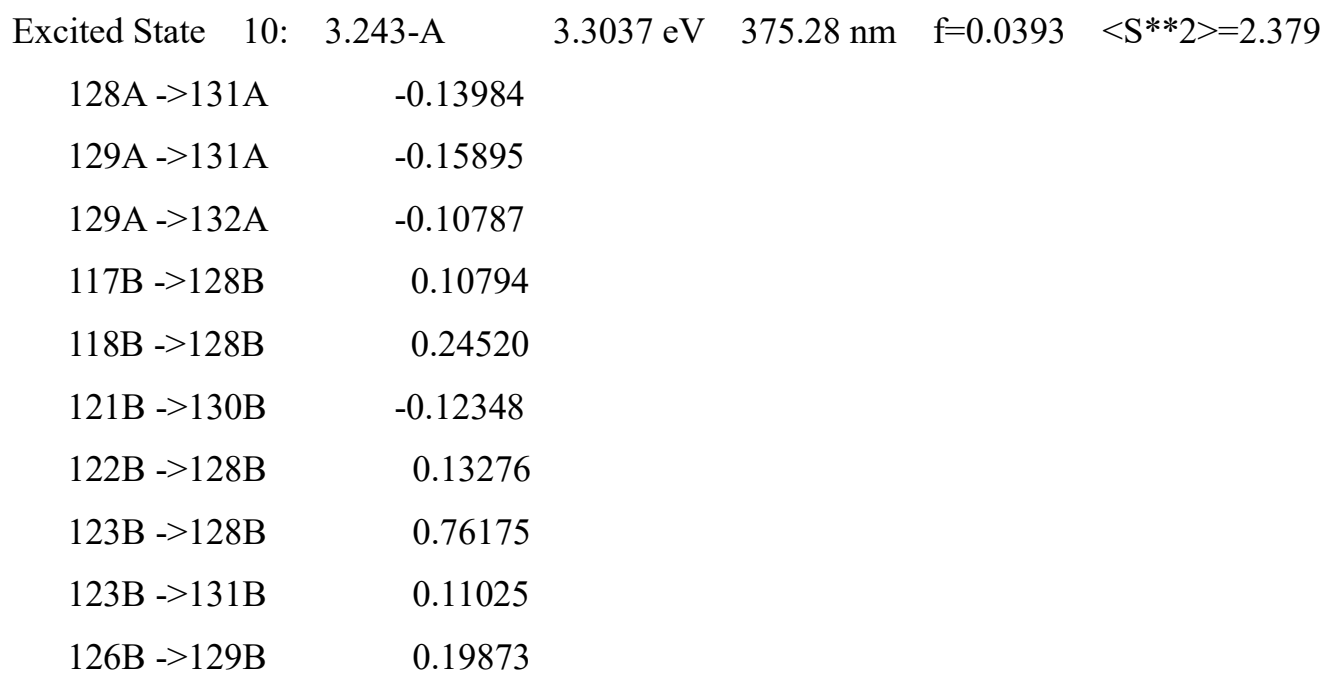




\begin{tabular}{rr} 
Excited State 11: & \multicolumn{1}{l}{$3.412-\mathrm{A}$} \\
$124 \mathrm{~A}->131 \mathrm{~A}$ & -0.15691 \\
$126 \mathrm{~A}->130 \mathrm{~A}$ & 0.13609 \\
$128 \mathrm{~A}->131 \mathrm{~A}$ & 0.16522 \\
$129 \mathrm{~A}->131 \mathrm{~A}$ & 0.25398 \\
$129 \mathrm{~A}->132 \mathrm{~A}$ & 0.19662 \\
$129 \mathrm{~A}->135 \mathrm{~A}$ & 0.12994 \\
$117 \mathrm{~B}->128 \mathrm{~B}$ & -0.33138 \\
$119 \mathrm{~B}->128 \mathrm{~B}$ & -0.11391 \\
$119 \mathrm{~B}->129 \mathrm{~B}$ & 0.14065 \\
$122 \mathrm{~B}->129 \mathrm{~B}$ & -0.12197 \\
$123 \mathrm{~B}->128 \mathrm{~B}$ & 0.43337 \\
$124 \mathrm{~B}->131 \mathrm{~B}$ & -0.12003 \\
$124 \mathrm{~B}->132 \mathrm{~B}$ & 0.10028 \\
$125 \mathrm{~B}->130 \mathrm{~B}$ & 0.15607 \\
$126 \mathrm{~B}->128 \mathrm{~B}$ & 0.10447 \\
$126 \mathrm{~B}->129 \mathrm{~B}$ & -0.33303
\end{tabular}

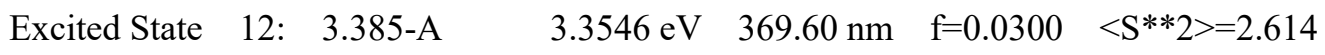
$124 \mathrm{~A}->131 \mathrm{~A} \quad-0.12873$

$126 \mathrm{~A} \rightarrow 130 \mathrm{~A} \quad 0.15003$

$129 \mathrm{~A}->131 \mathrm{~A} \quad-0.12594$

$129 \mathrm{~A}->132 \mathrm{~A} \quad-0.24731$

$129 \mathrm{~A}->133 \mathrm{~A} \quad-0.15007$

$129 \mathrm{~A}->134 \mathrm{~A} \quad-0.12915$

$129 \mathrm{~A}->135 \mathrm{~A} \quad-0.24916$

$117 \mathrm{~B}->128 \mathrm{~B} \quad-0.29581$

$119 \mathrm{~B}->128 \mathrm{~B} \quad 0.13494$

$119 \mathrm{~B}->129 \mathrm{~B} \quad-0.24177$

$122 \mathrm{~B}->128 \mathrm{~B} \quad-0.28029$

$124 \mathrm{~B}->131 \mathrm{~B} \quad-0.13665$

$125 \mathrm{~B}->130 \mathrm{~B} \quad 0.10701$

$126 \mathrm{~B}->128 \mathrm{~B} \quad 0.24460$

$126 \mathrm{~B}->129 \mathrm{~B} \quad 0.38748$

$127 \mathrm{~B}->128 \mathrm{~B} \quad 0.14113$

Excited State $\quad 13: \quad 3.605-\mathrm{A} \quad 3.4318 \mathrm{eV} \quad 361.28 \mathrm{~nm} \quad \mathrm{f}=0.0251 \quad<\mathrm{S} * * 2>=2.999$
$122 \mathrm{~A}->139 \mathrm{~A}$
0.14057
$123 \mathrm{~A}->133 \mathrm{~A}$
$-0.11706$ 


$\begin{array}{lc}123 \mathrm{~A}->134 \mathrm{~A} & 0.11225 \\ 124 \mathrm{~A}->130 \mathrm{~A} & 0.19555 \\ 126 \mathrm{~A}->131 \mathrm{~A} & -0.10569 \\ 126 \mathrm{~A}->140 \mathrm{~A} & 0.11158 \\ 128 \mathrm{~A}->130 \mathrm{~A} & 0.47704 \\ 129 \mathrm{~A}->130 \mathrm{~A} & 0.12780 \\ 114 \mathrm{~B}->128 \mathrm{~B} & -0.16480 \\ 115 \mathrm{~B}->128 \mathrm{~B} & 0.33583 \\ 117 \mathrm{~B}->130 \mathrm{~B} & -0.10738 \\ 121 \mathrm{~B}->128 \mathrm{~B} & -0.28524 \\ 121 \mathrm{~B}->139 \mathrm{~B} & -0.14043 \\ 122 \mathrm{~B}->128 \mathrm{~B} & 0.13478 \\ 123 \mathrm{~B}->133 \mathrm{~B} & -0.16356 \\ 124 \mathrm{~B}->130 \mathrm{~B} & -0.17085 \\ 125 \mathrm{~B}->145 \mathrm{~B} & -0.12118 \\ 126 \mathrm{~B}->128 \mathrm{~B} & 0.14152 \\ 127 \mathrm{~B}->128 \mathrm{~B} & 0.13116\end{array}$

Excited State $\quad 14: \quad 3.252-\mathrm{A} \quad 3.4909 \mathrm{eV} \quad 355.16 \mathrm{~nm} \quad \mathrm{f}=0.0112 \quad<\mathrm{S}^{* *} 2>=2.394$

$\begin{array}{lc}123 \mathrm{~A}->130 \mathrm{~A} & -0.12500 \\ 128 \mathrm{~A}->130 \mathrm{~A} & 0.20674 \\ 116 \mathrm{~B}->128 \mathrm{~B} & 0.18074 \\ 119 \mathrm{~B}->128 \mathrm{~B} & -0.12567 \\ 121 \mathrm{~B}->128 \mathrm{~B} & 0.81394 \\ 121 \mathrm{~B}->130 \mathrm{~B} & 0.12076 \\ 123 \mathrm{~B}->128 \mathrm{~B} & -0.11501 \\ 123 \mathrm{~B}->130 \mathrm{~B} & -0.18005 \\ 125 \mathrm{~B}->128 \mathrm{~B} & -0.14242\end{array}$

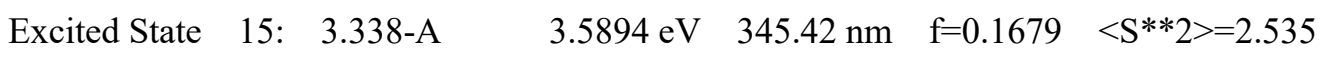

$\begin{array}{lr}126 \mathrm{~A}->130 \mathrm{~A} & 0.12542 \\ 128 \mathrm{~A}->130 \mathrm{~A} & 0.10110 \\ 128 \mathrm{~A}->131 \mathrm{~A} & -0.21279 \\ 129 \mathrm{~A}->131 \mathrm{~A} & -0.28055 \\ 129 \mathrm{~A}->133 \mathrm{~A} & 0.18917 \\ 129 \mathrm{~A}->134 \mathrm{~A} & 0.19031 \\ 129 \mathrm{~A}->135 \mathrm{~A} & 0.20063 \\ 129 \mathrm{~A}->140 \mathrm{~A} & -0.12068 \\ 129 \mathrm{~A}->141 \mathrm{~A} & 0.10883\end{array}$




$\begin{array}{lr}116 \mathrm{~B}->128 \mathrm{~B} & 0.10045 \\ 117 \mathrm{~B}->128 \mathrm{~B} & -0.14561 \\ 119 \mathrm{~B}->128 \mathrm{~B} & -0.10983 \\ 119 \mathrm{~B}->129 \mathrm{~B} & 0.41204 \\ 121 \mathrm{~B}->129 \mathrm{~B} & 0.12861 \\ 122 \mathrm{~B}->128 \mathrm{~B} & -0.13375 \\ 122 \mathrm{~B}->129 \mathrm{~B} & 0.15443 \\ 124 \mathrm{~B}->128 \mathrm{~B} & 0.17113 \\ 125 \mathrm{~B}->128 \mathrm{~B} & -0.10580 \\ 125 \mathrm{~B}->129 \mathrm{~B} & 0.13453 \\ 126 \mathrm{~B}->129 \mathrm{~B} & 0.19439 \\ 127 \mathrm{~B}->131 \mathrm{~B} & 0.12738 \\ 127 \mathrm{~B}->132 \mathrm{~B} & 0.12472\end{array}$

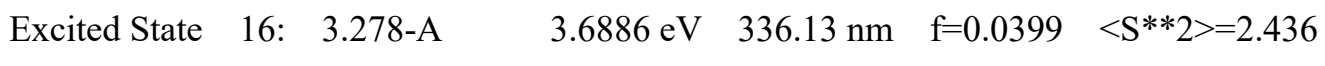

$\begin{array}{ll}125 \mathrm{~A}->135 \mathrm{~A} & -0.11081 \\ 129 \mathrm{~A}->130 \mathrm{~A} & -0.16069 \\ 129 \mathrm{~A}->132 \mathrm{~A} & -0.10970 \\ 129 \mathrm{~A}->133 \mathrm{~A} & 0.53429 \\ 129 \mathrm{~A}->134 \mathrm{~A} & 0.42719 \\ 129 \mathrm{~A}->135 \mathrm{~A} & -0.42373 \\ 119 \mathrm{~B}->129 \mathrm{~B} & -0.23602 \\ 126 \mathrm{~B}->129 \mathrm{~B} & -0.16396 \\ 127 \mathrm{~B}->129 \mathrm{~B} & -0.10791\end{array}$

Excited State $\quad 17: \quad 3.633-\mathrm{A} \quad 3.7411 \mathrm{eV} \quad 331.41 \mathrm{~nm} \quad \mathrm{f}=0.1324 \quad<\mathrm{S} * * 2>=3.050$

$\begin{array}{lr}120 \mathrm{~A}->135 \mathrm{~A} & -0.16505 \\ 125 \mathrm{~A}->131 \mathrm{~A} & -0.10423 \\ 125 \mathrm{~A}->132 \mathrm{~A} & -0.11031 \\ 125 \mathrm{~A}->135 \mathrm{~A} & 0.10913 \\ 126 \mathrm{~A}->135 \mathrm{~A} & -0.10351 \\ 127 \mathrm{~A}->132 \mathrm{~A} & 0.13495 \\ 127 \mathrm{~A}->135 \mathrm{~A} & 0.16445 \\ 128 \mathrm{~A}->131 \mathrm{~A} & 0.22550 \\ 129 \mathrm{~A}->132 \mathrm{~A} & -0.16802 \\ 129 \mathrm{~A}->135 \mathrm{~A} & -0.11658 \\ 129 \mathrm{~A}->141 \mathrm{~A} & 0.10065 \\ 129 \mathrm{~A}->154 \mathrm{~A} & -0.10197 \\ 129 \mathrm{~A}->155 \mathrm{~A} & -0.10136\end{array}$




$\begin{array}{lr}113 \mathrm{~B}->129 \mathrm{~B} & 0.10494 \\ 118 \mathrm{~B}->128 \mathrm{~B} & 0.12332 \\ 119 \mathrm{~B}->129 \mathrm{~B} & 0.38305 \\ 119 \mathrm{~B}->136 \mathrm{~B} & -0.10653 \\ 124 \mathrm{~B}->128 \mathrm{~B} & -0.20801 \\ 125 \mathrm{~B}->129 \mathrm{~B} & 0.11129 \\ 126 \mathrm{~B}->129 \mathrm{~B} & 0.22370 \\ 126 \mathrm{~B}->131 \mathrm{~B} & 0.10541 \\ 126 \mathrm{~B}->132 \mathrm{~B} & 0.13108 \\ 127 \mathrm{~B}->135 \mathrm{~B} & -0.11212 \\ 127 \mathrm{~B}->136 \mathrm{~B} & 0.18881\end{array}$

Excited State $\quad 18: \quad 3.618-\mathrm{A} \quad 3.7900 \mathrm{eV} \quad 327.13 \mathrm{~nm} \quad \mathrm{f}=0.1706 \quad<\mathrm{S} * * 2>=3.022$ $121 \mathrm{~A}->135 \mathrm{~A} \quad-0.10857$

$127 \mathrm{~A} \rightarrow 133 \mathrm{~A} \quad 0.13924$

$127 \mathrm{~A} \rightarrow 134 \mathrm{~A} \quad 0.11188$

$128 \mathrm{~A} \rightarrow 130 \mathrm{~A} \quad-0.16357$

$128 \mathrm{~A}->131 \mathrm{~A} \quad 0.25271$

$128 \mathrm{~A}->132 \mathrm{~A} \quad-0.13944$

$129 \mathrm{~A}->132 \mathrm{~A} \quad-0.13506$

$129 \mathrm{~A}->133 \mathrm{~A} \quad 0.12583$

$129 \mathrm{~A}->134 \mathrm{~A} \quad 0.11565$

$129 \mathrm{~A}->154 \mathrm{~A} \quad 0.12530$

$129 \mathrm{~A} \rightarrow 155 \mathrm{~A} \quad 0.12890$

$113 \mathrm{~B}->129 \mathrm{~B} \quad-0.14495$

$115 \mathrm{~B}->128 \mathrm{~B} \quad 0.11671$

$116 \mathrm{~B}->128 \mathrm{~B} \quad-0.13021$

$119 \mathrm{~B}->129 \mathrm{~B} \quad 0.10929$

119B $->134 \mathrm{~B} \quad-0.10049$

$122 \mathrm{~B}->128 \mathrm{~B} \quad 0.20673$

$122 \mathrm{~B}->131 \mathrm{~B} \quad-0.12571$

$124 \mathrm{~B}->128 \mathrm{~B} \quad-0.20126$

$125 \mathrm{~B}->128 \mathrm{~B} \quad 0.15199$

$126 \mathrm{~B}->128 \mathrm{~B} \quad 0.18612$

126B $->129 \mathrm{~B} \quad 0.11863$

127B $->129 \mathrm{~B} \quad-0.12186$

$127 \mathrm{~B}->136 \mathrm{~B} \quad-0.14338$

Excited State $\quad 19: \quad 3.180-\mathrm{A} \quad 3.9003 \mathrm{eV} \quad 317.88 \mathrm{~nm} \quad \mathrm{f}=0.1125 \quad<\mathrm{S} * * 2>=2.279$ 


$\begin{array}{lc}128 \mathrm{~A}->130 \mathrm{~A} & -0.39663 \\ 128 \mathrm{~A}->131 \mathrm{~A} & -0.16036 \\ 129 \mathrm{~A}->130 \mathrm{~A} & -0.13203 \\ 100 \mathrm{~B}->128 \mathrm{~B} & 0.10523 \\ 114 \mathrm{~B}->128 \mathrm{~B} & -0.15535 \\ 115 \mathrm{~B}->128 \mathrm{~B} & 0.48265 \\ 116 \mathrm{~B}->128 \mathrm{~B} & -0.21271 \\ 117 \mathrm{~B}->128 \mathrm{~B} & 0.15619 \\ 118 \mathrm{~B}->128 \mathrm{~B} & -0.11383 \\ 121 \mathrm{~B}->128 \mathrm{~B} & 0.16157 \\ 122 \mathrm{~B}->128 \mathrm{~B} & 0.18966 \\ 124 \mathrm{~B}->128 \mathrm{~B} & 0.13750 \\ 125 \mathrm{~B}->128 \mathrm{~B} & 0.35153 \\ 126 \mathrm{~B}->128 \mathrm{~B} & 0.12012 \\ 126 \mathrm{~B}->129 \mathrm{~B} & 0.10212\end{array}$

Excited State $\quad 20: \quad 3.452-\mathrm{A}$

$3.9470 \mathrm{eV} \quad 314.12 \mathrm{~nm} \quad \mathrm{f}=0.1377 \quad<\mathrm{S} * * 2>=2.729$

$\begin{array}{lr}120 \mathrm{~A}->133 \mathrm{~A} & -0.12981 \\ 120 \mathrm{~A}->134 \mathrm{~A} & -0.11442 \\ 127 \mathrm{~A}->135 \mathrm{~A} & -0.13893 \\ 128 \mathrm{~A}->131 \mathrm{~A} & 0.12433 \\ 129 \mathrm{~A}->130 \mathrm{~A} & -0.10940 \\ 129 \mathrm{~A}->131 \mathrm{~A} & -0.21858 \\ 129 \mathrm{~A}->134 \mathrm{~A} & 0.10078 \\ 129 \mathrm{~A}->135 \mathrm{~A} & 0.52940 \\ 129 \mathrm{~A}->138 \mathrm{~A} & 0.22517 \\ 129 \mathrm{~A}->140 \mathrm{~A} & -0.21226 \\ 129 \mathrm{~A}->141 \mathrm{~A} & 0.22475 \\ 119 \mathrm{~B}->128 \mathrm{~B} & 0.11115 \\ 119 \mathrm{~B}->129 \mathrm{~B} & -0.31282 \\ 124 \mathrm{~B}->128 \mathrm{~B} & -0.11658 \\ 127 \mathrm{~B}->134 \mathrm{~B} & 0.20309 \\ 127 \mathrm{~B}->142 \mathrm{~B} & -0.12115\end{array}$

Table S7. Standard orientation of the optimized geometry for the closed-shell quinoidal form of the ring-opening form of 1.

\begin{tabular}{ccccc}
\hline \multirow{2}{*}{ Tag } & Symbol & $\mathrm{X}$ & Coordinates (Angstroms) & \\
& & $\mathrm{Y}$ & $\mathrm{Y}$ & $\mathrm{Z}$ \\
\hline
\end{tabular}




\begin{tabular}{|c|c|c|c|c|}
\hline 1 & $\mathrm{C}$ & -2.2144140 & 5.2375090 & -0.3015120 \\
\hline 2 & $\mathrm{C}$ & -0.1348070 & 3.4324320 & 0.3139820 \\
\hline 3 & $\mathrm{C}$ & -0.9827840 & 5.7170810 & 0.2054930 \\
\hline 4 & $\mathrm{C}$ & -2.4291010 & 3.8964380 & -0.4328250 \\
\hline 5 & $\mathrm{C}$ & -1.4357050 & 2.9477020 & -0.0580100 \\
\hline 6 & $\mathrm{C}$ & 0.0162420 & 4.8367610 & 0.5097770 \\
\hline 7 & $\mathrm{C}$ & 1.0349820 & 2.6114630 & 0.3797900 \\
\hline 8 & $\mathrm{C}$ & 3.4620200 & 1.0779320 & 0.4525630 \\
\hline 9 & $\mathrm{C}$ & 1.2276460 & 1.4965700 & -0.4742910 \\
\hline 10 & $\mathrm{C}$ & 2.1219250 & 2.9580350 & 1.2566460 \\
\hline 11 & $\mathrm{C}$ & 3.2528590 & 2.2208370 & 1.3097830 \\
\hline 12 & $\mathrm{C}$ & 2.3764120 & 0.7593450 & -0.4596730 \\
\hline 13 & $\mathrm{~S}$ & 2.5173360 & -0.5920210 & -1.5611590 \\
\hline 14 & $\mathrm{C}$ & 4.1203690 & -1.2007090 & -1.2104070 \\
\hline 15 & $\mathrm{C}$ & 4.9370550 & -0.6282120 & -0.2144020 \\
\hline 16 & $\mathrm{~N}$ & 4.6030620 & 0.4559110 & 0.5635180 \\
\hline 17 & $\mathrm{C}$ & 6.2073140 & -1.1917120 & 0.0112230 \\
\hline 18 & $\mathrm{C}$ & 6.6496420 & -2.2751930 & -0.7168590 \\
\hline 19 & $\mathrm{C}$ & 5.8273160 & -2.8293400 & -1.7028740 \\
\hline 20 & $\mathrm{C}$ & 4.5744600 & -2.2957910 & -1.9481780 \\
\hline 21 & $\mathrm{C}$ & -1.8343890 & 1.5898500 & 0.0509120 \\
\hline 22 & $\mathrm{~N}$ & -3.0124220 & 1.1408510 & -0.4875380 \\
\hline 23 & $\mathrm{C}$ & -3.0581440 & -0.1404440 & -0.1683270 \\
\hline 24 & $\mathrm{C}$ & -1.8854750 & -0.4563280 & 0.6417280 \\
\hline 25 & $\mathrm{~N}$ & -1.1488410 & 0.6366420 & 0.7485220 \\
\hline 26 & $\mathrm{C}$ & -4.1503800 & -0.9944300 & -0.6535540 \\
\hline 27 & $\mathrm{C}$ & -6.2543980 & -2.5396260 & -1.6720830 \\
\hline 28 & $\mathrm{C}$ & -3.9539140 & -2.3407380 & -0.9808100 \\
\hline 29 & $\mathrm{C}$ & -5.4157200 & -0.4318350 & -0.8614280 \\
\hline 30 & $\mathrm{C}$ & -6.4579580 & -1.1981550 & -1.3608010 \\
\hline 31 & $\mathrm{C}$ & -4.9981640 & -3.1054200 & -1.4847290 \\
\hline 32 & $\mathrm{C}$ & -1.4996210 & -1.6865400 & 1.3436500 \\
\hline 33 & $\mathrm{C}$ & -0.6821340 & -3.9675400 & 2.7548670 \\
\hline 34 & $\mathrm{C}$ & -0.1409290 & -1.9378000 & 1.5759790 \\
\hline 35 & $\mathrm{C}$ & -2.4417420 & -2.5919210 & 1.8466070 \\
\hline 36 & $\mathrm{C}$ & -2.0347870 & -3.7212710 & 2.5441440 \\
\hline 37 & $\mathrm{C}$ & 0.2629700 & -3.0683340 & 2.2706560 \\
\hline 38 & $\mathrm{H}$ & -2.9975610 & 5.9408940 & -0.5669660 \\
\hline 39 & $\mathrm{H}$ & -0.8195780 & 6.7840340 & 0.3166480 \\
\hline
\end{tabular}




\begin{tabular}{lllll}
40 & $\mathrm{H}$ & -3.3803750 & 3.5041310 & -0.7708250 \\
41 & $\mathrm{H}$ & 0.9830820 & 5.2229690 & 0.8113210 \\
43 & $\mathrm{H}$ & 0.4429860 & 1.2440290 & -1.1761850 \\
44 & $\mathrm{H}$ & 1.9969840 & 3.7886230 & 1.9410240 \\
45 & $\mathrm{H}$ & 4.0545830 & 2.4484890 & 2.0030080 \\
46 & $\mathrm{H}$ & 6.8172020 & -0.7328870 & 0.7814010 \\
47 & $\mathrm{H}$ & 7.6315030 & -2.6955390 & -0.5270780 \\
48 & $\mathrm{H}$ & 6.1679420 & -3.6819210 & -2.2812420 \\
49 & $\mathrm{H}$ & 3.9366420 & -2.7276400 & -2.7135820 \\
50 & $\mathrm{H}$ & -7.0704670 & -3.1395200 & -2.0633540 \\
51 & $\mathrm{H}$ & -2.9737480 & -2.7863800 & -0.8529560 \\
52 & $\mathrm{H}$ & -5.5598440 & 0.6166630 & -0.6261400 \\
53 & $\mathrm{H}$ & -7.4348450 & -0.7477100 & -1.5089930 \\
54 & $\mathrm{H}$ & -4.8264680 & -4.1470850 & -1.7386590 \\
55 & $\mathrm{H}$ & -0.3664580 & -4.8528660 & 3.2984570 \\
56 & $\mathrm{H}$ & 0.5871660 & -1.2252430 & 1.2049860 \\
57 & $\mathrm{H}$ & -3.4992560 & -2.4005130 & 1.7053930 \\
58 & $\mathrm{H}$ & -2.7802980 & -4.4083840 & 2.9328810 \\
\hline
\end{tabular}

SCF Done: $\quad$ E(RCAM-B3LYP)

$=-1831.73257639 \quad$ A.U.

Zero-point correction

$=0.454421($ Hartree/Particle $)$

Thermal correction to Energy

$=0.481997$

Thermal correction to Enthalpy

$=0.482941$

Thermal correction to Gibbs Free Energy

$=0.393359$

Sum of electronic and zero-point Energies

$=-1831.288393$

Sum of electronic and thermal Energies

$=-1831.260817$

Sum of electronic and thermal Enthalpies

$=-1831.259873$

Sum of electronic and thermal Free Energies $=-1831.349454$

$\begin{array}{lllllll}\text { Low frequencies --- } & -0.0005 & 0.0013 & 0.0014 & 0.4699 & 0.5765 & 1.4695\end{array}$

$\begin{array}{llll}\text { Low frequencies --- } & 13.2818 & 16.2794 & 28.0757\end{array}$

The Results of the TDDFT calculation

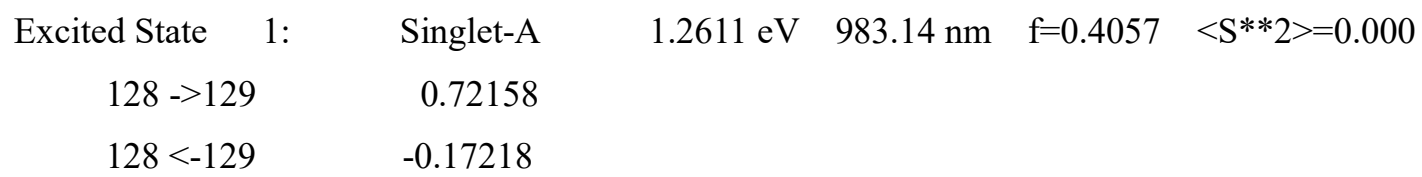


This state for optimization and/or second-order correction.

Total Energy, E(TD-HF/TD-DFT) = -1831.74390237

Copying the excited state density for this state as the 1-particle RhoCI density.

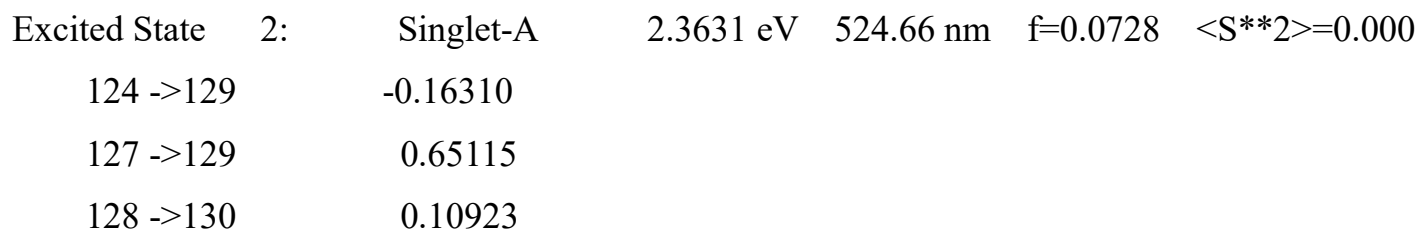

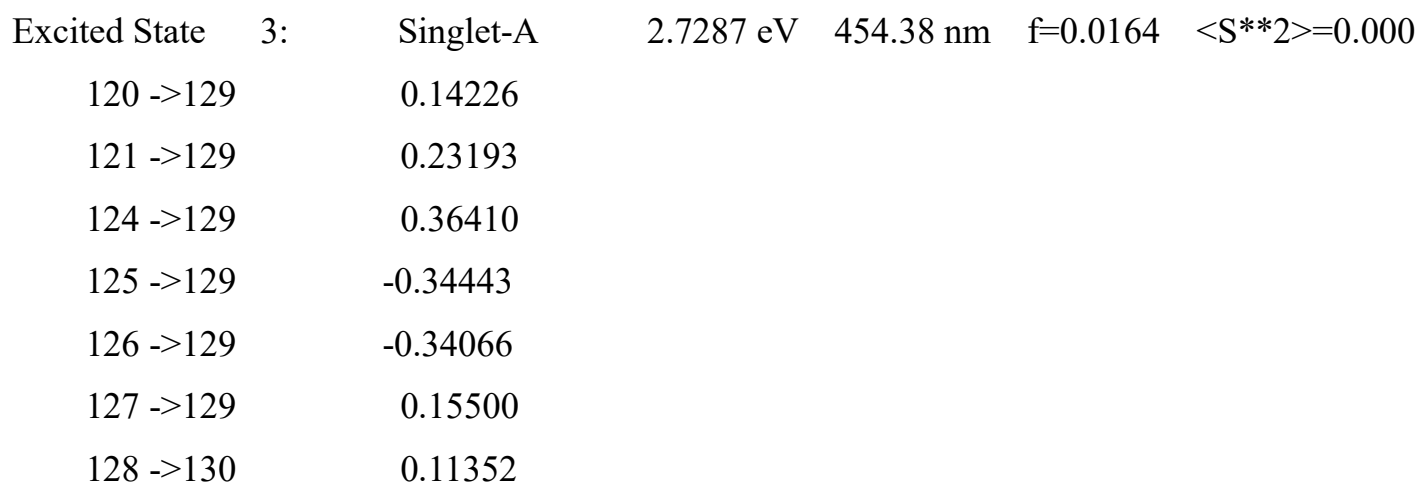

Excited State $\quad 4: \quad$ Singlet-A $\quad 2.8060 \mathrm{eV} \quad 441.86 \mathrm{~nm} \quad \mathrm{f}=0.0132 \quad<\mathrm{S}^{* *} 2>=0.000$

$\begin{array}{cc}121->129 & 0.22108 \\ 124->129 & 0.10870 \\ 125->129 & -0.16814 \\ 126->129 & 0.52473 \\ 128->130 & 0.30383\end{array}$

Excited State $\quad 5: \quad$ Singlet-A $\quad 3.0840 \mathrm{eV} \quad 402.02 \mathrm{~nm} \quad \mathrm{f}=0.1520 \quad<\mathrm{S}^{* *} 2>=0.000$

$\begin{array}{cc}121->129 & -0.31823 \\ 122->129 & -0.28621 \\ 124->129 & 0.11284 \\ 125->129 & 0.14207 \\ 126->129 & -0.12341 \\ 128->130 & 0.47106\end{array}$

Excited State 6: $\quad$ Singlet-A $\quad 3.2599 \mathrm{eV} \quad 380.33 \mathrm{~nm} \quad \mathrm{f}=0.2852 \quad<\mathrm{S}^{* *} 2>=0.000$

$\begin{array}{rr}122->129 & 0.57530 \\ 127->129 & -0.10001 \\ 128->130 & 0.29905 \\ 128->131 & -0.12255\end{array}$




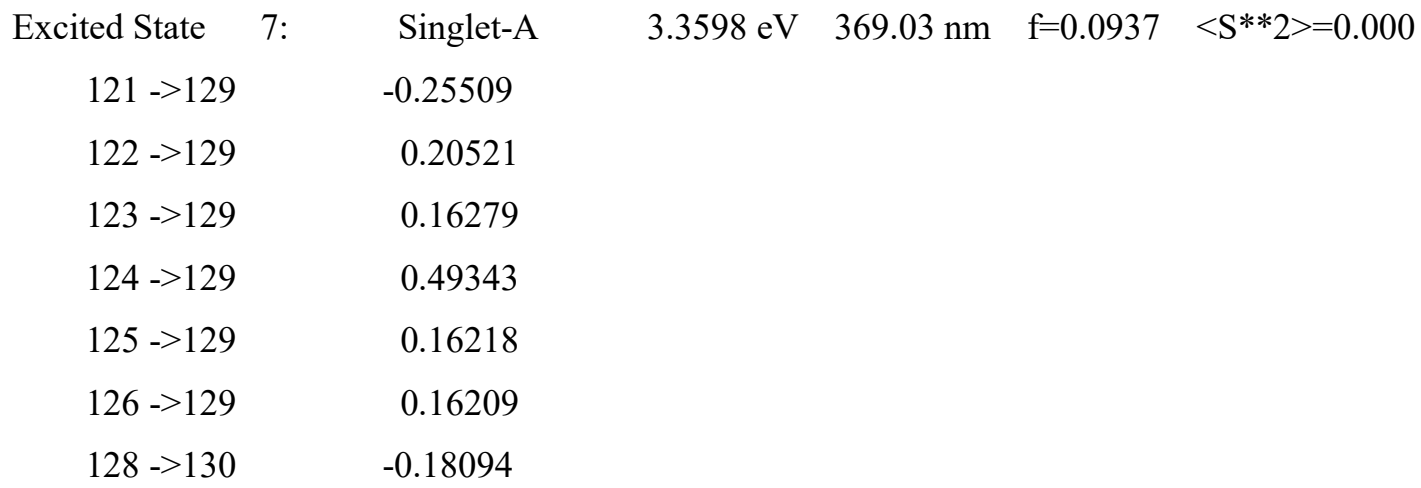

$\begin{array}{ll}116->129 & 0.11260 \\ 121->129 & 0.40036 \\ 123->129 & 0.29439 \\ 125->129 & 0.44817\end{array}$

Excited State $\quad 10: \quad$ Singlet-A $\quad 3.6739 \mathrm{eV} \quad 337.48 \mathrm{~nm} \quad \mathrm{f}=0.0176 \quad<\mathrm{S}^{* * 2}>=0.000$

$\begin{array}{cc}115->129 & 0.13966 \\ 119->129 & -0.11701 \\ 120->129 & -0.42827 \\ 123->129 & 0.43074 \\ 125->129 & -0.20940 \\ 128->131 & -0.10392\end{array}$

Excited State 11: $\quad$ Singlet-A $\quad 3.7581 \mathrm{eV} \quad 329.91 \mathrm{~nm} \quad \mathrm{f}=0.2345 \quad<\mathrm{S} * * 2>=0.000$

$\begin{array}{lr}120->129 & 0.25195 \\ 123->129 & 0.31864 \\ 124->129 & -0.17266 \\ 125->129 & -0.10210 \\ 128->131 & 0.48623\end{array}$

Excited State $\quad 12: \quad$ Singlet-A $\quad 3.7946 \mathrm{eV} \quad 326.74 \mathrm{~nm} \quad \mathrm{f}=0.1676 \quad<\mathrm{S} * * 2>=0.000$
$117->129$
$-0.13124$
$120->129$
$-0.36883$
$121->129$
0.13323 


$\begin{array}{cc}123->129 & -0.26638 \\ 125->129 & 0.15941 \\ 126->129 & -0.14402 \\ 128->131 & 0.40540\end{array}$

Excited State 13: $\quad$ Singlet-A $\quad 3.9914 \mathrm{eV} \quad 310.63 \mathrm{~nm} \quad \mathrm{f}=0.0051 \quad<\mathrm{S} * * 2>=0.000$

$\begin{array}{cc}115->129 & 0.22747 \\ 117->129 & 0.46265 \\ 118->129 & 0.22197 \\ 119->129 & 0.28558 \\ 120->129 & -0.10306 \\ 122->129 & 0.10100 \\ 128->132 & 0.16607\end{array}$

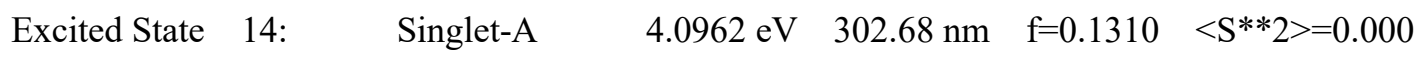

$$
\begin{array}{lr}
128->132 & -0.10582 \\
128->133 & 0.66383 \\
128->135 & 0.11092
\end{array}
$$
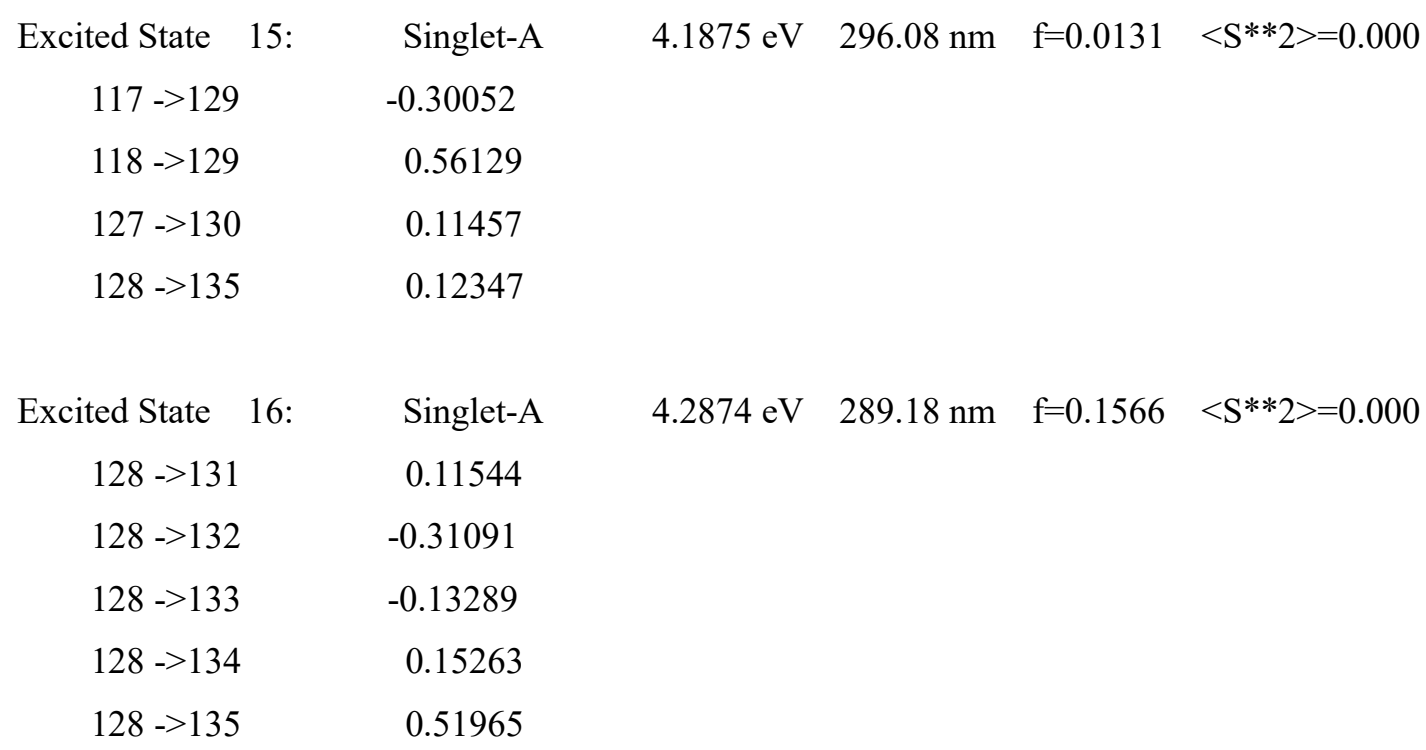

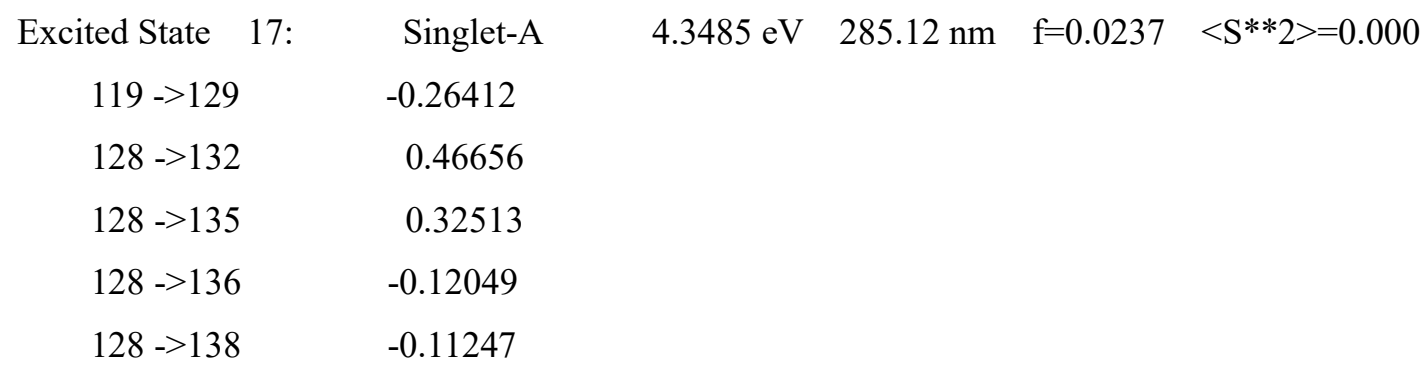



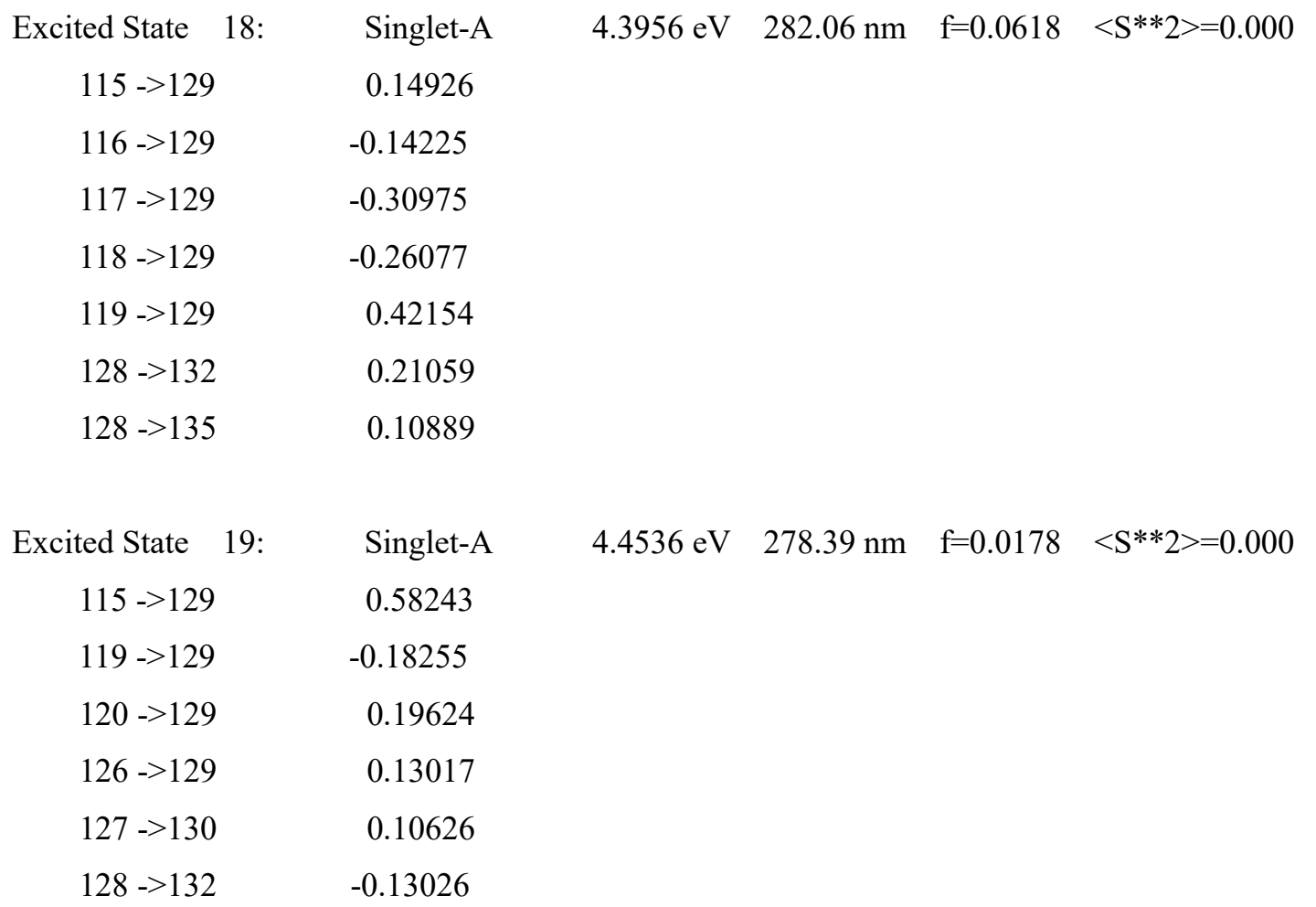

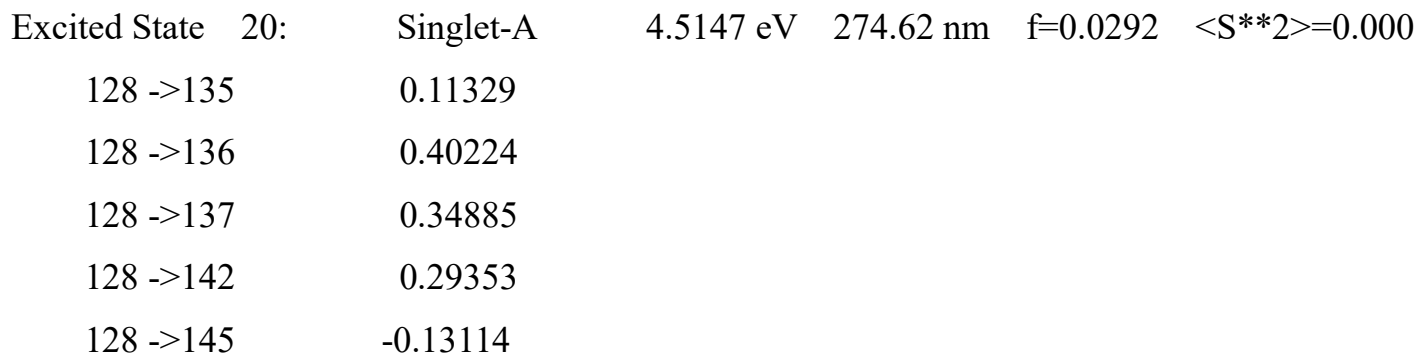




\section{Singlet biradical}
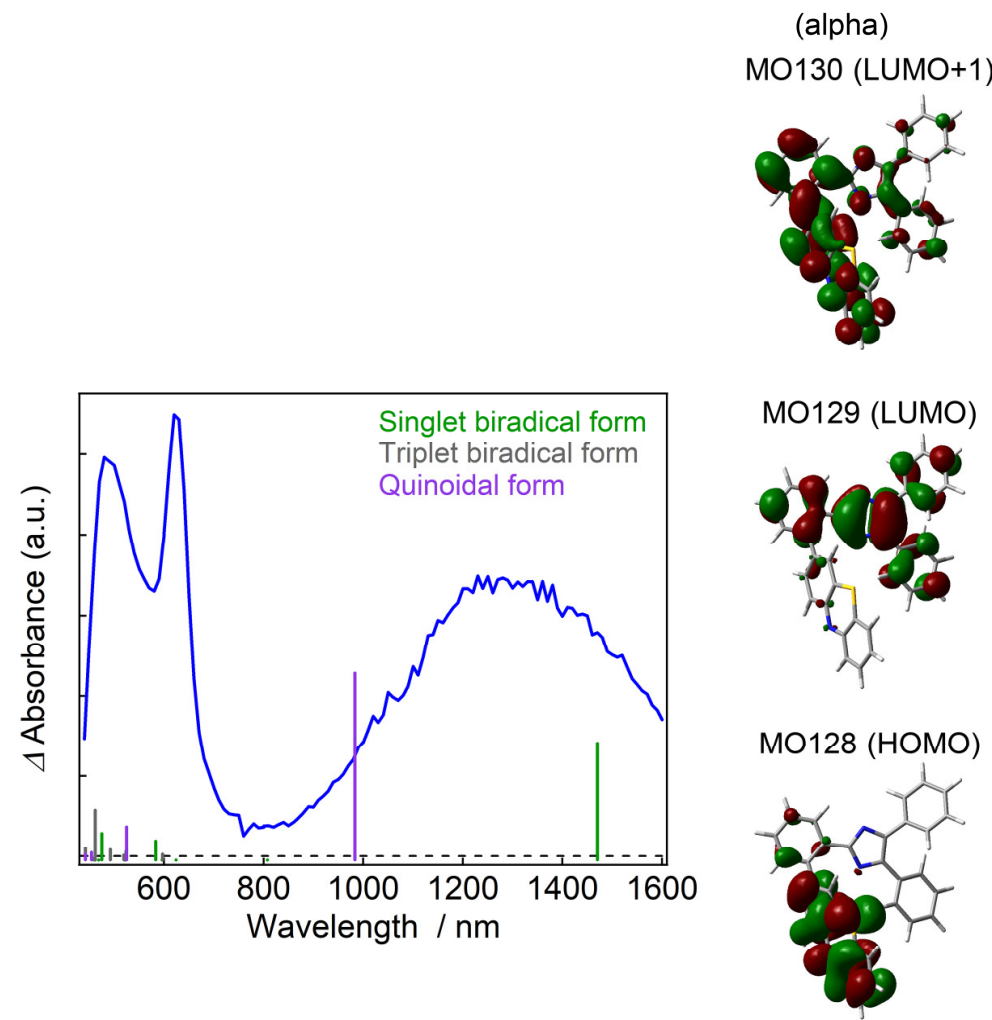

MO127 (HOMO-1)

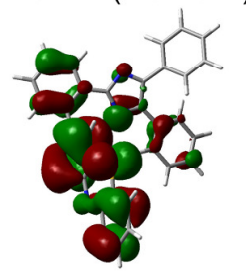

(beta)

MO130 (LUMO+1) MO130 (LUMO+1)

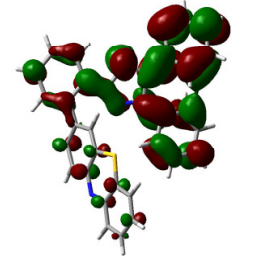

MO129 (LUMO)

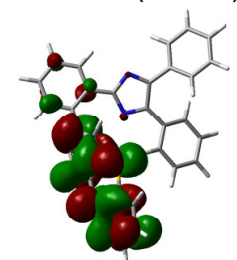

MO128 (HOMO)

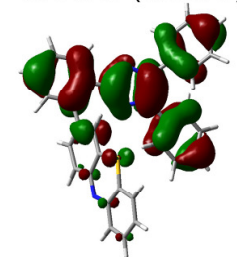

MO127 (HOMO-1)

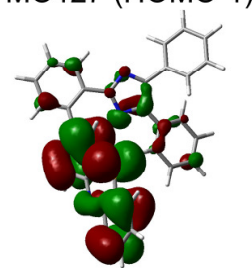

Quinoidal

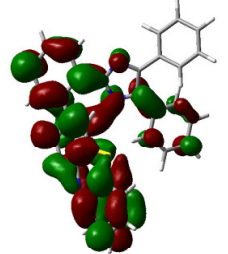

MO129 (LUMO)

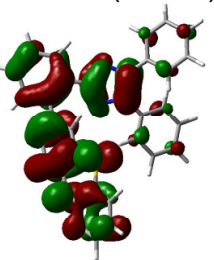

MO128 (HOMO)

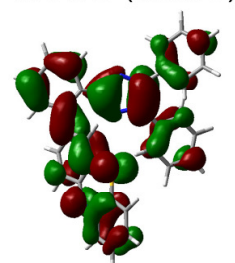

MO127 (HOMO-1)

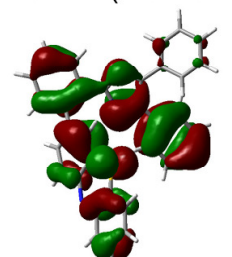

Figure S50. (a) UV-vis absorption spectrum of 1 in benzene at room temperature. The calculated absorption spectrum ((U)CAM-B3LYP/6-31G+(d)// (U)CAM-B3LYP /6-31G(d) level of the theory) is shown by the blue vertical lines. (b) The relevant molecular orbitals of the 1 calculated at the (U)CAM-B3LYP /6-31G(d) level of the theory. 
Table S8. Standard orientation of the optimized geometry for the closed form of 2.

\begin{tabular}{|c|c|c|c|c|}
\hline \multirow{2}{*}{ Tag } & \multirow{2}{*}{ Symbol } & \multicolumn{3}{|c|}{ Coordinates (Angstroms) } \\
\hline & & $\mathrm{X}$ & $\mathrm{Y}$ & Z \\
\hline 1 & $\mathrm{C}$ & -1.2675830 & 5.5618270 & -0.1062890 \\
\hline 2 & $\mathrm{C}$ & 0.0819500 & 5.3888870 & 0.2054530 \\
\hline 3 & $\mathrm{C}$ & 0.6223320 & 4.1097640 & 0.3487570 \\
\hline 4 & $\mathrm{C}$ & -0.2175950 & 3.0249870 & 0.1773800 \\
\hline 5 & $\mathrm{C}$ & -1.5706440 & 3.1966230 & -0.1392260 \\
\hline 6 & $\mathrm{C}$ & -2.1115920 & 4.4671830 & -0.2830400 \\
\hline 7 & $\mathrm{C}$ & -2.1561750 & 1.8653260 & -0.2416200 \\
\hline 8 & $\mathrm{C}$ & 0.1448180 & 1.5364220 & 0.2850170 \\
\hline 9 & $\mathrm{C}$ & 0.5128090 & 1.1743150 & 1.7001270 \\
\hline 10 & $\mathrm{C}$ & 1.6569750 & 0.5707000 & 2.0271210 \\
\hline 11 & $\mathrm{C}$ & 2.6477380 & 0.2017030 & 1.0213730 \\
\hline 12 & $\mathrm{C}$ & 2.3306450 & 0.5556010 & -0.3705920 \\
\hline 13 & $\mathrm{C}$ & 1.1949990 & 1.1667550 & -0.7191740 \\
\hline 14 & $\mathrm{~N}$ & -1.1686800 & 0.9475340 & -0.0426290 \\
\hline 15 & $\mathrm{C}$ & -1.7548860 & -0.2966530 & -0.0301570 \\
\hline 16 & $\mathrm{C}$ & -3.1016690 & -0.0411950 & -0.2700390 \\
\hline 17 & $\mathrm{~N}$ & -3.3318430 & 1.3143480 & -0.3923270 \\
\hline 18 & $\mathrm{~N}$ & 3.7272020 & -0.3970560 & 1.3828360 \\
\hline 19 & $\mathrm{C}$ & 4.6340030 & -0.7256780 & 0.3713270 \\
\hline 20 & $\mathrm{C}$ & 4.3883690 & -0.4178190 & -0.9709050 \\
\hline 21 & $\mathrm{C}$ & 5.8232300 & -1.3779590 & 0.7012690 \\
\hline 22 & $\mathrm{C}$ & 6.7405440 & -1.7138900 & -0.2843700 \\
\hline 23 & $\mathrm{C}$ & 6.4735360 & -1.3965380 & -1.6167020 \\
\hline 24 & $\mathrm{C}$ & 5.2951630 & -0.7460610 & -1.9670910 \\
\hline 25 & $\mathrm{C}$ & -1.0012100 & -1.5241510 & 0.2854900 \\
\hline 26 & $\mathrm{C}$ & -4.2123740 & -0.9979970 & -0.3972680 \\
\hline 27 & $\mathrm{C}$ & 0.0668420 & -1.9422420 & -0.5135720 \\
\hline 28 & $\mathrm{C}$ & 0.8073100 & -3.0673070 & -0.1652360 \\
\hline 29 & $\mathrm{C}$ & 0.4736300 & -3.7976820 & 0.9718200 \\
\hline 30 & $\mathrm{C}$ & -0.6071310 & -3.4032820 & 1.7567190 \\
\hline 31 & $\mathrm{C}$ & -1.3379030 & -2.2700510 & 1.4195540 \\
\hline 32 & $\mathrm{C}$ & -5.5205370 & -0.5475150 & -0.1891470 \\
\hline 33 & $\mathrm{C}$ & -6.5940800 & -1.4219640 & -0.3023290 \\
\hline 34 & $\mathrm{C}$ & -6.3800370 & -2.7592530 & -0.6264890 \\
\hline 35 & $\mathrm{C}$ & -5.0822110 & -3.2116320 & -0.8467760 \\
\hline 36 & $\mathrm{C}$ & -4.0052960 & -2.3387560 & -0.7392250 \\
\hline
\end{tabular}




\begin{tabular}{lllll}
37 & $\mathrm{H}$ & -1.6645880 & 6.5630900 & -0.2138150 \\
38 & $\mathrm{H}$ & 0.7163370 & 6.2559280 & 0.3367210 \\
39 & $\mathrm{H}$ & 1.6691550 & 3.9654810 & 0.5880540 \\
40 & $\mathrm{H}$ & -3.1578820 & 4.5939570 & -0.5263920 \\
41 & $\mathrm{H}$ & -0.2261000 & 1.4300420 & 2.4503110 \\
42 & $\mathrm{H}$ & 1.9012640 & 0.3125110 & 3.0489300 \\
43 & $\mathrm{H}$ & 0.9984840 & 1.4034390 & -1.7570430 \\
44 & $\mathrm{H}$ & 5.9947680 & -1.6065790 & 1.7449090 \\
45 & $\mathrm{H}$ & 7.6595710 & -2.2192910 & -0.0200760 \\
46 & $\mathrm{H}$ & 7.1855140 & -1.6561600 & -2.3892650 \\
47 & $\mathrm{H}$ & 5.0646960 & -0.4885760 & -2.9921230 \\
48 & $\mathrm{H}$ & 0.3121820 & -1.3854690 & -1.4080270 \\
49 & $\mathrm{H}$ & 1.6396450 & -3.3757960 & -0.7854410 \\
50 & $\mathrm{H}$ & 1.0496850 & -4.6734580 & 1.2421320 \\
51 & $\mathrm{H}$ & -0.8748950 & -3.9726330 & 2.6376850 \\
52 & $\mathrm{H}$ & -2.1736550 & -1.9503730 & 2.0294280 \\
53 & $\mathrm{H}$ & -5.6760560 & 0.4944440 & 0.0559420 \\
54 & $\mathrm{H}$ & -7.6007030 & -1.0585510 & -0.1363790 \\
55 & $\mathrm{H}$ & -7.2170300 & -3.4406360 & -0.7135430 \\
56 & $\mathrm{H}$ & -4.9058020 & -4.2461150 & -1.1142190 \\
57 & $\mathrm{H}$ & -3.0032200 & -2.6964460 & -0.9354820 \\
58 & $\mathrm{O}$ & 3.2347580 & 0.2203240 & -1.3413040 \\
\hline
\end{tabular}

$\begin{array}{llll}\text { SCF Done: E }(\mathrm{RM} 052 \mathrm{X}) & = & -1509.51213088 \quad \text { A.U. } \\ & = & 0.461220 \text { (Hartree/Particle) } \\ \text { Zero-point correction } & = & 0.487856 \\ \text { Thermal correction to Energy } & = & 0.488800 \\ \text { Thermal correction to Enthalpy } & = & 0.400442 \\ \text { Thermal correction to Gibbs Free Energy } & = & -1509.050911 \\ \text { Sum of electronic and zero-point Energies } & = & -1509.024275 \\ \text { Sum of electronic and thermal Energies } & = & -1509.023331 \\ \text { Sum of electronic and thermal Enthalpies } & = & -1509.111689 \\ \text { Sum of electronic and thermal Free Energies } & = & \\ & & & \\ \text { Low frequencies --- } \quad-9.9824 \quad-6.1618 & -0.0007 & 0.0003 & 0.0007 \\ \text { Low frequencies --- } 11.3331 \quad 18.0400 & 31.5859 & \end{array}$


The Result for the TDDFT calculation

Excited State 1: $\quad$ Singlet-A $\quad 2.6592 \mathrm{eV} \quad 466.25 \mathrm{~nm} \quad \mathrm{f}=0.0005 \quad<\mathrm{S} * * 2>=0.000$ $124->125 \quad 0.70376$

This state for optimization and/or second-order correction.

Total Energy, E(TD-HF/TD-KS) = - 1509.23197292

Copying the excited state density for this state as the 1-particle RhoCI density.

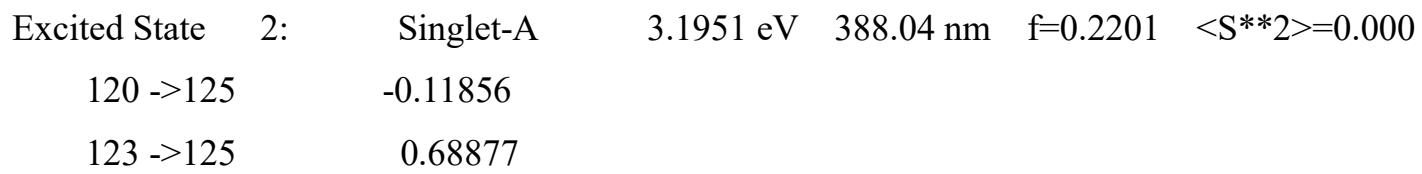
Excited State $\quad 3: \quad$ Singlet-A $\quad 3.9043 \mathrm{eV} \quad 317.55 \mathrm{~nm} \quad \mathrm{f}=0.2229 \quad<\mathrm{S} * * 2>=0.000$

$124->126 \quad 0.68187$
$124->127 \quad-0.13587$
Excited State $\quad 4: \quad$ Singlet-A $\quad 4.0482 \mathrm{eV} \quad 306.27 \mathrm{~nm} \quad \mathrm{f}=0.0015 \quad<\mathrm{S}^{* *} 2>=0.000$
$115->125 \quad 0.12646$
$116->125 \quad 0.10431$
$119->125 \quad-0.13956$
$121->125 \quad 0.41065$
$122 \rightarrow 125 \quad 0.52315$
Excited State 5: $\quad$ Singlet-A $\quad 4.1242 \mathrm{eV} \quad 300.63 \mathrm{~nm} \quad \mathrm{f}=0.4358 \quad<\mathrm{S}^{* *} 2>=0.000$ $120->125 \quad 0.29696$
$121->125 \quad-0.17299$
$124->127 \quad 0.56734$
$124->128 \quad-0.15129$

Excited State 6: $\quad$ Singlet-A $\quad 4.1327 \mathrm{eV} \quad 300.01 \mathrm{~nm} \quad \mathrm{f}=0.0520 \quad<\mathrm{S} * * 2>=0.000$

$\begin{array}{lc}115->125 & 0.36931 \\ 116->125 & 0.12146 \\ 118->125 & 0.11996 \\ 120->125 & 0.31749 \\ 121->125 & -0.30503 \\ 122->125 & 0.11787 \\ 124->127 & -0.30482\end{array}$

Excited State $\quad 7: \quad$ Singlet-A $\quad 4.1480 \mathrm{eV} \quad 298.90 \mathrm{~nm} \quad \mathrm{f}=0.0271 \quad<\mathrm{S}^{* *} 2>=0.000$ 


$\begin{array}{cc}115->125 & 0.51651 \\ 116->125 & 0.16596 \\ 120->125 & -0.25453 \\ 121->125 & 0.16967 \\ 122->125 & -0.27404 \\ 124->127 & 0.12694\end{array}$

Excited State $\quad 8: \quad$ Singlet-A $\quad 4.1833 \mathrm{eV} \quad 296.38 \mathrm{~nm} \quad \mathrm{f}=0.0657 \quad<\mathrm{S}^{* *} 2>=0.000$ $118->125 \quad-0.21512$

$120->125 \quad 0.43355$

$121->125 \quad 0.36002$

$122->125 \quad-0.29178$

$123->129 \quad 0.10237$

$124->128 \quad 0.12432$

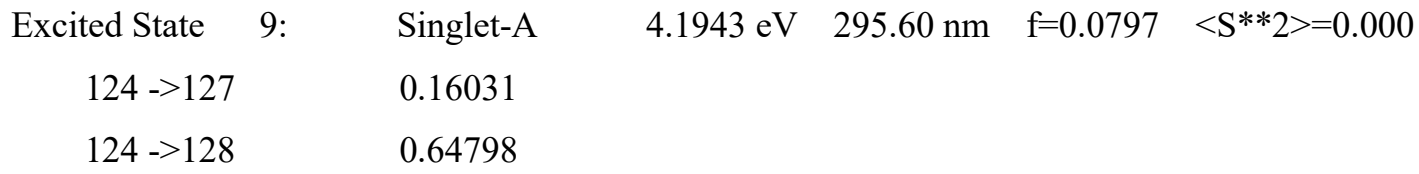

Excited State $\quad 10: \quad$ Singlet-A $\quad 4.2738 \mathrm{eV} \quad 290.11 \mathrm{~nm} \quad \mathrm{f}=0.0089 \quad<\mathrm{S} * * 2>=0.000$

$\begin{array}{ll}117->125 & 0.10877 \\ 118->125 & 0.19670 \\ 119->125 & 0.64959 \\ 122->125 & 0.10394\end{array}$

Excited State 11: $\quad$ Singlet-A $\quad 4.3476 \mathrm{eV} \quad 285.18 \mathrm{~nm} \quad \mathrm{f}=0.0054 \quad<\mathrm{S}^{* *} 2>=0.000$

$\begin{array}{cc}117->125 & 0.14119 \\ 118->125 & 0.58523 \\ 119->125 & -0.20300 \\ 121->125 & 0.16196 \\ 122->125 & -0.17680\end{array}$

Excited State $\quad 12: \quad$ Singlet-A $\quad 4.4094 \mathrm{eV} \quad 281.18 \mathrm{~nm} \quad \mathrm{f}=0.0078 \quad<\mathrm{S}^{* * 2} 2=0.000$ $123->126 \quad-0.12399$

$124->129 \quad 0.21829$

$124->130 \quad 0.63635$

Excited State 13: $\quad$ Singlet-A $\quad 4.4534 \mathrm{eV} \quad 278.41 \mathrm{~nm} \quad \mathrm{f}=0.0114 \quad<\mathrm{S} * * 2>=0.000$ $117->125 \quad 0.61301$ 


$\begin{array}{ll}118->125 & -0.19702 \\ 123->126 & -0.15771 \\ 123->129 & -0.12799\end{array}$

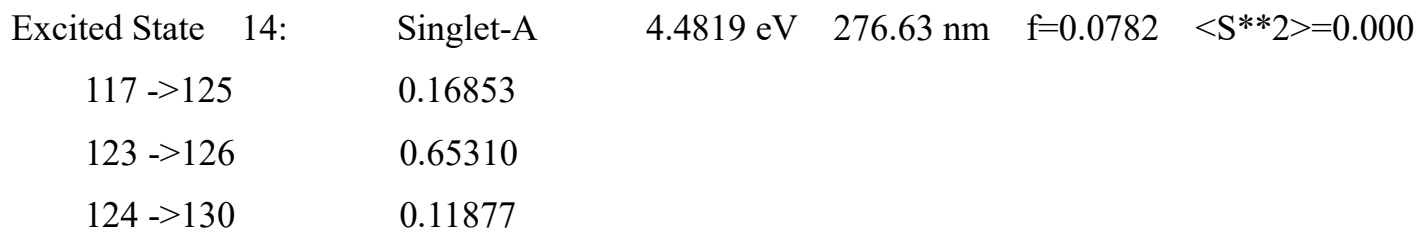

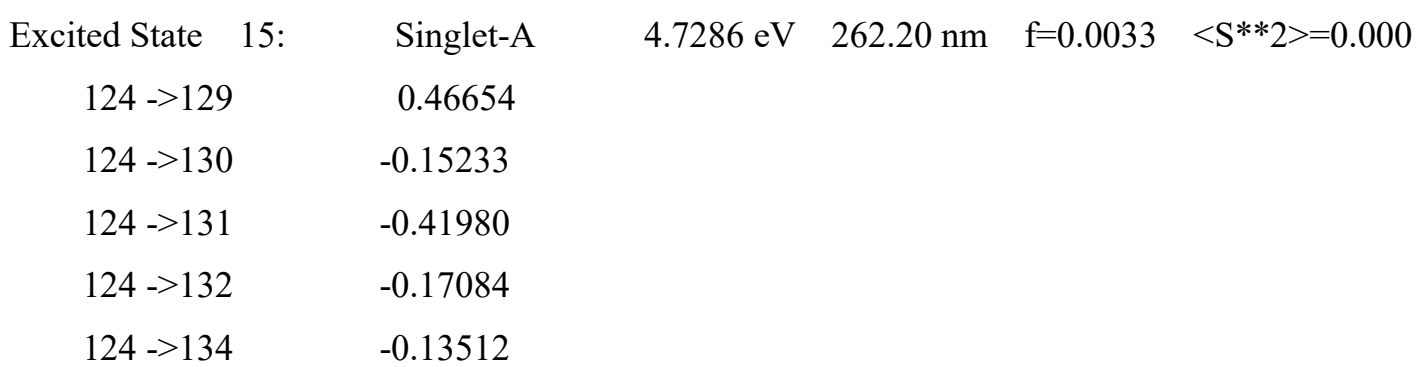

Excited State 16: $\quad$ Singlet-A $\quad 4.7614 \mathrm{eV} \quad 260.39 \mathrm{~nm} \quad \mathrm{f}=0.1462 \quad<$ S**2 $>=0.000$

$\begin{array}{lc}114->125 & -0.17512 \\ 116->125 & -0.29746 \\ 117->125 & -0.14155 \\ 123->128 & 0.48989 \\ 124->129 & 0.18713 \\ 124->132 & -0.11010\end{array}$

Excited State 17: $\quad$ Singlet-A $\quad 4.8014 \mathrm{eV} \quad 258.23 \mathrm{~nm} \quad \mathrm{f}=0.0056 \quad<\mathrm{S}^{* * 2} 2=0.000$

$\begin{array}{cc}123->127 & 0.12822 \\ 123->128 & -0.22397 \\ 124->129 & 0.39860 \\ 124->130 & -0.15598 \\ 124->131 & 0.45696 \\ 124->134 & 0.13194\end{array}$

Excited State 18: $\quad$ Singlet-A $\quad 4.8240 \mathrm{eV} \quad 257.01 \mathrm{~nm} \quad \mathrm{f}=0.0096 \quad<\mathrm{S} * * 2>=0.000$

$\begin{array}{ll}123->127 & 0.62086 \\ 124->129 & -0.12098 \\ 124->132 & -0.24214\end{array}$

Excited State 19: $\quad$ Singlet-A $\quad 4.8349 \mathrm{eV} \quad 256.44 \mathrm{~nm} \quad \mathrm{f}=0.0127 \quad<\mathrm{S}^{* *} 2>=0.000$ $114->125 \quad 0.21619$ 


$\begin{array}{ll}115->125 & -0.10985 \\ 116->125 & 0.38898 \\ 117->125 & 0.10214 \\ 123 \text {->127 } & 0.15415 \\ 123 \text {->128 } & 0.37984 \\ 123 \text {->129 } & 0.18661 \\ 124->132 & 0.20222\end{array}$

\begin{tabular}{|c|c|}
\hline Excited State 20: & Singlet- \\
\hline $114->125$ & -0.10216 \\
\hline $116->125$ & -0.19922 \\
\hline $122->127$ & 0.11118 \\
\hline $123->127$ & 0.16929 \\
\hline $124->131$ & -0.14741 \\
\hline $124->132$ & 0.45988 \\
\hline $124->133$ & -0.12024 \\
\hline $124->135$ & 0.28481 \\
\hline
\end{tabular}

Excited State 21: $\quad$ Singlet-A $\quad 4.9118 \mathrm{eV} \quad 252.42 \mathrm{~nm} \quad \mathrm{f}=0.0349 \quad<\mathrm{S}^{* *} 2>=0.000$

$\begin{array}{lr}122->127 & 0.21883 \\ 122->132 & -0.12646 \\ 123->127 & -0.11643 \\ 124->132 & -0.32344 \\ 124->133 & -0.13390 \\ 124->134 & 0.15053 \\ 124->135 & 0.45104 \\ 124->136 & -0.11397\end{array}$

Excited State 22: $\quad$ Singlet-A $\quad 5.0205 \mathrm{eV} \quad 246.95 \mathrm{~nm} \quad \mathrm{f}=0.1270 \quad<\mathrm{S} * * 2>=0.000$

$\begin{array}{cc}114->125 & 0.17571 \\ 115->125 & 0.12883 \\ 116->125 & -0.32806 \\ 117->125 & 0.12020 \\ 123->129 & 0.49771 \\ 123->131 & 0.14222\end{array}$

Excited State 23: $\quad$ Singlet-A $\quad 5.0292 \mathrm{eV} \quad 246.53 \mathrm{~nm} \quad \mathrm{f}=0.0014 \quad<\mathrm{S}^{* *} 2>=0.000$

$122->127 \quad 0.10281$

$124->133 \quad 0.61157$ 


$\begin{array}{rr}124->136 & -0.19450 \\ 124->137 & 0.15339 \\ 124->139 & 0.15073\end{array}$

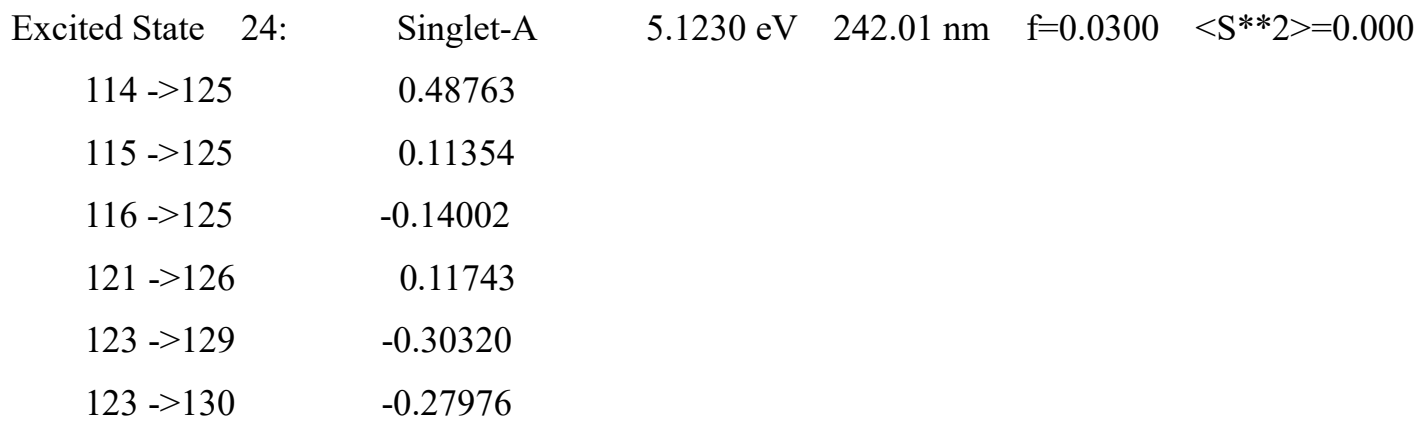

Excited State $\quad 25: \quad$ Singlet-A $\quad 5.1454 \mathrm{eV} \quad 240.96 \mathrm{~nm} \quad \mathrm{f}=0.0044 \quad<\mathrm{S}^{* *} 2>=0.000$

$\begin{array}{ll}114->125 & 0.26020 \\ 122->126 & 0.11896 \\ 123->130 & 0.58253 \\ 123->131 & -0.12163\end{array}$

Excited State 26: $\quad$ Singlet-A $\quad 5.2177 \mathrm{eV} \quad 237.62 \mathrm{~nm} \quad \mathrm{f}=0.0011 \quad<\mathrm{S} * * 2>=0.000$

$\begin{array}{cc}113->125 & 0.13081 \\ 122->126 & -0.11505 \\ 124->131 & -0.22309 \\ 124->134 & 0.57511 \\ 124->137 & -0.10841\end{array}$

Excited State $\quad 27: \quad$ Singlet-A $\quad 5.2187 \mathrm{eV} \quad 237.58 \mathrm{~nm} \quad \mathrm{f}=0.1734 \quad<\mathrm{S}^{* *} 2>=0.000$

$\begin{array}{lr}112->125 & -0.16365 \\ 121->126 & 0.30243 \\ 122->126 & 0.43681 \\ 123->131 & 0.31235 \\ 124->134 & 0.18871\end{array}$

Excited State 28: $\quad$ Singlet-A $\quad 5.2518 \mathrm{eV} \quad 236.08 \mathrm{~nm} \quad \mathrm{f}=0.1082 \quad<$ S $* * 2>=0.000$

$\begin{array}{cc}111->125 & 0.11651 \\ 112->125 & -0.25047 \\ 114->125 & 0.13371 \\ 121->126 & -0.26379 \\ 122->126 & -0.22093 \\ 123->129 & -0.12050\end{array}$




$$
\begin{array}{ll}
123->130 & 0.10980 \\
123->131 & 0.41879
\end{array}
$$

Excited State 29: $\quad$ Singlet-A $\quad 5.2960 \mathrm{eV} \quad 234.11 \mathrm{~nm} \quad \mathrm{f}=0.0238 \quad<\mathrm{S} * * 2>=0.000$

$\begin{array}{cc}117->126 & -0.15892 \\ 120->126 & -0.26611 \\ 121->126 & 0.35664 \\ 121->127 & -0.24169 \\ 122->126 & -0.32888 \\ 122->128 & -0.10660\end{array}$

Excited State $\quad 30: \quad$ Singlet-A $\quad 5.3145 \mathrm{eV} \quad 233.30 \mathrm{~nm} \quad \mathrm{f}=0.0065 \quad<$ S**2 $>=0.000$

$\begin{array}{lc}117->126 & 0.24421 \\ 120->126 & 0.31259 \\ 121->126 & 0.29518 \\ 121->128 & 0.22873 \\ 122->126 & -0.27582 \\ 122->127 & 0.14050 \\ 124->134 & 0.14322\end{array}$

Excited State $\quad 31: \quad$ Singlet-A $\quad 5.3451 \mathrm{eV} \quad 231.96 \mathrm{~nm} \quad \mathrm{f}=0.0036 \quad<\mathrm{S}^{* * 2}>=0.000$

$\begin{array}{ll}113->125 & 0.11838 \\ 124->135 & 0.10063 \\ 124->136 & 0.51291 \\ 124->137 & 0.39362 \\ 124->138 & -0.14593\end{array}$

Excited State $\quad 32: \quad$ Singlet-A $\quad 5.3714 \mathrm{eV} \quad 230.82 \mathrm{~nm} \quad \mathrm{f}=0.0072 \quad<\mathrm{S}^{* *} 2>=0.000$

$\begin{array}{lc}113->125 & 0.55775 \\ 123->131 & 0.10683 \\ 124->133 & -0.12691 \\ 124->135 & -0.13985 \\ 124->136 & -0.16498 \\ 124->137 & 0.17927 \\ 124->138 & 0.16821 \\ 124->139 & 0.14804\end{array}$

Excited State $\quad 33: \quad$ Singlet-A $\quad 5.3758 \mathrm{eV} \quad 230.63 \mathrm{~nm} \quad \mathrm{f}=0.0071 \quad<\mathrm{S}^{* *} 2>=0.000$ $113->125 \quad-0.34512$ 


$\begin{array}{cc}122->127 & 0.12592 \\ 124->133 & -0.17081 \\ 124->134 & 0.12328 \\ 124->135 & -0.22023 \\ 124->137 & 0.36519 \\ 124->138 & 0.18378 \\ 124->139 & 0.19134\end{array}$

\begin{tabular}{|c|c|}
\hline Excited State 34: & Singlet- \\
\hline $112->125$ & -0.10535 \\
\hline $118->126$ & -0.15175 \\
\hline $118->127$ & 0.17229 \\
\hline $118->128$ & -0.11094 \\
\hline $119->126$ & -0.17574 \\
\hline 119 ->130 & 0.18430 \\
\hline $120->126$ & -0.11087 \\
\hline $121->126$ & -0.13642 \\
\hline $122->126$ & 0.12262 \\
\hline $122->127$ & 0.32876 \\
\hline $123->130$ & 0.11857 \\
\hline $124->137$ & -0.23124 \\
\hline $124->138$ & -0.17603 \\
\hline
\end{tabular}

Excited State $35: \quad$ Singlet-A $\quad 5.4505 \mathrm{eV} \quad 227.47 \mathrm{~nm} \quad \mathrm{f}=0.0263 \quad<\mathrm{S}^{* *} 2>=0.000$

$\begin{array}{lr}114->126 & -0.14987 \\ 116->126 & 0.15313 \\ 118->127 & 0.10054 \\ 119->126 & 0.50700 \\ 119->130 & 0.11480 \\ 121->127 & -0.24361 \\ 123->132 & -0.12389\end{array}$

Excited State $36: \quad$ Singlet-A $\quad 5.4619 \mathrm{eV} \quad 227.00 \mathrm{~nm} \quad \mathrm{f}=0.0044 \quad<\mathrm{S}^{* *} 2>=0.000$

$\begin{array}{cc}111->125 & -0.10430 \\ 123->132 & 0.34930 \\ 123->133 & 0.49462 \\ 123->134 & 0.14389\end{array}$

Excited State $\quad 37: \quad$ Singlet-A $\quad 5.4746 \mathrm{eV} \quad 226.47 \mathrm{~nm} \quad \mathrm{f}=0.0023 \quad<\mathrm{S}^{* *} 2>=0.000$ 


$\begin{array}{lr}118->126 & -0.11498 \\ 118->127 & 0.12760 \\ 119->130 & 0.12856 \\ 120->126 & -0.12671 \\ 121->127 & 0.17188 \\ 122->127 & -0.14652 \\ 122->128 & 0.11653 \\ 123->132 & -0.11522 \\ 124->135 & 0.19165 \\ 124->138 & 0.33785 \\ 124->139 & -0.25383 \\ 124->142 & 0.13011\end{array}$

$\begin{array}{cc}\text { Excited State } 38: & \text { Singlet-A } \\ 111->125 & 0.15199 \\ 112->125 & 0.51423 \\ 119->126 & -0.14026 \\ 120->129 & 0.10784 \\ 121->128 & 0.11878 \\ 122->128 & 0.10376 \\ 123->131 & 0.24086\end{array}$

Excited State $39: \quad$ Singlet-A $\quad 5.5011 \mathrm{eV} \quad 225.38 \mathrm{~nm} \quad \mathrm{f}=0.0127 \quad<\mathrm{S} * * 2>=0.000$

$\begin{array}{lr}112->125 & 0.21383 \\ 122->127 & 0.31052 \\ 122->128 & -0.16884 \\ 124->135 & -0.10435 \\ 124->136 & 0.13395 \\ 124->138 & 0.28716 \\ 124->139 & -0.25821 \\ 124->140 & -0.13521\end{array}$

Excited State $\quad 40: \quad$ Singlet-A $\quad 5.5206 \mathrm{eV} \quad 224.58 \mathrm{~nm} \quad \mathrm{f}=0.0056 \quad<\mathrm{S}^{* *} 2>=0.000$

$\begin{array}{cc}111->125 & 0.17420 \\ 123->132 & -0.30100 \\ 123->133 & 0.39542 \\ 123->134 & -0.35885 \\ 123->135 & 0.12994\end{array}$



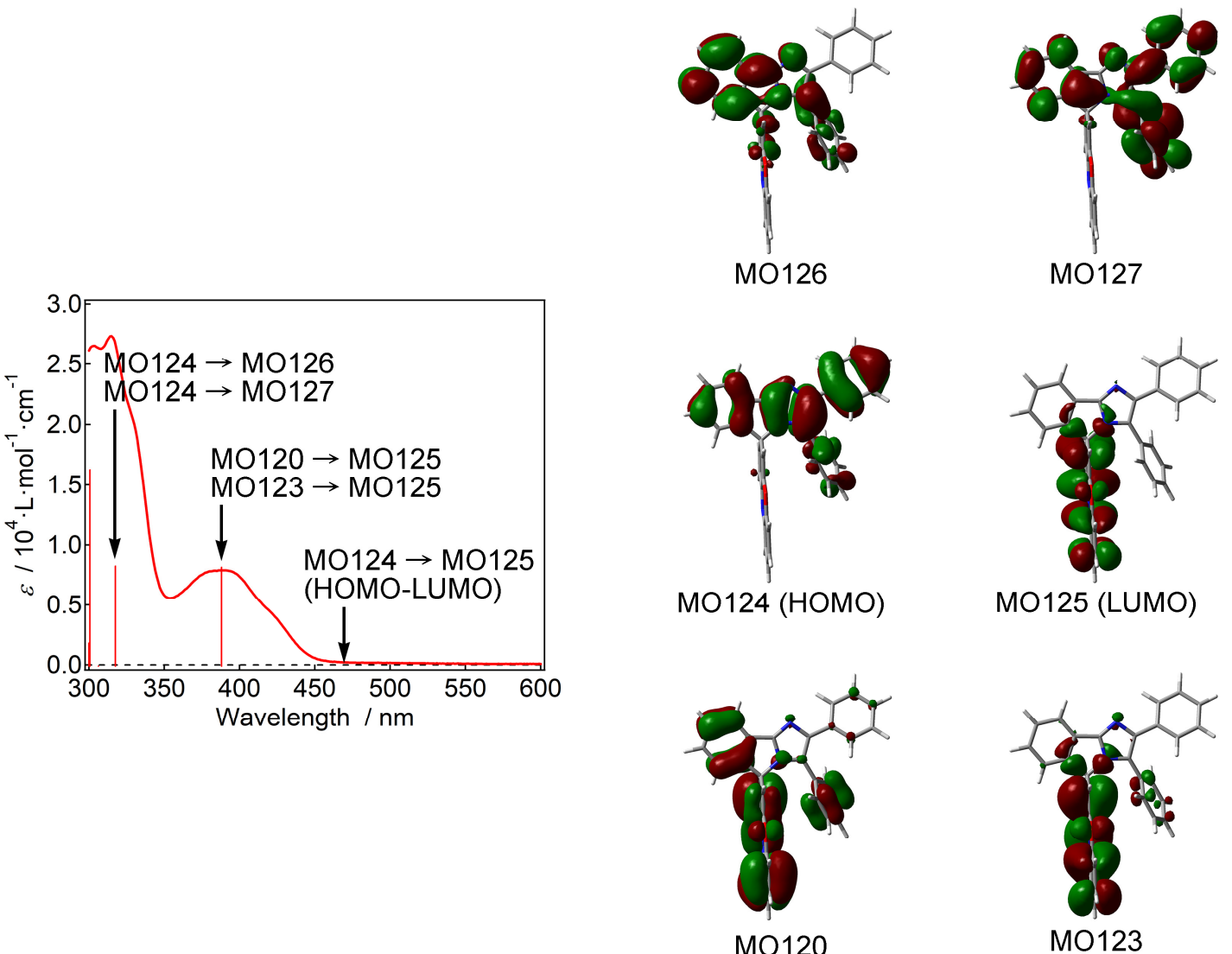

Figure S51. (a) UV-vis absorption spectrum of 2 in benzene at room temperature. The calculated spectrum (MPW1PW91/6-31G+(d)//M05-2X/6-31G(d) level of the theory) is shown by the blue vertical lines. (b) The relevant molecular orbitals of the 2 calculated at the M05-2X/6-31G(d) level of the theory.

Table S9. Standard orientation of the optimized geometry for the singlet biradical form of the ring-opening form of 2.

\begin{tabular}{ccccc}
\hline Tag & Symbol & \multicolumn{3}{c}{ Coordinates (Angstroms) } \\
\hline 1 & $\mathrm{C}$ & -1.8796900 & 5.2959650 & $\mathrm{Z}$ \\
\hline 2 & $\mathrm{C}$ & 0.1239820 & 3.3792020 & -0.2329190 \\
3 & $\mathrm{C}$ & -0.5840790 & 5.7003400 & 0.2580570 \\
4 & $\mathrm{C}$ & -2.1818120 & 3.9476870 & -0.0774730 \\
5 & $\mathrm{C}$ & -1.2071750 & 2.9709790 & -0.0003330 \\
6 & $\mathrm{C}$ & 0.3981430 & 4.7482770 & 0.3121820 \\
7 & $\mathrm{C}$ & 1.2703980 & 2.4495050 & 0.4057240 \\
8 & $\mathrm{C}$ & 3.5548730 & 0.7951750 & 0.6449740 \\
9 & $\mathrm{C}$ & 1.5711500 & 1.5220280 & -0.5918290 \\
10 & $\mathrm{C}$ & 2.1292210 & 2.5567470 & 1.5153350 \\
11 & $\mathrm{C}$ & 3.2349200 & 1.7458640 & 1.6379620 \\
12 & $\mathrm{C}$ & 2.6861000 & 0.7167090 & -0.4706110
\end{tabular}




\begin{tabular}{|c|c|c|c|c|}
\hline 13 & $\mathrm{O}$ & 2.9421590 & -0.1700560 & -1.4786770 \\
\hline 14 & $\mathrm{C}$ & 4.0451970 & -0.9650150 & -1.3644050 \\
\hline 15 & $\mathrm{C}$ & 4.8859060 & -0.8640890 & -0.2336950 \\
\hline 16 & $\mathrm{~N}$ & 4.6547740 & 0.0136390 & 0.7838460 \\
\hline 17 & $\mathrm{C}$ & 6.0069510 & -1.7174570 & -0.1794730 \\
\hline 18 & $\mathrm{C}$ & 6.2668440 & -2.6141840 & -1.1952900 \\
\hline 19 & $\mathrm{C}$ & 5.4135870 & -2.6889780 & -2.3030020 \\
\hline 20 & $\mathrm{C}$ & 4.3012790 & -1.8638560 & -2.3885030 \\
\hline 21 & $\mathrm{C}$ & -1.6628440 & 1.5931390 & 0.0761550 \\
\hline 22 & $\mathrm{~N}$ & -2.8661960 & 1.2151250 & -0.4526390 \\
\hline 23 & $\mathrm{C}$ & -2.9647160 & -0.0716810 & -0.1726250 \\
\hline 24 & $\mathrm{C}$ & -1.7730970 & -0.4597030 & 0.6153920 \\
\hline 25 & $\mathrm{~N}$ & -0.9912060 & 0.6016500 & 0.7206090 \\
\hline 26 & $\mathrm{C}$ & -4.0838420 & -0.8690560 & -0.6749430 \\
\hline 27 & $\mathrm{C}$ & -6.2424750 & -2.3011390 & -1.7311350 \\
\hline 28 & $\mathrm{C}$ & -3.9435740 & -2.2204220 & -1.0144010 \\
\hline 29 & $\mathrm{C}$ & -5.3183060 & -0.2418280 & -0.8923940 \\
\hline 30 & $\mathrm{C}$ & -6.3892040 & -0.9545760 & -1.4073690 \\
\hline 31 & $\mathrm{C}$ & -5.0162260 & -2.9282940 & -1.5400180 \\
\hline 32 & $\mathrm{C}$ & -1.4255370 & -1.7129800 & 1.2825770 \\
\hline 33 & $\mathrm{C}$ & -0.6710360 & -4.0393770 & 2.6402430 \\
\hline 34 & $\mathrm{C}$ & -0.0719890 & -1.9943140 & 1.5190420 \\
\hline 35 & $\mathrm{C}$ & -2.3947610 & -2.6062090 & 1.7570130 \\
\hline 36 & $\mathrm{C}$ & -2.0171640 & -3.7593680 & 2.4315830 \\
\hline 37 & $\mathrm{C}$ & 0.3001500 & -3.1514450 & 2.1838650 \\
\hline 38 & $\mathrm{H}$ & -2.6515430 & 6.0315140 & -0.4353040 \\
\hline 39 & $\mathrm{H}$ & -0.3327010 & 6.7556720 & 0.1168380 \\
\hline 40 & $\mathrm{H}$ & -3.1873530 & 3.6082020 & -0.4881290 \\
\hline 41 & $\mathrm{H}$ & 1.4183530 & 5.0644760 & 0.5052800 \\
\hline 42 & $\mathrm{H}$ & 0.9443800 & 1.4204340 & -1.4704220 \\
\hline 43 & $\mathrm{H}$ & 1.8956020 & 3.2746540 & 2.2946590 \\
\hline 44 & $\mathrm{H}$ & 3.8947870 & 1.8081690 & 2.4962510 \\
\hline 45 & $\mathrm{H}$ & 6.6493520 & -1.6350730 & 0.6904680 \\
\hline 46 & $\mathrm{H}$ & 7.1346180 & -3.2629580 & -1.1368340 \\
\hline 47 & $\mathrm{H}$ & 5.6192090 & -3.3943960 & -3.1013650 \\
\hline 48 & $\mathrm{H}$ & 3.6240250 & -1.9010670 & -3.2346710 \\
\hline 49 & $\mathrm{H}$ & -7.0810970 & -2.8579660 & -2.1376660 \\
\hline 50 & $\mathrm{H}$ & -2.9861820 & -2.7119980 & -0.8855990 \\
\hline 51 & $\mathrm{H}$ & -5.4142390 & 0.8103070 & -0.6499180 \\
\hline
\end{tabular}




\begin{tabular}{lllll}
52 & $\mathrm{H}$ & -7.3427360 & -0.4590060 & -1.5609940 \\
53 & $\mathrm{H}$ & -4.8907480 & -3.9728950 & -1.8070770 \\
54 & $\mathrm{H}$ & -0.3783930 & -4.9441610 & 3.1640870 \\
55 & $\mathrm{H}$ & 0.6724350 & -1.2865350 & 1.1722440 \\
56 & $\mathrm{H}$ & -3.4466640 & -2.3860600 & 1.6174430 \\
57 & $\mathrm{H}$ & -2.7789230 & -4.4382310 & 2.8019820 \\
58 & $\mathrm{H}$ & 1.3518940 & -3.3614060 & 2.3511130 \\
\hline
\end{tabular}

SCF Done: $\quad$ E(UCAM-B3LYP)

$=-1508.77868390 \quad$ A.U.

Zero-point correction

$=0.455630($ Hartree/Particle $)$

Thermal correction to Energy

$=0.482742$

Thermal correction to Enthalpy

$=0.483686$

Thermal correction to Gibbs Free Energy

$=0.394738$

Sum of electronic and zero-point Energies

$=-1508.328420$

Sum of electronic and thermal Energies

$=-1508.301308$

Sum of electronic and thermal Enthalpies

$=-1508.300363$

Sum of electronic and thermal Free Energies

$=-1508.389312$

Low frequencies --- $\begin{array}{llllll}-0.7732 & -0.5825 & -0.0007 & -0.0005 & 0.0005 & 0.7290\end{array}$

Low frequencies --- $\quad 9.6432 \quad 16.1978 \quad 23.4902$

The Results of the TDDFT calculation

Excited State $\quad 1: \quad 3.000-\mathrm{A} \quad-0.8296 \mathrm{eV} \quad-1494.54 \mathrm{~nm} \quad \mathrm{f}=-0.0000 \quad<\mathrm{S} * * 2>=2.000$

$$
\begin{array}{lr}
124 \mathrm{~A}->125 \mathrm{~A} & -0.77277 \\
124 \mathrm{~B}->125 \mathrm{~B} & 0.77277 \\
124 \mathrm{~A}<-125 \mathrm{~A} & 0.32474 \\
124 \mathrm{~B}<-125 \mathrm{~B} & -0.32474
\end{array}
$$

\begin{tabular}{|c|c|c|}
\hline Excited State 2 : & $1.000-\mathrm{A}$ & $0.8356 \mathrm{eV} 1483.86 \mathrm{~nm} \quad \mathrm{f}=0.2399$ \\
\hline $124 \mathrm{~A}->125 \mathrm{~A}$ & 0.77590 & \\
\hline $124 \mathrm{~B}->125 \mathrm{~B}$ & 0.77590 & \\
\hline $124 \mathrm{~A}<-125 \mathrm{~A}$ & -0.32886 & \\
\hline $124 \mathrm{~B}<-125 \mathrm{~B}$ & -0.32886 & \\
\hline
\end{tabular}

This state for optimization and/or second-order correction.

Total Energy, E(TD-HF/TD-DFT) $=-1508.82957633$

Copying the excited state density for this state as the 1-particle RhoCI density. 


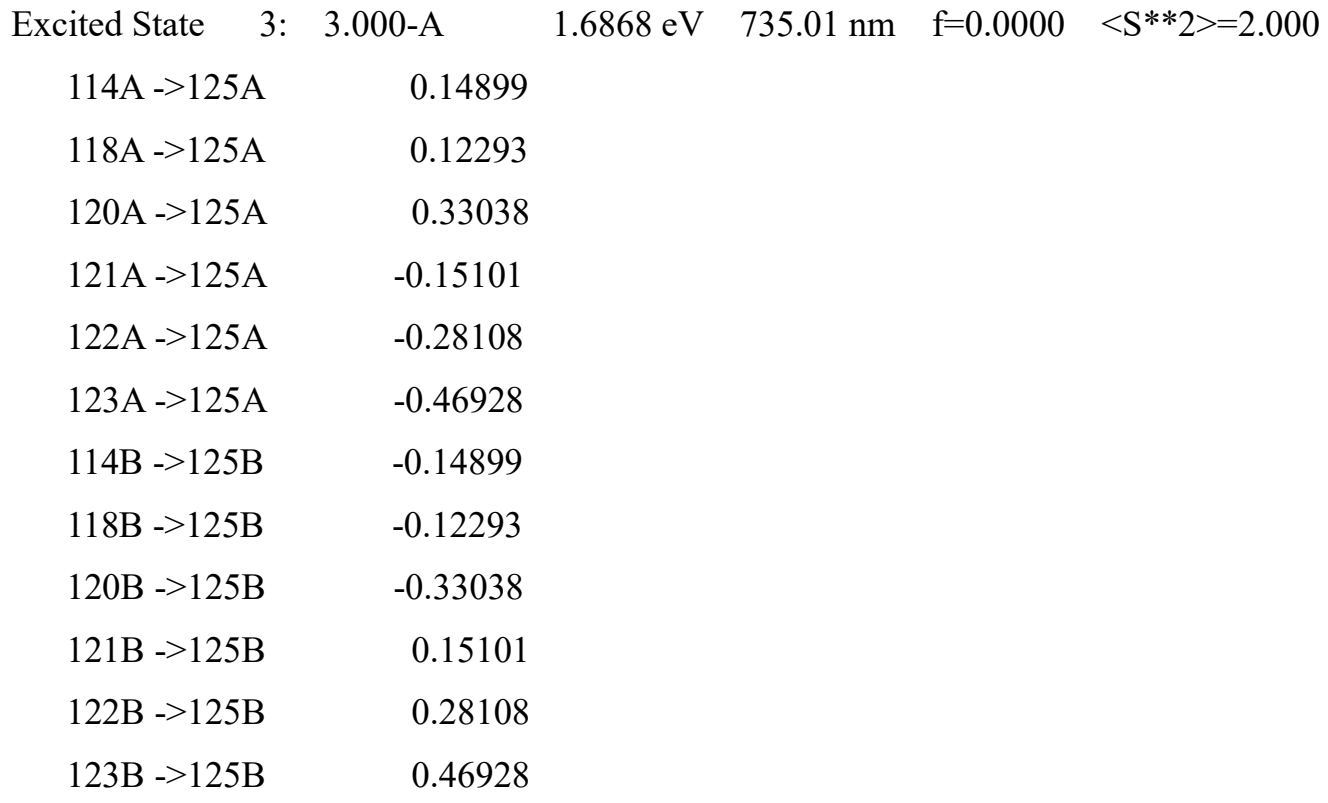

Excited State $\quad 4: \quad 3.000-\mathrm{A} \quad 2.0524 \mathrm{eV} \quad 604.10 \mathrm{~nm} \quad \mathrm{f}=0.0000 \quad<\mathrm{S} * * 2>=2.000$ $113 \mathrm{~A}->125 \mathrm{~A} \quad-0.10332$ $116 \mathrm{~A} \rightarrow 125 \mathrm{~A} \quad-0.21116$ $117 \mathrm{~A}->125 \mathrm{~A} \quad 0.28965$

$118 \mathrm{~A}->125 \mathrm{~A} \quad 0.17192$

$120 \mathrm{~A} \rightarrow 125 \mathrm{~A} \quad-0.25699$

$121 \mathrm{~A}->125 \mathrm{~A} \quad 0.15099$

$122 \mathrm{~A} \rightarrow 125 \mathrm{~A} \quad 0.27645$

$123 \mathrm{~A} \rightarrow 125 \mathrm{~A} \quad-0.31740$

$124 \mathrm{~A}->126 \mathrm{~A} \quad 0.13012$

$113 \mathrm{~B}->125 \mathrm{~B} \quad 0.10332$

$116 \mathrm{~B}->125 \mathrm{~B} \quad 0.21116$

$117 \mathrm{~B}->125 \mathrm{~B} \quad-0.28965$

$118 \mathrm{~B}->125 \mathrm{~B} \quad-0.17192$

$120 \mathrm{~B}->125 \mathrm{~B} \quad 0.25699$

$121 \mathrm{~B}->125 \mathrm{~B} \quad-0.15099$

$122 \mathrm{~B}->125 \mathrm{~B} \quad-0.27645$

$123 \mathrm{~B}->125 \mathrm{~B} \quad 0.31740$

$124 \mathrm{~B}->126 \mathrm{~B} \quad-0.13012$

Excited State $\quad 5: \quad 3.000-\mathrm{A} \quad 2.0785 \mathrm{eV} \quad 596.50 \mathrm{~nm} \quad \mathrm{f}=0.0000 \quad<\mathrm{S} * * 2>=2.000$
$114 \mathrm{~A}->125 \mathrm{~A}$
0.10142
$117 \mathrm{~A}->125 \mathrm{~A}$
$-0.38970$
$118 \mathrm{~A}->125 \mathrm{~A}$
$-0.31598$
$120 \mathrm{~A}->125 \mathrm{~A}$
$-0.21400$ 


$$
\begin{array}{lr}
121 \mathrm{~A}->125 \mathrm{~A} & 0.17480 \\
123 \mathrm{~A}->125 \mathrm{~A} & -0.32610 \\
114 \mathrm{~B}->125 \mathrm{~B} & -0.10142 \\
117 \mathrm{~B}->125 \mathrm{~B} & 0.38970 \\
118 \mathrm{~B}->125 \mathrm{~B} & 0.31598 \\
120 \mathrm{~B}->125 \mathrm{~B} & 0.21400 \\
121 \mathrm{~B}->125 \mathrm{~B} & -0.17480 \\
123 \mathrm{~B}->125 \mathrm{~B} & 0.32610
\end{array}
$$

\begin{tabular}{cr} 
Excited State $\quad 6:$ & \multicolumn{1}{c}{$1.000-\mathrm{A}$} \\
$118 \mathrm{~A}->125 \mathrm{~A}$ & -0.11767 \\
$120 \mathrm{~A}->125 \mathrm{~A}$ & -0.17697 \\
$122 \mathrm{~A}->125 \mathrm{~A}$ & 0.14291 \\
$123 \mathrm{~A}->125 \mathrm{~A}$ & 0.63247 \\
$118 \mathrm{~B}->125 \mathrm{~B}$ & -0.11767 \\
$120 \mathrm{~B}->125 \mathrm{~B}$ & -0.17697 \\
$122 \mathrm{~B}->125 \mathrm{~B}$ & 0.14291 \\
$123 \mathrm{~B}->125 \mathrm{~B}$ & 0.63247
\end{tabular}
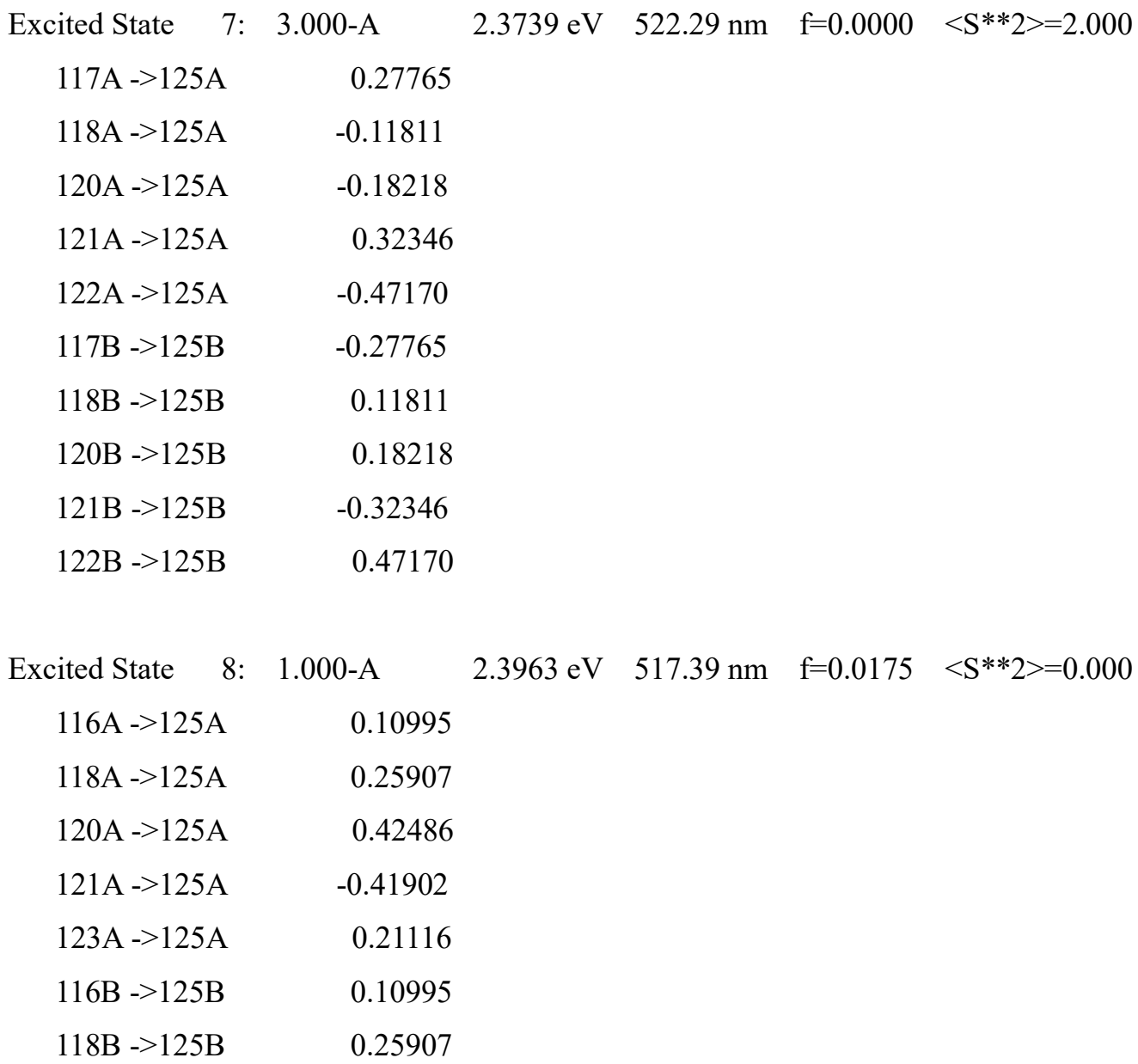


$$
\begin{array}{lr}
120 \mathrm{~B}->125 \mathrm{~B} & 0.42486 \\
121 \mathrm{~B}->125 \mathrm{~B} & -0.41902 \\
123 \mathrm{~B}->125 \mathrm{~B} & 0.21116
\end{array}
$$

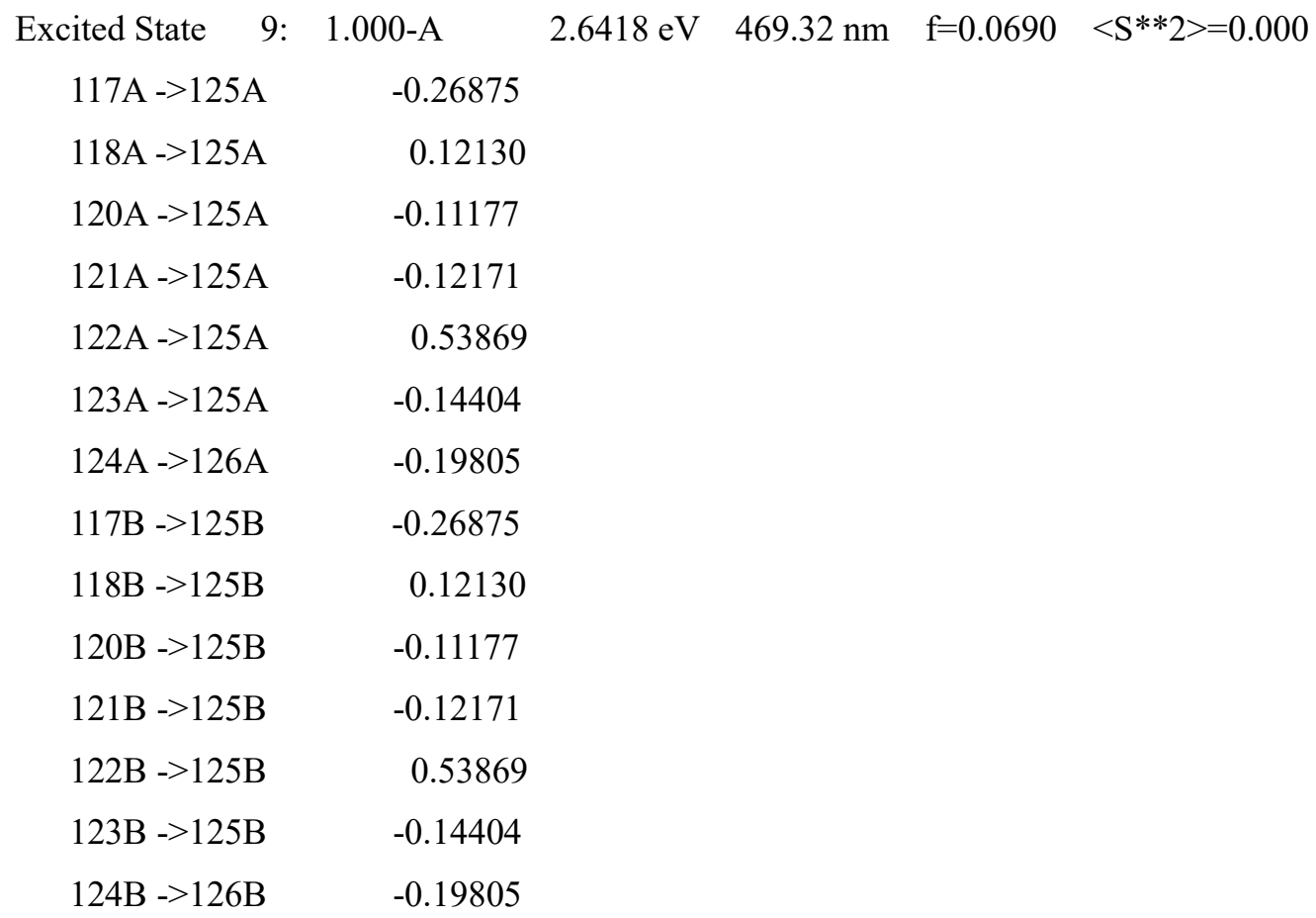

Excited State $\quad 10: \quad 3.000-\mathrm{A} \quad 2.6557 \mathrm{eV} \quad 466.87 \mathrm{~nm} \quad \mathrm{f}=0.0000 \quad<\mathrm{S} * * 2>=2.000$

$\begin{array}{lr}117 \mathrm{~A}->125 \mathrm{~A} & 0.18502 \\ 118 \mathrm{~A}->125 \mathrm{~A} & -0.39397 \\ 119 \mathrm{~A}->125 \mathrm{~A} & -0.10785 \\ 120 \mathrm{~A}->125 \mathrm{~A} & 0.20952 \\ 122 \mathrm{~A}->125 \mathrm{~A} & 0.11833 \\ 124 \mathrm{~A}->126 \mathrm{~A} & 0.28157 \\ 124 \mathrm{~A}->127 \mathrm{~A} & -0.12144 \\ 124 \mathrm{~A}->129 \mathrm{~A} & -0.23669 \\ 117 \mathrm{~B}->125 \mathrm{~B} & -0.18502 \\ 118 \mathrm{~B}->125 \mathrm{~B} & 0.39397 \\ 119 \mathrm{~B}->125 \mathrm{~B} & 0.10785 \\ 120 \mathrm{~B}->125 \mathrm{~B} & -0.20952 \\ 122 \mathrm{~B}->125 \mathrm{~B} & -0.11833 \\ 124 \mathrm{~B}->126 \mathrm{~B} & -0.28157 \\ 124 \mathrm{~B}->127 \mathrm{~B} & 0.12144 \\ 124 \mathrm{~B}->129 \mathrm{~B} & 0.23669\end{array}$

Excited State $\quad 11: \quad 3.000-\mathrm{A} \quad 2.7119 \mathrm{eV} \quad 457.18 \mathrm{~nm} \quad \mathrm{f}=0.0000 \quad<\mathrm{S} * * 2>=2.000$ 


$$
\begin{array}{lr}
114 \mathrm{~A}->125 \mathrm{~A} & 0.13605 \\
117 \mathrm{~A}->125 \mathrm{~A} & 0.24287 \\
118 \mathrm{~A}->125 \mathrm{~A} & -0.15227 \\
121 \mathrm{~A}->125 \mathrm{~A} & 0.12073 \\
122 \mathrm{~A}->125 \mathrm{~A} & 0.21086 \\
124 \mathrm{~A}->126 \mathrm{~A} & -0.52725 \\
114 \mathrm{~B}->125 \mathrm{~B} & -0.13605 \\
117 \mathrm{~B}->125 \mathrm{~B} & -0.24287 \\
118 \mathrm{~B}->125 \mathrm{~B} & 0.15227 \\
121 \mathrm{~B}->125 \mathrm{~B} & -0.12073 \\
122 \mathrm{~B}->125 \mathrm{~B} & -0.21086 \\
124 \mathrm{~B}->126 \mathrm{~B} & 0.52725
\end{array}
$$

$$
\begin{array}{cr}
\text { Excited State } \quad 12: & 3.000-\mathrm{A} \\
111 \mathrm{~A}->125 \mathrm{~A} & -0.67296 \\
111 \mathrm{~B}->125 \mathrm{~B} & 0.67296
\end{array}
$$

Excited State $\quad 13: \quad 1.000-\mathrm{A} \quad 2.8880 \mathrm{eV} \quad 429.30 \mathrm{~nm} \quad \mathrm{f}=0.0826 \quad<\mathrm{S}^{* *} 2>=0.000$

$$
\begin{array}{lr}
117 \mathrm{~A}->125 \mathrm{~A} & 0.56330 \\
118 \mathrm{~A}->125 \mathrm{~A} & 0.17219 \\
121 \mathrm{~A}>125 \mathrm{~A} & 0.11679 \\
122 \mathrm{~A}->125 \mathrm{~A} & 0.20200 \\
124 \mathrm{~A}->126 \mathrm{~A} & -0.24708 \\
117 \mathrm{~B}->125 \mathrm{~B} & 0.56330 \\
118 \mathrm{~B}->125 \mathrm{~B} & 0.17219 \\
121 \mathrm{~B}->125 \mathrm{~B} & 0.11679 \\
122 \mathrm{~B}->125 \mathrm{~B} & 0.20200 \\
124 \mathrm{~B}->126 \mathrm{~B} & -0.24708
\end{array}
$$

Excited State $\quad 14: \quad 3.000-\mathrm{A} \quad 2.9332 \mathrm{eV} \quad 422.70 \mathrm{~nm} \quad \mathrm{f}=0.0000 \quad<\mathrm{S}^{* *} 2>=2.000$

$\begin{array}{lc}116 \mathrm{~A}->125 \mathrm{~A} & -0.11224 \\ 117 \mathrm{~A}->125 \mathrm{~A} & 0.13967 \\ 118 \mathrm{~A}->125 \mathrm{~A} & -0.26201 \\ 119 \mathrm{~A}->125 \mathrm{~A} & 0.35416 \\ 120 \mathrm{~A}->125 \mathrm{~A} & -0.17101 \\ 121 \mathrm{~A}->125 \mathrm{~A} & -0.43047 \\ 116 \mathrm{~B}->125 \mathrm{~B} & 0.11224 \\ 117 \mathrm{~B}->125 \mathrm{~B} & -0.13967 \\ 118 \mathrm{~B}->125 \mathrm{~B} & 0.26201\end{array}$




$$
\begin{array}{lr}
119 \mathrm{~B}->125 \mathrm{~B} & -0.35416 \\
120 \mathrm{~B}->125 \mathrm{~B} & 0.17101 \\
121 \mathrm{~B}->125 \mathrm{~B} & 0.43047
\end{array}
$$
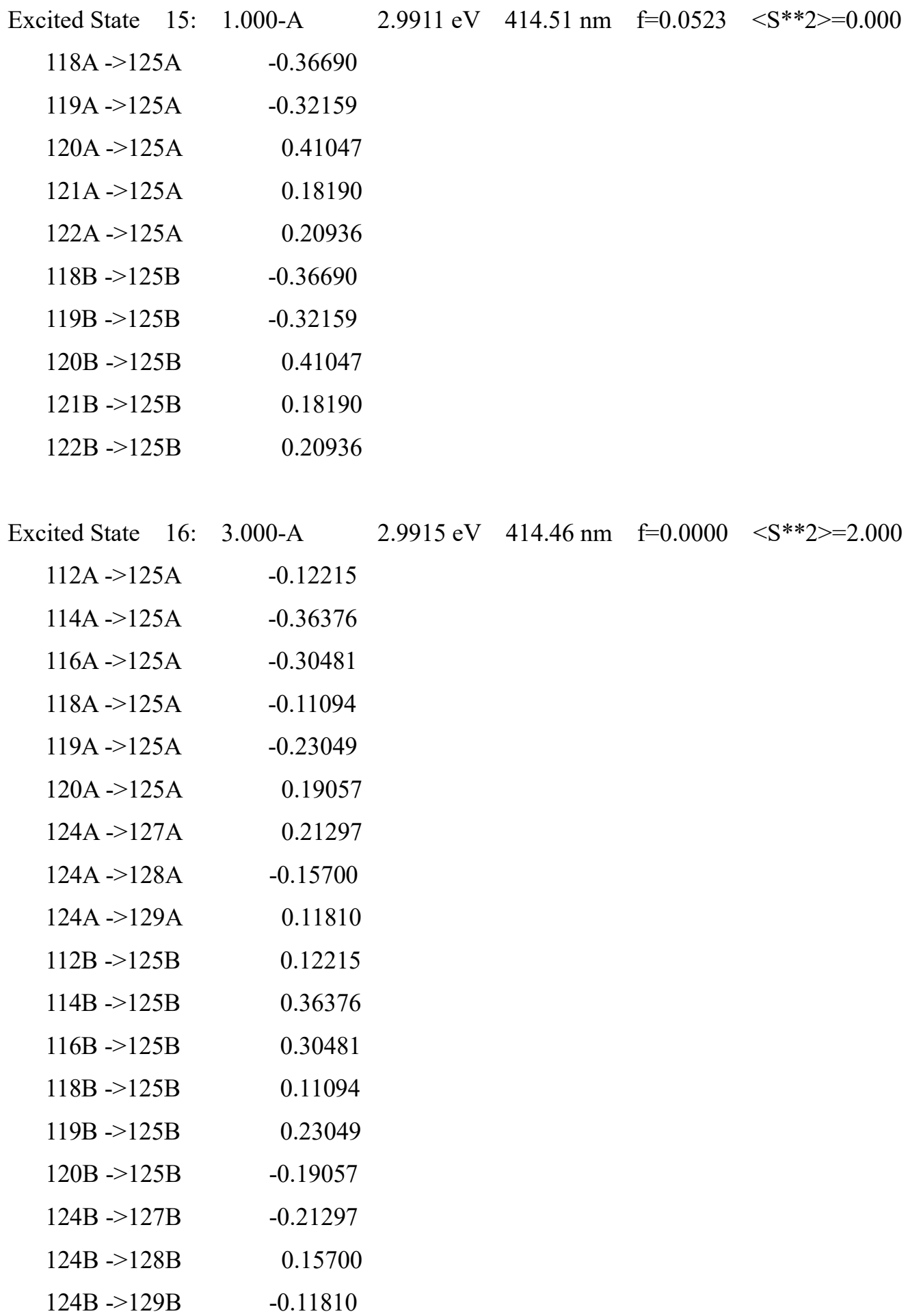

Excited State $\quad 17: \quad 3.000-\mathrm{A} \quad 3.0405 \mathrm{eV} \quad 407.77 \mathrm{~nm} \quad \mathrm{f}=0.0000 \quad<\mathrm{S} * * 2>=2.000$
$112 \mathrm{~A}->125 \mathrm{~A}$
$-0.15178$
$113 \mathrm{~A}->125 \mathrm{~A}$
0.28345 


$\begin{array}{lr}115 \mathrm{~A}->125 \mathrm{~A} & -0.10799 \\ 116 \mathrm{~A}->125 \mathrm{~A} & -0.20229 \\ 121 \mathrm{~A}>125 \mathrm{~A} & -0.11721 \\ 123 \mathrm{~A}->125 \mathrm{~A} & -0.10414 \\ 124 \mathrm{~A}->126 \mathrm{~A} & -0.13276 \\ 124 \mathrm{~A}->127 \mathrm{~A} & -0.34140 \\ 124 \mathrm{~A}->128 \mathrm{~A} & 0.22830 \\ 124 \mathrm{~A}->129 \mathrm{~A} & 0.13575 \\ 124 \mathrm{~A}->130 \mathrm{~A} & 0.14356 \\ 112 \mathrm{~B}->125 \mathrm{~B} & 0.15178 \\ 113 \mathrm{~B}->125 \mathrm{~B} & -0.28345 \\ 115 \mathrm{~B}->125 \mathrm{~B} & 0.10799 \\ 116 \mathrm{~B}->125 \mathrm{~B} & 0.20229 \\ 121 \mathrm{~B}->125 \mathrm{~B} & 0.11721 \\ 123 \mathrm{~B}->125 \mathrm{~B} & 0.10414 \\ 124 \mathrm{~B}->126 \mathrm{~B} & 0.13276 \\ 124 \mathrm{~B}->127 \mathrm{~B} & 0.34140 \\ 124 \mathrm{~B}->128 \mathrm{~B} & -0.22830 \\ 124 \mathrm{~B}->129 \mathrm{~B} & -0.13575 \\ 124 \mathrm{~B}->130 \mathrm{~B} & -0.14356\end{array}$

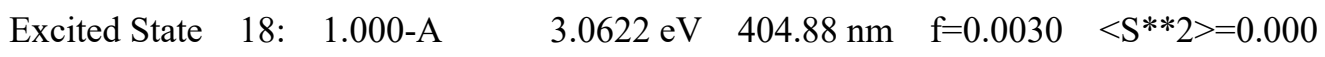

$\begin{array}{lr}116 \mathrm{~A}->125 \mathrm{~A} & 0.11226 \\ 117 \mathrm{~A}->125 \mathrm{~A} & -0.18698 \\ 118 \mathrm{~A}->125 \mathrm{~A} & 0.44746 \\ 119 \mathrm{~A}->125 \mathrm{~A} & -0.28086 \\ 121 \mathrm{~A}->125 \mathrm{~A} & 0.39653 \\ 116 \mathrm{~B}->125 \mathrm{~B} & 0.11226 \\ 117 \mathrm{~B}->125 \mathrm{~B} & -0.18698 \\ 118 \mathrm{~B}->125 \mathrm{~B} & 0.44746 \\ 119 \mathrm{~B}->125 \mathrm{~B} & -0.28086 \\ 121 \mathrm{~B}->125 \mathrm{~B} & 0.39653\end{array}$

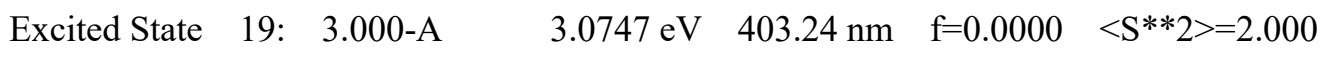
$112 \mathrm{~A}->125 \mathrm{~A}$
0.19230
$113 \mathrm{~A}->125 \mathrm{~A}$
$-0.46620$
$116 \mathrm{~A}->125 \mathrm{~A}$
$-0.19960$
$124 \mathrm{~A}->128 \mathrm{~A}$
0.14133
$124 \mathrm{~A}->129 \mathrm{~A}$
0.15225 


$$
\begin{array}{lr}
124 \mathrm{~A}->130 \mathrm{~A} & 0.21609 \\
112 \mathrm{~B}->125 \mathrm{~B} & -0.19230 \\
113 \mathrm{~B}->125 \mathrm{~B} & 0.46620 \\
116 \mathrm{~B}->125 \mathrm{~B} & 0.19960 \\
124 \mathrm{~B}->128 \mathrm{~B} & -0.14133 \\
124 \mathrm{~B}->129 \mathrm{~B} & -0.15225 \\
124 \mathrm{~B}->130 \mathrm{~B} & -0.21609
\end{array}
$$

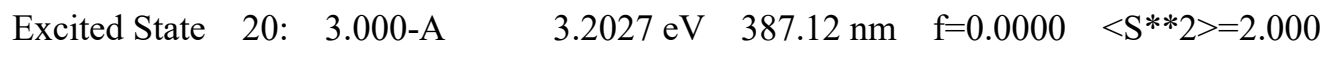

$\begin{array}{ll}112 \mathrm{~A}->125 \mathrm{~A} & 0.19467 \\ 114 \mathrm{~A}->125 \mathrm{~A} & 0.16314 \\ 116 \mathrm{~A}->125 \mathrm{~A} & -0.22155 \\ 119 \mathrm{~A}->125 \mathrm{~A} & -0.41459 \\ 120 \mathrm{~A}->125 \mathrm{~A} & -0.22315 \\ 121 \mathrm{~A}->125 \mathrm{~A} & -0.20187 \\ 124 \mathrm{~A}->129 \mathrm{~A} & -0.20223 \\ 124 \mathrm{~A}->130 \mathrm{~A} & -0.11996 \\ 112 \mathrm{~B}->125 \mathrm{~B} & -0.19467 \\ 114 \mathrm{~B}->125 \mathrm{~B} & -0.16314 \\ 116 \mathrm{~B}->125 \mathrm{~B} & 0.22155 \\ 119 \mathrm{~B}->125 \mathrm{~B} & 0.41459 \\ 120 \mathrm{~B}->125 \mathrm{~B} & 0.22315 \\ 121 \mathrm{~B}->125 \mathrm{~B} & 0.20187 \\ 124 \mathrm{~B}->129 \mathrm{~B} & 0.20223 \\ 124 \mathrm{~B}->130 \mathrm{~B} & 0.11996\end{array}$

Table S10. Standard orientation of the optimized geometry for the triplet biradical form of the ring-opening form of 2 .

\begin{tabular}{ccccc}
\hline Tag & Symbol & \multicolumn{3}{c}{ Coordinates (Angstroms) } \\
& & $\mathrm{X}$ & $\mathrm{Y}$ & $\mathrm{Z}$ \\
\hline 1 & $\mathrm{C}$ & -1.8958330 & 5.2866040 & -0.2135670 \\
2 & $\mathrm{C}$ & 0.0958460 & 3.3606420 & 0.2748320 \\
3 & $\mathrm{C}$ & -0.5948840 & 5.6837860 & 0.0759630 \\
4 & $\mathrm{C}$ & -2.2080910 & 3.9390100 & -0.2383560 \\
5 & $\mathrm{C}$ & -1.2370640 & 2.9589490 & 0.0252550 \\
6 & $\mathrm{C}$ & 0.3835010 & 4.7249540 & 0.3096710 \\
7 & $\mathrm{C}$ & 1.2353110 & 2.4181050 & 0.4437960 \\
8 & $\mathrm{C}$ & 3.4884790 & 0.7354320 & 0.7324400
\end{tabular}




\begin{tabular}{|c|c|c|c|c|}
\hline 9 & $\mathrm{C}$ & 1.6234310 & 1.5870730 & -0.6043010 \\
\hline 10 & $\mathrm{C}$ & 1.9871640 & 2.4185350 & 1.6303910 \\
\hline 11 & $\mathrm{C}$ & 3.0811960 & 1.5931780 & 1.7747290 \\
\hline 12 & $\mathrm{C}$ & 2.7247590 & 0.7642960 & -0.4578030 \\
\hline 13 & $\mathrm{O}$ & 3.0684980 & -0.0295690 & -1.5152260 \\
\hline 14 & $\mathrm{C}$ & 4.1550320 & -0.8429620 & -1.3755980 \\
\hline 15 & $\mathrm{C}$ & 4.8927160 & -0.8505610 & -0.1701190 \\
\hline 16 & $\mathrm{~N}$ & 4.5746450 & -0.0663920 & 0.8968910 \\
\hline 17 & $\mathrm{C}$ & 6.0037100 & -1.7168040 & -0.0951710 \\
\hline 18 & $\mathrm{C}$ & 6.3502400 & -2.5217300 & -1.1603240 \\
\hline 19 & $\mathrm{C}$ & 5.5976580 & -2.4898590 & -2.3411250 \\
\hline 20 & $\mathrm{C}$ & 4.4987030 & -1.6498240 & -2.4494740 \\
\hline 21 & $\mathrm{C}$ & -1.6907010 & 1.5774520 & 0.0855770 \\
\hline 22 & $\mathrm{~N}$ & -2.9075140 & 1.2099220 & -0.4191220 \\
\hline 23 & $\mathrm{C}$ & -2.9964350 & -0.0834470 & -0.1674400 \\
\hline 24 & $\mathrm{C}$ & -1.7814310 & -0.4881870 & 0.5759050 \\
\hline 25 & $\mathrm{~N}$ & -0.9978530 & 0.5731200 & 0.6826310 \\
\hline 26 & $\mathrm{C}$ & -4.1276870 & -0.8703550 & -0.6589880 \\
\hline 27 & $\mathrm{C}$ & -6.3123710 & -2.2790730 & -1.6925410 \\
\hline 28 & $\mathrm{C}$ & -3.9933740 & -2.2104930 & -1.0424930 \\
\hline 29 & $\mathrm{C}$ & -5.3698900 & -0.2417220 & -0.8211660 \\
\hline 30 & $\mathrm{C}$ & -6.4535310 & -0.9432610 & -1.3247660 \\
\hline 31 & $\mathrm{C}$ & -5.0790200 & -2.9067350 & -1.5568360 \\
\hline 32 & $\mathrm{C}$ & -1.4107730 & -1.7553280 & 1.2014850 \\
\hline 33 & $\mathrm{C}$ & -0.6086880 & -4.1111080 & 2.4790520 \\
\hline 34 & $\mathrm{C}$ & -0.0501510 & -2.0287870 & 1.4066870 \\
\hline 35 & $\mathrm{C}$ & -2.3624310 & -2.6716020 & 1.6681850 \\
\hline 36 & $\mathrm{C}$ & -1.9611460 & -3.8392860 & 2.3027660 \\
\hline 37 & $\mathrm{C}$ & 0.3453280 & -3.2002890 & 2.0313040 \\
\hline 38 & $\mathrm{H}$ & -2.6653420 & 6.0257590 & -0.4118170 \\
\hline 39 & $\mathrm{H}$ & -0.3355110 & 6.7375250 & 0.1053870 \\
\hline 40 & $\mathrm{H}$ & -3.2178040 & 3.6054100 & -0.4454860 \\
\hline 41 & $\mathrm{H}$ & 1.4069990 & 5.0335110 & 0.4983100 \\
\hline 42 & $\mathrm{H}$ & 1.0748200 & 1.5689950 & -1.5393770 \\
\hline 43 & $\mathrm{H}$ & 1.6845070 & 3.0663730 & 2.4464820 \\
\hline 44 & $\mathrm{H}$ & 3.6637560 & 1.5746130 & 2.6892400 \\
\hline 45 & $\mathrm{H}$ & 6.5671680 & -1.7183990 & 0.8315280 \\
\hline 46 & $\mathrm{H}$ & 7.2084400 & -3.1813860 & -1.0844640 \\
\hline 47 & $\mathrm{H}$ & 5.8717520 & -3.1234560 & -3.1781860 \\
\hline
\end{tabular}




\begin{tabular}{lllll}
48 & $\mathrm{H}$ & 3.8985700 & -1.6050690 & -3.3516520 \\
49 & $\mathrm{H}$ & -7.1610400 & -2.8269520 & -2.0902770 \\
50 & $\mathrm{H}$ & -3.0308980 & -2.7016650 & -0.9572440 \\
51 & $\mathrm{H}$ & -5.4617030 & 0.8024600 & -0.5450440 \\
52 & $\mathrm{H}$ & -7.4127580 & -0.4471740 & -1.4351600 \\
53 & $\mathrm{H}$ & -4.9582940 & -3.9423900 & -1.8586430 \\
54 & $\mathrm{H}$ & -0.2976560 & -5.0271640 & 2.9717890 \\
55 & $\mathrm{H}$ & 0.6807340 & -1.3027800 & 1.0689980 \\
56 & $\mathrm{H}$ & -3.4188140 & -2.4584040 & 1.5547060 \\
57 & $\mathrm{H}$ & -2.7092470 & -4.5361080 & 2.6675940 \\
58 & $\mathrm{H}$ & 1.4019550 & -3.4033120 & 2.1745150 \\
\hline
\end{tabular}

SCF Done: $\quad$ E(UCAM-B3LYP)

$=-1508.77467716 \quad$ A.U.

Zero-point correction

$=0.455607$ (Hartree/Particle)

Thermal correction to Energy

$=0.482757$

Thermal correction to Enthalpy

$=0.483701$

Thermal correction to Gibbs Free Energy

$=0.393319$

Sum of electronic and zero-point Energies

$=-1508.327644$

Sum of electronic and thermal Energies

$=-1508.300494$

Sum of electronic and thermal Enthalpies

$=-1508.299550$

Sum of electronic and thermal Free Energies

$=-1508.389931$

$\begin{array}{lllllll}\text { Low frequencies --- } & -1.5469 & -0.9513 & -0.0006 & -0.0006 & -0.0001 & 0.9380\end{array}$

Low frequencies --- $\quad 8.8097 \quad 15.3052 \quad 20.2123$

The Results of the TDDFT calculation

Excited State $\quad 1: \quad 3.121-\mathrm{A} \quad 2.0848 \mathrm{eV} \quad 594.72 \mathrm{~nm} \quad \mathrm{f}=0.0134 \quad<\mathrm{S} * * 2>=2.185$

$$
\begin{array}{lr}
124 \mathrm{~A}->126 \mathrm{~A} & 0.13441 \\
110 \mathrm{~B}->124 \mathrm{~B} & -0.11064 \\
111 \mathrm{~B}->124 \mathrm{~B} & -0.20698 \\
112 \mathrm{~B}->124 \mathrm{~B} & 0.28658 \\
121 \mathrm{~B}->124 \mathrm{~B} & 0.75459 \\
122 \mathrm{~B}->124 \mathrm{~B} & -0.24276 \\
123 \mathrm{~B}->124 \mathrm{~B} & 0.36817
\end{array}
$$

This state for optimization and/or second-order correction.

Total Energy, E(TD-HF/TD-DFT) $=-1508.75551643$

Copying the excited state density for this state as the 1-particle RhoCI density. 


$\begin{array}{cr}\text { Excited State } \quad 2: & 3.085-\mathrm{A} \\ 113 \mathrm{~B}->124 \mathrm{~B} & -0.26308 \\ 114 \mathrm{~B}->124 \mathrm{~B} & 0.66275 \\ 116 \mathrm{~B}->124 \mathrm{~B} & 0.15918 \\ 118 \mathrm{~B}->124 \mathrm{~B} & 0.12705 \\ 119 \mathrm{~B}->124 \mathrm{~B} & 0.22165 \\ 120 \mathrm{~B}->124 \mathrm{~B} & 0.46153 \\ 121 \mathrm{~B}->124 \mathrm{~B} & 0.21812 \\ 122 \mathrm{~B}->124 \mathrm{~B} & 0.13112 \\ 123 \mathrm{~B}->124 \mathrm{~B} & -0.24047\end{array}$

$\begin{array}{cr}\text { Excited State } 3: & 3.259-\mathrm{A} \\ 122 \mathrm{~A}->126 \mathrm{~A} & -0.10614 \\ 124 \mathrm{~A}->127 \mathrm{~A} & -0.20640 \\ 125 \mathrm{~A}->127 \mathrm{~A} & -0.13149 \\ 125 \mathrm{~A}->128 \mathrm{~A} & 0.10661 \\ 112 \mathrm{~B}->124 \mathrm{~B} & -0.12055 \\ 113 \mathrm{~B}->127 \mathrm{~B} & -0.10893 \\ 114 \mathrm{~B}->124 \mathrm{~B} & -0.16686 \\ 120 \mathrm{~B}->124 \mathrm{~B} & 0.59709 \\ 121 \mathrm{~B}->124 \mathrm{~B} & -0.21676 \\ 121 \mathrm{~B}->126 \mathrm{~B} & 0.10692 \\ 122 \mathrm{~B}->124 \mathrm{~B} & -0.33666 \\ 122 \mathrm{~B}->125 \mathrm{~B} & 0.11837 \\ 123 \mathrm{~B}->124 \mathrm{~B} & 0.33507 \\ 123 \mathrm{~B}->125 \mathrm{~B} & -0.26269\end{array}$

Excited State $\quad 4: \quad 3.207-\mathrm{A} \quad 2.8506 \mathrm{eV} \quad 434.94 \mathrm{~nm} \quad \mathrm{f}=0.0448 \quad<\mathrm{S}^{* *} 2>=2.321$

$\begin{array}{lr}124 \mathrm{~A}->127 \mathrm{~A} & -0.11275 \\ 125 \mathrm{~A}->127 \mathrm{~A} & 0.12138 \\ 125 \mathrm{~A}->128 \mathrm{~A} & -0.25736 \\ 125 \mathrm{~A}->137 \mathrm{~A} & 0.12167 \\ 114 \mathrm{~B}->124 \mathrm{~B} & -0.21332 \\ 118 \mathrm{~B}->125 \mathrm{~B} & -0.11277 \\ 120 \mathrm{~B}->124 \mathrm{~B} & 0.29636 \\ 121 \mathrm{~B}->125 \mathrm{~B} & -0.12405 \\ 122 \mathrm{~B}->124 \mathrm{~B} & -0.12777 \\ 123 \mathrm{~B}->124 \mathrm{~B} & -0.11066\end{array}$


$123 \mathrm{~B}->125 \mathrm{~B} \quad 0.74165$

Excited State $\quad 5: \quad 3.080-\mathrm{A} \quad 2.8772 \mathrm{eV} \quad 430.92 \mathrm{~nm} \quad \mathrm{f}=0.0013 \quad<\mathrm{S} * * 2>=2.122$
$115 \mathrm{~B}->124 \mathrm{~B}$
$-0.15648$
$115 \mathrm{~B}->125 \mathrm{~B}$
0.93288
$115 \mathrm{~B}->136 \mathrm{~B}$
0.11043
$115 \mathrm{~B}->157 \mathrm{~B}$
$-0.12974$

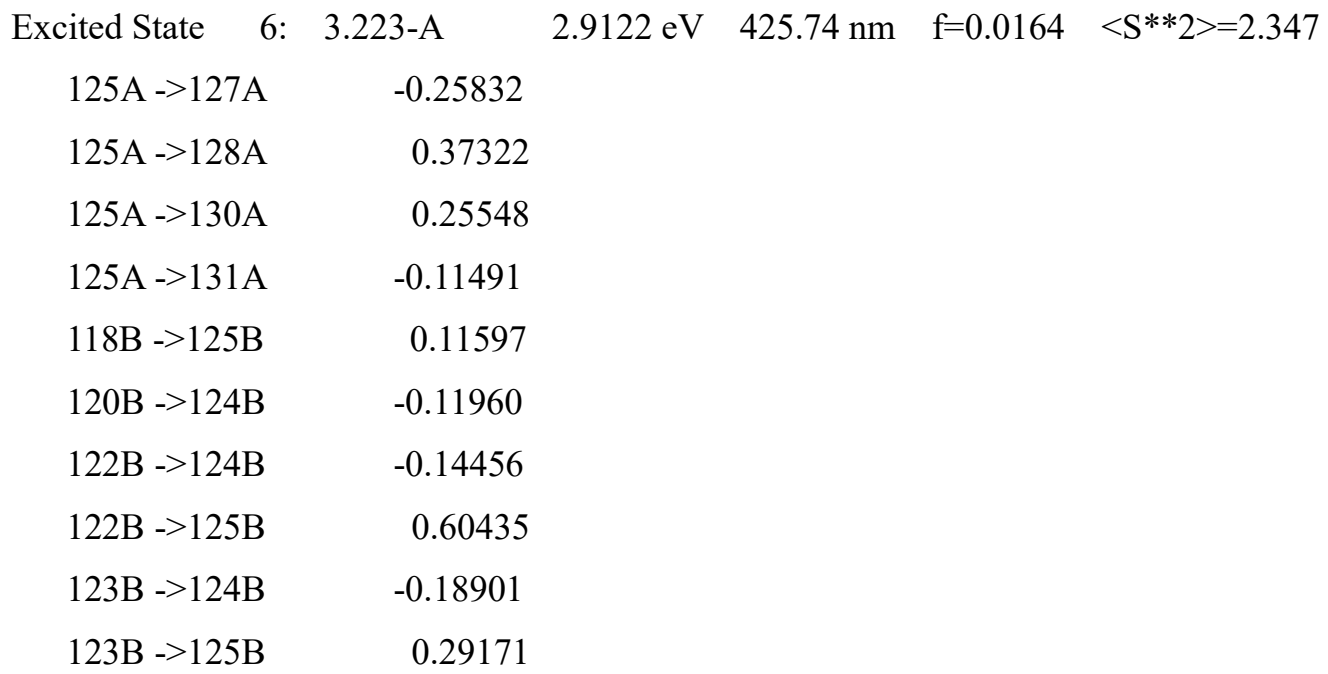

Excited State $\quad 7: \quad 3.131-\mathrm{A} \quad 3.0944 \mathrm{eV} \quad 400.68 \mathrm{~nm} \quad \mathrm{f}=0.0059 \quad<\mathrm{S}^{* *} 2>=2.200$
$110 \mathrm{~B}->124 \mathrm{~B}$
0.17117
111B ->124B
0.22894
$112 \mathrm{~B}->124 \mathrm{~B}$
0.69746
114B ->124B
$-0.26245$
117B ->124B
$-0.18784$
120B - $>124 \mathrm{~B}$
0.16628
$122 \mathrm{~B}->124 \mathrm{~B}$
0.16081
123B ->124B
$-0.34356$
$123 \mathrm{~B}->125 \mathrm{~B}$
$-0.18227$

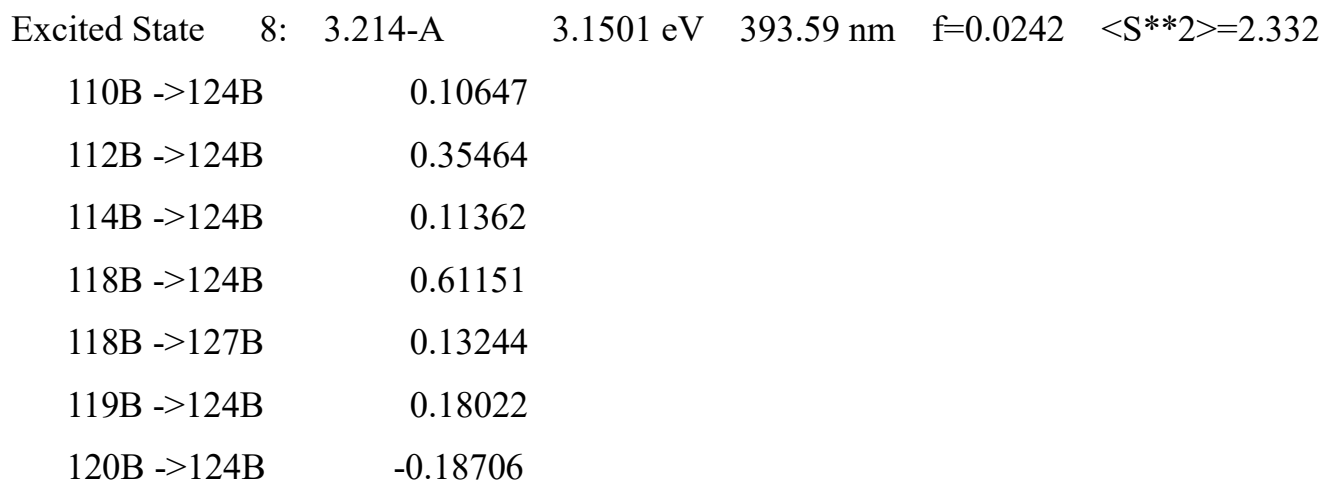




$$
\begin{array}{lr}
121 \mathrm{~B}->124 \mathrm{~B} & -0.24516 \\
122 \mathrm{~B}->124 \mathrm{~B} & -0.18911 \\
123 \mathrm{~B}->124 \mathrm{~B} & 0.32194 \\
123 \mathrm{~B}->125 \mathrm{~B} & 0.14922
\end{array}
$$

Excited State $\quad 9: \quad 3.606-\mathrm{A} \quad 3.2954 \mathrm{eV} \quad 376.24 \mathrm{~nm} \quad \mathrm{f}=0.0198 \quad<\mathrm{S} * * 2>=3.002$

$$
\begin{array}{lr}
118 \mathrm{~A}->129 \mathrm{~A} & 0.10153 \\
120 \mathrm{~A}->127 \mathrm{~A} & -0.19666 \\
121 \mathrm{~A}->127 \mathrm{~A} & 0.11441 \\
122 \mathrm{~A}->126 \mathrm{~A} & -0.17736 \\
124 \mathrm{~A}->127 \mathrm{~A} & 0.22013 \\
124 \mathrm{~A}->136 \mathrm{~A} & -0.14309 \\
124 \mathrm{~A}->138 \mathrm{~A} & -0.12619 \\
112 \mathrm{~B}->124 \mathrm{~B} & 0.16468 \\
113 \mathrm{~B}->124 \mathrm{~B} & 0.36448 \\
114 \mathrm{~B}->124 \mathrm{~B} & 0.24293 \\
116 \mathrm{~B}->124 \mathrm{~B} & -0.13426 \\
119 \mathrm{~B}->124 \mathrm{~B} & -0.25262 \\
120 \mathrm{~B}->124 \mathrm{~B} & 0.11355 \\
120 \mathrm{~B}->127 \mathrm{~B} & -0.19819 \\
121 \mathrm{~B}->126 \mathrm{~B} & 0.17356 \\
122 \mathrm{~B}->124 \mathrm{~B} & 0.20678 \\
122 \mathrm{~B}->125 \mathrm{~B} & 0.10876 \\
122 \mathrm{~B}->127 \mathrm{~B} & 0.10720 \\
123 \mathrm{~B}->124 \mathrm{~B} & 0.24048
\end{array}
$$

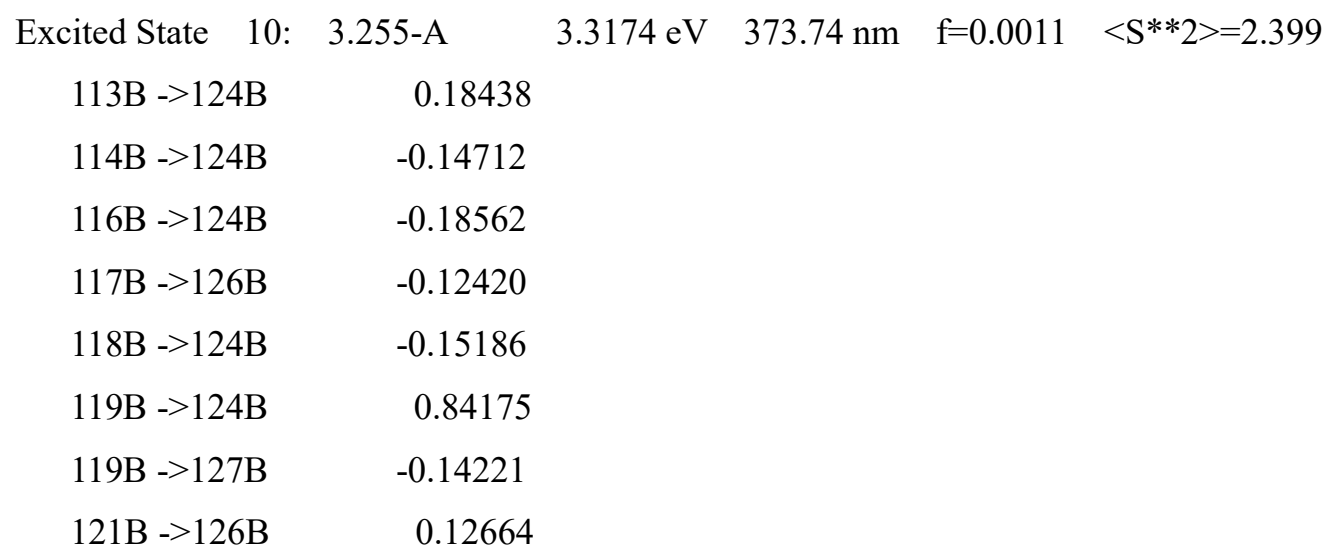

Excited State $\quad 11: \quad 3.453-\mathrm{A} \quad 3.3860 \mathrm{eV} \quad 366.17 \mathrm{~nm} \quad \mathrm{f}=0.0245 \quad<\mathrm{S} * * 2>=2.731$
$119 \mathrm{~A}->129 \mathrm{~A}$
$-0.10880$
$120 \mathrm{~A}->126 \mathrm{~A}$
$-0.11260$ 


$\begin{array}{lr}120 \mathrm{~A}->127 \mathrm{~A} & 0.12572 \\ 124 \mathrm{~A}->126 \mathrm{~A} & -0.33799 \\ 111 \mathrm{~B}->124 \mathrm{~B} & -0.23339 \\ 112 \mathrm{~B}->124 \mathrm{~B} & 0.10892 \\ 113 \mathrm{~B}->124 \mathrm{~B} & -0.23974 \\ 116 \mathrm{~B}->124 \mathrm{~B} & -0.17744 \\ 117 \mathrm{~B}->124 \mathrm{~B} & 0.19476 \\ 118 \mathrm{~B}->124 \mathrm{~B} & -0.23933 \\ 120 \mathrm{~B}->126 \mathrm{~B} & -0.10580 \\ 120 \mathrm{~B}->127 \mathrm{~B} & 0.14548 \\ 122 \mathrm{~B}->124 \mathrm{~B} & 0.38166 \\ 123 \mathrm{~B}->124 \mathrm{~B} & 0.30981 \\ 123 \mathrm{~B}->125 \mathrm{~B} & 0.14373\end{array}$

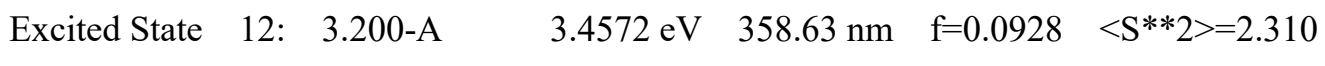

$124 \mathrm{~A} \rightarrow 126 \mathrm{~A} \quad-0.17396$

$124 \mathrm{~A} \rightarrow 127 \mathrm{~A} \quad 0.10917$

$125 \mathrm{~A} \rightarrow 127 \mathrm{~A} \quad 0.31310$

$125 \mathrm{~A} \rightarrow 128 \mathrm{~A} \quad-0.31980$

$125 \mathrm{~A}->130 \mathrm{~A} \quad-0.39971$

$125 \mathrm{~A}->131 \mathrm{~A} \quad-0.13531$

$111 \mathrm{~B}->124 \mathrm{~B} \quad-0.10129$

$116 \mathrm{~B}->125 \mathrm{~B} \quad 0.27848$

$118 \mathrm{~B}->124 \mathrm{~B} \quad-0.12747$

122B $->124 \mathrm{~B} \quad-0.19651$

$122 \mathrm{~B}->125 \mathrm{~B} \quad 0.42501$

$123 \mathrm{~B}->124 \mathrm{~B} \quad-0.15699$

123B $->125 \mathrm{~B} \quad-0.19983$

Excited State $\quad 13: \quad 3.375-\mathrm{A} \quad 3.4730 \mathrm{eV} \quad 356.99 \mathrm{~nm} \quad \mathrm{f}=0.0354 \quad<\mathrm{S}^{* *} 2>=2.598$
$118 \mathrm{~A}->133 \mathrm{~A}$
0.10429
$119 \mathrm{~A}->129 \mathrm{~A}$
0.11414
$120 \mathrm{~A} \rightarrow 126 \mathrm{~A}$
0.14374
$124 \mathrm{~A} \rightarrow 126 \mathrm{~A}$
0.31890
$125 \mathrm{~A} \rightarrow 126 \mathrm{~A}$
0.12092
$125 \mathrm{~A}->130 \mathrm{~A}$
$-0.15760$
$110 \mathrm{~B}->124 \mathrm{~B}$
0.15443
$111 \mathrm{~B}->124 \mathrm{~B}$
0.21087
$113 \mathrm{~B}->124 \mathrm{~B}$
$-0.11017$ 


$$
\begin{array}{lr}
116 \mathrm{~B}->124 \mathrm{~B} & -0.27226 \\
116 \mathrm{~B}->125 \mathrm{~B} & 0.18105 \\
117 \mathrm{~B}->124 \mathrm{~B} & -0.20640 \\
120 \mathrm{~B}->126 \mathrm{~B} & 0.12841 \\
122 \mathrm{~B}->124 \mathrm{~B} & 0.40169 \\
122 \mathrm{~B}->125 \mathrm{~B} & 0.20391 \\
123 \mathrm{~B}->124 \mathrm{~B} & 0.35054
\end{array}
$$
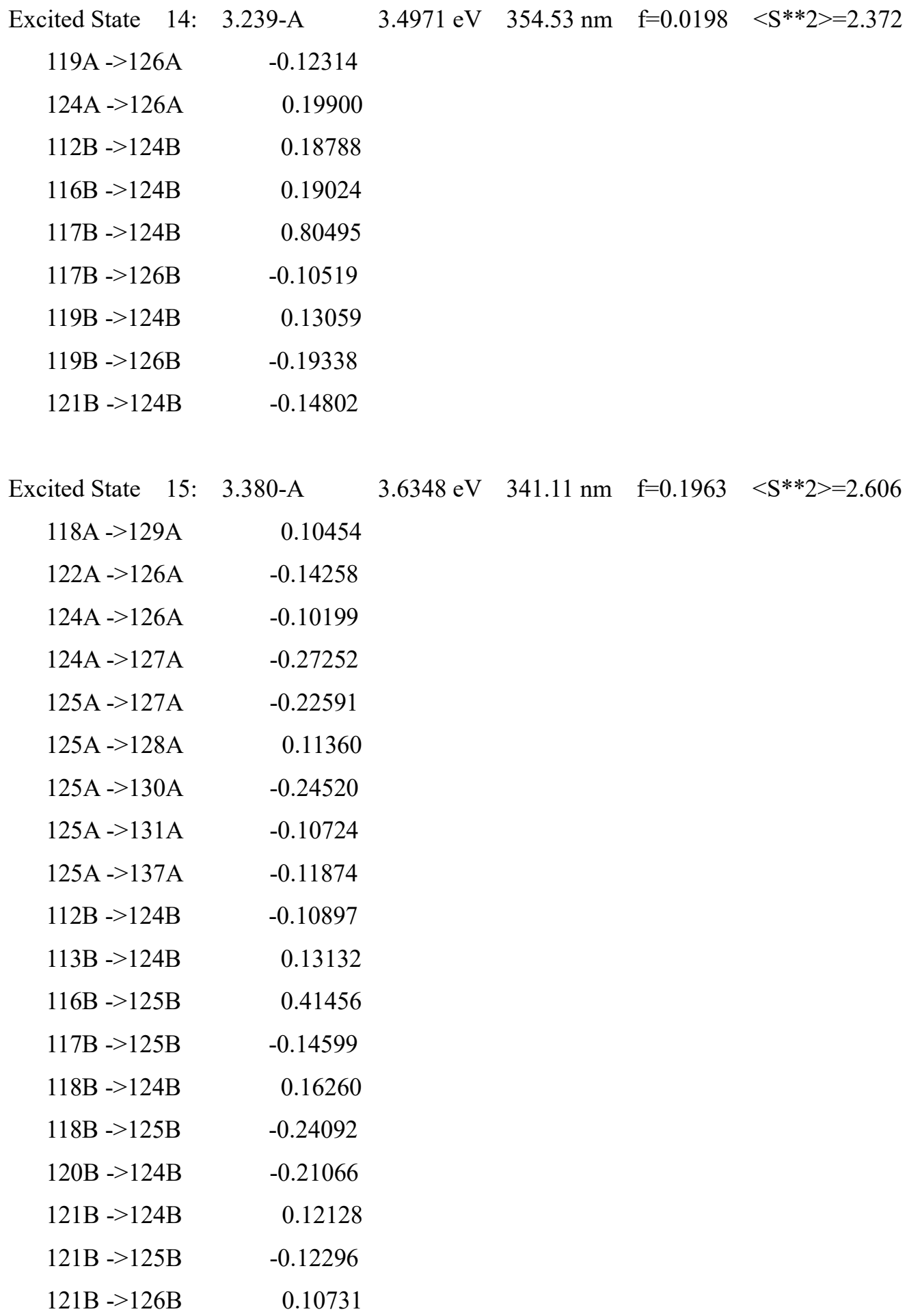


$$
\begin{array}{cc}
122 \mathrm{~B}->125 \mathrm{~B} & -0.16133 \\
123 \mathrm{~B}->124 \mathrm{~B} & -0.12694 \\
123 \mathrm{~B}->129 \mathrm{~B} & 0.10518
\end{array}
$$

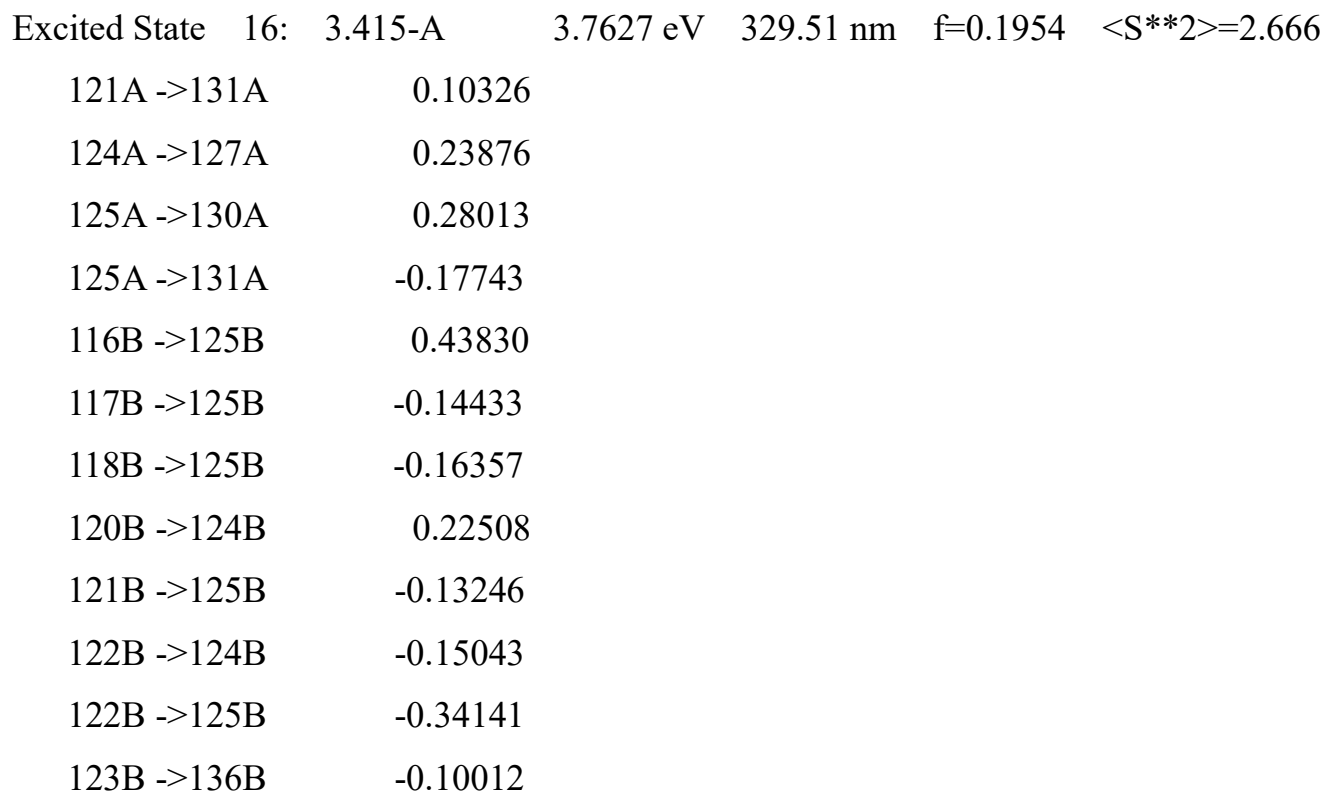

Excited State $\quad 17: \quad 3.349-\mathrm{A} \quad 3.8322 \mathrm{eV} \quad 323.53 \mathrm{~nm} \quad \mathrm{f}=0.0868 \quad<\mathrm{S} * * 2>=2.555$

$\begin{array}{lr}124 \mathrm{~A}->126 \mathrm{~A} & 0.20967 \\ 124 \mathrm{~A}->127 \mathrm{~A} & 0.14629 \\ 125 \mathrm{~A}->126 \mathrm{~A} & 0.13220 \\ 125 \mathrm{~A}->127 \mathrm{~A} & -0.10737 \\ 125 \mathrm{~A}->128 \mathrm{~A} & 0.17174 \\ 125 \mathrm{~A}->130 \mathrm{~A} & -0.19576 \\ 125 \mathrm{~A}->131 \mathrm{~A} & 0.30883 \\ 125 \mathrm{~A}->132 \mathrm{~A} & -0.13640 \\ 125 \mathrm{~A}->137 \mathrm{~A} & -0.13968 \\ 125 \mathrm{~A}->149 \mathrm{~A} & -0.15517 \\ 125 \mathrm{~A}->151 \mathrm{~A} & -0.15030 \\ 110 \mathrm{~B}->125 \mathrm{~B} & -0.14995 \\ 111 \mathrm{~B}->124 \mathrm{~B} & -0.21853 \\ 111 \mathrm{~B}->125 \mathrm{~B} & 0.11854 \\ 112 \mathrm{~B}->124 \mathrm{~B} & 0.16915 \\ 116 \mathrm{~B}->124 \mathrm{~B} & -0.10516 \\ 118 \mathrm{~B}->124 \mathrm{~B} & -0.24306 \\ 118 \mathrm{~B}->127 \mathrm{~B} & -0.11481 \\ 121 \mathrm{~B}->124 \mathrm{~B} & -0.20326 \\ 122 \mathrm{~B}->124 \mathrm{~B} & -0.22118\end{array}$


$123 \mathrm{~B}->125 \mathrm{~B} \quad 0.17603$

Excited State $\quad 18: \quad 3.454-\mathrm{A} \quad 3.8626 \mathrm{eV} \quad 320.99 \mathrm{~nm} \quad \mathrm{f}=0.0163 \quad<\mathrm{S} * * 2>=2.732$

$\begin{array}{lr}116 \mathrm{~A}->130 \mathrm{~A} & 0.11527 \\ 116 \mathrm{~A}->131 \mathrm{~A} & 0.13515 \\ 121 \mathrm{~A}->130 \mathrm{~A} & -0.10505 \\ 123 \mathrm{~A}->131 \mathrm{~A} & -0.16968 \\ 124 \mathrm{~A}->126 \mathrm{~A} & -0.13195 \\ 124 \mathrm{~A}->127 \mathrm{~A} & 0.11244 \\ 125 \mathrm{~A}->128 \mathrm{~A} & 0.15556 \\ 125 \mathrm{~A}->130 \mathrm{~A} & -0.17804 \\ 125 \mathrm{~A}->131 \mathrm{~A} & 0.53727 \\ 125 \mathrm{~A}->132 \mathrm{~A} & -0.23765 \\ 125 \mathrm{~A}->137 \mathrm{~A} & -0.22274 \\ 111 \mathrm{~B}->124 \mathrm{~B} & 0.13836 \\ 112 \mathrm{~B}->124 \mathrm{~B} & -0.12291 \\ 114 \mathrm{~B}->124 \mathrm{~B} & -0.12677 \\ 116 \mathrm{~B}->131 \mathrm{~B} & -0.11335 \\ 118 \mathrm{~B}->124 \mathrm{~B} & 0.20302 \\ 120 \mathrm{~B}->124 \mathrm{~B} & 0.11298 \\ 121 \mathrm{~B}->124 \mathrm{~B} & 0.15904 \\ 123 \mathrm{~B}->136 \mathrm{~B} & -0.13424\end{array}$

Excited State $\quad 19: \quad 3.408-\mathrm{A} \quad 3.9181 \mathrm{eV} \quad 316.44 \mathrm{~nm} \quad \mathrm{f}=0.1333 \quad<\mathrm{S} * * 2>=2.653$ $116 \mathrm{~A}->130 \mathrm{~A} \quad-0.10739$

$123 \mathrm{~A}->131 \mathrm{~A} \quad 0.11339$

$124 \mathrm{~A}->126 \mathrm{~A} \quad-0.33096$

$124 \mathrm{~A}->127 \mathrm{~A} \quad 0.17362$

$125 \mathrm{~A}->126 \mathrm{~A} \quad-0.12079$

$125 \mathrm{~A}->149 \mathrm{~A} \quad-0.12796$

$125 \mathrm{~A}->151 \mathrm{~A} \quad-0.13071$

$110 \mathrm{~B}->124 \mathrm{~B} \quad 0.18965$

$111 \mathrm{~B}->124 \mathrm{~B} \quad 0.40113$

$111 \mathrm{~B}->125 \mathrm{~B} \quad 0.12618$

$112 \mathrm{~B}->124 \mathrm{~B} \quad-0.16008$

$113 \mathrm{~B}->124 \mathrm{~B} \quad 0.16554$

$117 \mathrm{~B}->124 \mathrm{~B} \quad 0.11133$

$120 \mathrm{~B}->124 \mathrm{~B} \quad 0.15267$

$121 \mathrm{~B}->124 \mathrm{~B} \quad 0.26495$ 


$$
122 \mathrm{~B}->124 \mathrm{~B} \quad 0.11960
$$

$\begin{array}{rrrr}\text { Excited State 20: } & 3.377-\mathrm{A} & 3.9756 \mathrm{eV} & 311.87 \mathrm{~nm} \quad \mathrm{f}=0.2170 \quad<\mathrm{S} * * 2>=2.601 \\ 116 \mathrm{~A}->131 \mathrm{~A} & 0.11339 & 0.11723 & \\ 123 \mathrm{~A}->128 \mathrm{~A} & -0.10732 & \\ 124 \mathrm{~A}->127 \mathrm{~A} & -0.13547 \\ 125 \mathrm{~A}->126 \mathrm{~A} & 0.17423 \\ 125 \mathrm{~A}->127 \mathrm{~A} & -0.26862 \\ 125 \mathrm{~A}->128 \mathrm{~A} & 0.29349 \\ 125 \mathrm{~A}->130 \mathrm{~A} & 0.43298 \\ 125 \mathrm{~A}->131 \mathrm{~A} & -0.13612 \\ 125 \mathrm{~A}->132 \mathrm{~A} & -0.14074 \\ 125 \mathrm{~A}->133 \mathrm{~A} & 0.12140 \\ 125 \mathrm{~A}->134 \mathrm{~A} & -0.15205 \\ 125 \mathrm{~A}->135 \mathrm{~A} & 0.31454 \\ 125 \mathrm{~A}->137 \mathrm{~A} & 0.11081 \\ 125 \mathrm{~A}->138 \mathrm{~A} & 0.22267 \\ 116 \mathrm{~B}->125 \mathrm{~B} & -0.13409 \\ 120 \mathrm{~B}->124 \mathrm{~B} & 0.14697 \\ 122 \mathrm{~B}->125 \mathrm{~B} & 0.11022 \\ 123 \mathrm{~B}->130 \mathrm{~B} & -0.13216 \\ 123 \mathrm{~B}->131 \mathrm{~B} & \end{array}$

Table S11. Standard orientation of the optimized geometry for the closed-shell quinoidal form of the ring-opening form of 2 .

\begin{tabular}{ccccc}
\hline Tag & Symbol & \multicolumn{3}{c}{ Coordinates (Angstroms) } \\
\hline 1 & $\mathrm{C}$ & -1.7495280 & $\mathrm{Y}$ & $\mathrm{Z}$ \\
\hline 2 & $\mathrm{C}$ & 0.2233000 & 3.3814160 & -0.3381450 \\
3 & $\mathrm{C}$ & -0.4992260 & 5.7098650 & 0.2963550 \\
4 & $\mathrm{C}$ & -2.0352340 & 3.9714550 & 0.1845470 \\
5 & $\mathrm{C}$ & -1.0998090 & 2.9685420 & -0.4742000 \\
6 & $\mathrm{C}$ & 0.4466430 & 4.7766530 & -0.0884810 \\
7 & $\mathrm{C}$ & 1.3479910 & 2.5019640 & 0.4976970 \\
8 & $\mathrm{C}$ & 3.7027040 & 0.8765150 & 0.3626530 \\
9 & $\mathrm{C}$ & 1.4884230 & 1.3775400 & -0.4919910
\end{tabular}




\begin{tabular}{|c|c|c|c|c|}
\hline 10 & $\mathrm{C}$ & 2.4477540 & 2.8010670 & 1.2483020 \\
\hline 11 & $\mathrm{C}$ & 3.5502890 & 2.0211320 & 1.3170740 \\
\hline 12 & $\mathrm{C}$ & 2.6081590 & 0.6113960 & -0.4505660 \\
\hline 13 & $\mathrm{O}$ & 2.7168810 & -0.4405200 & -1.3086130 \\
\hline 14 & $\mathrm{C}$ & 3.8375520 & -1.2118020 & -1.2642260 \\
\hline 15 & $\mathrm{C}$ & 4.8649240 & -0.9200960 & -0.3508630 \\
\hline 16 & $\mathrm{~N}$ & 4.7856820 & 0.1497430 & 0.5152120 \\
\hline 17 & $\mathrm{C}$ & 5.9989430 & -1.7452050 & -0.3398220 \\
\hline 18 & $\mathrm{C}$ & 6.0950530 & -2.8160800 & -1.2069720 \\
\hline 19 & $\mathrm{C}$ & 5.0575960 & -3.0831070 & -2.1070730 \\
\hline 20 & $\mathrm{C}$ & 3.9259170 & -2.2831580 & -2.1412470 \\
\hline 21 & $\mathrm{C}$ & -1.5760490 & 1.6374240 & 0.0198110 \\
\hline 22 & $\mathrm{~N}$ & -2.7787090 & 1.2578970 & -0.5191590 \\
\hline 23 & $\mathrm{C}$ & -2.9035900 & -0.0157890 & -0.1929610 \\
\hline 24 & $\mathrm{C}$ & -1.7523860 & -0.3985120 & 0.6185510 \\
\hline 25 & $\mathrm{~N}$ & -0.9499510 & 0.6467560 & 0.7221170 \\
\hline 26 & $\mathrm{C}$ & -4.0469230 & -0.8053600 & -0.6695660 \\
\hline 27 & $\mathrm{C}$ & -6.2452010 & -2.2291310 & -1.6665990 \\
\hline 28 & $\mathrm{C}$ & -3.9397730 & -2.1688600 & -0.9654730 \\
\hline 29 & $\mathrm{C}$ & -5.2710010 & -0.1644860 & -0.8973900 \\
\hline 30 & $\mathrm{C}$ & -6.3597520 & -0.8703280 & -1.3864280 \\
\hline 31 & $\mathrm{C}$ & -5.0306380 & -2.8731670 & -1.4586630 \\
\hline 32 & $\mathrm{C}$ & -1.4467000 & -1.6482900 & 1.3268820 \\
\hline 33 & $\mathrm{C}$ & -0.7823870 & -3.9686760 & 2.7532270 \\
\hline 34 & $\mathrm{C}$ & -0.1082760 & -2.0134440 & 1.5184620 \\
\hline 35 & $\mathrm{C}$ & -2.4459670 & -2.4610490 & 1.8745810 \\
\hline 36 & $\mathrm{C}$ & -2.1149550 & -3.6103500 & 2.5802110 \\
\hline 37 & $\mathrm{C}$ & 0.2201180 & -3.1631490 & 2.2212950 \\
\hline 38 & $\mathrm{H}$ & -2.4900970 & 6.0433700 & -0.6114820 \\
\hline 39 & $\mathrm{H}$ & -0.2797430 & 6.7662220 & 0.2997030 \\
\hline 40 & $\mathrm{H}$ & -3.0026570 & 3.6317970 & -0.8230470 \\
\hline 41 & $\mathrm{H}$ & 1.4295390 & 5.1112100 & 0.8085040 \\
\hline 42 & $\mathrm{H}$ & 0.7108730 & 1.1287890 & -1.2005730 \\
\hline 43 & $\mathrm{H}$ & 2.3493830 & 3.6363680 & 1.9307960 \\
\hline 44 & $\mathrm{H}$ & 4.3538160 & 2.2181440 & 2.0175850 \\
\hline 45 & $\mathrm{H}$ & 6.7847810 & -1.5080940 & 0.3688580 \\
\hline 46 & $\mathrm{H}$ & 6.9746650 & -3.4504430 & -1.1916780 \\
\hline 47 & $\mathrm{H}$ & 5.1346590 & -3.9249590 & -2.7872040 \\
\hline 48 & $\mathrm{H}$ & 3.1085530 & -2.4712220 & -2.8281430 \\
\hline
\end{tabular}




\begin{tabular}{lllll}
49 & $\mathrm{H}$ & -7.0977740 & -2.7817830 & -2.0495470 \\
50 & $\mathrm{H}$ & -2.9925950 & -2.6764550 & -0.8216390 \\
51 & $\mathrm{H}$ & -5.3456840 & 0.8962080 & -0.6856980 \\
52 & $\mathrm{H}$ & -7.3035230 & -0.3589230 & -1.5506670 \\
53 & $\mathrm{H}$ & -4.9279510 & -3.9295570 & -1.6879290 \\
54 & $\mathrm{H}$ & -0.5259390 & -4.8692010 & 3.3029650 \\
55 & $\mathrm{H}$ & 0.6650110 & -1.3764030 & 1.1034280 \\
56 & $\mathrm{H}$ & -3.4871690 & -2.1811520 & 1.7609360 \\
57 & $\mathrm{H}$ & -2.9035100 & -4.2248120 & 3.0040270 \\
58 & $\mathrm{H}$ & 1.2633280 & -3.4339220 & 2.3546390 \\
\hline
\end{tabular}

SCF Done: E(RCAM-B3LYP)

$=-1508.75919526 \quad$ A.U.

Zero-point correction

$=0.457344($ Hartree $/$ Particle $)$

Thermal correction to Energy

$=0.484295$

Thermal correction to Enthalpy

$=0.485240$

Thermal correction to Gibbs Free Energy

$=0.397098$

Sum of electronic and zero-point Energies

$=-1508.301851$

Sum of electronic and thermal Energies

$=-1508.274900$

Sum of electronic and thermal Enthalpies

$=-1508.273956$

Sum of electronic and thermal Free Energies

$=-1508.362098$

$\begin{array}{lllllll}\text { Low frequencies --- } & -0.9370 & -0.8699 & -0.0009 & -0.0006 & -0.0005 & 1.1605\end{array}$

Low frequencies --- $\quad 10.0162 \quad 17.5067 \quad 31.4979$

The Results of the TDDFT calculation

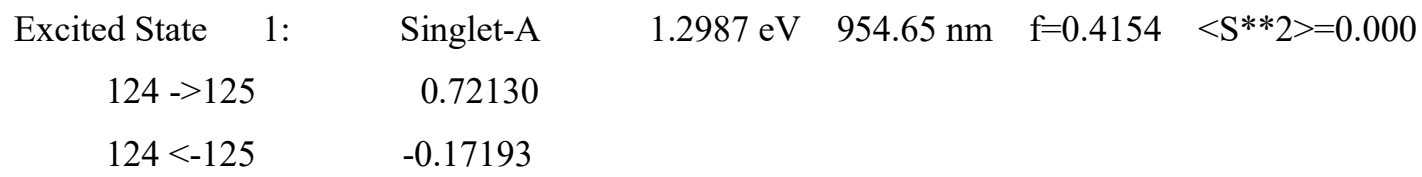

This state for optimization and/or second-order correction.

Total Energy, E(TD-HF/TD-DFT) = -1508.76070796

Copying the excited state density for this state as the 1-particle RhoCI density.

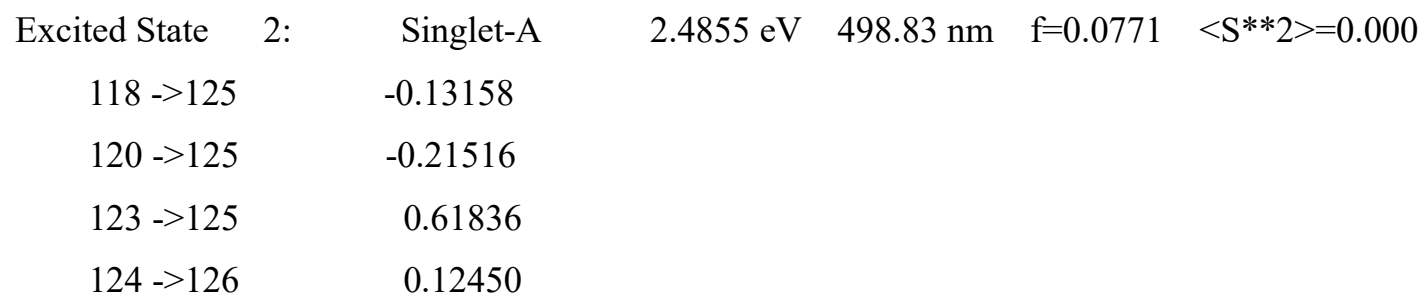



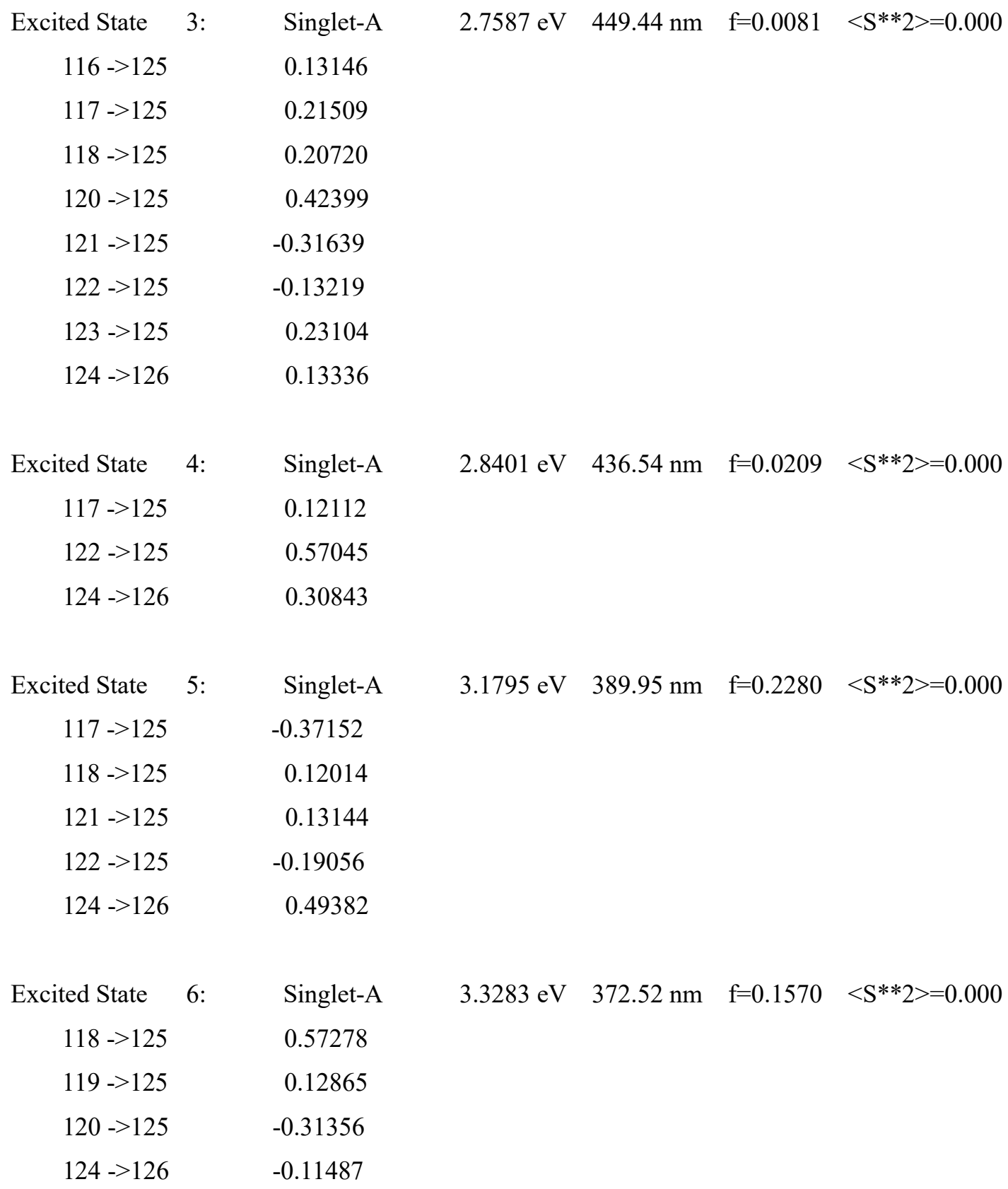

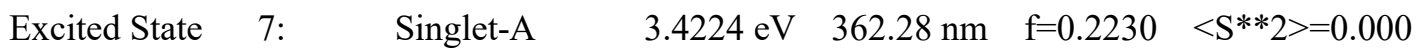

$\begin{array}{lr}117->125 & -0.27826 \\ 118->125 & 0.17164 \\ 119->125 & -0.19373 \\ 120->125 & 0.32996 \\ 121->125 & 0.26816 \\ 122->125 & 0.23508 \\ 123->125 & 0.12641 \\ 124->126 & -0.26488\end{array}$




\begin{tabular}{|c|c|c|}
\hline Excited State & Singlet-A & $3.5084 \mathrm{eV} \quad 353.39 \mathrm{~nm} \quad \mathrm{f}=0.0549$ \\
\hline $117->125$ & 0.39244 & \\
\hline $118->125$ & 0.10130 & \\
\hline $119->125$ & -0.29829 & \\
\hline $121->125$ & 0.43815 & \\
\hline $124->126$ & 0.12835 & \\
\hline
\end{tabular}

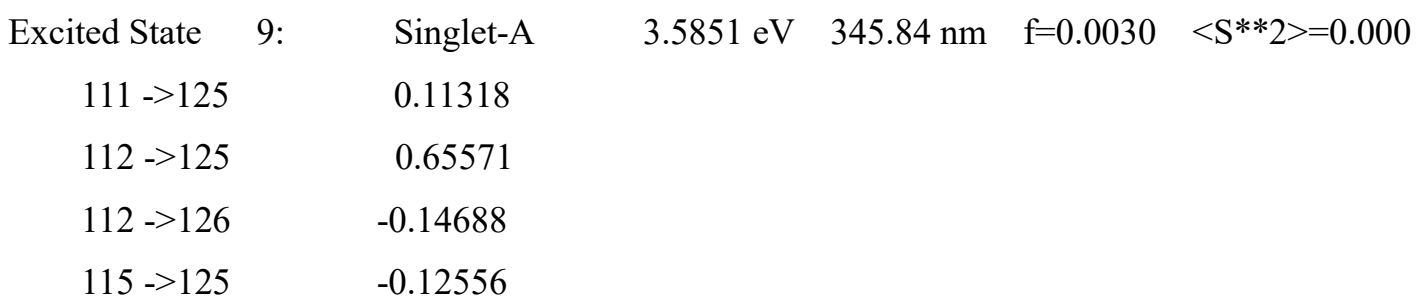

Excited State $\quad 10: \quad$ Singlet-A $\quad 3.6996 \mathrm{eV} \quad 335.13 \mathrm{~nm} \quad \mathrm{f}=0.0151 \quad<\mathrm{S} * * 2>=0.000$

$\begin{array}{ll}111->125 & 0.15676 \\ 113->125 & 0.10004 \\ 116->125 & 0.45156 \\ 119->125 & 0.39900 \\ 121->125 & 0.20357\end{array}$

Excited State 11: $\quad$ Singlet-A $\quad 3.7917 \mathrm{eV} \quad 326.99 \mathrm{~nm} \quad \mathrm{f}=0.0245 \quad<\mathrm{S}^{* *} 2>=0.000$

$\begin{array}{lr}116->125 & -0.41086 \\ 119->125 & 0.42168 \\ 120->125 & 0.18272 \\ 121->125 & 0.21723 \\ 124->127 & -0.12088\end{array}$

Excited State 12: $\quad$ Singlet-A $\quad 3.8897 \mathrm{eV} \quad 318.75 \mathrm{~nm} \quad \mathrm{f}=0.3912 \quad<\mathrm{S} * * 2>=0.000$

$\begin{array}{lr}113->125 & -0.19462 \\ 122->125 & -0.12482 \\ 124->127 & 0.62428\end{array}$

Excited State 13: $\quad$ Singlet-A $\quad 4.0125 \mathrm{eV} \quad 308.99 \mathrm{~nm} \quad \mathrm{f}=0.0090 \quad<\mathrm{S} * * 2>=0.000$

$\begin{array}{rr}111->125 & -0.24757 \\ 113->125 & 0.48624 \\ 114->125 & 0.18300 \\ 115->125 & 0.21730 \\ 116->125 & -0.10872 \\ 117->125 & 0.13633\end{array}$




$\begin{array}{cc}118->125 & -0.12093 \\ 124->129 & 0.13272\end{array}$

Excited State 14: Singlet-A

$4.1232 \mathrm{eV} \quad 300.70 \mathrm{~nm} \quad \mathrm{f}=0.1442 \quad<\mathrm{S} * * 2>=0.000$

$124->128$

0.67542

$\begin{array}{cc}\text { Excited State } 15: & \text { Singlet-A } \\ 113->125 & -0.19947 \\ 114->125 & 0.62240 \\ 124->127 & -0.11240\end{array}$

Excited State 16: Singlet-A

$115->125$

0.13363

$124->129$

$-0.22324$

$124->130$

$-0.17571$

$124->131$

0.57528
$4.3668 \mathrm{eV} \quad 283.92 \mathrm{~nm} \quad \mathrm{f}=0.0624 \quad<\mathrm{S} * * 2>=0.000$

$4.2216 \mathrm{eV} \quad 293.69 \mathrm{~nm} \quad \mathrm{f}=0.0323<\mathrm{S} * * 2>=0.000$

$4.3668 \mathrm{eV} 283.92 \mathrm{~nm}$ f $=0.0624<\mathrm{S} * 2>0.000$

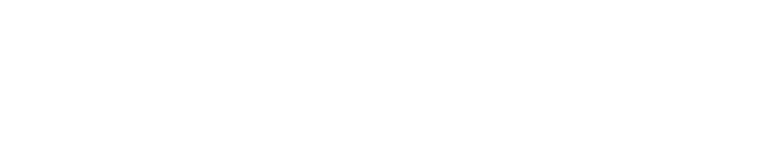

$4.4108 \mathrm{eV} \quad 281.09 \mathrm{~nm} \quad \mathrm{f}=0.0367 \quad<\mathrm{S} * * 2>=0.000$

Excited State 18: $\quad$ Singlet-A $\quad 4.4609 \mathrm{eV} \quad 277.94 \mathrm{~nm} \quad \mathrm{f}=0.0130 \quad<\mathrm{S} * * 2>=0.000$

$\begin{array}{ll}111->125 & 0.51688 \\ 113->125 & 0.13224 \\ 114->125 & 0.15479 \\ 115->125 & 0.19419 \\ 116->125 & -0.19669 \\ 122->125 & -0.11503 \\ 124->129 & -0.19054\end{array}$

$\begin{array}{cllll}\text { Excited State } \quad \text { 19: } & \text { Singlet-A } & 4.5362 \mathrm{eV} & 273.32 \mathrm{~nm} & \mathrm{f}=0.0655 \quad<\mathrm{S} * * 2>=0.000 \\ 111->125 & 0.22706 & & & \\ 124->129 & 0.53529 & & & \\ 124->131 & 0.18829 & & \end{array}$ 


\begin{tabular}{|c|c|c|c|}
\hline Excited State 20: & Singlet-A & $4.6087 \mathrm{eV}$ & $269.02 \mathrm{~nm} \quad \mathrm{f}=0.1036$ \\
\hline $115->125$ & -0.13331 & & \\
\hline $123->126$ & -0.11040 & & \\
\hline $124->130$ & 0.37335 & & \\
\hline $124->131$ & 0.24277 & & \\
\hline $124->132$ & 0.19693 & & \\
\hline $124->133$ & -0.31202 & & \\
\hline $124->134$ & -0.22855 & & \\
\hline
\end{tabular}

\section{Singlet biradical}

Quinoidal

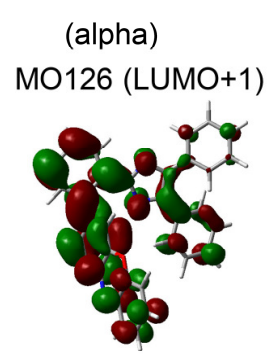

(beta)

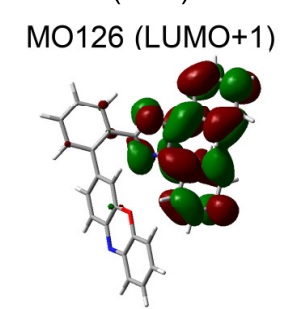

MO130 (LUMO+1)
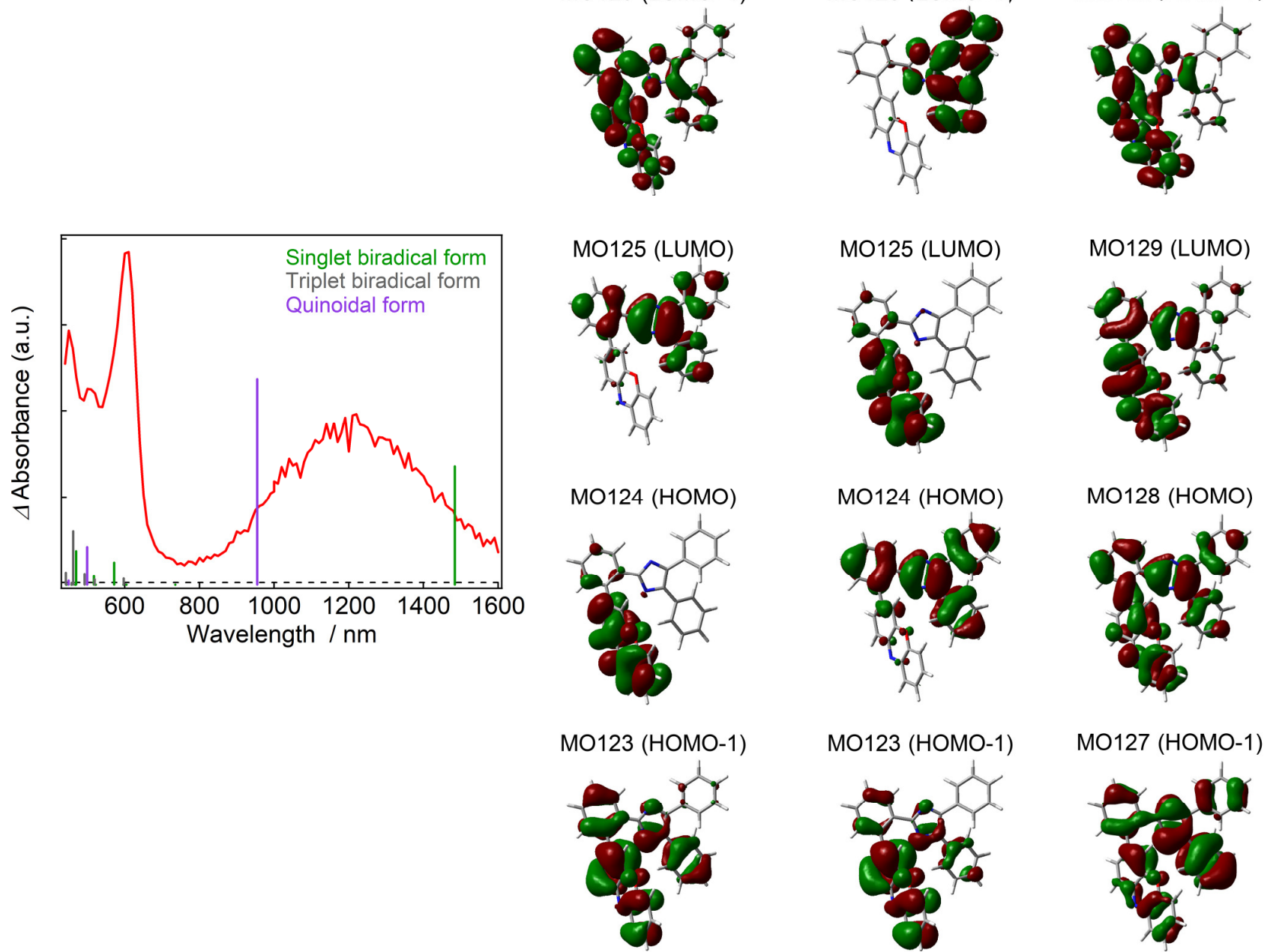

MO127 (HOMO-1)

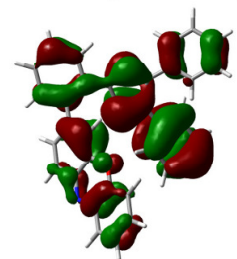

Figure S52. (a) UV-vis absorption spectrum of 2 in benzene at room temperature. The calculated absorption spectrum ((U)CAM-B3LYP/6-31G+(d)// (U)CAM-B3LYP /6-31G(d) level of the theory) is shown by the blue 
vertical lines. (b) The relevant molecular orbitals of the 2 calculated at the (U)CAM-B3LYP /6-31G(d) level of the theory.

Table S12. Standard orientation of the optimized geometry for the 1c.

\begin{tabular}{|c|c|c|c|c|}
\hline \multirow{2}{*}{ Tag } & \multirow{2}{*}{ Symbol } & \multicolumn{3}{|c|}{ Coordinates (Angstroms) } \\
\hline & & $\mathrm{X}$ & $\mathrm{Y}$ & $\mathrm{Z}$ \\
\hline 1 & $\mathrm{C}$ & 4.2514930 & -1.5135070 & 0.1882950 \\
\hline 2 & $\mathrm{C}$ & 3.1642350 & 0.6738910 & -0.0693410 \\
\hline 3 & $\mathrm{C}$ & 5.5852890 & 0.5109460 & -0.0401660 \\
\hline 4 & $\mathrm{C}$ & 4.4512840 & 1.2751770 & -0.1333150 \\
\hline 5 & $\mathrm{C}$ & 5.4811650 & -0.8865380 & 0.1218760 \\
\hline 6 & $\mathrm{C}$ & 3.0842560 & -0.7466780 & 0.0924910 \\
\hline 7 & $\mathrm{~S}$ & 1.5683080 & -1.5557570 & 0.1691230 \\
\hline 8 & $\mathrm{C}$ & 0.4215450 & -0.2851610 & 0.0204040 \\
\hline 9 & $\mathrm{C}$ & 0.8526680 & 1.0852280 & -0.1177390 \\
\hline 10 & $\mathrm{~N}$ & 2.1090820 & 1.4969130 & -0.1627370 \\
\hline 11 & $\mathrm{C}$ & -0.1640790 & 2.0863570 & -0.2163120 \\
\hline 12 & $\mathrm{C}$ & -1.4834190 & 1.7552710 & -0.1771980 \\
\hline 13 & $\mathrm{C}$ & -1.9060500 & 0.3948550 & -0.0465640 \\
\hline 14 & $\mathrm{C}$ & -0.9312480 & -0.6027670 & 0.0507540 \\
\hline 15 & $\mathrm{C}$ & -3.3276640 & 0.0600260 & -0.0193470 \\
\hline 16 & $\mathrm{C}$ & -6.0523120 & -0.5820640 & 0.0505810 \\
\hline 17 & $\mathrm{C}$ & -4.2662210 & 0.9699330 & 0.5011850 \\
\hline 18 & $\mathrm{C}$ & -3.7863680 & -1.1773800 & -0.5076110 \\
\hline 19 & $\mathrm{C}$ & -5.1360320 & -1.4896910 & -0.4799190 \\
\hline 20 & $\mathrm{C}$ & -5.6131540 & 0.6451030 & 0.5445990 \\
\hline 21 & $\mathrm{H}$ & 4.1900310 & -2.5894850 & 0.3121840 \\
\hline 22 & $\mathrm{H}$ & 6.5636990 & 0.9742310 & -0.0895510 \\
\hline 23 & $\mathrm{H}$ & 4.4857440 & 2.3512470 & -0.2563900 \\
\hline 24 & $\mathrm{H}$ & 6.3827160 & -1.4848890 & 0.1956860 \\
\hline 25 & $\mathrm{H}$ & 0.1691540 & 3.1107010 & -0.3349080 \\
\hline 26 & $\mathrm{H}$ & -2.2321380 & 2.5303390 & -0.2864170 \\
\hline 27 & $\mathrm{H}$ & -1.2333650 & -1.6346480 & 0.1918810 \\
\hline 28 & $\mathrm{H}$ & -7.1078090 & -0.8302200 & 0.0763240 \\
\hline 29 & $\mathrm{H}$ & -3.9365590 & 1.9159820 & 0.9164940 \\
\hline 30 & $\mathrm{H}$ & -3.0890950 & -1.8771750 & -0.9558910 \\
\hline 31 & $\mathrm{H}$ & -5.4782590 & -2.4378990 & -0.8791780 \\
\hline
\end{tabular}


SCF Done: $\quad$ E(RmPW1PW91)

Zero-point correction

Thermal correction to Energy

Thermal correction to Enthalpy

Thermal correction to Gibbs Free Energy

Sum of electronic and zero-point Energies

Sum of electronic and thermal Energies

Sum of electronic and thermal Enthalpies

Sum of electronic and thermal Free Energies
$=$

$=$

$=$

$=$

$=$

$-1145.68822052$

A.U.

$=\quad-1145.437485$

$=\quad-1145.422868$

$=\quad-1145.421924$

$=$

$\begin{array}{lllllll}\text { Low frequencies --- } & -7.6964 & -4.4300 & 0.0021 & 0.0023 & 0.0029 & 5.7482\end{array}$

Low frequencies --- $\quad 34.1123 \quad 53.4016 \quad 81.2446$

The Result for the TDDFT calculation

Excited State 1: $\quad$ Singlet-A $\quad 2.2669 \mathrm{eV} \quad 546.94 \mathrm{~nm} \quad \mathrm{f}=0.3022 \quad<\mathrm{S}^{* *} 2>=0.000$

$$
\begin{array}{ll}
69->72 & 0.23776 \\
70->72 & -0.13299 \\
71->72 & 0.65121
\end{array}
$$

This state for optimization and/or second-order correction.

Total Energy, E(TD-HF/TD-DFT) $=-1145.61851618$

Copying the excited state density for this state as the 1-particle RhoCI density.

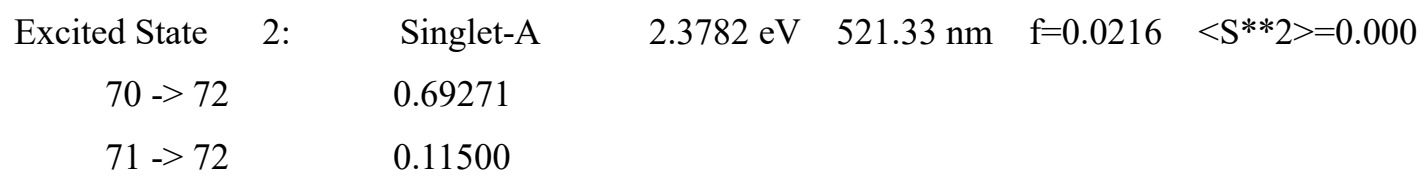

$\begin{array}{rllll}\text { Excited State } & 3: & \text { Singlet-A } & 2.5919 \mathrm{eV} & 478.35 \mathrm{~nm} \quad \mathrm{f}=0.3096 \quad<\mathrm{S} * * 2>=0.000 \\ 69->72 & & 0.65684 & \\ 71->72 & -0.24403 & \end{array}$
Excited State $\quad 4: \quad$ Singlet-A $\quad 2.9389 \mathrm{eV} \quad 421.87 \mathrm{~nm} \quad \mathrm{f}=0.0013 \quad<\mathrm{S} * * 2>=0.000$ $66->72 \quad 0.69604$

Excited State 5: $\quad$ Singlet-A $\quad 3.3487 \mathrm{eV} \quad 370.24 \mathrm{~nm} \quad \mathrm{f}=0.0032 \quad<\mathrm{S} * * 2>=0.000$ 


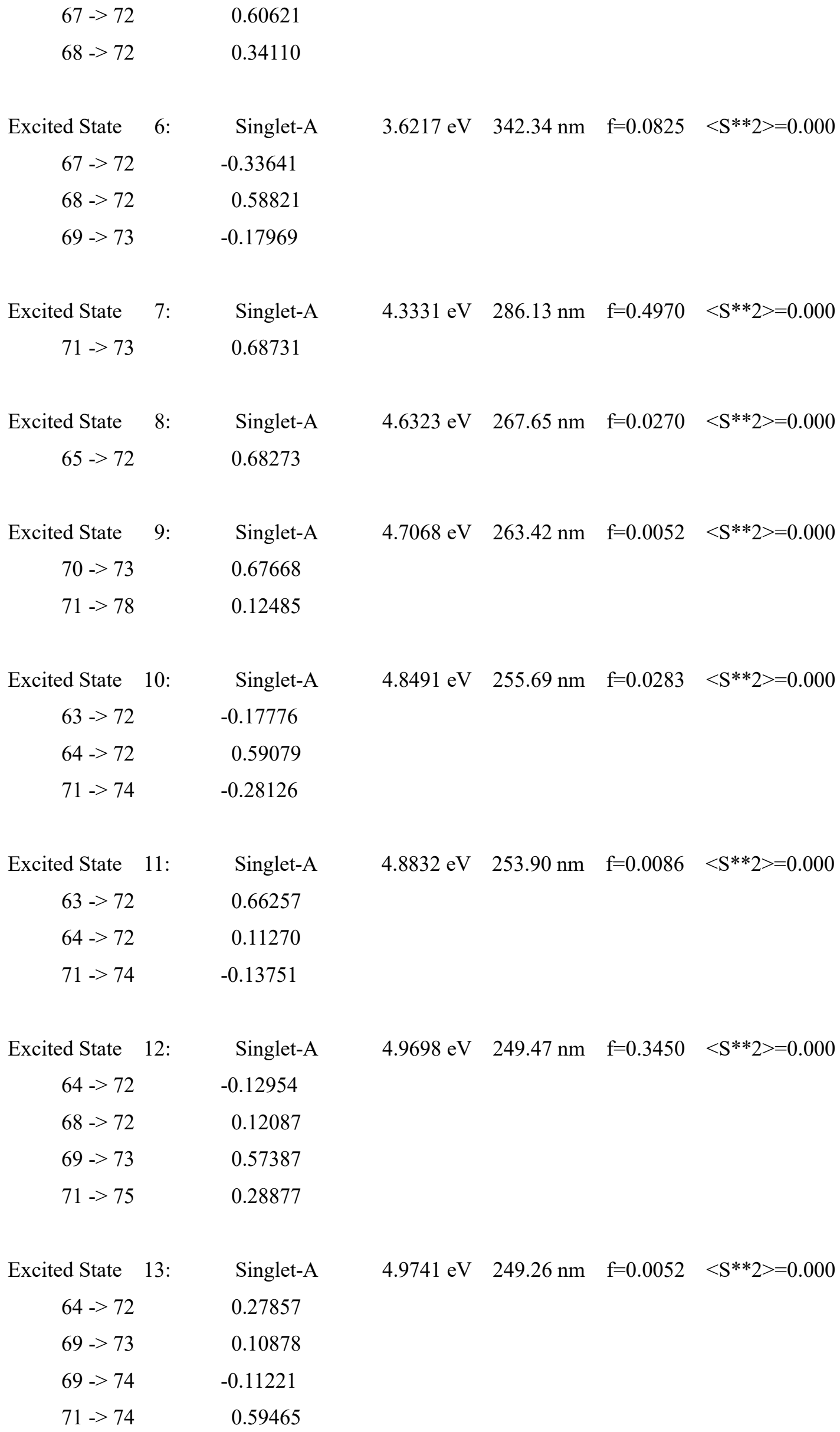



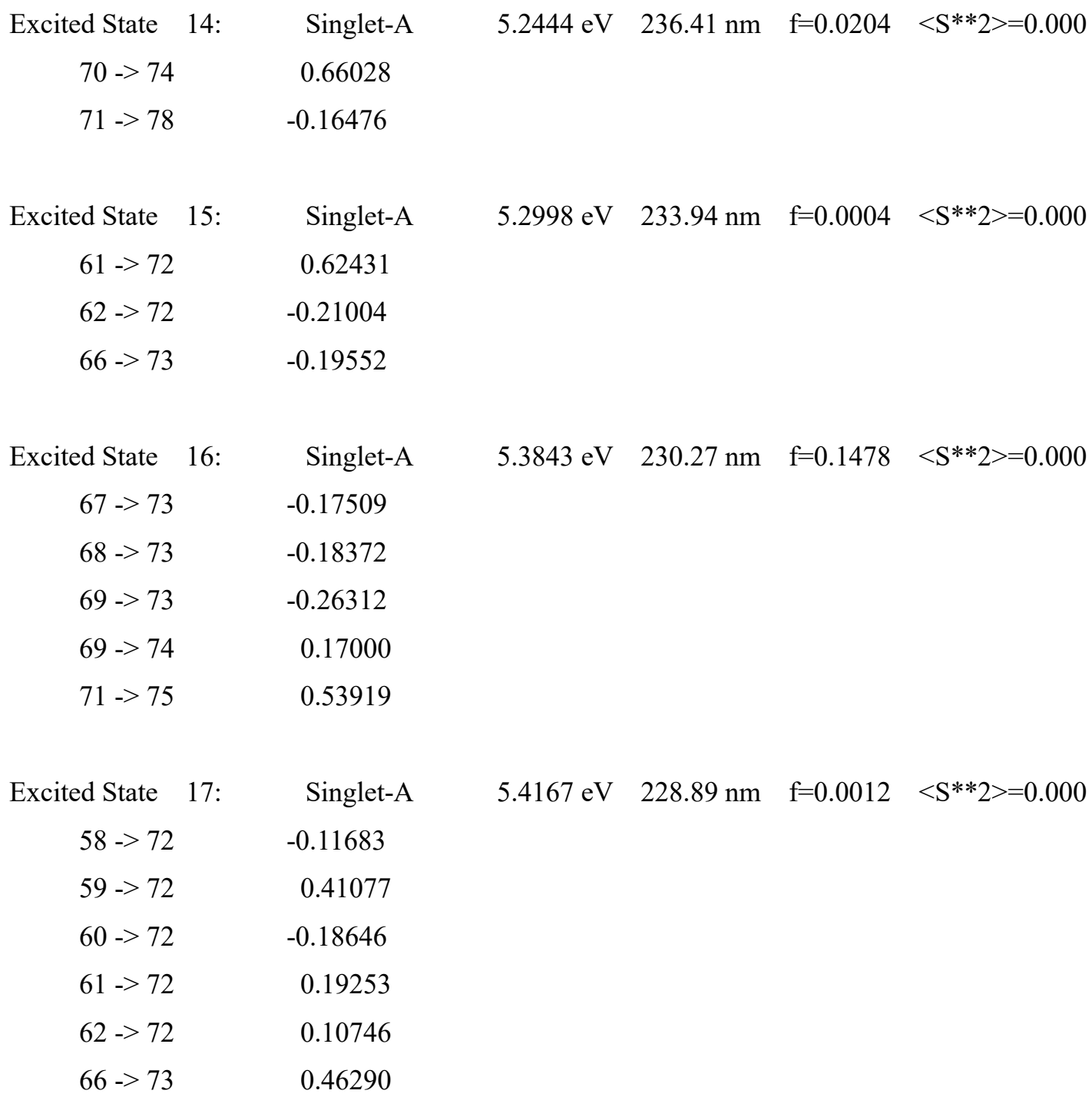

Excited State 18: $\quad$ Singlet-A $\quad 5.4490 \mathrm{eV} \quad 227.54 \mathrm{~nm} \quad \mathrm{f}=0.0022 \quad<\mathrm{S}^{* *} 2>=0.000$

$\begin{array}{lc}59-72 & -0.42260 \\ 60->72 & 0.16317 \\ 62->72 & -0.21851 \\ 66-73 & 0.47135\end{array}$

Excited State 19: $\quad$ Singlet-A $\quad 5.5485 \mathrm{eV} \quad 223.45 \mathrm{~nm} \quad \mathrm{f}=0.2676 \quad<\mathrm{S}^{* *} 2>=0.000$

$\begin{array}{ll}60->72 & -0.11481 \\ 62->72 & -0.11794 \\ 68->73 & -0.17991 \\ 69->74 & 0.59310 \\ 71->75 & -0.21089\end{array}$

Excited State $\quad 20: \quad$ Singlet-A $\quad 5.6637 \mathrm{eV} \quad 218.91 \mathrm{~nm} \quad \mathrm{f}=0.0216 \quad<\mathrm{S}^{* *} 2>=0.000$ 


$\begin{array}{lr}58->72 & -0.11621 \\ 59-72 & -0.24583 \\ 61-72 & 0.16028 \\ 62->72 & 0.52750 \\ 67->73 & -0.16736 \\ 69-74 & 0.12361 \\ 69-75 & -0.17065\end{array}$

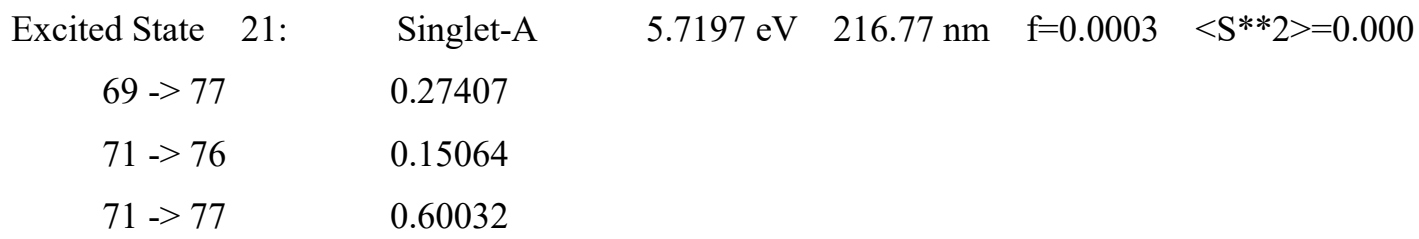

Excited State 22: $\quad$ Singlet-A $\quad 5.7456 \mathrm{eV} \quad 215.79 \mathrm{~nm} \quad \mathrm{f}=0.0033 \quad<\mathrm{S} * * 2>=0.000$ $58 \rightarrow 72 \quad 0.55020$

$70->74 \quad 0.10988$

$70 \rightarrow 75 \quad 0.21808$

$70->76 \quad 0.17781$

$71-76 \quad-0.11281$

$71 \rightarrow 78 \quad 0.21795$

Excited State 23: $\quad$ Singlet-A $\quad 5.7469 \mathrm{eV} \quad 215.74 \mathrm{~nm} \quad \mathrm{f}=0.0091 \quad<\mathrm{S} * * 2>=0.000$

$\begin{array}{lr}58->72 & 0.37643 \\ 69-75 & -0.12687 \\ 70-73 & 0.10466 \\ 70->74 & -0.15538 \\ 70-75 & -0.25837 \\ 70-76 & -0.24309 \\ 71->76 & 0.10021 \\ 71-78 & -0.32700\end{array}$

Excited State $\quad 24: \quad$ Singlet-A $\quad 5.7946 \mathrm{eV} \quad 213.96 \mathrm{~nm} \quad \mathrm{f}=0.0040 \quad<\mathrm{S}^{* *} 2>=0.000$

$\begin{array}{lr}59->72 & 0.15413 \\ 60->72 & 0.30514 \\ 68->73 & 0.39889 \\ 69-74 & 0.21201 \\ 69-75 & -0.30887 \\ 70->75 & 0.16096\end{array}$




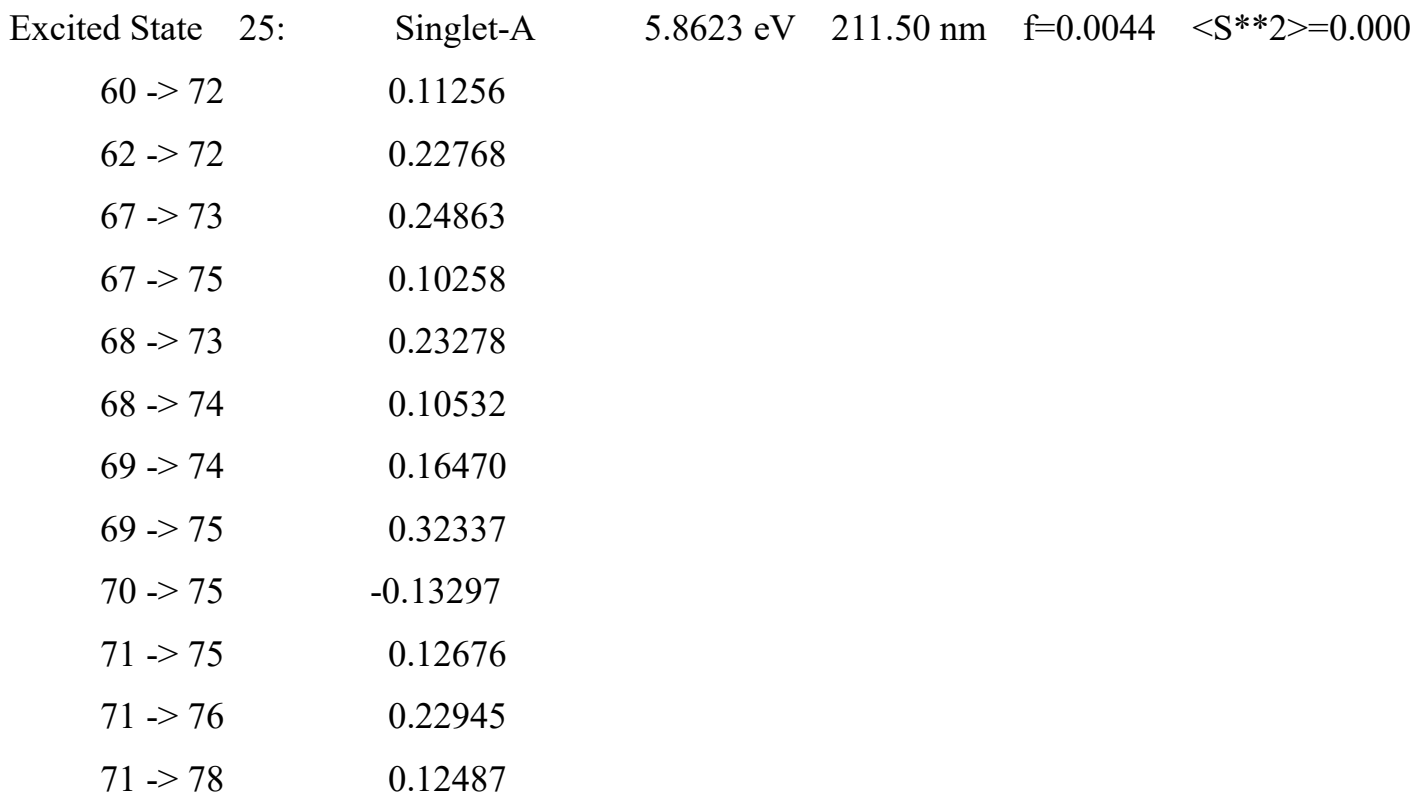

Excited State 26: $\quad$ Singlet-A $\quad 5.9382 \mathrm{eV} \quad 208.79 \mathrm{~nm} \quad \mathrm{f}=0.0077 \quad<$ S**2 $>=0.000$

$\begin{array}{lc}60-72 & -0.15001 \\ 70-75 & 0.54126 \\ 70->76 & -0.14633 \\ 70-78 & -0.13747 \\ 71-76 & 0.28534 \\ 71-78 & -0.14958\end{array}$

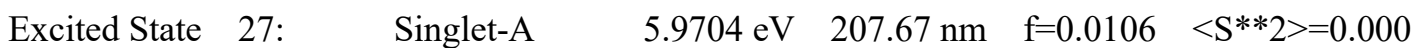

$\begin{array}{lc}59->72 & 0.12664 \\ 60->72 & 0.37311 \\ 68->73 & -0.11589 \\ 69->75 & 0.36093 \\ 70->75 & 0.19990 \\ 70->76 & -0.13185 \\ 71->76 & -0.26211 \\ 71-78 & -0.18662\end{array}$

Excited State 28: $\quad$ Singlet-A $\quad 6.0395 \mathrm{eV} \quad 205.29 \mathrm{~nm} \quad \mathrm{f}=0.0223 \quad<\mathrm{S} * * 2>=0.000$ 67 -> $73 \quad 0.46501$

$68->74 \quad 0.23589$

$69->75 \quad-0.20860$

$71 \rightarrow 75 \quad 0.10728$

$71-76 \quad-0.29423$

$71-78 \quad-0.16089$ 


$\begin{array}{cc}\text { Excited State } 29: & \text { Singlet-A } \\ 59->72 & -0.12093 \\ 60->72 & -0.33114 \\ 67->73 & -0.17082 \\ 67->74 & -0.12652 \\ 67->75 & 0.12387 \\ 68->73 & 0.35714 \\ 69->75 & 0.20617 \\ 70->78 & 0.17191 \\ 71->76 & -0.25074 \\ 71->78 & -0.10066\end{array}$

Excited State $30: \quad$ Singlet-A $\quad 6.2679 \mathrm{eV} \quad 197.81 \mathrm{~nm} \quad \mathrm{f}=0.0001 \quad<\mathrm{S}^{* *} 2>=0.000$

$\begin{array}{lr}57->72 & -0.20068 \\ 69->76 & 0.15739 \\ 69->77 & 0.55990 \\ 71-77 & -0.26874\end{array}$

Excited State $31: \quad$ Singlet-A $\quad 6.2775 \mathrm{eV} \quad 197.50 \mathrm{~nm} \quad \mathrm{f}=0.0004 \quad<\mathrm{S} * * 2>=0.000$

$\begin{array}{ll}57->72 & 0.62302 \\ 66->74 & 0.13786 \\ 69->77 & 0.18870\end{array}$

Excited State $32: \quad$ Singlet-A $\quad 6.3263 \mathrm{eV} \quad 195.98 \mathrm{~nm} \quad \mathrm{f}=0.0024 \quad<\mathrm{S} * * 2>=0.000$

$\begin{array}{lc}55-72 & 0.16668 \\ 57->72 & -0.12746 \\ 66->74 & 0.63956 \\ 66-75 & 0.11140\end{array}$

Excited State $33: \quad$ Singlet-A $\quad 6.3945 \mathrm{eV} \quad 193.89 \mathrm{~nm} \quad \mathrm{f}=0.0567 \quad<\mathrm{S}^{* *} 2>=0.000$

$\begin{array}{lr}56->72 & 0.53051 \\ 67->74 & -0.15647 \\ 67->75 & -0.14189 \\ 68->74 & 0.17918 \\ 68->75 & 0.19308 \\ 69-76 & -0.17443 \\ 70->76 & 0.11116 \\ 70-78 & -0.14674\end{array}$



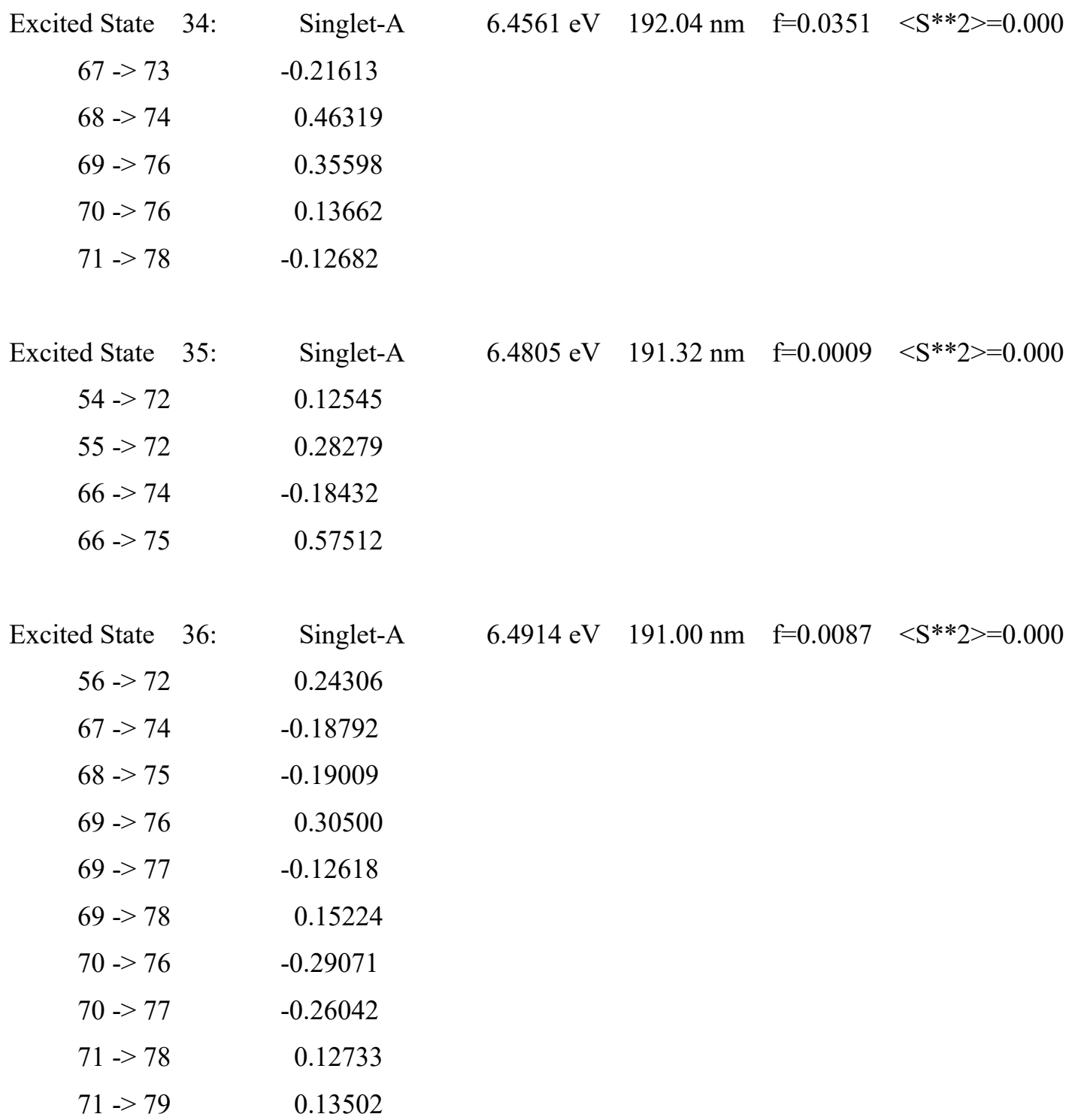

Excited State $\quad 37: \quad$ Singlet-A $\quad 6.5034 \mathrm{eV} \quad 190.65 \mathrm{~nm} \quad \mathrm{f}=0.0072 \quad<\mathrm{S}^{* *} 2>=0.000$

$\begin{array}{ll}56->72 & 0.13812 \\ 68->74 & -0.11323 \\ 68->75 & -0.10840 \\ 69->76 & 0.12598 \\ 70->77 & 0.60263 \\ 71->79 & 0.19350\end{array}$
Excited State $\quad 38: \quad$ Singlet-A $\quad 6.5169 \mathrm{eV} \quad 190.25 \mathrm{~nm} \quad \mathrm{f}=0.0252 \quad<\mathrm{S}^{* *} 2>=0.000$

$\begin{array}{lr}56->72 & -0.15382 \\ 68->74 & 0.20942 \\ 68->75 & 0.13776 \\ 69->76 & -0.13887\end{array}$




$$
71-79 \quad 0.55694
$$

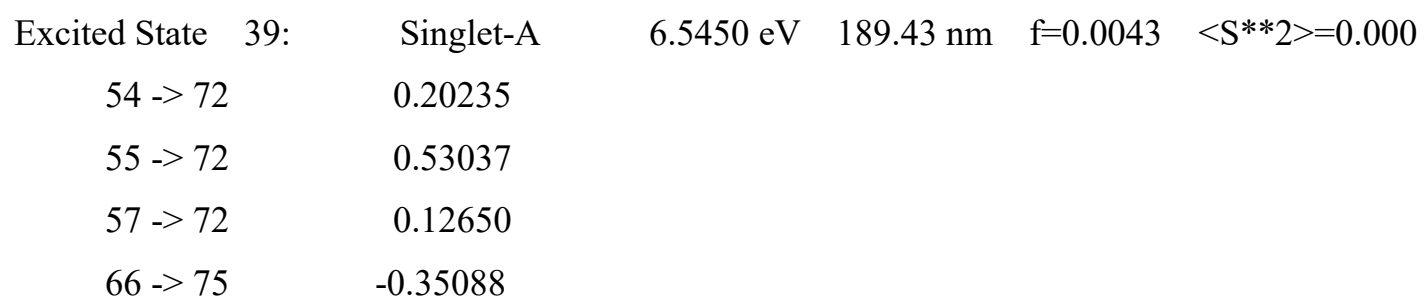

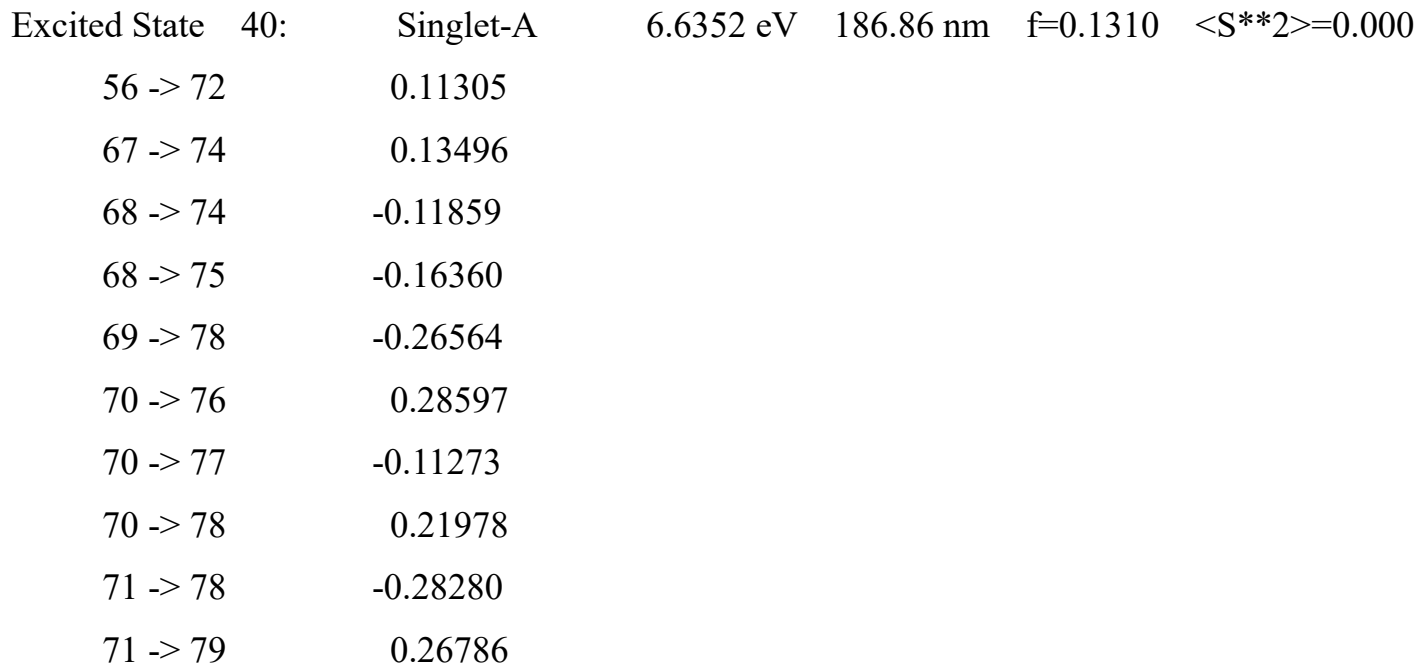

Table S13. Standard orientation of the optimized geometry for $1 \mathrm{a}$.

\begin{tabular}{ccccc}
\hline Tag & Symbol & \multicolumn{3}{c}{ Coordinates (Angstroms) } \\
\hline 1 & & $\mathrm{X}$ & $\mathrm{Y}$ & $\mathrm{Z}$ \\
\hline 2 & $\mathrm{C}$ & 1.4646930 & 5.2262400 & -0.3065970 \\
3 & $\mathrm{C}$ & 0.0742750 & 5.3236640 & -0.3203370 \\
4 & $\mathrm{C}$ & -0.6775080 & 4.1611850 & -0.2186670 \\
5 & $\mathrm{C}$ & -0.0869230 & 2.8931090 & -0.1170040 \\
6 & $\mathrm{C}$ & 1.3267520 & 2.7891720 & -0.1439340 \\
7 & $\mathrm{C}$ & 2.0682520 & 3.9829200 & -0.2211820 \\
8 & $\mathrm{C}$ & 2.0836630 & 1.5338850 & -0.1304280 \\
9 & $\mathrm{C}$ & -1.0083080 & 1.7468750 & 0.0851740 \\
10 & $\mathrm{~N}$ & 1.5684410 & 0.3380860 & -0.4675680 \\
11 & $\mathrm{C}$ & 2.6177550 & -0.5129480 & -0.3693830 \\
12 & $\mathrm{C}$ & 3.7541960 & 0.2259670 & 0.0565080 \\
13 & $\mathrm{~N}$ & 3.3968010 & 1.5255660 & 0.1809270 \\
14 & $\mathrm{C}$ & -2.1487020 & 1.6050250 & -0.7096810 \\
& $\mathrm{C}$ & -3.0623670 & 0.5789700 & -0.4890680 \\
& & $\mathrm{~S} 110$ & &
\end{tabular}


$-2.8896680$

$-1.7730830$

$-0.8429730$

$-4.4342320$

$-5.6216110$

$-5.1950130$

$-3.8336110$

$-6.9709820$

$-7.9292550$

$-7.5187620$

$-6.1661680$

5.1138990

2.4342040

1.1456580

0.9154540

1.9627700

3.2407920

3.4720760

6.1627300

7.4602860

7.7568550

6.7265270

5.4254550

$-3.4475440$

2.0786900

$-0.4180030$

$-1.7615580$

3.1480400

$-2.3109810$

$-1.6223470$

0.0261160

$-7.2678940$

$-8.9814360$

$-8.2492660$

$-5.8653190$

0.3328630

$-0.0926600$

1.7830160

4.0666550
$-0.3200510$

0.5656920

$-0.1529320$

1.3923370

0.8448970

1.1403650

0.3506140

$-1.5907310$

$-0.2287180$

$-0.4160260$

$-1.0697230$

0.6306410

$-1.3527440$

0.7767200

0.0633430

$-0.5799430$

$-0.5042320$

0.2572450

$-1.3436280$

1.2846790

1.4825610

0.4259970

$-0.7628960$

$-0.7122340$

$-1.1038980$

$-1.5638160$

$-1.6413470$

$-1.2564960$

0.3094050

0.6817010

1.1886420

1.3290020

0.9618670

1.6177010

$-0.3712600$

$-0.3894890$

$-0.1767240$

$-0.2228750$

$-1.5397000$

2.2425110

1.7803910

$-1.3859690$

0.1061290

1.9542900

2.3109960

$-0.3721940$

$-1.0531670$

$-1.8679980$

$-2.0199560$ 


\begin{tabular}{|c|c|c|c|c|c|c|c|}
\hline \multicolumn{2}{|c|}{54} & \multicolumn{2}{|l|}{$\mathrm{H}$} & 4.4681470 & \multicolumn{3}{|c|}{-2.3017830} \\
\hline & 55 & \multicolumn{2}{|l|}{$\mathrm{H}$} & \multicolumn{2}{|l|}{5.9221660} & \multicolumn{2}{|l|}{1.7403040} \\
\hline & 56 & \multicolumn{2}{|l|}{$\mathrm{H}$} & \multicolumn{2}{|l|}{8.2497540} & \multicolumn{2}{|l|}{1.1698080} \\
\hline & 57 & \multicolumn{2}{|l|}{$\mathrm{H}$} & \multicolumn{2}{|l|}{8.7726940} & \multicolumn{2}{|l|}{-1.0906530} \\
\hline & 58 & \multicolumn{2}{|l|}{$\mathrm{H}$} & \multicolumn{2}{|l|}{6.9331510} & \multicolumn{2}{|l|}{-2.7461360} \\
\hline & 59 & \multicolumn{2}{|l|}{$\mathrm{H}$} & \multicolumn{2}{|l|}{4.6307350} & \multicolumn{2}{|l|}{-2.1599070} \\
\hline & 60 & \multicolumn{2}{|l|}{$\mathrm{H}$} & \multicolumn{2}{|l|}{-2.4218270} & \multicolumn{2}{|l|}{-2.7438350} \\
\hline & 61 & \multicolumn{2}{|l|}{$\mathrm{H}$} & \multicolumn{2}{|l|}{-3.5016800} & \multicolumn{2}{|l|}{-2.2399410} \\
\hline & 62 & \multicolumn{2}{|l|}{$\mathrm{H}$} & 4.0973170 & & \multicolumn{2}{|l|}{-3.3125040} \\
\hline SCF Done: & \multicolumn{3}{|c|}{ E(RmPW1PW91) } & $=$ & \multicolumn{2}{|c|}{-1872.28012956} & A.U. \\
\hline \multicolumn{4}{|c|}{ Zero-point correction } & $=$ & \multicolumn{3}{|c|}{0.492147 (Hartree/Particle) } \\
\hline \multicolumn{4}{|c|}{ Thermal correction to Energy } & $=$ & \multicolumn{3}{|c|}{0.521518} \\
\hline \multicolumn{4}{|c|}{ Thermal correction to Enthalpy } & $=$ & \multicolumn{3}{|c|}{0.522462} \\
\hline Thermal ce & prrection $t$ & Gibbs Fre & Energy & $=$ & 0.4291 & & \\
\hline Sum of ele & ctronic an & zero-poin & Energies & $=$ & -1871. & 87982 & \\
\hline Sum of ele & ctronic an & $d$ thermal $\mathrm{E}$ & hergies & $=$ & -1871 & 58612 & \\
\hline Sum of ele & ctronic an & $d$ thermal $\mathrm{E}$ & thalpies & $=$ & -1871. & 57667 & \\
\hline Sum of ele & ctronic an & $d$ thermal $F$ & ee Energies & $=$ & -1871 & 50952 & \\
\hline Low freque & ncies --- & -3.7002 & -0.0008 & -0.0006 & 0.0009 & 1.3132 & 1.6231 \\
\hline Low frequ & encies --- & 9.5582 & 15.6618 & 23.9669 & & & \\
\hline
\end{tabular}

$-1.3536590$

$-0.0729180$

0.5740170

1.4771400

1.7417040

1.1036490

1.3758040

2.6951270

1.4057960

\section{References}

(S1) Ulrike, L.; Baumgartel, H. ELECTROCHEMICAL FORMATION AND CLEAVAGE OF BISIMIDAZOLYLS. J. Electroanal. Chem. 1977, 78, 133-143.

(S2) Pavitt, A. S.; Bylaska, E. J.; Tratnyek, P. G. Oxidation Potentials of Phenols and Anilines: Correlation Analysis of Electrochemical and Theoretical Values. Environ. Sci. Process. Impacts 2017, 19, 339-349.

(S3) Kistenmacher, A.; Baumgarten, M.; Enkelmann, V.; Pawlik, J.; Mullen, K. Novel Donor 1,4- 
Benzothiazino[2,3-b]Phenothiazine. J. Org. Chem. 1994, 59, 2743-2747.

(S4) Matsui, M.; Miyamoto, Y.; Shibata, K.; Takase, Y. Ozonization of Phenothiazine and Its Analogues. Bull. Chem. Soc. Jpn. 1984, 57, 2526-2530.

(S5) Pavlishchuk, V. V.; Addison, A. W. Conversion Constants for Redox Potentials Measured versus Different Reference Electrodes in Acetonitrile Solutions at $25^{\circ} \mathrm{C}$. Inorganica Chim. Acta 2000, 298, 97-102.

(S6) Frisch, M. J.; Trucks, G. W.; Schlegel, H. B.; Scuseria, G. E.; Robb, M. A.; Cheeseman, J. R.; Scalmani, G.; Barone, V.; Mennucci, B.; Petersson, G. A.; et al. Gaussian 09, 2009. 